ITLV/10972--177

UC-700

\title{
Preliminary Site
}

Characterization Report

Rulison Site, Colorado

August 1996

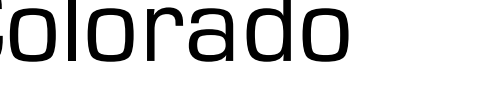


This report has been reproduced directly from the best available copy.

Available to DOE and DOE contractors from the Office of Scientific and Technical Information, P.O. Box 62, Oak Ridge, TN 37831; prices available from (423) 576-8401.

Available to the public from the National Technical Information Service, U.S. Department of Commerce, 5285 Port Royal Rd., Springfield, VA 22161, telephone (703) 487-4650. 


\title{
PRELIMINARY SITE CHARACTERIZATION REPORT RULISON SITE, COLORADO
}

\author{
IT CORPORATION \\ 2621 Losee Road, Building B-1, Suite 3050-01 \\ North Las Vegas, Nevada 89030
}

August 1996

Approved for public release; further dissemination unlimited.

This work performed for the U.S. Department of Energy by IT Corporation under Contract No.: DE-AC08-96NV10972 


\section{Abstract}

This report is a summary of environmental information gathered during a review of the documents pertaining to Project Rulison and interviews with personnel who worked on the project. Project Rulison was part of Operation Plowshare (a program designed to explore peaceful uses for nuclear devices). The project consisted of detonating a 43-kiloton nuclear device on September 10, 1969, in western Colorado to stimulate natural gas production. Following the detonation, a reentry well was drilled and several gas production tests were conducted. The reentry well was shut-in after the last gas production test and was held in standby condition until the general cleanup was undertaken in 1972. A final cleanup was conducted after the emplacement and testing wells were plugged in 1976. However, some surface radiologic contamination resulted from decontamination of the drilling equipment and fallout from the gas flaring during drilling operations. With the exception of the drilling effluent pond, all surface contamination at the Rulison Site was removed during the cleanup operations. All mudpits and other excavations were backfilled, and both upper and lower drilling pads were leveled and dressed.

This report provides information regarding known or suspected areas of contamination, previous cleanup activities, analytical results, a review of the regulatory status, the site's physical environment, and future recommendations for Project Rulison. Based on this research, several potential areas of contamination have been identified. These include the drilling effluent pond and mudpits used during drilling operations. In addition, contamination could migrate in the gas horizon.

The drilling effluent pond at the Rulison Site was used to store nonradioactive drilling mud during the drilling of the emplacement hole for the nuclear device. In 1994 and 1995, three pondsediment sampling events were conducted to evaluate the nature of this residual drilling fluid. The sampling indicated the presence of up to seven percent, by weight, of diesel fuel and the presence of chromium. The diesel fuel contained total petroleum hydrocarbon compounds in addition to benzene, toluene, ethylbenzene, and xylene. Prior to the detonation of the nuclear device, the sumps remaining from drilling the emplacement hole (with the exception of the drilling effluent pond previously mentioned) were cleaned and filled with earth.

Two natural gas production wells are located within 5 kilometers ( 3 miles) of the Rulison Site. Both wells are currently shut-in because current (1995) low gas prices make production uneconomical. If contamination enters the gas horizons, it should appear in the water or gas from 
one or both of these wells. Tritium is the most likely contaminant to be found in the natural gas or groundwater from the production wells because it is the most mobile of the radionuclides produced by detonation of the nuclear device.

Based on information provided in this report, the following tasks should be completed to close the remaining information gaps for Project Rulison:

- Complete the human health baseline risk assessment

- Collect gas/water samples from the gas wells closest to the shot cavity

- Characterize the mudpit located by the reentry (RE-X) well

- Continue the Long-Term Hydrologic Monitoring Program

- Develop action plan in the event contamination is found. 


\section{Table of Contents}

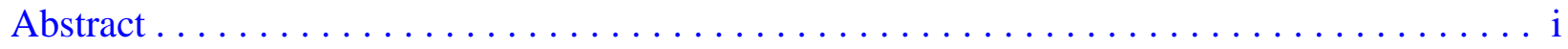

List of Figures $\ldots \ldots \ldots \ldots \ldots \ldots \ldots \ldots \ldots \ldots \ldots \ldots \ldots \ldots \ldots \ldots \ldots \ldots$

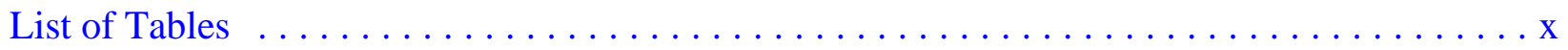

List of Acronyms and Abbreviations $\ldots \ldots \ldots \ldots \ldots \ldots \ldots \ldots \ldots \ldots \ldots \ldots \ldots \ldots$

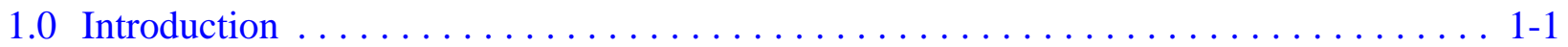

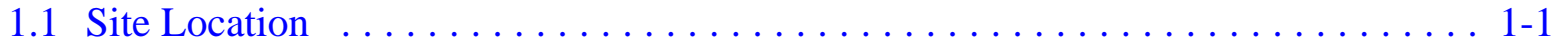

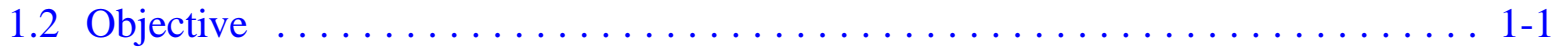

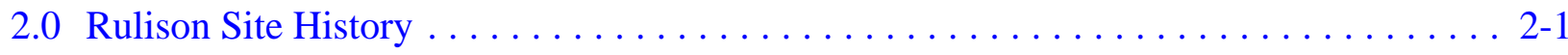

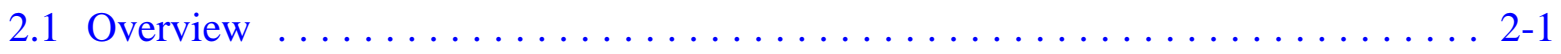

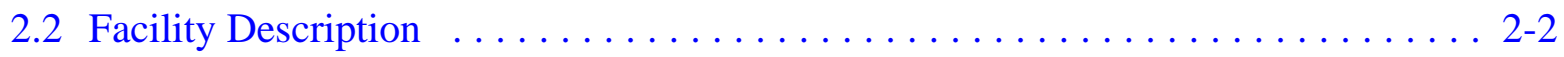

2.2.1 Known or Suspected Areas of Contamination . . . . . . . . . . . . 2-2

2.2.1.1 Drilling Effluent Pond . . . . . . . . . . . . . . 2-2

2.2.1.2 Mudpits ......................... 2-2

2.2.1.3 Natural Gas Wells . . . . . . . . . . . . . . . . . . 2-4

2.2.2 Previous Cleanup . . . . . . . . . . . . . . . . . . . . . . .

2.2.2.1 Initial Cleanup Effort (July 10 through 25, 1972) . . . . . . . . 2-6

2.2.2.2 Final Cleanup Effort (September 1 through October 12, 1976) . . 2-10

2.2.2.3 Plugging and Abandonment Operations . . . . . . . . . . 2-11

2.2.2.3.1 R-EX Well ..................... 2-11

2.2.2.3.2 R-E Well ... . . . . . . . . . . . . . 2-13

2.2.2.4 Environmental Sampling and Survey Programs . . . . . . . . . 2-13

2.2.2.4.1 First Sampling Program . . . . . . . . . . . . . 2-15

2.2.2.4.2 Second Sampling Program . . . . . . . . . . . . . 2-15

2.2.2.4.3 Third Sampling Program . . . . . . . . . . . 2-35

2.2.2.4.4 Aerial Radiological Survey . . . . . . . . . . . . . 2-55

2.2.2.5 Sampling Summary . . . . . . . . . . . . . . . 2-55 


\section{Table of Contents (Continued)}

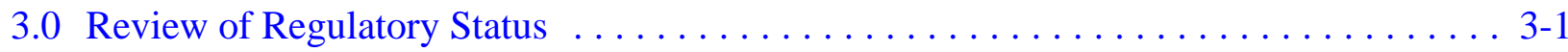

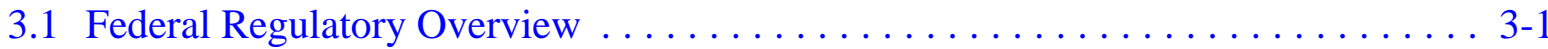

3.2 State Regulatory Overview $\ldots \ldots \ldots \ldots \ldots \ldots \ldots \ldots \ldots \ldots \ldots \ldots \ldots . .3$

3.2.1 Property of Historic, Archaeological, or Architectural Significance ... . . 3-3

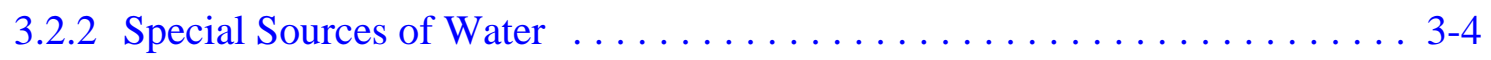

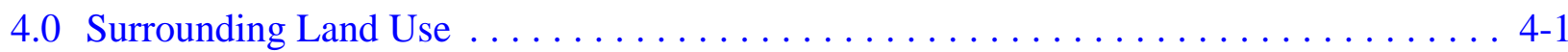

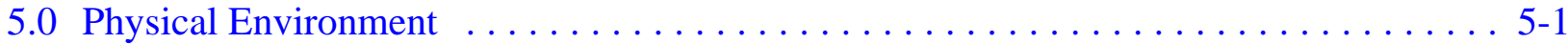

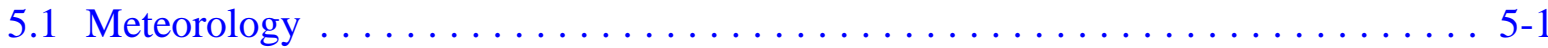

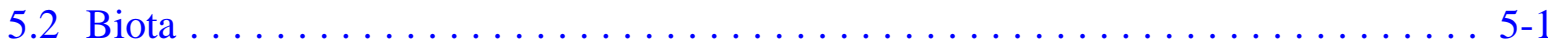

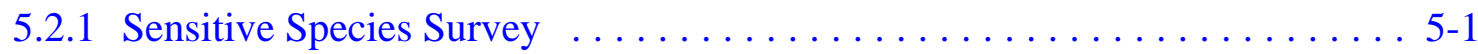

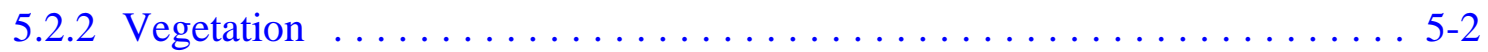

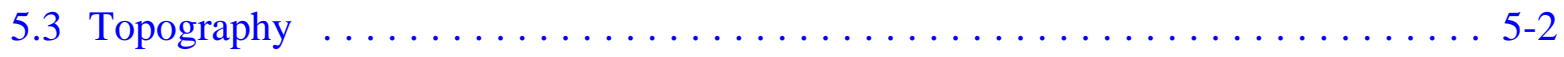

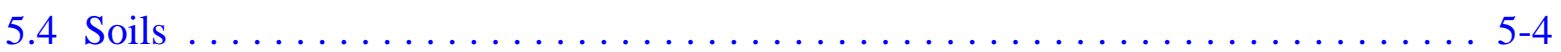

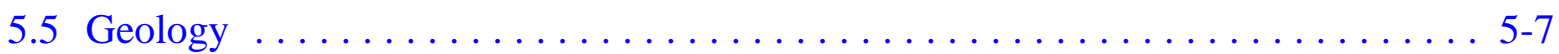

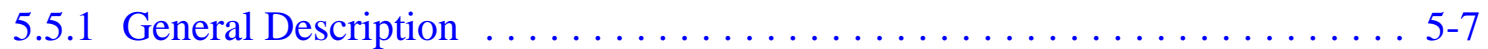

5.5 .2 Surficial Geology $\ldots \ldots \ldots \ldots \ldots \ldots \ldots \ldots \ldots \ldots \ldots \ldots \ldots \ldots \ldots \ldots$

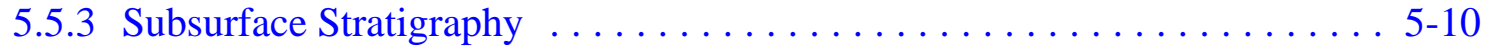

5.5.3.1 Mancos Shale .............................. 5-10

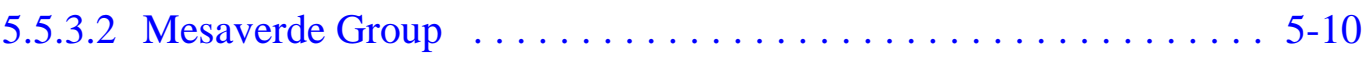

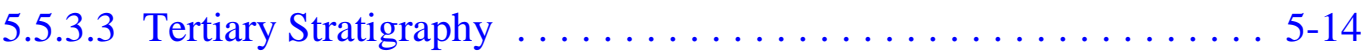

5.5.4 Natural Gas Production in the Rulison Area .................. 5-15

5.5.5 Gas Reservoir Characteristics . . . . . . . . . . . . . . . . . 5-16

5.6 Surface Water . . . . . . . . . . . . . . . . . . . . . . . . . . 5-16

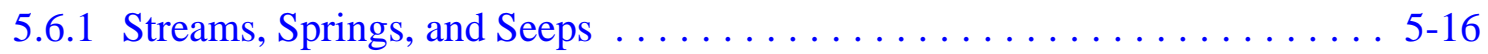

5.6 .2 Wetlands . . . . . . . . . . . . . . . . . . . . . . . . . . 5

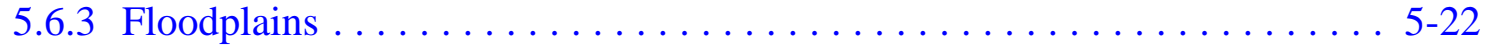

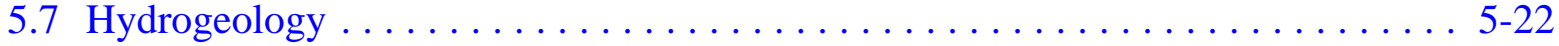

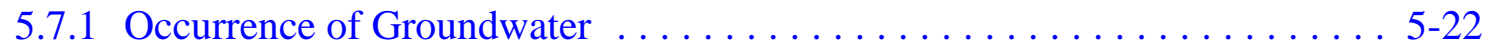

5.7.1.1 Hydraulic Characteristics ...................... 5-25

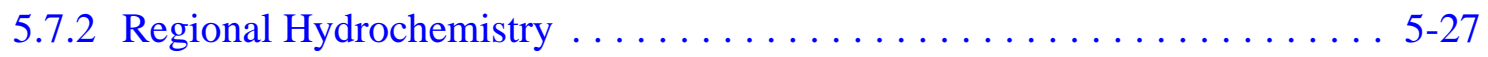

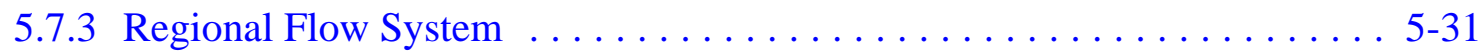




\section{Table of Contents (Continued)}

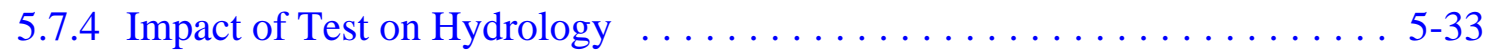

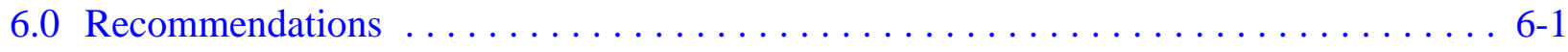

6.1 Complete Human Health Risk Assessment . . . . . . . . . . . . . . . . . . . 6 6-1

6.2 Collect Gas/Water Samples . . . . . . . . . . . . . . . . . . . . 6-1

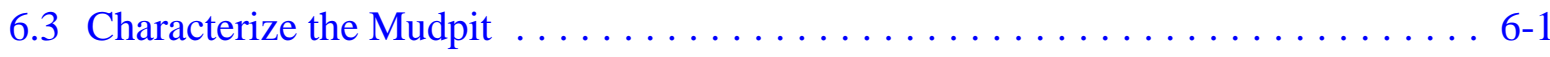

6.4 Continue the Long-Term Hydrologic Monitoring Program $\ldots \ldots \ldots \ldots \ldots .6-1$

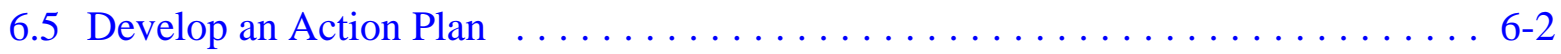

7.0 References $\ldots \ldots \ldots \ldots \ldots \ldots \ldots \ldots \ldots \ldots \ldots \ldots \ldots \ldots \ldots \ldots \ldots \ldots \ldots, 7-1$

Appendix A - Project Rulison, Long-Term Hydrologic Monitoring Program Analytical Results, 1972-1994, Garfield County, Colorado . . . . . . . . . . . . . A-1

Appendix B - Records of Selected Wells and Springs, Rulison Project Area . . . . . . . . . B-1 Appendix C - Chemical Analyses of Groundwater and Surface Water, Project Rulison Area .................................. C-1

Appendix D - Radiochemical Analyses of Spring, Well, and Stream Waters, Rulison

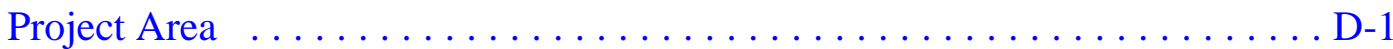




\section{List of Figures}

Number

Title

Page

1-1 Rulison Site Location Map, Garfield County, Colorado . . . . . . . . . . . . . 1-2

2-1 Rulison Site Plan Postshot Reentry, June 1, 1970, Garfield County, Colorado _ . . 2-3

2-2 Water Wells and Springs in the Vicinity of Project Rulison,

Garfield and Mesa Counties, Colorado . . . . . . . . . . . . . . . . . 2-5

2-3 Rulison Site at Completion of Flare Testing, April 1971,

Garfield County, Colorado . . . . . . . . . . . . . . . . . . . 2-7

2-4 Rulison Site at Completion of the General Site Cleanup Effort, July 1972, Garfield County, Colorado . . . . . . . . . . . . . . . . . . . 2-9

2-5 Project Rulison Exploratory - Reentry Well (R-EX) “As Built” Plugging Condition, July 1972, Garfield County, Colorado . . . . . . . . . . . . . 2-12

2-6 Project Rulison, Emplacement Hole (R-E) “As Built” Plugging Condition, July 1972, Garfield County, Colorado . . . . . . . . . . . . . . . . . . . . 2-14

2-7 Project Rulison Soil Sample Locations Taken after Completion of Production Testing, April 1971, Garfield County, Colorado . . . . . . . . . . . 2-16

2-8 Project Rulison Soil Sample Locations Taken after Completion of Production Testing, April 1971, Garfield County, Colorado

2-9 Project Rulison Soil Sample Locations Taken at Time of Site Cleanup, July 1972, Garfield County, Colorado . . . . . . . . . . . . . . . . . 2-18

2-10 Project Rulison Soil Sample Locations Taken at Time of Site Cleanup, July 1972, Garfield County, Colorado 


\section{List of Figures (Continued)}

Number

Title

Page

2-11 Project Rulison, Long-Term Hydrologic Monitoring Program

Sampling Data Points, December 1971, Garfield County, Colorado ........ 2-36

2-12 Soil Sampling, Rulison Tank Area, October 1976, Garfield County, Colorado . . 2-38

2-13 Soil Sampling, Rulison Separator Pan Area (previous pipe spill),

September 30, 1976, Garfield County, Colorado .................. 2-39

2-14 Surface Soil Sampling, Rulison Separator Spill, September 1, 1976,

Garfield County, Colorado ............................ 2-40

2-15 Surface Soil Sampling, Rulison Separator Spill Survey after 15 Drum Soil

Removal, September 16, 1976, Garfield County, Colorado .............. 2-41

2-16 Soil Sampling, Rulison Separator Spill after Removal of Five Additional

Drums of Soil, September 21, 1976, Garfield County, Colorado . . . . . . . . . 2-42

2-17 Soil Sampling, Rulison Separator Spill, September 23, 1976,

Garfield County, Colorado .......................... 2-46

2-18 Rulison Separator Spill (Trenches), October 1, 1976,

Garfield County, Colorado ........................... 2-47

2-19 Soil Sampling, Rulison R-EX Decon Pan Area, October 7, 1976,

Garfield County, Colorado ............................. 2-48

2-20 Soil Sampling, Rulison R-E Wellhead Cellar Area, October 7, 1976,

Garfield County, Colorado ............................. 2-49 


\section{List of Figures (Continued)}

Number

3-1 Soil Sampling, Potential Hazardous Waste Release Sites

at the Rulison Test Site, September 1976, Garfield County, Colorado . . . . . . . 3-2

4-1 Project Rulison, Garfield County, Land Ownership Map . . . . . . . . . . . . . . 4-2

5-1 Topographic Map of Garfield County, Colorado, Project Rulison . . . . . . . . . . 5-5

5-2 U.S. Department of Agriculture Soil Conservation Service Soils Map,

Rifle Area, Colorado, Rulison Project . . . . . . . . . . . . . . . . . . 5-6

5-3 Rulison Site, Piceance Creek Basin - Regional Map and Structural

Interpretation, Garfield and Mesa Counties, Colorado $\ldots \ldots \ldots \ldots \ldots$. . . . . .

5-4 Rulison Site Piceance Creek Basin Schematic Cross Section,

Garfield and Mesa Counties, Colorado . . . . . . . . . . . . . . . . 5-9

5-5 Project Rulison Generalized Geologic Cross Section,

Garfield County, Colorado . . . . . . . . . . . . . . . . . . 5-11

5-6 Rulison Site Stratigraphic Column, Garfield County, Colorado . . . . . . . . . . 5-12

5-7 Correlation of Paleoenvironmental Depositional Units at the Multi-Well Experiment Site with Regional Stratigraphic Nomenclature,

Garfield County, Colorado . . . . . . . . . . . . . . . . . . . . . . 5 5-13

5-8 Structure Contour Map of the Top of Marine Intervals

(Rollins-Trout Creek) of the Piceance Basin, Colorado . . . . . . . . . . . . . 5-15

5-9 Compilation Map of the Fracture Orientation, Piceance Basin, Colorado . . . . 5-17 


\section{List of Figures (Continued)}

Number

5-10 General Site Layout Diagram, Rulison Drilling Effluent Pond, January 1995 . . . 5-19

5-11 Project Rulison Emplacement and Reentry Well Configuration . . . . . . . . . . . 5-24

5-12 Location of the U.S. Geological Survey Water-Sampling Points, Project Rulison . . . . . . . . . . . . . . . . . . . . . . . . . . . 5-28

5-13 Piceance Creek Basin, Rio Blanco, Garfield, and Mesa Counties, Colorado . . . 5-32 


\section{List of Tables}

Number

Title

Page

2-1 Tritium in Rulison Soil Moisture Postproduction Test - April 23, 1971 . . . . . . 2-20

2-2 Tritium in Rulison Soil Moisture - July $1972 \ldots \ldots \ldots \ldots$. . . . . . . . . 25

2-3 Tritium in Vegetation - July $1972 \ldots \ldots \ldots \ldots \ldots$. . . . . . . . . . . . . . . . . .

2-4 Tritium in Soil at Sampling Point N-14.2, E-.7 - October $1976 \ldots \ldots$. . . . . . 2-43

2-5 Tritium in Soil at Sampling Point S-24.6, E-13.7 - October 1976 . . . . . . . . 2-44

2-6 Tritium in Soil at Spill Area after Decontamination - October 1976 . . . . . . . 2-50

2-7 Tritium in Decontamination Work Area Soil . . . . . . . . . . . . . . . . 2-52

2-8 Tritium in Soil at R-E Wellhead - October $1976 \ldots \ldots \ldots \ldots$. . . . . . . . . . . 2-54

5-1 List of Reptile and Amphibian Species Observed during the

Sensitive Species Survey of the Rulison Site, Colorado, June 1993 . . . . . . . . . . 5-2

5-2 List of Bird Species Observed during the Sensitive

Species Survey of the Rulison Site, Colorado, June 1993 . . . . . . . . . . . . 5 5-3

5-3 List of Mammal Species Observed during the Sensitive

Species Survey of the Rulison Site, Colorado, June 1993 . . . . . . . . . . . . 5-4

5-4 List of Dominant Plant Species - Rulison Site Wetland Survey

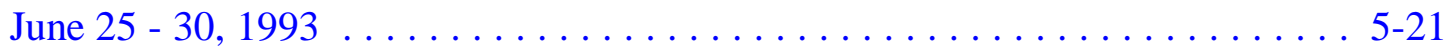

5-5 Summary of Hydraulic Tests, Hole R-EX . . . . . . . . . . . . . . 5-26

5-6 Summary of the Water-Bearing Characteristics of the Geologic Formation . . . . 5-29 


\section{List of Tables (Continued)}

Number

Title

Page

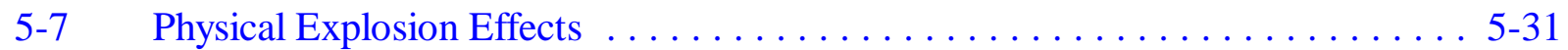

5-8 R-EX Drill Stem Test Formation Pressures . . . . . . . . . . . . . . . . . . . . 5-34

5-9 Distribution Coefficients of Strontium-85 and Cesium-137 for

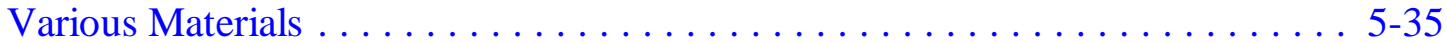




\section{List of Acronyms and Abbreviations}

\begin{tabular}{|c|c|}
\hline $\mathrm{AEC}$ & U.S. Atomic Energy Commission \\
\hline amsl & Above mean sea level \\
\hline BLM & U.S. Bureau of Land Management \\
\hline BMI & Battelle Memorial Institute \\
\hline${ }^{\circ} \mathrm{C}$ & Degree(s) Celsius \\
\hline CDNR & State of Colorado Department of National Resources \\
\hline CERCLA & Comprehensive Environmental Response, Compensation, and Liability Act \\
\hline CFR & Code of Federal Regulations \\
\hline CG & Contaminant guideline \\
\hline $\mathrm{Ci}$ & Curie(s) \\
\hline $\mathrm{cm}$ & Centimeter(s) \\
\hline $\mathrm{cm}^{2}$ & Square centimeter(s) \\
\hline $\mathrm{cm}^{3}$ & Cubic centimeter(s) \\
\hline DOE & U.S. Department of Energy \\
\hline $\mathrm{DOE} / \mathrm{NV}$ & DOE/Nevada Operations Office \\
\hline dpm & Disintegration(s) per minute \\
\hline DRI & Desert Research Institute \\
\hline ERDA & U.S. Energy Research and Development Administration \\
\hline EPA & Environmental Protection Agency \\
\hline${ }^{\circ} \mathrm{F}$ & Degree(s) Fahrenheit \\
\hline \multicolumn{2}{|c|}{ FEMA Federal Emergency Management Agency } \\
\hline FIRM & Flood Insurance Rate Map \\
\hline $\mathrm{ft}$ & Foot (feet) \\
\hline gpd & Gallon(s) per day \\
\hline HRS & Hazard Ranking System \\
\hline in. & $\operatorname{Inch}(\mathrm{es})$ \\
\hline IT & IT Corporation \\
\hline $\mathrm{K}_{\mathrm{d}}$ & Distribution coefficient \\
\hline $\mathrm{km}$ & Kilometer(s) \\
\hline LTHMP & Long-Term Hydrologic Monitoring Program \\
\hline$\mu \mathrm{d}$ & Microdarcy(ies) \\
\hline $\mathrm{m}$ & Meter(s) \\
\hline$m^{2}$ & Square meter(s) \\
\hline
\end{tabular}




\section{List of Acronyms and Abbreviations (Continued)}

$\begin{array}{ll}\mathrm{m}^{3} & \text { Cubic meter(s) } \\ \mu \mathrm{Ci} / \mathrm{mL} & \text { MicroCurie(s) per milliliter } \\ \mathrm{MCF} & \text { Million cubic feet } \\ \mathrm{mi} & \text { Mile(s) } \\ \mathrm{mg} & \text { Milligram(s) } \\ \mathrm{mL} & \text { Milliliter(s) } \\ \mathrm{mrad} & \text { Millirad(s) } \\ \text { NEPA } & \text { National Environmental Policy Act } \\ \mathrm{pCi} / \mathrm{mL} & \text { PicoCurie(s) per milliliter } \\ \mathrm{pCi} / \mathrm{g} & \text { PicoCurie(s) per gram } \\ \mathrm{pCi} / \mathrm{L} & \text { PicoCurie(s) per liter } \\ \mu \mathrm{R} / \mathrm{h} & \text { Microroentgen(s) per hour } \\ \text { R-E } & \text { Emplacement well } \\ \text { R-EX } & \text { Reentry well } \\ \text { SGZ } & \text { Surface ground zero } \\ \text { SCS } & \text { Soil Conservation Service } \\ \text { USDA } & \text { U.S. Department of Agriculture } \\ \text { USGS } & \text { U.S. Geological Survey } \\ \text { USPHS } & \text { U.S. Public Health Service } \\ & \end{array}$




\subsection{Introduction}

\subsection{Site Location}

The Rulison Site is located in Section 25, Township 7 South, Range 95 West (6th Principal Meridian), Garfield County, Colorado, approximately 19 kilometers (km) (12 miles [mi]) southwest of Rifle, Colorado, and approximately $65 \mathrm{~km}$ (40 mi) northeast of Grand Junction, Colorado (Figure 1-1). The site can be accessed by traveling west on I-70 from Rifle, $22 \mathrm{~km}$ (14 mi) to the town of Parachute. Then proceeding south from Parachute, up the Battlement Creek Valley, approximately $13 \mathrm{~km}$ (8 mi) to surface ground zero (SGZ).

\subsection{Objective}

The objective of this preliminary site characterization report is to summarize the information gathered during the recent literature search and interview process. The documents that have been reviewed were gathered from the U.S. Department of Energy (DOE) resource centers and Central Files and ranged from field personnel daily logs to issued reports dated from the projects' origination to current data from field activities. The personnel who were interviewed included local residents and retired or current DOE and contractor employees who were present during the testing. Information gathered from these sources has been evaluated to provide a clear picture of the site, including physical characteristics, testing, cleanups, and potential contaminated areas. This preliminary site characterization report will be used to identify potential DOE liabilities, formulate baseline risk assessments, and develop field work plans which will be implemented during the Phase II-Field Site Characterization process. 


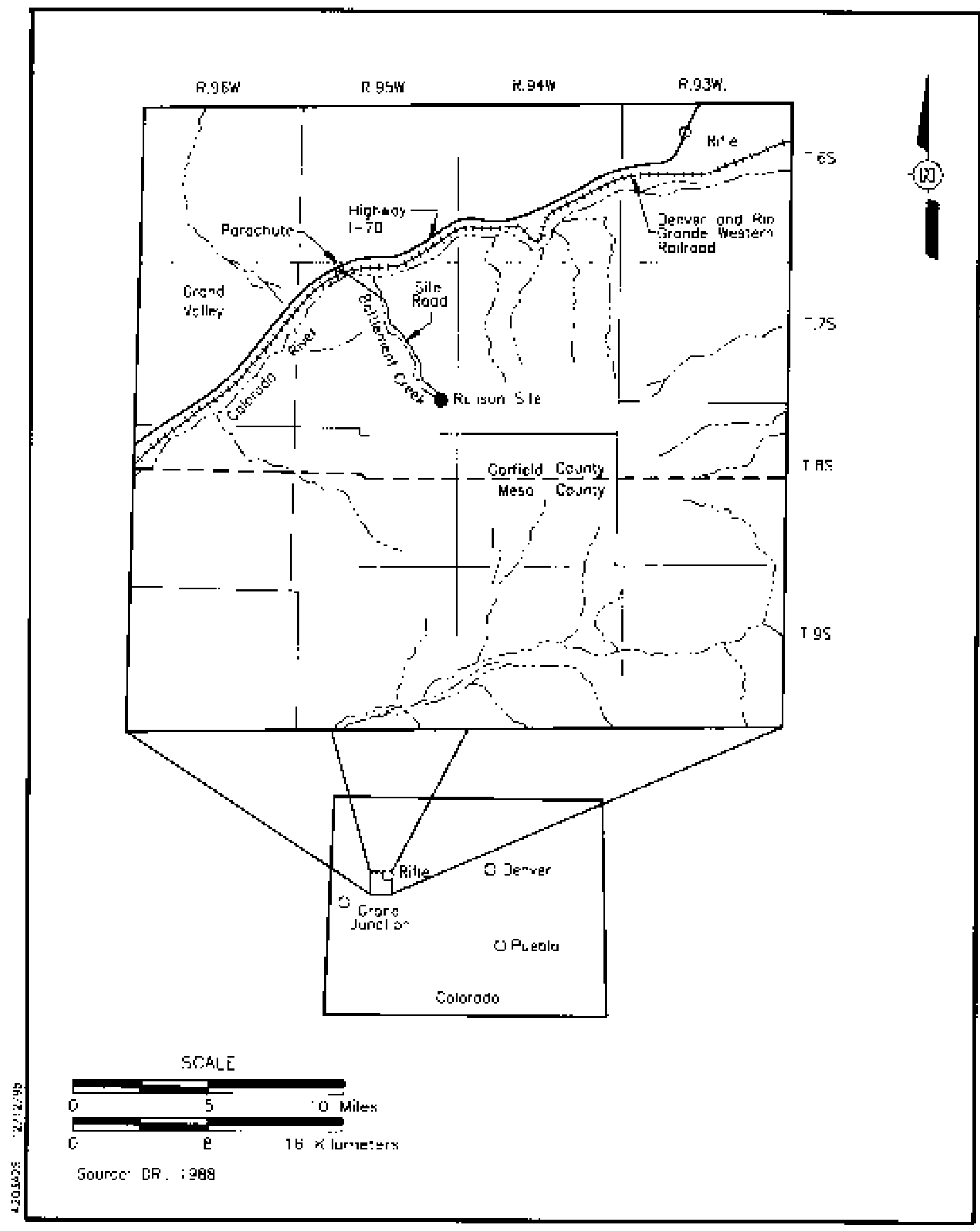

Figure 1-1

Ruligon Site Location Map, Garfleld County, Colorado 


\subsection{Rulison Site History}

\subsection{Overview}

The Rulison Project was the second of three joint government/industry, gas-production stimulation experiments conducted under the Plowshare Program, a project designed to develop peaceful uses of nuclear explosions. Project Rulison was a joint project between the U.S. Atomic Energy Commission (AEC) (currently known as the U.S. Department of Energy) and the Austral Oil Company. Under this program, the feasibility of stimulating natural gas production in lowpermeability, gas-producing geologic formations with underground nuclear explosions was studied. On September 10, 1969, a 43-kiloton nuclear device was detonated at a depth of 2,568 meters $(\mathrm{m})(8,426$ feet [ft]) below the ground surface. Redrilling of the former pre-shot exploratory hole which was then converted into the reentry well (R-EX), designed for conducting production testing of the stimulated zone, was located $300 \mathrm{ft}$ southeast of the emplacement well (R-E) and was completed in October 1970.

Production testing and data evaluation took place over a seven month period between October 1970 and April 1971, and included four separate flow periods. Approximately 12.0 million stock cubic m (455 million stock cubic $\mathrm{ft}$ ) of natural gas were produced. The well was shut-in after the last test and left in a standby condition until a general cleanup was undertaken in 1972. Cleanup activities were conducted at the site from July 10 through July 25, 1972, to remove all extraneous materials and equipment not required for gas production. A final cleanup was conducted after the emplacement and testing wells were plugged in 1976. Neither the Austral Oil Company nor the U.S. Energy Research and Development Administration (ERDA) developed any plans to commercially produce the available natural gas. Accordingly, during the period of September 1 , 1976, through October 12, 1976, the R-E and R-EX wells were plugged and abandoned, and the equipment that remained after the 1972 general cleanup was decontaminated as necessary and removed from the site (Eberline, 1977, p. 2). Some surface radiologic contamination resulted from decontamination of drilling equipment and fallout from the gas flaring (DRI, 1988, p. 3.6.18); however, except for the drilling effluent pond, all surface contamination was removed during site clean-up operations. 


\subsection{Facility Description}

\subsubsection{Known or Suspected Areas of Contamination}

Based on the review of available documentation and field sampling activities, several known or suspected areas of subsurface contamination are present. These include the drilling effluent pond and the mudpits used during drilling operations. In addition, contamination may be present in natural gas or water produced from nearby gas wells. Each of these locations is discussed below.

\subsubsection{Drilling Effluent Pond}

The effluent pond at the Rulison Site was used to store nonradioactive drilling mud during the boring of the emplacement hole for the nuclear device (Well R-E). The drilling fluids consisted of a bentonitic drilling mud with additives (such as diesel fuel and chrome lignosulfonate) to improve drilling characteristics. Most of the drilling mud was removed from the pond when the site was cleaned up and decommissioned in 1972; however, some residual fluid was left in the pond. In 1994 and 1995, three pond-sediment sampling events were conducted to evaluate the nature of this residual drilling fluid. The sampling indicated the presence of up to 7 percent, by weight, of diesel fuel as well as the presence of chromium. The diesel fuel contains total petroleum hydrocarbon compounds in addition to benzene, toluene, ethylbenzene, and xylene.

The DOE/Nevada Operations Office (DOE/NV) Nevada Environmental Restoration Project has undertaken a voluntary removal action to clean up the contaminated pond sediments, following which the pond will be restored to support an aquatic ecosystem. It is expected that pond restoration will be completed during the summer of 1996.

\subsubsection{Mudpits}

A pre-shot bioenvironmental survey of the area around the Rulison Site was made early in 1969 by Battelle Memorial Institute (BMI) (AEC, 1973b, p. 50). The objectives were to characterize the ecological setting of the project site and to identify any potential adverse consequences, as a result of prior project activities, which might require preventive or remedial action.

The only significant bioenvironmental hazard identified during the pre-shot survey was the possible danger of pollution of Battlement Creek by drilling wastes or other contaminants resulting from drilling operations. Sump ponds used in drilling the R-EX and the R-E wells were located very close to the channel of the East Fork of Battlement Creek (Figure 2-1). 


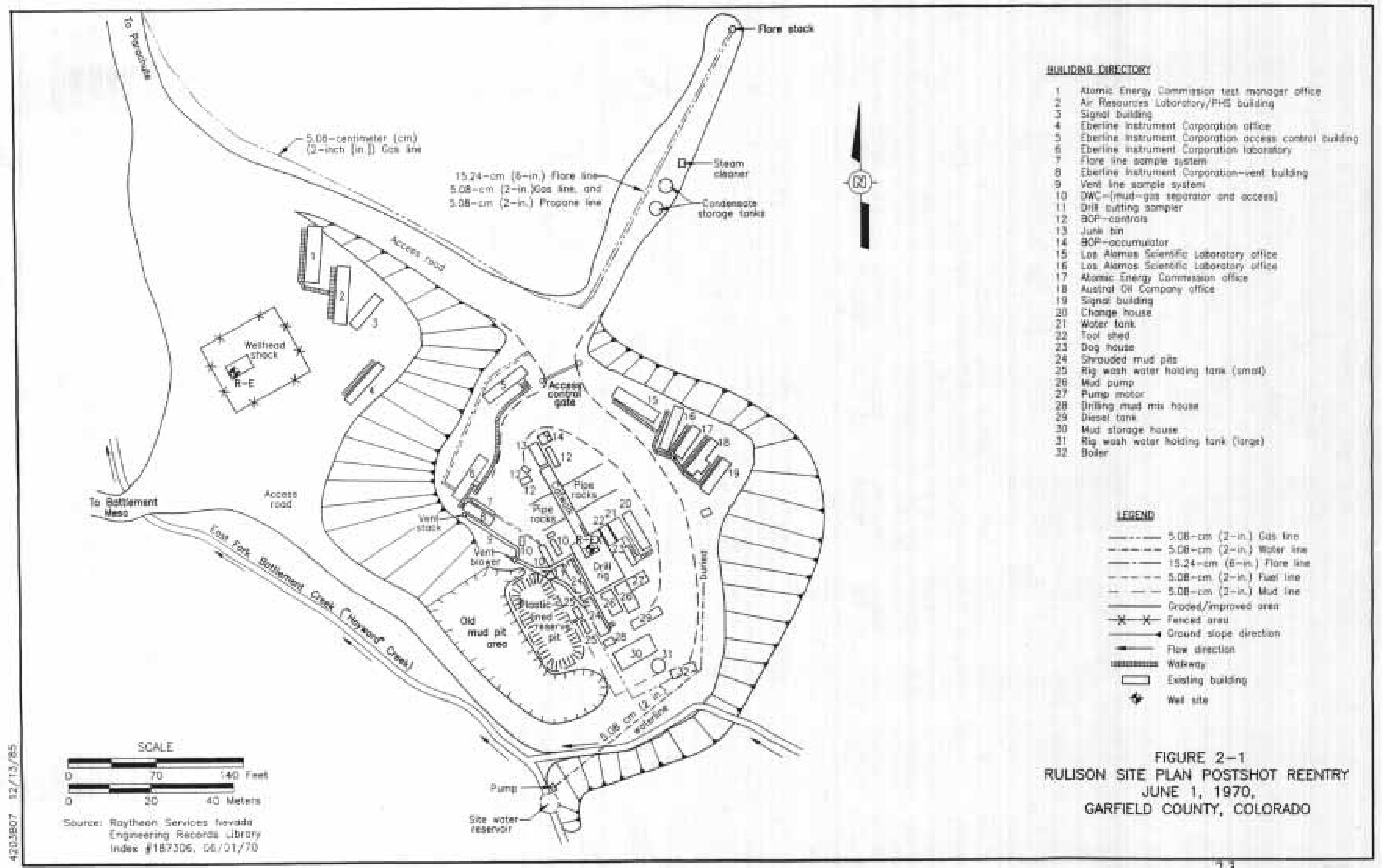


A sump failure occurred during the drilling of the pre-shot exploratory hole (Well R-EX) in December 1967, which killed fish in the stream below the site and temporarily contaminated the domestic and stock water supplies of some of the Morrisania Mesa residents. The pre-shot bioenvironmental survey report recommended that adequate precautions be taken to prevent any further pollution of the Battlement Creek watershed during the final site preparation and detonation phase. A water sampling plan for evaluating the effectiveness of these precautionary measures was also outlined. Results of a pre- and postshot stream water sampling program carried out by the Colorado Department of Health (Appendices B, C, and D) indicated these precautions were successful. In addition, springs and wells in the vicinity of the Rulison Site were sampled by the U.S. Geological Survey (USGS) (Figure 2-2) both before and after the detonation. While an increase in flow from springs and flow in Battlement Creek was observed immediately following the shot, the flow in all cases returned to pre-shot levels within a short time.

During a visit to the site between June 15 and 17, 1970, BMI and AEC personnel reported that oil and water had been running into Battlement Creek from one of the old mud sumps located next to the creek (Mason, 1970). Close examination of this area showed that most of the water was from snow buried at the time the mudpit was constructed. Two samples of water coming from the old sump were taken and submitted to the U.S. Public Health Service (USPHS) for analysis, and both samples contained elevated levels of hydrocarbons. Although the levels of hydrocarbons were an order of magnitude higher than the USPHS Drinking Water Standards, this was not considered a problem at the time because of the dilution factor when the stream flowed into Battlement Creek.

Prior to the detonation, the sumps remaining from drilling of the emplacement hole, with the exception of the drilling effluent pond previously mentioned, were cleaned and filled with earth (AEC, 1973b, p. 50). During site decommissioning in 1976, all mudpits and other excavations were backfilled and both the upper and lower drilling pads were leveled and dressed (ERDA, 1977, p. 5).

\subsubsection{Natural Gas Wells}

Two natural gas production wells are located near the Rulison Site (Section 5.5.4). These wells are the Federal 28-95, located $4.3 \mathrm{~km}(2.7 \mathrm{mi})$ west and the Federal 14-95 located $4.3 \mathrm{~km}$ (2.7 mi) to the northwest of SGZ (Figure 2-2). These wells have changed possession several 


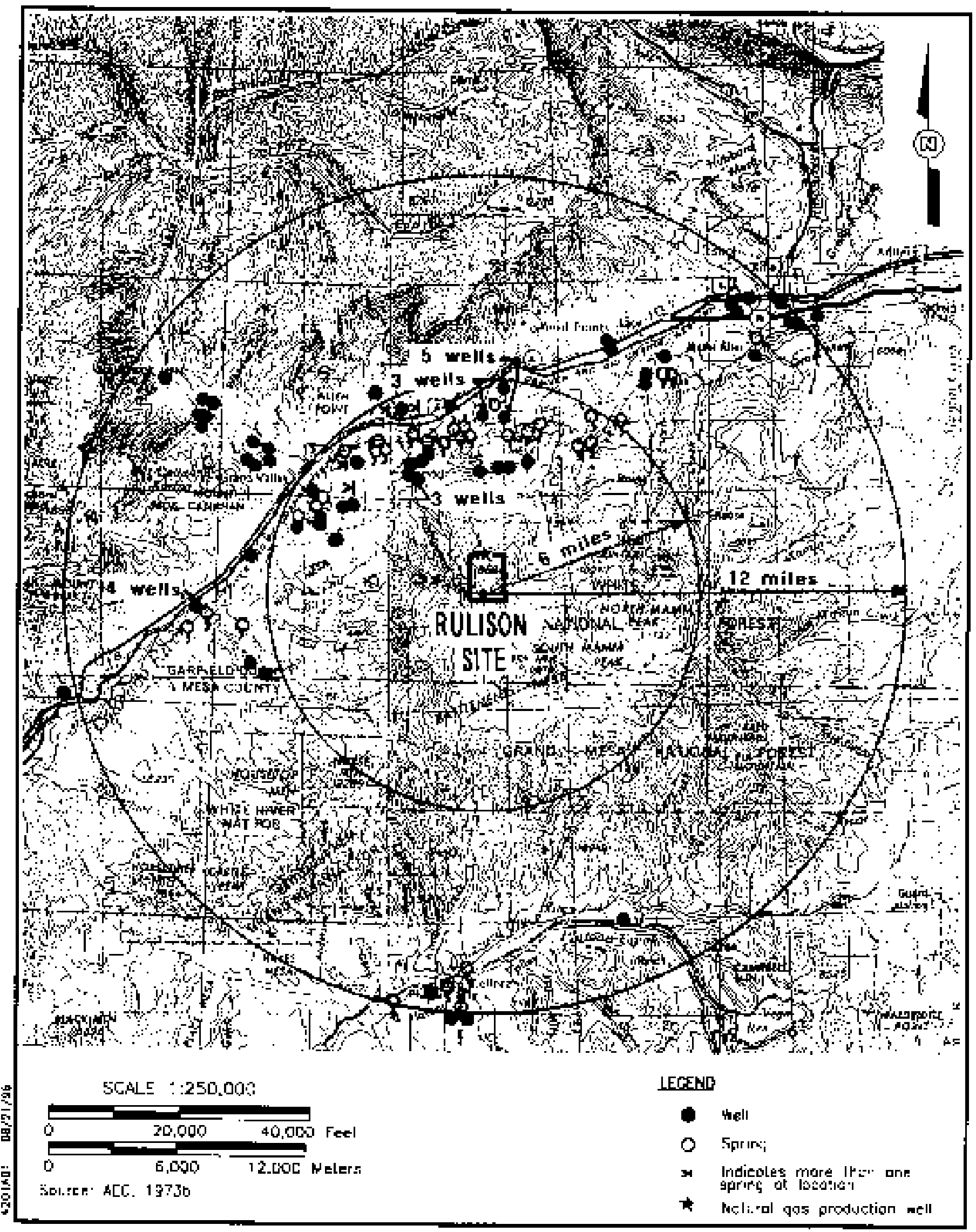

Figure 2-2

Water Wells and Springs in the Vicinity of Project Rulison, Garfield and Mega Counlies, Colorado 
times since they were initially completed and have produced gas and minor amounts of water on an intermittent basis. Both wells are currently shut-in because of low gas prices.

If any contamination enters the gas horizons, it would be expected to appear in the water or gas from these wells. Since natural gas production from the Mesaverde Group is primarily from fractures, and the dominant fracture strike in the Rulison area is northwest-southeast, then contaminated natural gas or groundwater may be drawn by well production activity or by natural gradients toward these production wells, principally the Federal 14-95. The highly anisotropic nature of the fracturing in the Mesaverde limits the potential for contaminated gas or groundwater production from wells that are or may be located in other directions from the Rulison Site.

Tritium is the contaminant most likely to be found in the natural gas or groundwater from the production wells because it is the most mobile of the radionuclides produced by the nuclear device. The amount of tritium and other radionuclides produced by the explosion of the nuclear device is still classified information.

\subsubsection{Previous Cleanup}

The decontamination effort at the Rulison Site was divided into two operations: the general (initial) cleanup in 1972 and the final cleanup in 1976 (Eberline, 1977). The total amount of tritium shipped from the Rulison Site as a result of both cleanup operations was estimated to be 0.781 curies $(\mathrm{Ci})$. No other radionuclides were reported in either cleanup, and no burial of radioactive solids occurred at the Rulison Site. All on-site equipment was removed during the final cleanup with the exceptions of the R-E wellhead, a power pole with a fuse box, a telephone line, a concrete slab, and a small monument over the emplacement well stating drilling restrictions at the site (DRI, 1988, p. 3.6.5).

\subsubsection{Initial Cleanup Effort (July 10 through 25, 1972)}

Prior to the initial cleanup, the site was in standby condition with all surface equipment intact (Figure 2-3). During this cleanup, all items of equipment and material that were not required for production testing were removed from the site. Following the cleanup, soil, water, and vegetation sampling was conducted which is further discussed in Section 2.2.2.4.2. A release log was maintained to describe each item and to record its radiological condition if it were to be released for unrestricted use. There were 504 uncontaminated and decontaminated items logged and released, and those items that could not be economically decontaminated were included in 


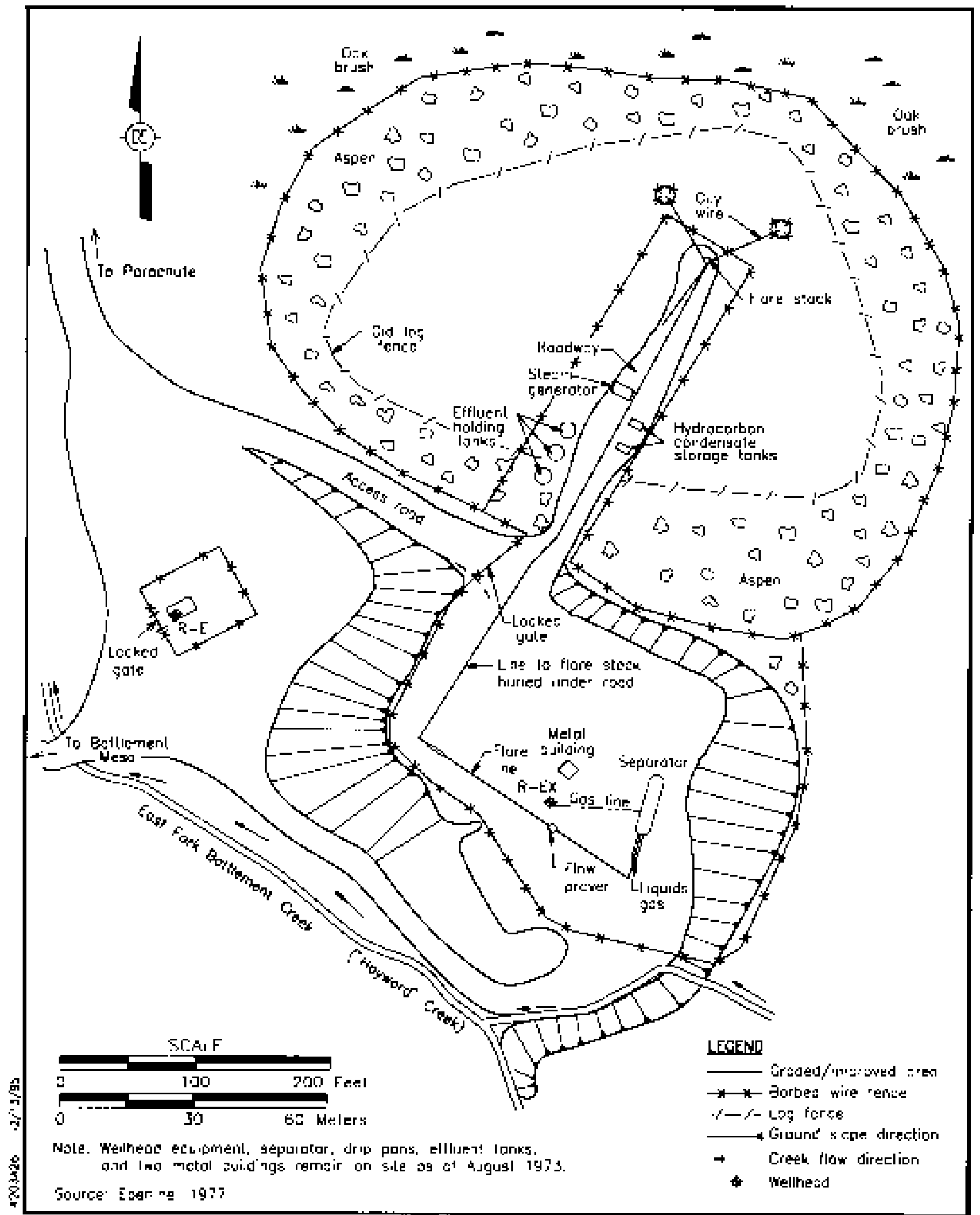

Figure 2-3

Rullson Site at Completion of Flare Testing,

April 1971, Gartield County, Colorado 
the material shipped to Beatty, Nevada, for burial at the Nuclear Engineering Company facility, now known as U.S. Ecology. Decontamination operations were conducted in a large, sheet-metal pan using saturated steam and Steamzall ${ }^{\circledR}$. The guideline limits for release of material were 1,000 counts per minute beta-gamma removable from any 100 square centimeters $\left(\mathrm{cm}^{2}\right)$, and a total of 0.4 millirad $(\mathrm{mrad}) /$ hour at 1 centimeter $(\mathrm{cm})$ from the surface, through not more than a 7 milligram (mg)/ $\mathrm{cm}^{2}$ absorber. In practice, the actual removable contamination for released items in each case was not above background ( $0.02 \mathrm{mrad} /$ hour $)$ of the site area. Items of equipment and materials found to be clean, or that had been decontaminated, were removed to the Austral storage yard at Rifle, Colorado. Items in this category included the flare stack and the sections of 2-inch (in.) and 6-in. pipe that ran between the north gate and the separator.

On July 20, 1972, 11.36 cubic meters $\left(\mathrm{m}^{3}\right)$ (3,000 gallons) of decontamination fluid containing $0.69 \mathrm{Ci}$ of tritium were shipped by tank truck to the waste facility at Beatty, Nevada. On July 22, 1972, thirty-two packages of contaminated solid waste and six 55-gallon steel drums of solidified liquid waste, both containing an estimated $0.073 \mathrm{Ci}$ of tritium, were also shipped.

Upon completion of the 1972 cleanup, the following equipment was left on site (Figure 2-4):

- The high-pressure wellhead and pressure measuring equipment and instruments at the $\mathrm{R}-\mathrm{E}$ well remained. The wellhead was protected by a metal shed surrounded by a 6-ft high cyclone and barbed wire fence with a locked gate.

- The wellhead valves (Christmas tree), separator, and connecting piping at the R-EX well were left configured for future gas production. One drip pan was in place around the wellhead, and another was under the separator.

- A tool and instrument shed in the vicinity of the R-EX well was left.

- A large decontamination pan (old pipe rack pan) was left.

- Three 210-barrel water holding tanks and two 500-gallon hydrocarbon distillate tanks, all internally contaminated stayed. The water tanks contained a few inches of contaminated sludge solidified with bentonite. The hydrocarbon tanks were drained completely dry.

- Telephone facilities and electric power on boards and poles remained.

- The area was fenced with barbed wire and posted. 


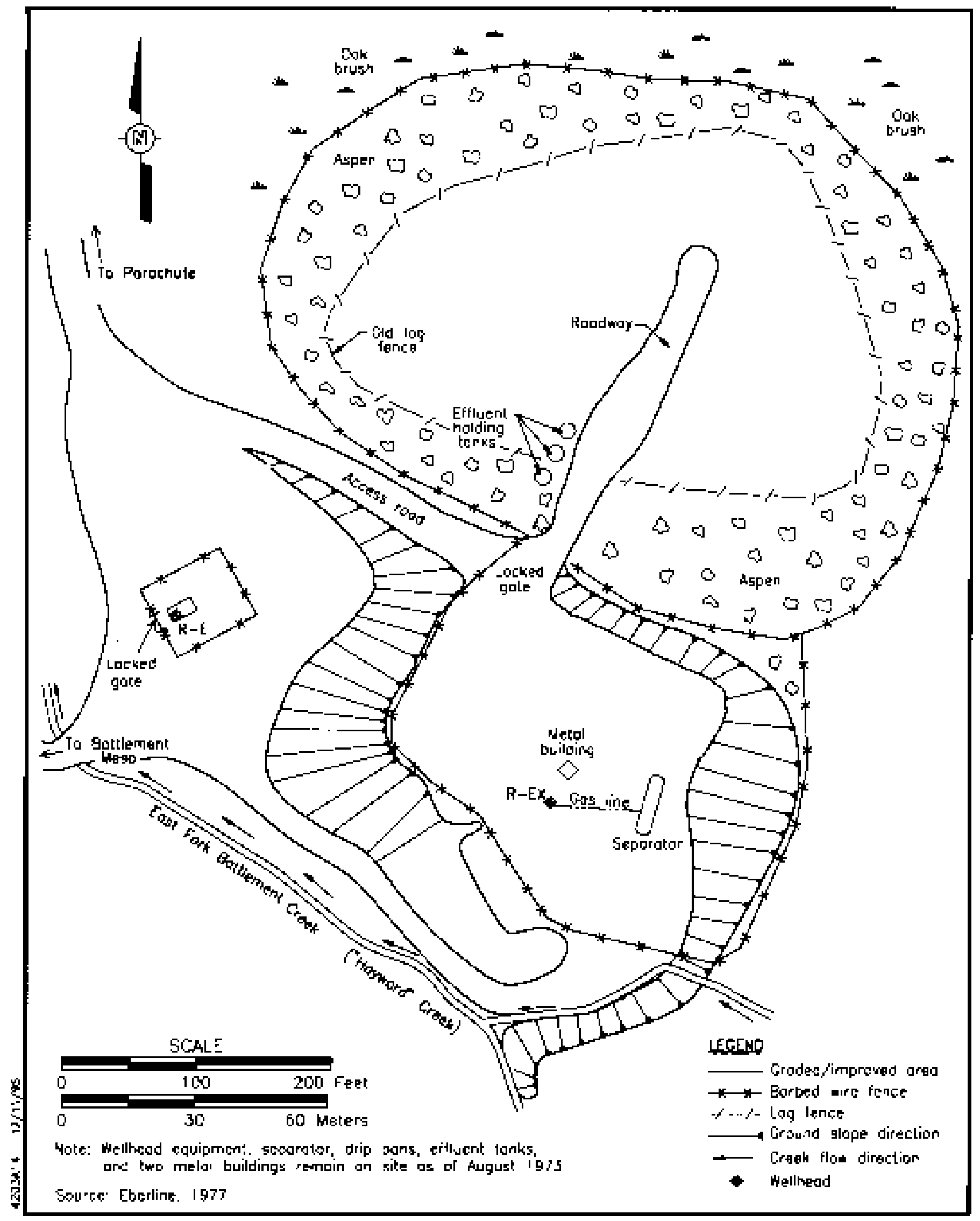

Figure 2-4

Pulison Site at Completion of the General Site Cleanup Effort, July 1972, Garfield County, Colorado 
Some of the above items were, or were presumed to be, contaminated internally. Items contaminated internally with tritium were appropriately labeled. None of these items was externally contaminated.

\subsubsection{Final Cleanup Effort (September 1 through October 12, 1976)}

The R-E and R-EX wells were plugged and abandoned during the final cleanup. Concurrently, the surface equipment (itemized in the general cleanup) was dismantled, decontaminated, documented in the release log, and removed from the site. The primary method of decontamination was by cleaning in a large, sheet-metal pan using saturated steam and Steamzall ${ }^{\circledR}$ or detergent. The only contaminant of concern was tritium. The guideline limit for release to unrestricted use was 5,000 disintegrations per minute $(\mathrm{dpm}) / 100 \mathrm{~cm}^{2}$ total activity and 1,000 $\mathrm{dpm} / 100 \mathrm{~cm}^{2}$ removable activity (ERDA, 1976). The release log listed 126 items for unrestricted use. No item was above the ambient area background when surveyed at approximately $1 \mathrm{~cm}$ with an HP-210 beta-gamma probe having less than a $7 \mathrm{mg} / \mathrm{cm}^{2}$ absorber. Removable contamination as determined by swipe sampling was in no case more than a small fraction of the guideline (ERDA, 1977, p. 3).

On October 4, 1976, $0.166 \mathrm{Ci}$ of tritium in waste water and drilling mud were pumped into the Mesaverde formation of the R-E well at a depth of approximately 1,615 to 1,768 $\mathrm{m}(5,300$ to $5,800 \mathrm{ft}$ ) for disposal. The potable aquifers above this depth were cemented off during well drilling and casing installation.

Items having inaccessible surfaces (i.e., pipes) were initially flushed with steam and cleaning solutions until flush liquids were below detection sensitivity for tritium. Following a drying period, an appropriate amount (not to exceed 1 liter) of distilled water was placed in contact with the portion of the surface to be tested. A one cubic centimeter $\left(\mathrm{cm}^{3}\right)$ aliquot of this water was collected and analyzed for tritium. If the concentration exceeded 5,000 dpm/milliliter ( $\mathrm{mL})$, the item was considered unfit for unconditional release. None of the decontaminated items exceeded this limit. The R-E wellhead equipment and metal shed were not contaminated and were released after the survey.

The R-EX wellhead, separator, and connecting pipeline were internally contaminated. The wellhead was disassembled so that the internal surfaces were accessible for steam cleaning. The pipeline was cut into manageable lengths which were cleaned internally with a steam lance. The separator was moved onto the decontamination pan where its pressure tanks were cut open with 
an acetylene torch so that internal surfaces were accessible for steam cleaning. The wellhead drip pan, the separator drip pan, and the tool shed were not contaminated.

The three water holding tanks were moved onto the decontamination pan. The heater of each was removed for decontamination and to obtain a large access port to the tank. Thirty 55-gallon steel drums of solidified sludge were mucked from the bottom of these tanks through the heater openings. The heaters and internal surfaces of the tanks were decontaminated with de-tar solvent, saturated steam, Steamzall ${ }^{\circledR}$, and detergent. The two hydrocarbon tanks had been transferred to Project Rio Blanco, and they were not included in the Rulison cleanup.

On October 8, 1976, as a result of the final cleanup, sixty-eight 55-gallon steel drums of contaminated soil and other solid waste containing a total of $0.018 \mathrm{Ci}$ of tritium were shipped to Beatty, Nevada, for burial at the Nuclear Engineering Company facility. This waste originated from mucking the tanks, soil removal of known spill areas, and from decontamination activities associated with drillback and flaring operations. The total amount of tritium shipped for burial from the Rulison Site as a result of both the general and final cleanup operations was estimated to be $0.781 \mathrm{Ci}$. No other radionuclide was involved in either cleanup.

\subsubsection{Plugging and Abandonment Operations}

The R-E and R-EX wells were plugged concurrently with the final cleanup work. The R-EX well was plugged first and the R-E well second. Both procedures required the use of a work-over drilling rig with routine support activities. Radiological monitoring support was provided to assure safety of personnel and containment of any radioactive material coming from downhole.

\subsection{R-EX Well}

This well was originally the pre-shot exploratory hole used to perform pre-shot gas-production tests, conducting geological and hydrological studies, and other studies for technical and safety confirmation. This well was also used for reentry and production testing (ERDA, 1976, p. 5). It was plugged pursuant to the plan (ERDA, 1976) (Figure 2-5). An unexpected return to the surface of 300 barrels of drilling mud and water contaminated with low levels of tritium was a potential source of contamination. However, this return was totally contained in tanks and was later disposed of, along with other liquids, as previously noted. 
Perforated 914.40 to 914.70 meter $(m)$

(3.000 Io 3,001 teel $[\mathrm{et}])$

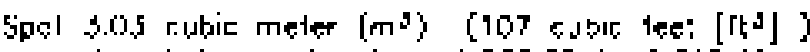
cernent - bolanse plug trom 9.905 .30 to $1.618 .49 \mathrm{~m}$ $(6,251105,3104)^{4}$

Pertareled 1,905.00 to 1.906.35 m $(5,250$ to 5,251 til)

5po1 $3.19 \pi^{3}$ (112.5 [t $\left.{ }^{3}\right)$ cemenl bolance glug frem 2,280.57 to $1,948.89$ r. $(7,515$ is 6.394 i: $)$

Perforated 3/2-in. tubing ot $2,275.33$ tc 2,275 64 m

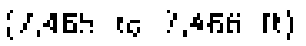

Gridge sug ngide 3 lh-in. 1ubing ot $2286.00 \mathrm{~m}$ (3.500 in

Pocker (permanenl') set al $2,290.57 \mathrm{~m}$ \{7,515 i:) S-' nipple ol $2.296 .9 / \mathrm{m}(1.5 .5541)$ $S-7$ ripple al $2.298 .4 \mathrm{~m}$ m 0.540

Note lype of rement used lor plugs ond squsere jobs was $50 \%$ reat $50 \%$ Pozmix. 27. प्रl, 47 HR-4. $7.891 .50 \mathrm{gram}(13+6)$ sock sol1

$18.28 \mathrm{~m}^{3}\left(645.5 \mathrm{ft}^{5}\right)$ cement spotyed ond citculaled hirauger perfarọlipns to till annular spoce ond $3 / 2-i n$. tubing to the surfoce - buianced plaç $9: 4.70 \mathrm{~m}\left(5.00^{\circ}\right.$ 1t) to surface

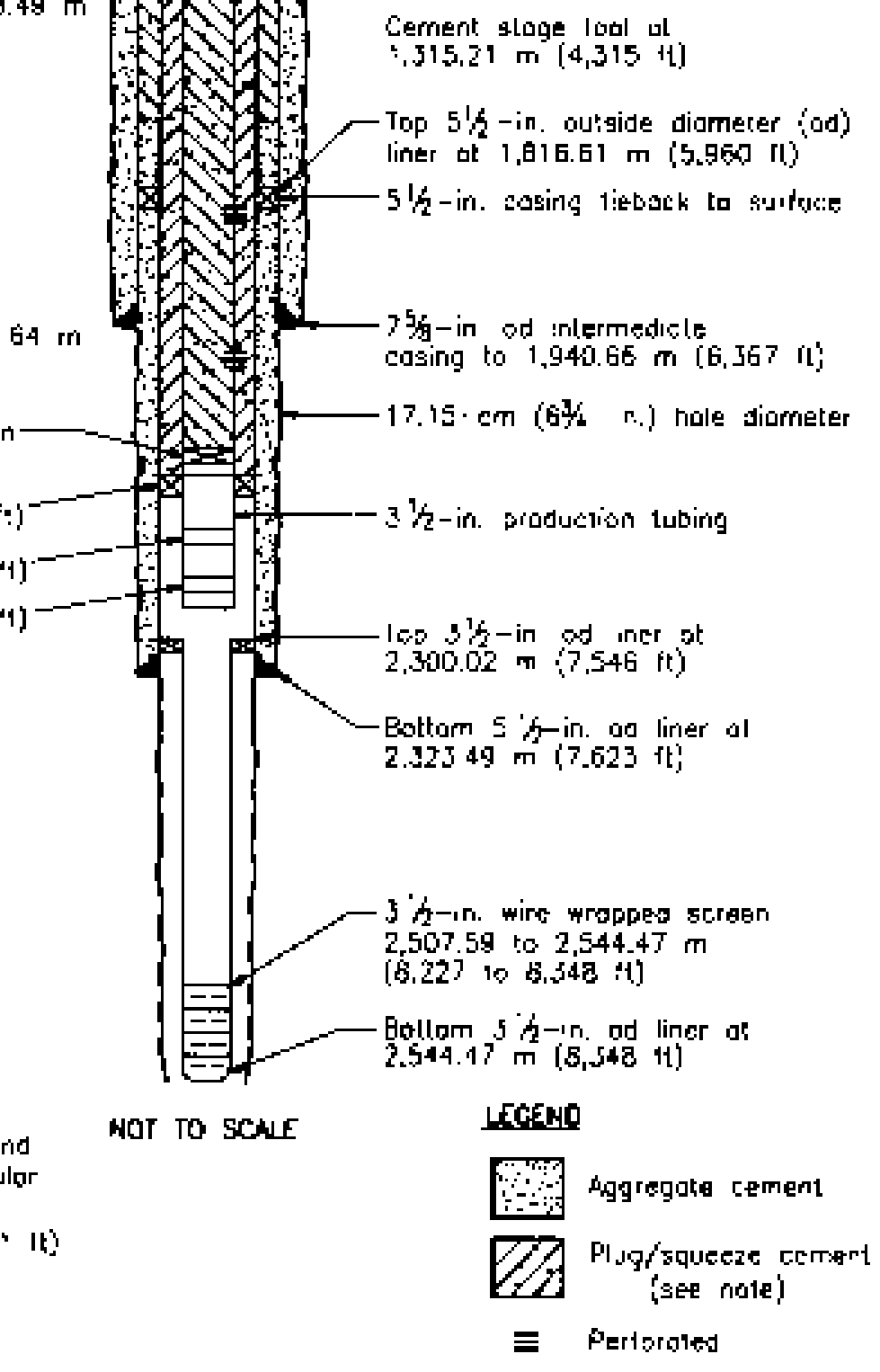

Sourct: LHDA, ISTI

Figure 2-5

$---$

Project Rullson Exploratory - Reantry Well (R-EX) "As Built" Plugging Conditlon, July 1972, Garfield County, Colorado 


\subsection{R-E Well}

The R-E Well contained stemming gravel and the nuclear device emplacement and detonation cable. There were several physical problems related to the washing out of stemming material and the removal of the cable (Figure 2-6). The original plan (ERDA, 1976) was modified by both regulatory decisions and practical demands. During the destemming operation, the return line of the wash-down fluid recirculating system was continuously monitored for gamma radiation with a 2-in. x 2-in. sodium iodide detector equipped with an alarm and recorder. A sample of the return fluid was collected at least every $36.58 \mathrm{~m}$ (120 ft) of depth and analyzed for tritium by liquid scintillation. Several samples of returned stemming material were analyzed for radioactive particulate contamination using pulse-height analysis. No radioactive contaminant above natural background was detected, and the well was satisfactorily plugged without a radiological incident.

\subsubsection{Environmental Sampling and Survey Programs}

Three environmental sampling programs were conducted. The first program was conducted after completion of production testing in 1971 and consisted of collecting soil samples from around the flare stack in a radial pattern. The second program was conducted in conjunction with the 1972 general cleanup. It included soil, vegetation, and water on and around the R-EX area, including more samples around the flare stack. The third program was part of the final cleanup in 1976 which was involved with well plugging and abandonment. It included extensive soil sampling in areas of known or potential contamination based on the results of prior sampling and operating experience. This program also included sampling the creek above and below the site as well as spring water at the site.

The three sampling programs adequately delineated the extent of soil and water contamination in the site area after completion of plugging procedures on the R-E and R-EX wells. The only radioactive nuclide in the environment of the site, other than those naturally occurring or resulting from worldwide fallout, was tritium. The final survey of tritium concentration did not exceed the guideline limit of $3 \times 10^{-2}$ microCuries per milliliter $(\mu \mathrm{Ci} / \mathrm{mL})\left(3 \times 10^{4}\right.$ picoCuries per milliliter [pCi/mL]) of soil moisture (ERDA, 1976).

After the final cleanup was completed, a survey of the site was made at 1-cm distance on a 15.24-m (50-ft grid) by 3.05-m ([10-ft] grid over areas of known spills) using an HP-210 beta-gamma probe having less than a $7 \mathrm{mg} / \mathrm{cm}^{2}$ absorber. No reading was obtained greater than the ambient background ( $0.02 \mathrm{mrad} /$ hour $)$ of the area. 


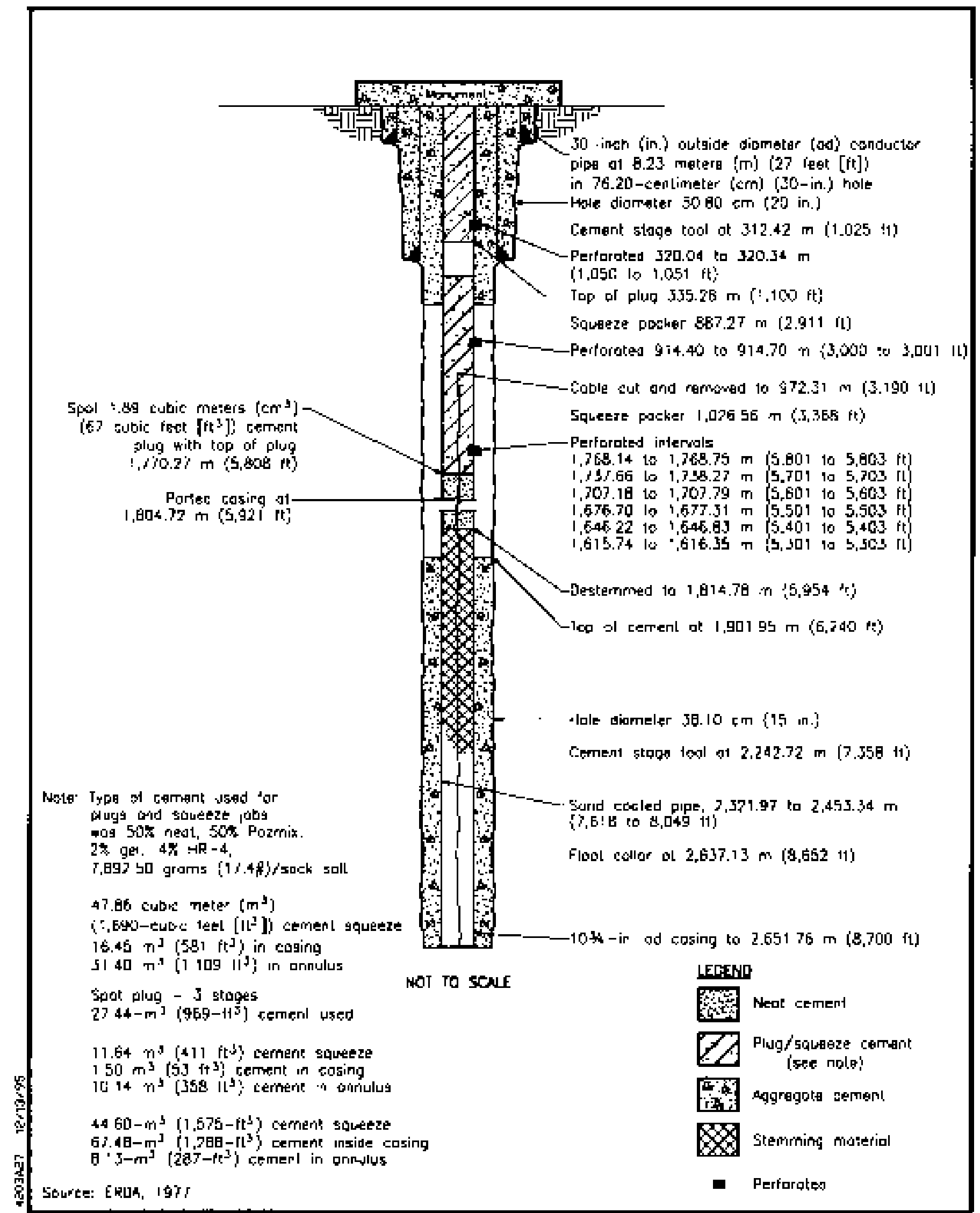

\section{Project Pulison, Emplacement Hole (R-E) "As Bultt" Plugging Conditlon, July 1972 , Garfield County, Colorado}




\subsection{First Sampling Program}

This first sampling program was conducted in April 1971 when the site was placed on standby after completion of production tests. A total of 133 soil samples was taken at 70 sampling points around the flare stack. All samples were well below the guidelines for tritium in soil moisture. Figures 2-7 and 2-8 show the locations of each sampling point by azimuth and distance from the flare stack and the tritium concentration in soil moisture per milliliter and per gram at the indicated sample depths. Table 2-1 provides the same information in tabular form.

\subsection{Second Sampling Program}

This program was part of the general cleanup conducted in July 1972. It included the sampling of soil, vegetation and water.

\section{Soil Sampling}

A square grid of soil sampling points was laid out on magnetic cardinal headings using the site entrance gate post as the zero and primary reference point. Ten- and twenty-foot squares were used, depending on the area use history and on the probability of soil contamination. Squares were sometimes distorted to sample points of special interest such as storage tanks, pipeline runs, the separator, and drip pan areas or to avoid obstructions such as cement pads. While the flare stack was located on the square grid system, the area around it was sampled on a radial grid referenced to the stack. This radial grid was used because contaminated fallout originated from the stack as a center and because a radial sampling grid was used previously during postflare operations, making a comparison more meaningful. A total of 192 sampling points was located (see Figures 2-9 and 2-10). Most of these points were sampled at 2.54-cm and 30.48-cm (1- and 12-in.) depths. Fourteen points were sampled at $2.54-\mathrm{cm}, 30.48-\mathrm{cm}, 60.96-\mathrm{cm}$, and $121.92-\mathrm{cm}$ (1-, 12-, 24-, and 48-in.) depths. Two points were sampled at multiple depths to $2.44 \mathrm{~m}$ and $3.35 \mathrm{~m}$ (96 and $132 \mathrm{in}$.), respectively, and a few were sampled at other selected depths. A total of 426 soil samples was collected for tritium analysis.

The depth increment for soil samples taken was $2.54 \mathrm{~cm}$ (1 in.) (i.e., the 2.54-cm sample was from the surface to $2.54 \mathrm{~cm}$, and the 30.48 -cm [12-in.] sample was from 27.94 to $30.48 \mathrm{~cm}$ [11 to 12 in.], etc.). Soil samples were collected in standard 454-gram (16-ounce) cottage cheese containers that held 61 to $68.6 \mathrm{~cm}^{3}$ (24 to 27 cubic in.) of sample. At undisturbed and uncompacted sampling locations, an earth auger was used to bore holes up to 1.22-m (4-ft) deep. For sampling at greater depths, and at disturbed and compacted locations, a powered backhoe was used to dig required holes. After these holes were cleaned out, samples were taken from 


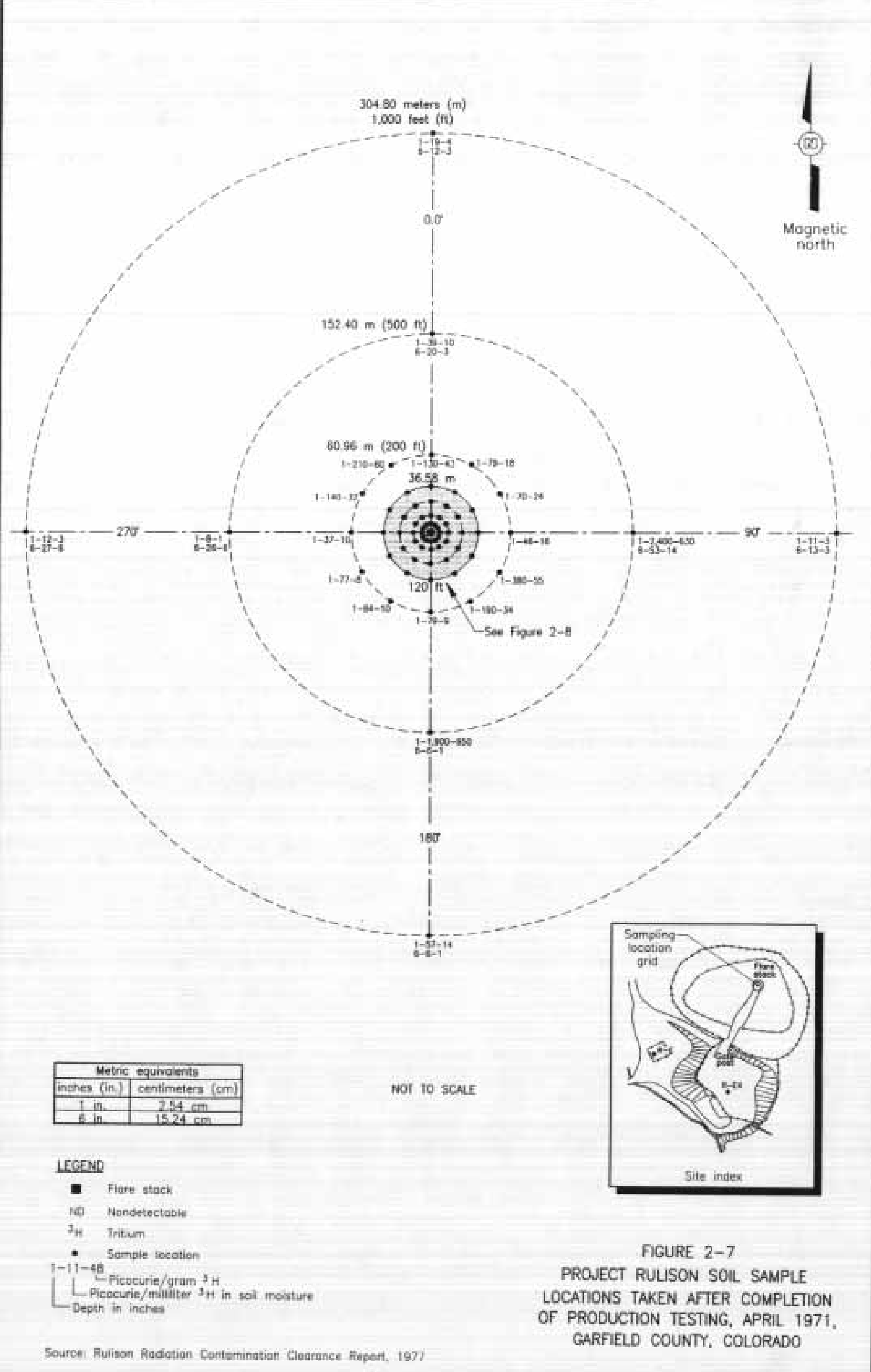




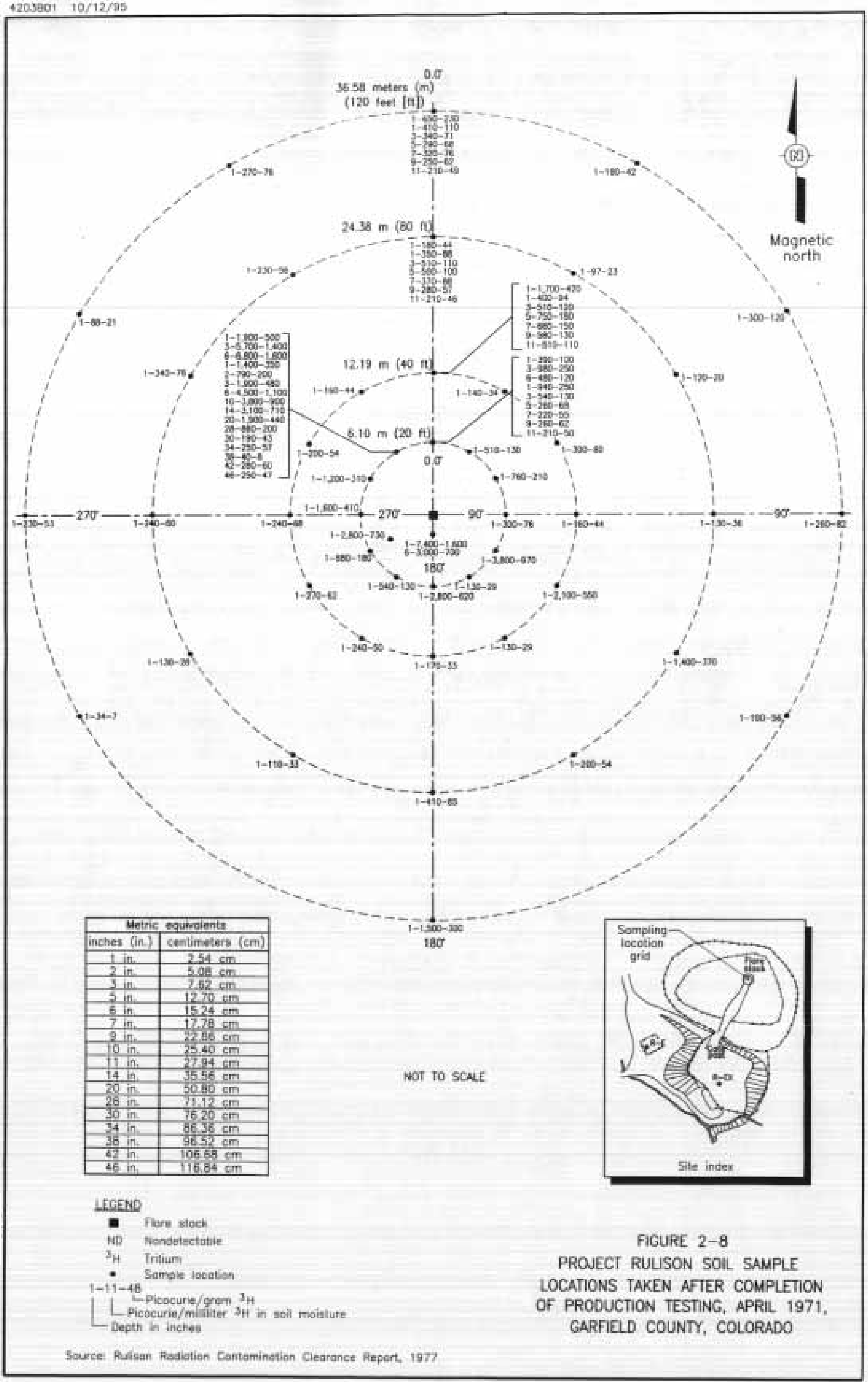




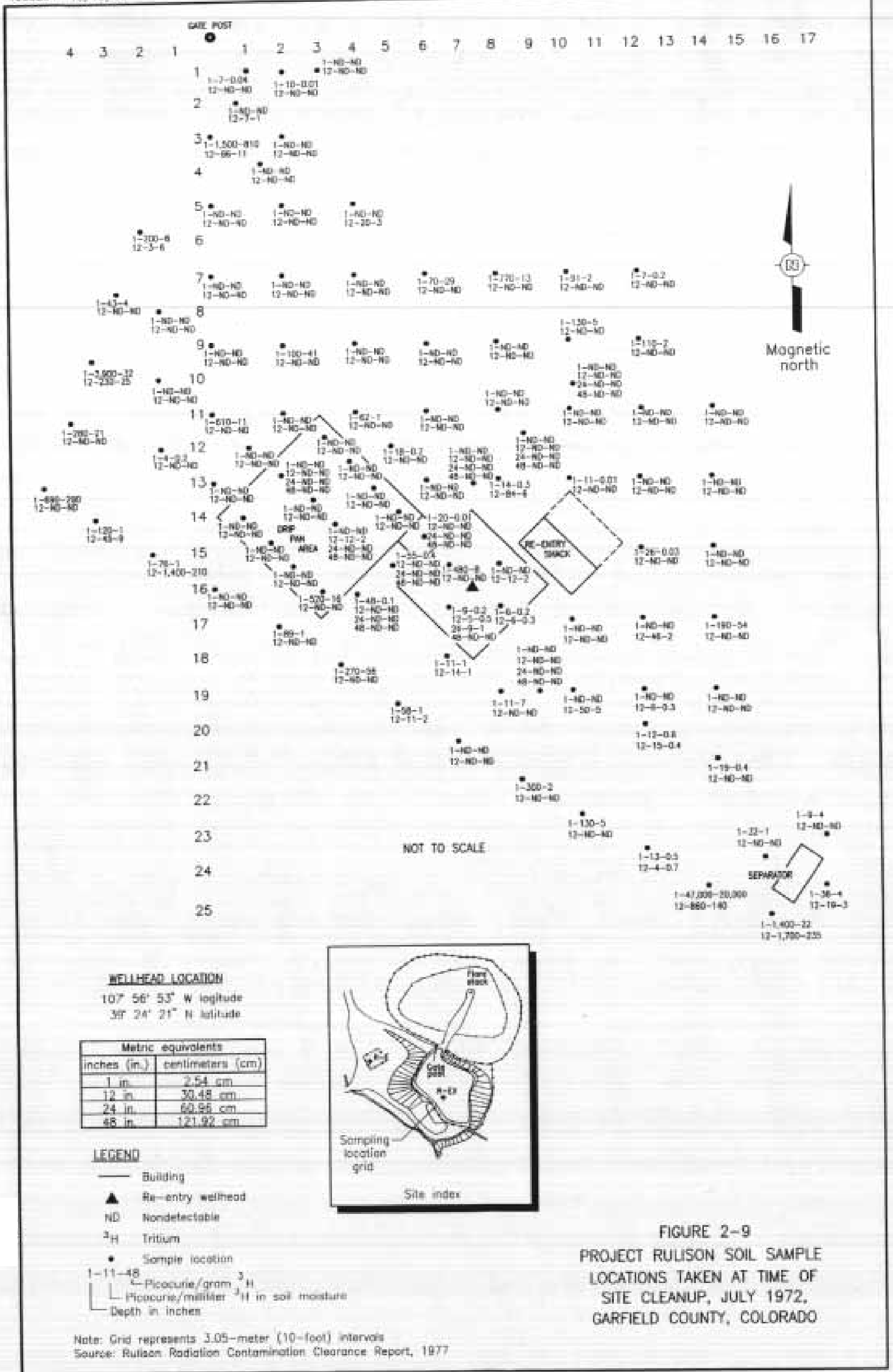


$=\frac{24-650-900}{60-7,000-4,400}$

$=\frac{1-11-2}{12-32-7}$

$=\frac{17+10-24}{17-25-22}$

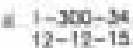

* $\mathfrak{l}_{i j-40-10}^{40-10}$

$1+24-8$

क t-150-5i

n $12-73-21$

$1,-10000-1,400$ $14-21000-100$

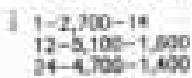

- $\begin{aligned} & 1-4,100-1.490 \\ & 12-1,300 \times 1900\end{aligned}$

$11-0.050-1900$

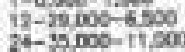

$34-4000-10,000$

0

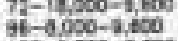

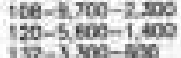

in $1-230-1000$

$24-3.00-1.00$

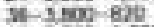

$-5 \times 0-10$

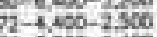

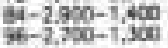

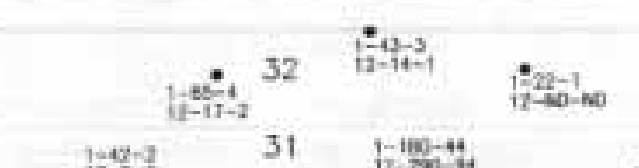

$$
\begin{aligned}
& 30
\end{aligned}
$$

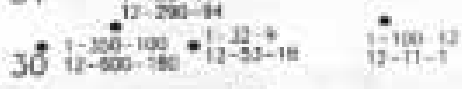

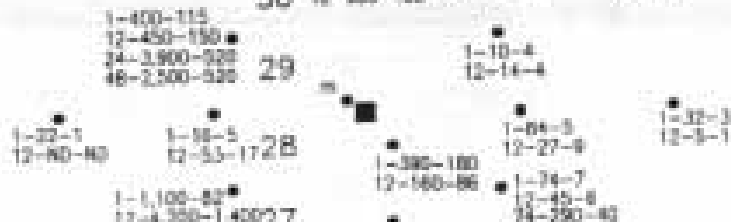

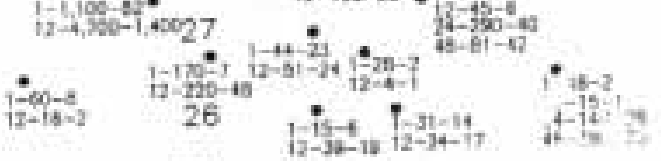

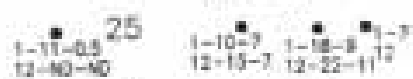

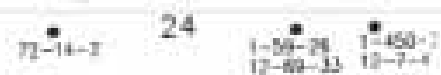$$
23+15-70.140 \text { 安 }
$$$$
22+\frac{1}{120}+1004
$$$$
21 \quad \operatorname{lin}_{12-72}-4 x
$$$$
20 \underset{12-400}{12-280}
$$$$
19 \quad \text { is } i=15-1
$$$$
18+\frac{18}{18-500-190}
$$

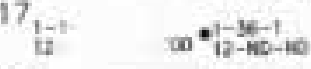$$
16 \quad: 025-11
$$

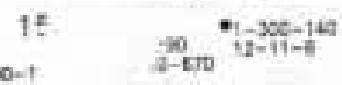

$1-x^{2}-2 \pi$
$12-13-4$

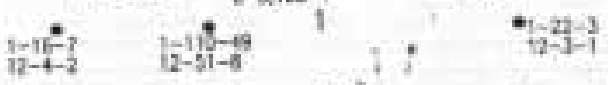

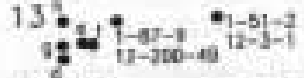

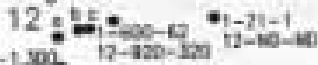

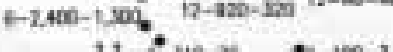

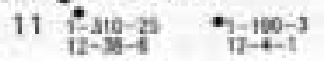

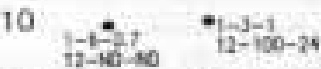

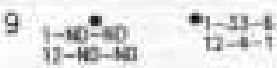

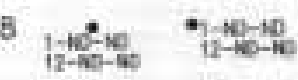

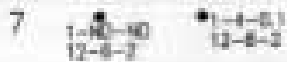

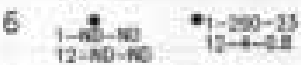

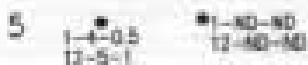

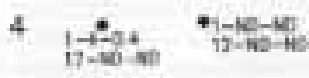

$$
\begin{aligned}
& 3 \lim _{2 \rightarrow-5}^{2}-10 \\
& 2 \quad \operatorname{lin}_{12-13-10} \\
& 0-\frac{1}{2}-0000
\end{aligned}
$$

\begin{tabular}{|c|c|}
\hline nches (in.) & centimeters $(\mathrm{cm})$ \\
\hline $1 \mathrm{in}$ & $254 \mathrm{~cm}$ \\
\hline 6 in & $15.24 \mathrm{~cm}$ \\
\hline 2 in. & $30.48 \mathrm{~cm}$ \\
\hline $24=$ & $60.5 \mathrm{~cm}$ \\
\hline $36 \mathrm{n}$ & $91.44 \mathrm{~cm}$ \\
\hline $4 B \pi$ & $17192 \mathrm{~cm}$ \\
\hline$\frac{60}{22}$ in & $\begin{array}{l}15240 \mathrm{~cm} \\
182.8 \mathrm{~cm}\end{array}$ \\
\hline & $21336 \mathrm{~cm}$ \\
\hline 95 in & $245.14 \mathrm{~cm}$ \\
\hline 100 in & $27432 \mathrm{~cm}$ \\
\hline $120 \mathrm{n}$ & $304.60 \mathrm{~cm}$ \\
\hline 132 in & $315.73 \mathrm{~cm}$ \\
\hline
\end{tabular}

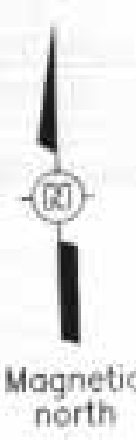

\section{LEGEND}

- Fiere Stack

ND Nondetectabie

Int Tritium
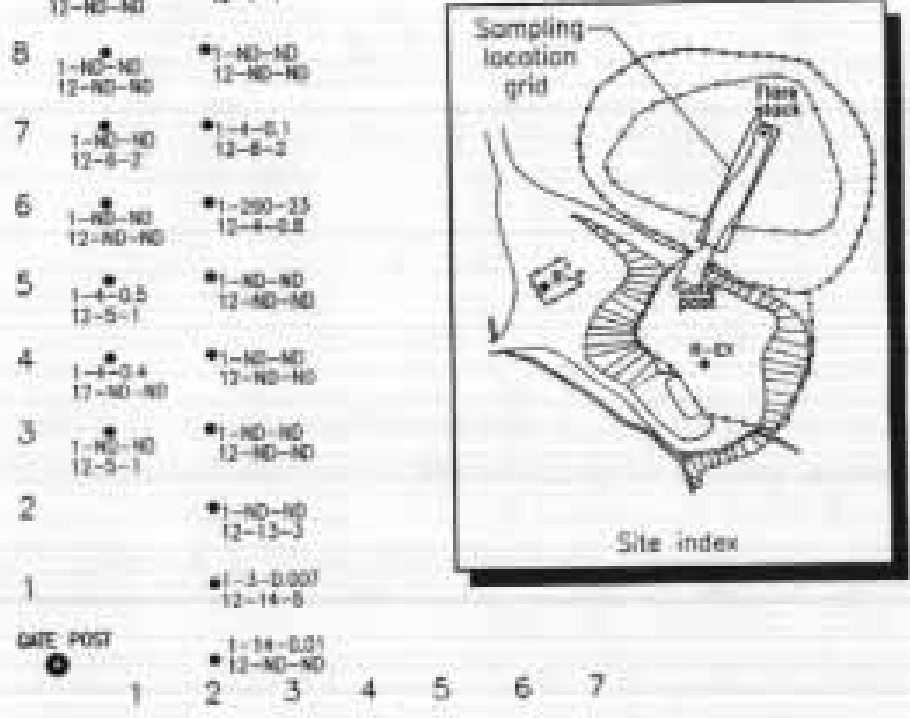

- Sompir iocation

$1=11-48$ picocurie/grom ${ }^{3} \mathrm{H}$

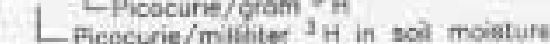

- Depth in nethes

Notk: Grid represents 105-meter (10-toot) miervai

NOT To SCALE

FIGURE $2-10$

PROJECT RUUSON SOIL SAMPLE LOCATIONS TAKEN AT TIME OF SITE CLEANUP, JULY 1972. GARFIELD COUNTY, COLORADO

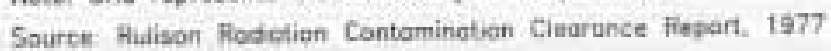


Table 2-1

Tritium in Rulison Soil Moisture Postproduction Test - April 23, 1971

(Page 1 of 4)

\begin{tabular}{|c|c|c|c|}
\hline $\begin{array}{c}\text { Grid Coordinates } \\
\text { degrees, meters (feet) }\end{array}$ & $\begin{array}{l}\text { Sampling Depth } \\
\text { centimeters (inches) }\end{array}$ & $\mathrm{pCi} / \mathrm{ml}^{\mathrm{c}}$ & pCi/g (soil) \\
\hline $000^{\circ}, 6.10(20)$ & $2.54(1)$ & 390 & 100 \\
\hline $000^{\circ}, 6.10(20)$ & $7.62(3)$ & 980 & 250 \\
\hline $000^{\circ}, 6.10(20)$ & $15.24(6)$ & 480 & 120 \\
\hline $000^{\circ}, 6.10(20)$ & 0 to 5.08 (0 to 2$)$ & 940 & 250 \\
\hline $000^{\circ}, 6.10(20)$ & 5.08 to 10.16 (2 to 4$)$ & 540 & 130 \\
\hline $000^{\circ}, 6.10(20)$ & 10.16 to 15.24 (4 to 6$)$ & 260 & 68 \\
\hline $000^{\circ}, 6.10(20)$ & 15.24 to 20.32 (6 to 8$)$ & 220 & 55 \\
\hline $000^{\circ}, 6.10(20)$ & 20.32 to 25.40 (8 to 10$)$ & 260 & 62 \\
\hline $000^{\circ}, 6.10(20)$ & 25.40 to 30.48 (10 to 12$)$ & 210 & 50 \\
\hline $000^{\circ}, 12.19(40)$ & $2.54(1)$ & 1,700 & 420 \\
\hline $000^{\circ}, 12.19(40)$ & 0 to 5.08 (0 to 2$)$ & 400 & 94 \\
\hline $000^{\circ}, 12.19(40)$ & 5.08 to $10.16(2$ to 4$)$ & 510 & 120 \\
\hline $000^{\circ}, 12.19(40)$ & 10.16 to 15.24 (4 to 6$)$ & 750 & 180 \\
\hline $000^{\circ}, 12.19(40)$ & 15.24 to $20.32(6$ to 8$)$ & 660 & 150 \\
\hline $000^{\circ}, 12.19(40)$ & 20.32 to 25.40 ( 8 to 10$)$ & 580 & 130 \\
\hline $000^{\circ}, 12.19(40)$ & 25.40 to 30.48 (10 to 12$)$ & 510 & 110 \\
\hline $000^{\circ}, 24.38(80)$ & $2.54(1)$ & 180 & 44 \\
\hline $000^{\circ}, 24.38(80)$ & 0 to 5.08 (0 to 2$)$ & 350 & 88 \\
\hline $000^{\circ}, 24.38(80)$ & 5.08 to $10.16(2$ to 4$)$ & 510 & 110 \\
\hline $000^{\circ}, 24.38(80)$ & 10.16 to $15.24(4$ to 6$)$ & 500 & 100 \\
\hline $000^{\circ}, 24.38(80)$ & 15.24 to 20.32 (6 to 8$)$ & 370 & 88 \\
\hline $000^{\circ}, 24.38(80)$ & 20.32 to 25.40 (8 to 10$)$ & 280 & 57 \\
\hline $000^{\circ}, 24.38(80)$ & 25.40 to 30.48 (10 to 12$)$ & 210 & 46 \\
\hline $000^{\circ}, 36.58(120)$ & $2.54(1)$ & 650 & 230 \\
\hline $000^{\circ}, 36.58(120)$ & 0 to 5.08 (0 to 2$)$ & 410 & 110 \\
\hline $000^{\circ}, 36.58(120)$ & 5.08 to $10.16(2$ to 4$)$ & 340 & 71 \\
\hline $000^{\circ}, 36.58(120)$ & 10.16 to 15.24 (4 to 6$)$ & 290 & 68 \\
\hline $000^{\circ}, 36.58(120)$ & 15.24 to 20.32 ( 6 to 8$)$ & 320 & 76 \\
\hline $000^{\circ}, 36.58(120)$ & 20.32 to 25.40 ( 8 to 10$)$ & 250 & 62 \\
\hline $000^{\circ}, 36.58(120)$ & 25.40 to 30.48 (10 to 12$)$ & 210 & 49 \\
\hline $000^{\circ}, 60.96(200)$ & $2.54(1)$ & 130 & 43 \\
\hline $000^{\circ}, 152: 40(500)$ & $2.54(1)$ & 39 & 9.8 \\
\hline $000^{\circ}, 152.40(500)$ & $15.24(6)$ & 20 & 3.4 \\
\hline $000^{\circ}, 304.80(1,000)$ & $2.54(1)$ & 19 & 4.1 \\
\hline $000^{\circ}, 304.80(1,000)$ & $15.24(6)$ & 12 & 2.7 \\
\hline $030^{\circ}, 6.10(20)$ & $2.54(1)$ & 510 & 130 \\
\hline $030^{\circ}, 12.19(40)$ & $2.54(1)$ & 140 & 34 \\
\hline $030^{\circ}, 60.96(200)$ & 2.54 (1) & 79 & 18 \\
\hline $030^{\circ}, 24.38(80)$ & $2.54(1)$ & 97 & 23 \\
\hline
\end{tabular}

Reier to footnotes at end of table. 
Table 2-1

Tritium in Rulison Soil Moisture Postproduction Test - April 23, 1971

(Page 2 of 4)

\begin{tabular}{|c|c|c|c|}
\hline $\begin{array}{c}\text { Grid Coordinates" } \\
\text { degrees, meters (feet) }\end{array}$ & $\begin{array}{c}\text { Sampling Depth } \\
\text { centimeters (inches) }\end{array}$ & $\mathrm{pCi} / \mathrm{ml}^{\mathrm{c}}$ & pCi/gd (soil) \\
\hline $030^{\circ}, 36.58(120)$ & $2.54(1)$ & 180 & 42 \\
\hline $060^{\circ}, 6.10(20)$ & $2.54(1)$ & 760 & 210 \\
\hline $060^{\circ}, 24.38(80)$ & $2.54(1)$ & 120 & 20 \\
\hline $060^{\circ}, 36.58(120)$ & $2.54(1)$ & 300 & 120 \\
\hline $060^{\circ}, 60.96(200)$ & $2.54(1)$ & 70 & 24 \\
\hline $090^{\circ}, 6.10(20)$ & $2.54(1)$ & 300 & 76 \\
\hline $090^{\circ}, 12.19(40)$ & $2.54(1)$ & 160 & 44 \\
\hline $090^{\circ}, 24.38(80)$ & $2.54(1)$ & 130 & 36 \\
\hline $090^{\circ}, 36.58(120)$ & $2.54(1)$ & 260 & 82 \\
\hline $090^{\circ}, 60.96(200)$ & $2.54(1)$ & 46 & 16 \\
\hline $090^{\circ}, 152.40(500)$ & $2.54(1)$ & 2,400 & 630 \\
\hline $090^{\circ}, 152.40(500)$ & $15.24(6)$ & 53 & 14 \\
\hline $090^{\circ}, 304.80(1,000)$ & $2.54(1)$ & 11 & 3.2 \\
\hline $090^{\circ}, 304.80(1,000)$ & $15.24(6)$ & 13 & 3.3 \\
\hline $120^{\circ}, 6.10(20)$ & $2.54(1)$ & 3,800 & 970 \\
\hline $120^{\circ}, 12.19(40)$ & $2.54(1)$ & 2,100 & 550 \\
\hline $120^{\circ}, 24.38(80)$ & $2.54(1)$ & 1,400 & 370 \\
\hline $120^{\circ}, 36.58(120)$ & $2.54(1)$ & 190 & 56 \\
\hline $120^{\circ}, 60.96(200)$ & $2.54(1)$ & 380 & 55 \\
\hline $150^{\circ}, 6.10(20)$ & $2.54(1)$ & 130 & 29 \\
\hline $150^{\circ}, 12.19(40)$ & $2.54(1)$ & 710 & 160 \\
\hline $150^{\circ}, 24.38(80)$ & $2.54(1)$ & 200 & 54 \\
\hline $150^{\circ}, 36.58(120)$ & 0 to $2.54(0$ to 1$)$ & 210 & 65 \\
\hline $150^{\circ}, 36.58(120)$ & 2.54 to 5.08 (1 to 2$)$ & 180 & 53 \\
\hline $150^{\circ}, 36.58(120)$ & 5.08 to $10.16(2$ to 4$)$ & 220 & 62 \\
\hline $150^{\circ}, 36.58(120)$ & 10.16 to 20.32 (4 to 8$)$ & 290 & 87 \\
\hline $150^{\circ}, 36.58(120)$ & 20.32 to 30.48 (8 to 12$)$ & 420 & 110 \\
\hline $150^{\circ}, 36.58(120)$ & 30.48 to 40.64 (12 to 16$)$ & 340 & 84 \\
\hline $150^{\circ}, 36.58(120)$ & 40.64 to 50.80 (16 to 20$)$ & 130 & 25 \\
\hline $150^{\circ}, 36.58(120)$ & 50.80 to 60.96 (20 to 24$)$ & 79 & 16 \\
\hline $150^{\circ}, 36.58(120)$ & 60.96 to 71.12 (24 to 28$)$ & 75 & 15 \\
\hline $150^{\circ}, 36.58(120)$ & 71.12 to 81.28 (28 to 32$)$ & 110 & 19 \\
\hline $150^{\circ}, 36.58(120)$ & 81.28 to 91.44 (32 to 36 ) & 110 & 22 \\
\hline $150^{\circ}, 36.58(120)$ & 91.44 to 101.60 (36 to 40$)$ & 87 & 19 \\
\hline $150^{\circ}, 36.58(120)$ & 101.60 to 111.76 (40 to 44$)$ & 62 & 14 \\
\hline $150^{\circ}, 36.58(120)$ & 111.76 to 121.92 (44 to 48 ) & 59 & 13 \\
\hline $150^{\circ}, 60.96(200)$ & $2.54(1)$ & 190 & 34 \\
\hline $180^{\circ}, 1.52(5)$ & $2.54(1)$ & 7,400 & 1600 \\
\hline $180^{\circ}, 1.52(5)$ & $15.24(6)$ & 3,000 & 700 \\
\hline
\end{tabular}

Refer to footnotes at end of table. 
Table 2-1

Tritium in Rulison Soil Moisture Postproduction Test - April 23, 1971 (Page 3 of 4)

\begin{tabular}{|c|c|c|c|}
\hline $\begin{array}{c}\text { Grid Coordinates } \\
\text { degrees, meters (feet) }\end{array}$ & $\begin{array}{c}\text { Sampling Depth } \\
\text { centimeters (inches) }\end{array}$ & $\mathrm{pCi} / \mathrm{ml}^{\mathrm{c}}$ & pCi/ga (soil) \\
\hline $180^{\circ}, 6.10(20)$ & $2.54(1)$ & 2,800 & 620 \\
\hline $180^{\circ}, 12.19(40)$ & $2.54(1)$ & 170 & 33 \\
\hline $180^{\circ}, 24.38(80)$ & $2.54(1)$ & 410 & 85 \\
\hline $180^{\circ}, 36.58(120)$ & $2.54(1)$ & 1,500 & 300 \\
\hline $180^{\circ}, 60.96(200)$ & $2.54(1)$ & 79 & 8.7 \\
\hline $180^{\circ}, 152.40(500)$ & $2.54(1)$ & 1,900 & 650 \\
\hline $180^{\circ}, 152.40(500)$ & $15.24(6)$ & 6 & 1.1 \\
\hline $180^{\circ}, 304.80(1,000)$ & $2.54(1)$ & 57 & 14 \\
\hline $180^{\circ}, 304.80(1,000)$ & $15.24(6)$ & 6 & 1.2 \\
\hline $240^{\circ}, 4.27(14)$ & $2.54(1)$ & 2,800 & 730 \\
\hline $240^{\circ}, 6.10(20)$ & $2.54(1)$ & 680 & 180 \\
\hline $240^{\circ}, 12.19(40)$ & $2.54(1)$ & 270 & 62 \\
\hline $240^{\circ}, 24.38(80)$ & $2.54(1)$ & 130 & 28 \\
\hline $240^{\circ}, 36.58(120)$ & $2.54(1)$ & 34 & 7 \\
\hline $240^{\circ}, 60.96(200)$ & $2.54(1)$ & 77 & 8.1 \\
\hline $270^{\circ}, 6.10(20)$ & $2.54(1)$ & 1,600 & 410 \\
\hline $270^{\circ}, 12.19(40)$ & $2.54(1)$ & 240 & 68 \\
\hline $270^{\circ}, 24.38(80)$ & $2.54(1)$ & 240 & 60 \\
\hline $270^{\circ}, 36.58(120)$ & $2.54(1)$ & 230 & 53 \\
\hline $270^{\circ}, 60.96(200)$ & $2.54(1)$ & 37 & 10 \\
\hline $270^{\circ}, 152.40(500)$ & $2.54(1)$ & 8 & 1.2 \\
\hline $270^{\circ}, 152.40(500)$ & $15.24(6)$ & 26 & 5.8 \\
\hline $270^{\circ}, 304.80(1,000)$ & $2.54(1)$ & 12 & 2.6 \\
\hline $270^{\circ}, 304.80(1,000)$ & $15.24(6)$ & 27 & 5.6 \\
\hline $300^{\circ}, 6.10(20)$ & $2.54(1)$ & 1,200 & 310 \\
\hline $300^{\circ}, 12.19(40)$ & $2.54(1)$ & 200 & 54 \\
\hline $300^{\circ}, 24.38(80)$ & $2.54(1)$ & 340 & 76 \\
\hline $300^{\circ}, 36.58(120)$ & $2.54(1)$ & 88 & 21 \\
\hline $300^{\circ}, 60.96(200)$ & $2.54(1)$ & 140 & 32 \\
\hline $330^{\circ}, 6.10(20)$ & $2.54(1)$ & 1,900 & 500 \\
\hline $330^{\circ}, 6.10(20)$ & $7.62(3)$ & 5,700 & 1400 \\
\hline $330^{\circ}, 6.10(20)$ & $15.24(6)$ & 6,800 & 1600 \\
\hline $330^{\circ}, 6.10(20)$ & $2.54(1)$ & 1,400 & 350 \\
\hline $330^{\circ}, 6.10(20)$ & $5.08(2)$ & 790 & 200 \\
\hline $330^{\circ}, 6.10(20)$ & 5.08 to 10.16 (2 to 4$)$ & 1,900 & 480 \\
\hline $330^{\circ}, 6.10(20)$ & 10.16 to 20.32 (4 to 8 ) & 4,500 & 1100 \\
\hline $330^{\circ}, 6.10(20)$ & 20.32 to 30.48 ( 8 to 12 ) & 3,800 & 900 \\
\hline $330^{\circ}, 6.10(20)$ & 30.48 to 40.64 (12 to 16$)$ & 3,100 & 710 \\
\hline $330^{\circ}, 6.10(20)$ & 40.64 to 60.96 ( 16 to 24$)$ & 1,900 & 440 \\
\hline
\end{tabular}

Refer to footnotes at end of table. 


\section{Table 2-1 \\ Tritium in Rulison Soil Moisture Postproduction Test - April 23, 1971 (Page 4 of 4)}

\begin{tabular}{|r|r|r|r|}
\hline $\begin{array}{c}\text { Grid Coordinates } \\
\text { degrees, meters (feet) }\end{array}$ & $\begin{array}{c}\text { Sampling Depth } \\
\text { centimeters (inches) }\end{array}$ & pCi/mi $^{\mathrm{c}}$ & pCi/g $^{d}$ (soil) \\
\hline \hline $330^{\circ}, 6.10(20)$ & 60.96 to $71.12(24$ to 28$)$ & 860 & 200 \\
\hline $330^{\circ}, 6.10(20)$ & 71.12 to $81.28(28$ to 32$)$ & 190 & 43 \\
\hline $330^{\circ}, 6.10(20)$ & 81.28 to $91.44(32$ to 36$)$ & 250 & 57 \\
\hline $330^{\circ}, 6.10(20)$ & 91.44 to $101.60(36$ to 40$)$ & 40 & 8.1 \\
\hline $330^{\circ}, 6.10(20)$ & 101.60 to $111.76(40$ to 44$)$ & 280 & 60 \\
\hline $330^{\circ}, 6.10(20)$ & 111.76 to $121.92(44$ to 48$)$ & 250 & 47 \\
\hline $330^{\circ}, 12.19(40)$ & $2.54(1)$ & 160 & 44 \\
\hline $330^{\circ}, 24.38(80)$ & $2.54(1)$ & 230 & 56 \\
\hline
\end{tabular}

Source: Eberline, 1977

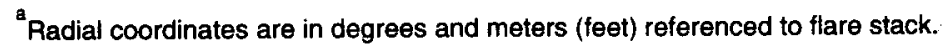

'Sampling depth increments, when not otherwise indicated, are $2.54 \mathrm{~cm}$ (i.e., $2.54 \mathrm{~cm}$ is from 0 to $2.54 \mathrm{~cm}$, $15.24 \mathrm{~cm}$ is from 12.70 to $15.24 \mathrm{~cm}$, etc.) ( $1^{*}$ [i.e., $1^{*}$ is from 0 to $1^{\prime \prime}, 6^{\prime \prime}$ is from $5^{\prime \prime}$ to $6^{\prime \prime}$, etc.]).

'Picocurie per milliliter

'Picocurie per gram 
their side walls at measured depths. Access to sampling points under waste water storage tanks was attained by drilling horizontally under each tank from a trench at its perimeter. Access to sampling points under drip pans was attained by cutting through the pan or by moving it to one side.

Each sample was weighed wet, as collected, and was then dried in an electric oven for 15 hours at 180 degrees centigrade. After drying, the sample was again weighed. Wet and dry weights were recorded for each sample, and the percentages of moisture were calculated. Where possible, a 5$\mathrm{mL}$ aliquot of soil moisture was distilled from each sample. The aliquots were analyzed by liquid scintillation for tritium concentration in $\mathrm{pCi} / \mathrm{mL}$. From this, the concentration in picoCuries per gram (pCi/g) was calculated. Results of these analyses are shown in Table 2-2.

Since no soil samples contained tritium above the concentration criterion of $3 \times 10^{4} \mathrm{pCi} / \mathrm{g}$, no soil was removed from the area.

Eight randomly located soil samples were collected for pulse height analysis by gamma spectrometry. No radioisotopes other than those naturally occurring were detected.

\section{Vegetation Sampling}

A vegetation sample was taken at each cardinal point on a 152-m, a 305-m (500-ft, and a 1,000-ft) arc around the flare stack. Additional vegetation samples were collected at site grid point N-14, W-2, and stack grid points $030^{\circ}, 5^{\prime}$ and $120^{\circ}, 40^{\prime}$. These samples were collected because of a leak from a water tank and a close proximity to the flare stack. This was the area of highest concentration as indicated by the post-flare sampling.

Vegetation samples were analyzed at Eberline Instrument Corporation's facilities in Albuquerque after the cleanup operation. Each sample was weighed wet and dry, and an aliquot of moisture was distilled from the sample. An aliquot of dry sample was oxidized and condensed to obtain the bound tritium. The results of these analyses are shown in Table 2-3.

\section{Water Sampling}

Prior to completion of the cleanup, water samples were taken from each of two local springs at the site. One was located just off the southeast corner of the R-EX well pad, the other was on the upper side of the road about $274 \mathrm{~m}$ (300 yards) downhill from the pad. Both samples were analyzed by liquid scintillation, and no tritium was detected. 
Table 2-2

Tritium in Rulison Soil Moisture - July 1972

(Page 1 of 9)

\begin{tabular}{|c|c|c|c|}
\hline Grid Coordinates $^{\mathrm{a}}$ & $\begin{array}{l}\text { Sampling Depth } \\
\text { centimeters (inches) }\end{array}$ & $\mathrm{pCi} / \mathrm{ml}^{\mathrm{c}}$ & $\mathrm{pCi} / g^{\alpha}$ (soil) \\
\hline $\mathrm{N}-0, \mathrm{E}-2$ & $2.54(1)$ & 14 & 0.01 \\
\hline $\mathrm{N}-0, \mathrm{E}-2$ & $30.48(12)$ & (5) ND & ND \\
\hline$N-1, E-2$ & $2.54(1)$ & 3.2 & 0.007 \\
\hline $\mathrm{N}-1, \mathrm{E}-2$ & $30.48(12)$ & 14 & 5.2 \\
\hline $\mathrm{N}-2, \mathrm{E}-2$ & $2.54(1)$ & $\mathrm{ND}$ & ND \\
\hline $\mathrm{N}-2, \mathrm{E}-2$ & $30.48(12)$ & 13 & 2.9 \\
\hline $\mathrm{N}-3, \mathrm{E}-.7$ & $2.54(1)$ & $\mathrm{ND}$ & $\overline{N D}$ \\
\hline $\mathrm{N}-3, \mathrm{E}-.7$ & $30.48(12)$ & 5.2 & 1.1 \\
\hline $\mathrm{N}-3, \mathrm{E}-2$ & $2.54(1)$ & ND & $\mathrm{ND}$ \\
\hline $\mathrm{N}-3, \mathrm{E}-2$ & $30.48(12)$ & $\mathrm{ND}$ & ND \\
\hline $\mathrm{N}-4, \mathrm{E}-.7$ & $2.54(1)$ & 3.8 & 0.4 \\
\hline $\mathrm{N}-4, \mathrm{E}-.27$ & $30.48(12)$ & $\mathrm{ND}$ & ND \\
\hline $\mathrm{N}-4, \mathrm{E}-2$ & $2.54(1)$ & ND & ND \\
\hline $\mathrm{N}-4, \mathrm{E}-2$ & $30.48(12)$ & ND & ND \\
\hline $\mathrm{N}-5, \mathrm{E}-.7$ & $2.54(1)$ & 4.3 & 0.5 \\
\hline $\mathrm{N}-5, \mathrm{E}-.7$ & $30.48(12)$ & 4.9 & 0.95 \\
\hline$N-5, E-2$ & $2.54(1)$ & ND & $\mathrm{ND}$ \\
\hline $\mathrm{N}-5, \mathrm{E}-2$ & $30.48(12)$ & ND & ND \\
\hline $\mathrm{N}-6, \mathrm{E}-.7$ & $2.54(1)$ & ND & $\mathrm{ND}$ \\
\hline $\mathrm{N}-6, \mathrm{E}-2$ & $2.54(1)$ & 290 & 23 \\
\hline $\mathrm{N}-6, \mathrm{E}-2$ & $30.48(12)$ & 4 & 0.8 \\
\hline $\mathrm{N}-7, \mathrm{E}-\mathrm{.7}$ & $2.54(1)$ & ND & ND \\
\hline $\mathrm{N}-7, \mathrm{E}-.7$ & $30.48(12)$ & 5.9 & $\overline{2}$ \\
\hline$N-7, E-2$ & $2.54(1)$ & 3.9 & 0.1 \\
\hline $\mathrm{N}-7, \mathrm{E}-2$ & $30.48(12)$ & 8.3 & 1.8 \\
\hline $\mathrm{N}-8, \mathrm{E}-.7$ & $2.54(1)$ & $\mathrm{ND}$ & ND \\
\hline $\mathrm{N}-8, \mathrm{E}-.7$ & $30.48(12)$ & ND & $\overline{N D}$ \\
\hline $\mathrm{N}-8, \mathrm{E}-2$ & $2.54(1)$ & $\mathrm{ND}$ & ND \\
\hline $\mathrm{N}-8, \mathrm{E}-2$ & $30.48(12)$ & $\mathrm{ND}$ & $\mathrm{ND}$ \\
\hline $\mathrm{N}-9, \mathrm{E}-.7$ & $2.54(1)$ & $\mathrm{ND}$ & $\overline{N D}$ \\
\hline $\mathrm{N}-9, \mathrm{E}-.7$ & $30.48(12)$ & ND & ND \\
\hline N-9, E-2 & $2.54(1)$ & 33 & 5.8 \\
\hline $\mathrm{N}-9, \mathrm{E}-2$ & $30.48(12)$ & 4.2 & 0.9 \\
\hline $\mathrm{N}-10, \mathrm{E}-\mathrm{.7}$ & $2.54(1)$ & 6.1 & 0.68 \\
\hline $\mathrm{N}-10, \mathrm{E}-.7$ & $30.48(12)$ & $\mathrm{ND}$ & ND \\
\hline $\mathrm{N}-10, \mathrm{E}-2$ & $2.54(1)$ & 2.8 & 0.08 \\
\hline $\mathrm{N}-10, \mathrm{E}-2$ & $30.48(12)$ & 100 & 24 \\
\hline $\mathrm{N}-11, \mathrm{E}-2$ & $2.54(1)$ & 190 & 2.8 \\
\hline $\mathrm{N}-11, \mathrm{E}-2$ & $30.48(12)$ & 4.1 & 0.9 \\
\hline $\mathrm{N}-11.2, \mathrm{E}-\mathrm{2}$ & $2.54(1)$ & 310 & 25 \\
\hline $\mathrm{N}-11.2, \mathrm{E}-.2$ & $30.48(12)$ & 38 & 6 \\
\hline $\mathrm{N}-11.4, \mathrm{E}-0$ & $15.24(6)$ & 2,400 & 1,300 \\
\hline $\mathrm{N}-11.8, \mathrm{E}-0$ & $60.96(24)$ & 850 & 510 \\
\hline $\mathrm{N}-11.8, \mathrm{E}-0$ & $152.40(60)$ & 7,800 & 4,400 \\
\hline $\mathrm{N}-11.9, \mathrm{E}-2.8$ & $2.54(1)$ & 11 & 2.3 \\
\hline $\mathrm{N}-11.9, \mathrm{E}-2.8$ & $30.48(12)$ & 33 & 6.9 \\
\hline $\mathrm{N}-11.9, \mathrm{E}-3.3$ & $2.54(1)$ & 110 & 24 \\
\hline $\mathrm{N}-11.9, \mathrm{E}-3.3$ & $30.48(12)$ & 93 & 22 \\
\hline $\mathrm{N}-12, \mathrm{E}-\mathrm{.}$ & $2.54(1)$ & 600 & 62 \\
\hline $\mathrm{N}-12, \mathrm{E}-\mathrm{.7}$ & $30.48(12)$ & 920 & 320 \\
\hline $\mathrm{N}-12, \mathrm{E}-2$ & $2.54(1)$ & 21 & 1 \\
\hline
\end{tabular}


Table 2-2

Tritium in Rulison Soil Moisture - July 1972

(Page 2 of 9)

\begin{tabular}{|c|c|c|c|}
\hline Grid Coordinates" & $\begin{array}{c}\text { Sampling Depth } \\
\text { centimeters (inches) }\end{array}$ & $\mathrm{pCi} / \mathrm{ml}^{\mathrm{c}}$ & pCi/g (soil) \\
\hline $\mathrm{N}-12, \mathrm{E}-2$ & $30.48(12)$ & $\overline{\mathrm{ND}}$ & $\overline{\mathrm{ND}}$ \\
\hline $\mathrm{N}-12.5, \mathrm{E}-0$ & $2.54(1)$ & 300 & 34 \\
\hline $\mathrm{N}-12.5, \mathrm{E}-0$ & $30.48(12)$ & 120 & 15 \\
\hline $\mathrm{N}-12.7, \mathrm{E}-0$ & $15.24(6)$ & 150 & 81 \\
\hline $\mathrm{N}-12.7, \mathrm{E}-2.8$ & $2.54(1)$ & 44 & 10 \\
\hline $\mathrm{N}-12.7, \mathrm{E}-2.8$ & $30.48(12)$ & ND & ND \\
\hline $\mathrm{N}-12.7, \mathrm{E}-3.3$ & $2.54(1)$ & 21 & 8.3 \\
\hline $\mathrm{N}-12.7, \mathrm{E}-3.3$ & $30.48(12)$ & 11 & 4.7 \\
\hline $\mathrm{N}-13, \mathrm{E}-\mathrm{0}$ & $30.48(12)$ & 73 & 41 \\
\hline $\mathrm{N}-13, \mathrm{E}-\mathrm{0}$ & $152.40(60)$ & 51 & 24 \\
\hline $\mathrm{N}-13, \mathrm{E}-\mathrm{.7}$ & $2.54(1)$ & 87 & 9.3 \\
\hline $\mathrm{N}-13, \mathrm{E}-.7$ & $30.48(12)$ & 200 & 49 \\
\hline $\mathrm{N}-13, \mathrm{E}-2$ & $2.54(1)$ & 51 & 2.4 \\
\hline $\mathrm{N}-13, \mathrm{E}-2$ & $30.48 \cdot(12)$ & 2.9 & 0.6 \\
\hline$N-13, W-3$ & $2.54(1)$ & 57 & 27 \\
\hline$N-13, W-3$ & $30.48(12)$ & 13 & 6.2 \\
\hline $\mathrm{N}-13.7, \mathrm{E}-.1$ & $2.54(1)$ & 10,000 & 1,400 \\
\hline $\mathrm{N}-13.7, \mathrm{E}-.1$ & $30.48(12)$ & 20,000 & 5,600 \\
\hline $\mathrm{N}-13.7, \mathrm{E}-.1$ & $60.96(24)$ & 21,000 & 5,800 \\
\hline $\mathrm{N}-13.7, \mathrm{E}-.6$ & $2.54(1)$ & 2,700 & 150 \\
\hline $\mathrm{N}-13.7, \mathrm{E}-.6$ & $30.48(12)$ & 5,100 & 1,600 \\
\hline $\mathrm{N}-13.7, \mathrm{E}-\mathrm{6}$ & $60.96(24)$ & 4,700 & 1,400 \\
\hline$N-14, E-0$ & $2.54(1)$ & 4,700 & 1,400 \\
\hline $\mathrm{N}-14, \mathrm{E}-0$ & $30.48(12)$ & 3,300 & 1,800 \\
\hline $\mathrm{N}-14, \mathrm{E}-2$ & $2.54(1)$ & 22 & 0.3 \\
\hline $\mathrm{N}-14, \mathrm{E}-2$ & $30.48(12)$ & 3.3 & 0.7 \\
\hline $\mathrm{N}-14.2, \mathrm{E}-\mathrm{.}$ & $2.54(1)$ & 8,600 & 1,500 \\
\hline $\mathrm{N}-14.2, \mathrm{E}-.7$ & $30.48(12)$ & 29,000 & 6,500 \\
\hline $\mathrm{N}-14.2, \mathrm{E}-.7$ & $60.96(24)$ & 35,000 & 11,000 \\
\hline $\mathrm{N}-14.2, \mathrm{E}-.7$ & $91.44(36)$ & 34,000 & 19,000 \\
\hline $\mathrm{N}-14.2, \mathrm{E}-.7$ & $121.92(48)$ & 27,000 & 7,300 \\
\hline $\mathrm{N}-14.2, \mathrm{E}-.7$ & $152.40(60)$ & 26,000 & 14,000 \\
\hline $\mathrm{N}-14.2, \mathrm{E}-.7$ & $182.88(72)$ & 18,000 & 9,600 \\
\hline $\mathrm{N}-14.2, \mathrm{E}-.7$ & $243.84(96)$ & 8,000 & 4,500 \\
\hline $\mathrm{N}-14.2, \mathrm{E}-.7$ & $274.32(108)$ & 9,700 & 2,300 \\
\hline $\mathrm{N}-14.2, \mathrm{E}-.7$ & $304.80(120)$ & 5,600 & 1,400 \\
\hline $\mathrm{N}-14.2, \mathrm{E}-.7$ & $335.28(132)$ & 3,300 & 600 \\
\hline $\mathrm{N}-14, \mathrm{~W}-2$ & $2.54(1)$ & 110 & 49 \\
\hline $\mathrm{N}-14, \mathrm{~W}-2$ & $30.48(12)$ & 51 & 7.7 \\
\hline$N-14, W-4$ & $2.54(1)$ & 16 & 7.3 \\
\hline$N-14, W-4$ & $30.48(12)$ & 4.4 & 2.1 \\
\hline $\mathrm{N}-14.2, \mathrm{E}-\mathrm{0}$ & $15.24(6)$ & 3,100 & 1,700 \\
\hline $\mathrm{N}-15, \mathrm{E}-1$ & $2.54(1)$ & 650 & 290 \\
\hline $\mathrm{N}-15, \mathrm{E}-1$ & $30.48(12)$ & 1,400 & 670 \\
\hline $\mathrm{N}-15, \mathrm{E}-2$ & $2.54(1)$ & 300 & 140 \\
\hline $\mathrm{N}-15, \mathrm{E}-2$ & $30.48(12)$ & 11 & 6 \\
\hline $\mathrm{N}-15, \mathrm{~W}-3$ & $2.54(1)$ & 420 & 22 \\
\hline $\mathrm{N}-15, \mathrm{~W}-3$ & $30.48(12)$ & 130 & 16 \\
\hline $\mathrm{N}-16, \mathrm{E}-1$ & $2.54(1)$ & 270 & 120 \\
\hline $\mathrm{N}-16, \mathrm{E}-1$ & $30.48(12)$ & 260 & 140 \\
\hline $\mathrm{N}-16, \mathrm{E}-2$ & $2.54(1)$ & 26 & 11 \\
\hline
\end{tabular}

Refer to footnotes at end of table. 


\section{Table 2-2}

Tritium in Rulison Soil Moisture - July 1972 (Page 3 of 9)

\begin{tabular}{|c|c|c|c|}
\hline Grid Coordinates $^{\mathrm{a}}$ & $\begin{array}{l}\text { Sampling Depth } \\
\text { centimeters (inches) }\end{array}$ & $\mathrm{pCi} / \mathrm{ml}^{\mathrm{c}}$ & pCi/g (soil) \\
\hline $\mathrm{N}-16, \mathrm{E}-2$ & $30.48(12)$ & 5.3 & 2.6 \\
\hline $\mathrm{N}-17, \mathrm{E}-1$ & $2.54(1)$ & 160 & 75 \\
\hline $\mathrm{N}-17, \mathrm{E}-1$ & $30.48(12)$ & 17,000 & 6,000 \\
\hline $\mathrm{N}-16.7, \mathrm{E}-2$ & $2.54(1)$ & 36 & 0.9 \\
\hline $\mathrm{N}-16.7, \mathrm{E}-2$ & $30.48(12)$ & ND & ND \\
\hline $\mathrm{N}-17.8, \mathrm{E}-2$ & $2.54(1)$ & 710 & 370 \\
\hline $\mathrm{N}-17.8, \mathrm{E}-2$ & $30.48(12)$ & 330 & 170 \\
\hline$N-18, E-1$ & $2.54(1)$ & 11 & 5.3 \\
\hline$N-18, E-1$ & $30.48(12)$ & 80 & 41 \\
\hline $\mathrm{N}-19, \mathrm{E}-1$ & $2.54(1)$ & 25 & 12 \\
\hline $\mathrm{N}-19, \mathrm{E}-1$ & $30.48(12)$ & 22 & 11 \\
\hline $\mathrm{N}-19, \mathrm{E}-2$ & $2.54(1)$ & 10 & 4.5 \\
\hline $\mathrm{N}-19, \mathrm{E}-2$ & $30.48(12)$ & 15 & 7.1 \\
\hline $\mathrm{N}-20, \mathrm{E}-1$ & $2.54(1)$ & 8.4 & 3.9 \\
\hline $\mathrm{N}-20, \mathrm{E}-1$ & $30.48(12)$ & 280 & 130 \\
\hline $\mathrm{N}-20, \mathrm{E}-2$ & $2.54(1)$ & 71 & 34 \\
\hline $\mathrm{N}-20, \mathrm{E}-2$ & $30.48(12)$ & 10 & 4.6 \\
\hline $\mathrm{N}-21, \mathrm{E}-1$ & $2.54(1)$ & 44 & 20 \\
\hline $\mathrm{N}-21, \mathrm{E}-1$ & $30.48(12)$ & 73 & 30 \\
\hline $\mathrm{N}-21, \mathrm{E}-2$ & $2.54(1)$ & 56 & 25 \\
\hline $\mathrm{N}-21, \mathrm{E}-2$ & $30.48(12)$ & $\mathrm{ND}$ & ND \\
\hline $\mathrm{N}-22, \mathrm{E}-1$ & $2.54(1)$ & 25 & 12 \\
\hline $\mathrm{N}-22, \mathrm{E}-1$ & $30.48(12)$ & 100 & 43 \\
\hline $\mathrm{N}-22, \mathrm{E}-2$ & $2.54(1)$ & 8.4 & 3.9 \\
\hline $\mathrm{N}-22, \mathrm{E}-2$ & $30.48(12)$ & 23 & 12 \\
\hline $\mathrm{N}-23, \mathrm{E}-1$ & $2.54(1)$ & 15 & 6.8 \\
\hline $\mathrm{N}-23, \mathrm{E}-1$ & $30.48(12)$ & 290 & 140 \\
\hline $\mathrm{N}-23, \mathrm{E}-2$ & $2.54(1)$ & 6.6 & 3.2 \\
\hline $\mathrm{N}-23, \mathrm{E}-2$ & $30.48(12)$ & 3.4 & 1.7 \\
\hline $\mathrm{N}-24, \mathrm{E}-1$ & $2.54(1)$ & 59 & 26 \\
\hline $\mathrm{N}-24, \mathrm{E}-1$ & $30.48(12)$ & 69 & 33 \\
\hline $\mathrm{N}-24, \mathrm{E}-2$ & $2.54(1)$ & 450 & 220 \\
\hline $\mathrm{N}-24, \mathrm{E}-2$ & $30.48(12)$ & 6.9 & 3.6 \\
\hline $\mathrm{N}-24, \mathrm{~W}-2$ & $182.88(72)$ & 14 & 2.1 \\
\hline $\mathrm{N}-25, \mathrm{E}-1$ & 2.54 (1) & 16 & 7.1 \\
\hline $\mathrm{N}-25, \mathrm{E}-1$ & $30.48(12)$ & 15 & 7.3 \\
\hline $\mathrm{N}-25, \mathrm{E}-2$ & $2.54(1)$ & 18 & 8.7 \\
\hline $\mathrm{N}-25, \mathrm{E}-2$ & $30.48(12)$ & 22 & 11 \\
\hline $\mathrm{N}-26, \mathrm{E}-1$ & $2.54(1)$ & 15 & 6.4 \\
\hline $\mathrm{N}-26, \mathrm{E}-1$ & $30.48(12)$ & 39 & 19 \\
\hline $\mathrm{N}-26, \mathrm{E}-2$ & $2.54(1)$ & 31 & 14 \\
\hline $\mathrm{N}-26, \mathrm{E}-2$ & $30.48(12)$ & 34 & 17 \\
\hline $\mathrm{N}-27, \mathrm{E}-1$ & $2.54(1)$ & 44 & 23 \\
\hline $\mathrm{N}-27, \mathrm{E}-1$ & $30.48(12)$ & 51 & 24 \\
\hline $\mathrm{N}-28, \mathrm{E}-1$ & $2.54(1)$ & 390 & 180 \\
\hline $\mathrm{N}-28, \mathrm{E}-1$ & $30.48(12)$ & 160 & 86 \\
\hline & & & \\
\hline $000^{\circ}, 6.10(20)$ & $2.54(1)$ & 180 & 44 \\
\hline $000^{\circ}, 6.10(20)$ & $30.48(12)$ & 290 & 94 \\
\hline $000^{\circ}, 12.19(40)$ & $2.54(1)$ & 43 & 3.2 \\
\hline
\end{tabular}


Table 2-2

Tritium in Rulison Soil Moisture - July 1972

(Page 4 of 9 )

\begin{tabular}{|c|c|c|c|}
\hline Grid Coordinates" & $\begin{array}{l}\text { Sampling Depth } \\
\text { centimeters (inches) }\end{array}$ & $\mathrm{pCi} / \mathrm{ml}^{\mathrm{c}}$ & $\mathrm{pCi} / \mathrm{g}^{\mathrm{d}}$ (soil) \\
\hline $000^{\circ}, 12.19(40)$ & $30.48(12)$ & 14 & 1.1 \\
\hline $030^{\circ}, 6.10(20)$ & $2.54(1)$ & 32 & 8.5 \\
\hline $030^{\circ}, 6.10(20)$ & $30.48(12)$ & 53 & 18 \\
\hline $030^{\circ}, 12.19(40)$ & $2.54(1)$ & 22 & $\overline{1.1}$ \\
\hline $030^{\circ}, 12.19(40)$ & $30.48(12)$ & ND & ND \\
\hline $060^{\circ}, 6.10(20)$ & $2.54(1)$ & 10 & 3.5 \\
\hline $060^{\circ}, 6.10(20)$ & $30.48(12)$ & 14 & 4.3 \\
\hline $060^{\circ}, 12.19(40)$ & $2.54(1)$ & 100 & 12 \\
\hline $060^{\circ}, 12.19(40)$ & $30.48(12)$ & 11 & 0.75 \\
\hline $090^{\circ}, 6.10(20)$ & $2.54(1)$ & 84 & 4.9 \\
\hline $090^{\circ}, 6.10(20)$ & $30.48(12)$ & 27 & 8.6 \\
\hline $090^{\circ}, 12.19(40)$ & $2.54(1)$ & 32 & 3 \\
\hline $090^{\circ}, 12.19(40)$ & $30.48(12)$ & 4.8 & 0.55 \\
\hline $120^{\circ}, 6.10(20)$ & $2.54(1)$ & 74 & 7.3 \\
\hline $120^{\circ}, 6.10(20)$ & $30.48(12)$ & 45 & 6 \\
\hline $120^{\circ}, 6.10(20)$ & $60.96(24)$ & 290 & 40 \\
\hline $120^{\circ}, 6.10(20)$ & $121.92(48)$ & 81 & 42 \\
\hline $120^{\circ}, 12.19(40)$ & $2.54(1)$ & 18 & 2 \\
\hline $120^{\circ}, 12.19(40)$ & $30.48(12)$ & 15 & 1.4 \\
\hline $120^{\circ}, 12.19(40)$ & $60.96(24)$ & 140 & 28 \\
\hline $120^{\circ}, 12.19(40)$ & $121.92(48)$ & 380 & 73 \\
\hline $120^{\circ}, 18.29(60)$ & $2.54(1)$ & 60 & 8.4 \\
\hline $120^{\circ}, 18.29(60)$ & $30.48(12)$ & 27 & 3.6 \\
\hline $120^{\circ}, 18.29(60)$ & $60.96(24)$ & 290 & 160 \\
\hline $120^{\circ}, 18.29(60)$ & $121.92(48)$ & 290 & 61 \\
\hline $150^{\circ}, 6.10(20)$ & $2.54(1)$ & 28 & 2.3 \\
\hline $150^{\circ}, 6.10(20)$ & $30.48(12)$ & 3.6 & 0.6 \\
\hline $150^{\circ}, 12.19(40)$ & $2.54(1)$ & 37 & 5.4 \\
\hline $150^{\circ}, 12.19(40)$ & $30.48(12)$ & 21 & 3.1 \\
\hline $210^{\circ}, 6.10(20)$ & $2.54(1)$ & 170 & 7.1 \\
\hline $210^{\circ}, 6.10(20)$ & $30.48(12)$ & 220 & 48 \\
\hline $210^{\circ}, 12.19(40)$ & $2.54(1)$ & 11 & 0.46 \\
\hline $210^{\circ}, 12.19(40)$ & $30.48(12)$ & ND & $\mathrm{ND}$ \\
\hline $240^{\circ}, 6.10(20)$ & $2.54(1)$ & 1,100 & 82 \\
\hline $240^{\circ}, 6.10(20)$ & $30.48(12)$ & 4,700 & 1,400 \\
\hline $240^{\circ}, 12.19(40)$ & $2.54(1)$ & 60 & 7.6 \\
\hline $240^{\circ}, 12.19(40)$ & $30.48(12)$ & 16 & 2 \\
\hline $270^{\circ}, 6.10(20)$ & $2.54(1)$ & 16 & 5.3 \\
\hline $270^{\circ}, 6.10(20)$ & $30.48(12)$ & 53 & 17 \\
\hline $270^{\circ}, 12.19(40)$ & $2.54(1)$ & 22 & 0.93 \\
\hline $270^{\circ}, 12.19(40)$ & $30.48(12)$ & ND & ND \\
\hline $300^{\circ}, 0.91(3)$ & $2.54(1)$ & 230 & 100 \\
\hline $300^{\circ}, 0.91(3)$ & $30.48(12)$ & 780 & 400 \\
\hline
\end{tabular}


Table 2-2

Tritium in Rulison Soil Moisture - July 1972

(Page 5 of 9)

\begin{tabular}{|c|c|c|c|}
\hline Grid Coordinates" & $\begin{array}{c}\text { Sampling Depth } \\
\text { centimeters (inches) }\end{array}$ & $\mathrm{pCi} / \mathrm{mi}^{\mathrm{c}}$ & $\mathrm{pCi} / \mathrm{g}^{\mathrm{d}}$ (soil) \\
\hline $300^{\circ}, 0.91(3)$ & $60.96(24)$ & 3,300 & 1,900 \\
\hline $300^{\circ}, 0.91(3)$ & $91.44(36)$ & 3,800 & 820 \\
\hline $300^{\circ}, 0.91(3)$ & $121.92(48)$ & 5,700 & 1,300 \\
\hline $300^{\circ}, 0.91(3)$ & $152.40(60)$ & 6,400 & 3,200 \\
\hline $300^{\circ}, 0.91(3)$ & $182.88(72)$ & 4,400 & 2,500 \\
\hline $300^{\circ}, 0.91(3)$ & $213.36(84)$ & 2,900 & 1,400 \\
\hline $300^{\circ}, 0.91(3)$ & $243.84(96)$ & 2,700 & 1,300 \\
\hline $300^{\circ}, 6.10(20)$ & $2.54(1)$ & 400 & 115 \\
\hline $300^{\circ}, 6.10(20)$ & $30.48(12)$ & 450 & 150 \\
\hline $300^{\circ}, 6.10(20)$ & $60.96(24)$ & 3,900 & 520 \\
\hline $300^{\circ}, 6.10(20)$ & $121.92(48)$ & 2,500 & 520 \\
\hline $300^{\circ}, 12.19(40)$ & $2.54(1)$ & 42 & 2.1 \\
\hline $300^{\circ}, 12.19(40)$ & $30.48(12)$ & 8.6 & 1.2 \\
\hline $330^{\circ}, 6.10(20)$ & $2.54(1)$ & 350 & 100 \\
\hline $330^{\circ}, 6.10(20)$ & $30.48(12)$ & 600 & 180 \\
\hline $330^{\circ}, 12.19(40)$ & $2.54(1)$ & 65 & 4.1 \\
\hline $330^{\circ}, 12.19(40)$ & $30.48(12)$ & 17 & 1.5 \\
\hline & & & \\
\hline$S-1, E-1$ & $2.54(1)$ & 6.5 & 0.04 \\
\hline S-1, E-1 & $30.48(12)$ & ND & ND \\
\hline $\mathrm{S}-1, \mathrm{E}-2$ & $2.54(1)$ & 10 & 0.006 \\
\hline $\mathrm{S}-1, \mathrm{E}-2$ & $30.48(12)$ & ND & ND \\
\hline$S-1, E-3$ & $2.54(1)$ & $\mathrm{ND}$ & ND \\
\hline $\mathrm{S}-1, \mathrm{E}-3$ & $30.48(12)$ & ND & ND \\
\hline S-2, W-.7 & $2.54(1)$ & ND & ND \\
\hline$S-2, W-.7$ & $30.48(12)$ & 7.3 & 1.2 \\
\hline S-3, E-0 & $2.54(1)$ & 1,500 & 810 \\
\hline S-3, E-0 & $30.48(12)$ & 66 & 11 \\
\hline S-3, E-2 & $2.54(1)$ & $\mathrm{ND}$ & ND \\
\hline S-3, E-2 & $30.48(12)$ & $\mathrm{ND}$ & ND \\
\hline S-3.8, E-1.4 & $2.54(1)$ & $\mathrm{ND}$ & ND \\
\hline S-3.8, E-1.4 & $30.48(12)$ & ND & ND \\
\hline S-5, E-0 & $2.54(1)$ & $\mathrm{ND}$ & ND \\
\hline S-5, E-0 & $30.48(12)$ & ND & ND \\
\hline S-5, E-2 & $2.54(1)$ & ND & ND \\
\hline S-5, E-2 & $30.48(12)$ & $\mathrm{ND}$ & ND \\
\hline S-5, E-4 & $2.54(1)$ & ND & ND \\
\hline S-5, E-4 & $30.48(12)$ & 20 & 3.4 \\
\hline S-5.7,W-2 & $2.54(1)$ & 200 & 7.5 \\
\hline S-5.7,W-2 & $30.48(12)$ & 2.9 & 0.56 \\
\hline S-7, E-0 & $2.54(1)$ & ND & $\mathrm{ND}$ \\
\hline S-7, E-0 & $30.48(12)$ & ND & ND \\
\hline S-7, E-2 & $2.54(1)$ & ND & $\mathrm{ND}$ \\
\hline S-7, E-2 & $30.48(12)$ & ND & ND \\
\hline$S-7, E-4$ & $2.54(1)$ & ND & ND \\
\hline S-7, E-4 & $30.48(12)$ & ND & ND \\
\hline S-7, E-6 & $2.54(1)$ & 70 & 29 \\
\hline S-7, E-6 & $30.48(12)$ & ND & ND \\
\hline
\end{tabular}

Refer to footnotes at end of table. 
Table 2-2

Tritium in Rulison Soil Moisture - July 1972

(Page 6 of 9)

\begin{tabular}{|c|c|c|c|}
\hline Grid Coordinates $^{a}$ & $\begin{array}{l}\text { Sampling Depth } \\
\text { centimeters (inches) }\end{array}$ & $\mathrm{pCi} / \mathrm{ml}^{\mathrm{c}}$ & $\mathrm{pCi} / \mathrm{g}^{d}$ (soil) \\
\hline S-7,E-8 & $2.54(1)$ & 770 & 13 \\
\hline S-7, E-8 & $30.48(12)$ & ND & $\mathrm{ND}$ \\
\hline S-7, E-10 & $2.54(1)$ & 91 & 2.3 \\
\hline S-7, E-10 & $30.48(12)$ & $\mathrm{ND}$ & $\mathrm{ND}$ \\
\hline$S-7, E-12$ & $2.54(1)$ & 6.7 & 0.17 \\
\hline$S-7, E-12$ & $30.48(12)$ & ND & $\mathrm{ND}$ \\
\hline S-7.5,W-2.7 & $2.54(1)$ & 43 & 0.37 \\
\hline$S-7.5, W-2.7$ & $30.48(12)$ & ND & $\mathrm{ND}$ \\
\hline$S-8, W-1.5$ & $2.54(1)$ & $\mathrm{ND}$ & $\mathrm{ND}$ \\
\hline$S-8, W-1.5$ & $30.48(12)$ & ND & $\mathrm{ND}$ \\
\hline S-9, E-0 & $2.54(1)$ & ND & $\mathrm{ND}$ \\
\hline S-9, E-0 & $30.48(12)$ & ND & ND \\
\hline S-9, E-2 & $2.54(1)$ & 100 & 41 \\
\hline S-9, E-2 & $30.48(12)$ & ND & $\mathrm{ND}$ \\
\hline S-9, E-4 & $2.54(1)$ & $\mathrm{ND}$ & ND \\
\hline S-9, E-4 & $30.48(12)$ & ND & $\mathrm{ND}$ \\
\hline S-9, E-6 & $2.54(1)$ & $\mathrm{ND}$ & ND \\
\hline S-9, E-6 & $30.48(12)$ & ND & ND \\
\hline S-9, E-8 & $2.54(1)$ & ND & $\mathrm{ND}$ \\
\hline$S-9, E-8$ & $30.48(12)$ & ND & $\mathrm{ND}$ \\
\hline S-9, E-10 & $2.54(1)$ & 130 & 4.5 \\
\hline S-9, E-10 & $30.48(12)$ & $\mathrm{ND}$ & $\mathrm{ND}$ \\
\hline S-9, E-12 & $2.54(1)$ & 110 & 2.1 \\
\hline S-9, E-12 & $30.48(12)$ & $\mathrm{ND}$ & $\mathrm{ND}$ \\
\hline S-9.4, W-3.4 & $2.54(1)$ & 3,900 & 32 \\
\hline S-9.4,W-3.4 & $30.48(12)$ & 230 & 25 \\
\hline$S-10, W-1.5$ & $2.54(1)$ & ND & $\mathrm{ND}$ \\
\hline$S-10, W-1.5$ & $30.48(12)$ & $\mathrm{ND}$ & $\mathrm{ND}$ \\
\hline$S-10.3, E-10.1$ & $2.54(1)$ & $\mathrm{ND}$ & $\mathrm{ND}$ \\
\hline S-10.3, E-10.1 & $30.48(12)$ & ND & $\mathrm{ND}$ \\
\hline S-10.3, E-10.1 & $60.96(24)$ & ND & $\mathrm{ND}$ \\
\hline S-10.3, E-10.1 & $121.92(48)$ & ND & $\mathrm{ND}$ \\
\hline S-11, E-0 & $2.54(1)$ & 610 & 11 \\
\hline$S-11, E-0$ & $30.48(12)$ & ND & $\mathrm{ND}$ \\
\hline S-11, E-2 & $2.54(1)$ & ND & $\mathrm{ND}$ \\
\hline$S-11, E-2$ & $30.48(12)$ & ND & $\mathrm{ND}$ \\
\hline S-11, E-4 & $2.54(1)$ & 62 & 0.98 \\
\hline S-11, E-4 & $30.48(12)$ & $\mathrm{ND}$ & $\mathrm{ND}$ \\
\hline$S-11, E-6$ & $2.54(1)$ & ND & $\mathrm{ND}$ \\
\hline S-11, E-6 & $30.48(12)$ & ND & $\mathrm{ND}$ \\
\hline$S-11, E-8$ & $2.54(1)$ & ND & $\mathrm{ND}$ \\
\hline S-11, E-8 & $30.48(12)$ & ND & $\mathrm{ND}$ \\
\hline S-11, E-10 & $2.54(1)$ & $\mathrm{ND}$ & $\mathrm{ND}$ \\
\hline S-11, E-10 & $30.48(12)$ & $\mathrm{ND}$ & $\mathrm{ND}$ \\
\hline S-11, E-12 & $2.54(1)$ & ND & $\mathrm{ND}$ \\
\hline $\mathrm{S}-11, \mathrm{E}-12$ & $30.48(12)$ & ND & $\mathrm{ND}$ \\
\hline S-11, E-14 & 2.54 (1) & $\mathrm{ND}$ & $\mathrm{ND}$ \\
\hline$S-11, E-14$ & $30.48(12)$ & ND & $\mathrm{ND}$ \\
\hline S-11.2, W-4 & $2.54(1)$ & 280 & 21 \\
\hline S-11.2, W-4 & $30.48(12)$ & ND & $\mathrm{ND}$ \\
\hline S-11.7,E-3.11 & 2.54 (1) & $\mathrm{ND}$ & $\mathrm{ND}$ \\
\hline
\end{tabular}

Refer to footnotes at end of table. 
Table 2-2

Tritium in Rulison Soil Moisture - July 1972

(Page 7 of 9)

\begin{tabular}{|c|c|c|c|}
\hline Grid Coordinates" & $\begin{array}{c}\text { Sampling Depth } \\
\text { centimeters (inches) }\end{array}$ & $\mathrm{pCi} / \mathrm{ml}^{\mathrm{c}}$ & pCi/g (soil) \\
\hline S-11.7,E-3.1 & $30.48(12)$ & ND & $\mathrm{ND}$ \\
\hline S-11.7, E-8.7 & $2.54(1)$ & ND & $\mathrm{ND}$ \\
\hline S-11.7,E-8.7 & $30.48(12)$ & ND & $\mathrm{ND}$ \\
\hline S-11.7, E-8.7 & $60.96(24)$ & ND & $\mathrm{ND}$ \\
\hline S-11.7, E-8.7 & $121.92(48)$ & ND & ND \\
\hline S-12, E-1 & $2.54(1)$ & $\mathrm{ND}$ & $\mathrm{ND}$ \\
\hline $\mathrm{S}-12, \mathrm{E}-1$ & $30.48(12)$ & ND & $\mathrm{ND}$ \\
\hline S-12, E-5 & $2.54(1)$ & 18 & 0.2 \\
\hline S-12, E-5 & $30.48(12)$ & ND & $\overline{N D}$ \\
\hline$S-12, W-1.5$ & $2.54(1)$ & 3.8 & 0.2 \\
\hline$S-12, W-1.5$ & $30.48(12)$ & ND & ND \\
\hline S-12.4, E-3.8 & $2.54(1)$ & ND & ND \\
\hline S-12.4,E-3.8 & $30.48(12)$ & $\mathrm{ND}$ & $\mathrm{ND}$ \\
\hline S-12.8,E-1.9 & $2.54(1)$ & ND & $\mathrm{ND}$ \\
\hline S-12.8, E-1.9 & $30.48(12)$ & ND & $\mathrm{ND}$ \\
\hline S-12.8, E-1.9 & $60.96(24)$ & ND & ND \\
\hline S-12.8, E-1.9 & $121.92(48)$ & ND & $\mathrm{ND}$ \\
\hline $\mathrm{S}-13, \mathrm{E}-0$ & $2.54(1)$ & ND & ND \\
\hline S-13, E-0 & $30.48(12)$ & ND & ND \\
\hline S-13,E-6 & $2.54(1)$ & $\mathrm{ND}$ & ND \\
\hline $\mathrm{S}-13, \mathrm{E}-6$ & $30.48(12)$ & ND & $\mathrm{ND}$ \\
\hline$S-13, E-8$ & $2.54(1)$ & 14 & 0.32 \\
\hline S-13, E-8 & $30.48(12)$ & 84 & 6.3 \\
\hline S-13, E-10 & $2.54(1)$ & 1 & 0.01 \\
\hline$S-13, E-10$ & $30.48(12)$ & ND & $\mathrm{ND}$ \\
\hline$S-13, E-12$ & $2.54(1)$ & $\mathrm{ND}$ & $\mathrm{ND}$ \\
\hline$S-13, E-12$ & $30.48(12)$ & ND & ND \\
\hline S-13, E-14 & $2.54(1)$ & ND & $\overline{N D}$ \\
\hline S-13, E-14 & $30.48(12)$ & ND & $\mathrm{ND}$ \\
\hline S-13.1,E-7.3 & $2.54(1)$ & ND & ND \\
\hline S-13.1,E-7.3 & $30.48(12)$ & $\mathrm{ND}$ & $\mathrm{ND}$ \\
\hline S-13.1, E-7.3 & $60.96(24)$ & $\mathrm{ND}$ & ND \\
\hline S-13.1, E-7.3 & $121.92(48)$ & $\mathrm{ND}$ & $\mathrm{ND}$ \\
\hline$S-13.1, W-4.8$ & $2.54(1)$ & 690 & 290 \\
\hline S-13.1,W-4.8 & $30.48(12)$ & ND & $\mathrm{ND}$ \\
\hline S-13.2, E-4.5 & $2.54(1)$ & $\mathrm{ND}$ & $\mathrm{ND}$ \\
\hline S-13.2, E-4.5 & $30.48(12)$ & ND & ND \\
\hline S-13.5, E-2.8 & $2.54(1)$ & ND & $\mathrm{ND}$ \\
\hline$S-13.5, E-2.8$ & $30.48(12)$ & $\mathrm{ND}$ & ND \\
\hline S-13.9, E-5.2 & $2.54(1)$ & ND & $\mathrm{ND}$ \\
\hline S-13.9, E-5.2 & $30.48(12)$ & ND & $\mathrm{ND}$ \\
\hline S-14,E- 8 & $2.54(1)$ & ND & $\mathrm{ND}$ \\
\hline S-14, E-.8 & $30.48(12)$ & ND & $\mathrm{ND}$ \\
\hline$S-14, W-3.4$ & $2.54(1)$ & 120 & 1.1 \\
\hline S-14,W-3.4 & $30.48(12)$ & 45 & 8.5 \\
\hline S-14.2, E-3.4 & $2.54(1)$ & ND & ND \\
\hline S-14.2, E-3.4 & $30.48(12)$ & 12 & 1.6 \\
\hline S-14.2, E-3.4 & $60.96(24)$ & ND & $\mathrm{ND}$ \\
\hline S-14.2, E-3.4 & $121.92(48)$ & ND & $\mathrm{ND}$ \\
\hline S-14.6, E-5.9 & $2.54(1)$ & 20 & 0.1 \\
\hline S-14.6,E-5.9 & $30.48(12)$ & ND & ND \\
\hline
\end{tabular}

Refer to footnotes at end of table. 
Table 2-2

Tritium in Rulison Soil Moisture - July 1972

(Page 8 of 9)

\begin{tabular}{|c|c|c|c|}
\hline Grid Coordinates" & $\begin{array}{l}\text { Sampling Depth } \\
\text { centimeters (inches) }\end{array}$ & $\mathrm{pCi} / \mathrm{ml}^{\mathrm{c}}$ & $\mathrm{pCi} / \mathrm{g}^{\mathrm{d}}$ (soil) \\
\hline S-14.6, E-5.9 & $60.96(24)$ & $\mathrm{ND}$ & $\overline{\mathrm{ND}}$ \\
\hline S-14.6, E-5.9 & $121.92(48)$ & ND & ND \\
\hline $\mathrm{S}-14.7, \mathrm{E}-1.6$ & $2.54(1)$ & ND & ND \\
\hline S-14.7,E-1.6 & $30.48(12)$ & ND & $\mathrm{ND}$ \\
\hline S-15, E-12 & $2.54(1)$ & 26 & 0.03 \\
\hline S-15, E-12 & $30.48(12)$ & $\mathrm{ND}$ & $\mathrm{ND}$ \\
\hline S-15, E-14 & $2.54(1)$ & $\mathrm{ND}$ & $\mathrm{ND}$ \\
\hline S-15, E-14 & $30.48(12)$ & $\mathrm{ND}$ & $\mathrm{ND}$ \\
\hline $\mathrm{S}=15, \mathrm{~W}-1.8$ & $2.54(1)$ & 76 & 1.4 \\
\hline$S-15, W-1.8$ & $30.48(12)$ & 1,400 & 210 \\
\hline S-15.4, E-2.2 & $2.54(1)$ & $\mathrm{ND}$ & $\mathrm{ND}$ \\
\hline $\mathrm{S}-15.4, \mathrm{E}-2.2$ & $30.48(12)$ & $\mathrm{ND}$ & $\mathrm{ND}$ \\
\hline S-15.4, E-5 & $2.54(1)$ & 55 & 0.4 \\
\hline S-15.4, E-5 & $30.48(12)$ & $\mathrm{ND}$ & $\mathrm{ND}$ \\
\hline S-15.4, E-5 & $60.96(24)$ & ND & $\mathrm{ND}$ \\
\hline S-15.4, E-5 & $121.92(48)$ & ND & $\mathrm{ND}$ \\
\hline S-15.4,E-6.6 & $2.54(1)$ & 480 & 7.6 \\
\hline $\mathrm{S}-15.4, \mathrm{E}-6.6$ & $30.48(12)$ & ND & $\mathrm{ND}$ \\
\hline S-15.4, E-8 & $2.54(1)$ & ND & $\mathrm{ND}$ \\
\hline S-15.4, E-8 & $30.48(12)$ & 12 & 2.1 \\
\hline S-16, E-0 & $2.54(1)$ & ND & ND \\
\hline S-16, E-O & $30.48(12)$ & $\mathrm{ND}$ & ND \\
\hline S-16.1, E-3 & $2.54(1)$ & 520 & 16 \\
\hline S-16.1, E-3 & $30.48(12)$ & ND & ND \\
\hline S-16.2, E-4 & $2.54(1)$ & 48 & 1.3 \\
\hline S-16.2, E-4 & $30.48(12)$ & ND & ND \\
\hline S-16.2, E-4 & $60.96(24)$ & $\mathrm{ND}$ & ND \\
\hline S-16.2, E-4 & $121.92(48)$ & ND & ND \\
\hline S-16.6, E-6.6 & $2.54(1)$ & 8.5 & 0.2 \\
\hline S-16.6, E-6.6 & $30.48(12)$ & 5.3 & 0.5 \\
\hline S-16.6, E-6.6 & $60.96(24)$ & 8.9 & 0.9 \\
\hline S-16.6, E-6.6 & $121.92(48)$ & $\mathrm{ND}$ & $\mathrm{ND}$ \\
\hline S-16.6, E-8 & $2.54(1)$ & 5.9 & 0.24 \\
\hline S-16.6, E-8 & $30.48(12)$ & 5.7 & 0.3 \\
\hline S-17, E-10 & $2.54(1)$ & ND & ND \\
\hline S-17, E-10 & $30.48(12)$ & ND & $\mathrm{ND}$ \\
\hline S-17, E-12 & $2.54(1)$ & ND & ND \\
\hline S-17, E-12 & $30.48(12)$ & 46 & 2.4 \\
\hline S-17, E-14 & $2.54(1)$ & 190 & 54 \\
\hline $\mathrm{S}-17, \mathrm{E}-14$ & $30.48(12)$ & ND & ND \\
\hline S-17.1, E-1.8 & $2.54(1)$ & 89 & 1.2 \\
\hline S-17.1, E-1.8 & $30.48(12)$ & ND & ND \\
\hline $\mathrm{S}-18, \mathrm{E}-6.5$ & $2.54(1)$ & 11 & 0.1 \\
\hline S-18, E-6.5 & $30.48(12)$ & 14 & 1.3 \\
\hline S-18.2, E-3.5 & $2.54(1)$ & 270 & 56 \\
\hline S-18.2, E-3.5 & $30.48(12)$ & ND & ND \\
\hline S-19, E-8 & $2.54(1)$ & 11 & 0.67 \\
\hline S-19, E-8 & $30.48(12)$ & ND & $\mathrm{ND}$ \\
\hline S-19, E-9 & $2.54(1)$ & ND & ND \\
\hline S-19, E-9 & $30.48(12)$ & ND & ND \\
\hline S-19, E-9 & $60.96(24)$ & $\mathrm{ND}$ & ND \\
\hline
\end{tabular}

Refer to footnotes at end of table. 
Table 2-2

Tritium in Rulison Soil Moisture - July 1972

(Page 9 of 9)

\begin{tabular}{|c|c|c|c|}
\hline Grid Coordinates & $\begin{array}{l}\text { Sampling Depth } \\
\text { centimeters (inches) }\end{array}$ & $\mathrm{pCV} / \mathbf{m l}^{\mathrm{c}}$ & pCi/g (soil) \\
\hline S-19, E-9 & $121.92(48)$ & ND & ND \\
\hline S-19, E-10 & $2.54(1)$ & ND & ND \\
\hline S-19, E-10 & $30.48(12)$ & 50 & 4.9 \\
\hline S-19, E-12 & $2.54(1)$ & ND & ND \\
\hline S-19, E-12 & $30.48(12)$ & 5.8 & 0.28 \\
\hline S-19, E-14 & $2.54(1)$ & ND & $\mathrm{ND}$ \\
\hline S-19, E-14 & $30.48(12)$ & ND & ND \\
\hline S-19.3, E-5.1 & $2.54(1)$ & 58 & 1.4 \\
\hline S-19.3, E-5.1 & $30.48(12)$ & 11 & 2.3 \\
\hline S-20, E-12 & $2.54(1)$ & 12 & 0.56 \\
\hline S-20, E-12 & $30.48(12)$ & 15 & 0.39 \\
\hline S-20.4, E-6.8 & $2.54(1)$ & ND & ND \\
\hline S-20.4, E-6.8 & $30.48(12)$ & ND & ND \\
\hline S-21, E-14 & $2.54(1)$ & 19 & 0.43 \\
\hline S-21, E-14 & $30.48(12)$ & ND & ND \\
\hline S-21.5, E-8.5 & $2.54(1)$ & 300 & 2.2 \\
\hline S-21.5, E-8.5 & $30.48(12)$ & ND & ND \\
\hline S-22.5, E-10.2 & $2.54(1)$ & 130 & 0.52 \\
\hline S-22.5, E-10.2 & $30.48(12)$ & ND & ND \\
\hline $\mathrm{S}-23.2, \mathrm{E}-17$ & $2.54(1)$ & 9.4 & 4.1 \\
\hline S-23.2, E-17 & $30.48(12)$ & ND & ND \\
\hline S-23.5, E-12 & $2.54(1)$ & 13 & 0.54 \\
\hline S-23.5, E-12 & $30.48(12)$ & 4.1 & 0.71 \\
\hline S-23.5, E-12 & $2.54(1)$ & 32 & 1.2 \\
\hline S-23.8, E-15.3 & $30.48(12)$ & ND & ND \\
\hline S-24.6, E-13.7 & $2.54(1)$ & 47,000 & 20,000 \\
\hline S-24.6, E-13.7 & $30.48(12)$ & 860 & 140 \\
\hline S-24.6, E-17 & $2.54(1)$ & 36 & 0.39 \\
\hline $\mathrm{S}-24.6, \mathrm{E}-17$ & $30.48(12)$ & 19 & 3.2 \\
\hline S-25.4, E-15.4 & $2.54(1)$ & 1,400 & 22 \\
\hline S-25.4, E-15.4 & $30.48(12)$ & 1,700 & 235 \\
\hline
\end{tabular}

Source: Eberline, 1977

a Cardinal coordinates referenced to entrance gate post scale; 1 unit equals 3.05 meters ( 10 feet).

Radial coordinates are in degrees and meters (feet) referenced to flare stack.

'Sampling depth increments are $2.54 \mathrm{~cm}$ (i.e., $2.54 \mathrm{~cm}$ is from 0 to $2.54 \mathrm{~cm}, 30.48$ is from 27.94 to $30.48 \mathrm{~cm}$, etc.)

(1" [i.e., 1" is from 0 to $1^{\prime \prime}, 12^{\prime \prime}$ is from $11^{\prime \prime}$ to $12^{\prime \prime}$, etc.]).

'Picocurie per milliliter

'Picocurie per gram 
Table 2-3

Tritium in Vegetation - July 1972

\begin{tabular}{|c|c|c|c|c|c|c|}
\hline \multirow[b]{2}{*}{$\begin{array}{l}\text { Grid Coordinates } \\
\text { degrees, meters (feet) }\end{array}$} & \multirow[b]{2}{*}{$\begin{array}{c}\text { Dry/Wet } \\
\text { Ratio }\end{array}$} & \multicolumn{2}{|c|}{ Unbound $^{b}$} & \multicolumn{2}{|c|}{ Bound $^{c}$} & \multirow{2}{*}{$\begin{array}{c}\text { Total } \\
\mathrm{pCi} / \mathrm{g} \text { (wet) }\end{array}$} \\
\hline & & $\mathrm{pCi} / \mathrm{ml}^{\mathrm{d}}(\mathrm{H} 2 \mathrm{O})^{\mathrm{e}}$ & $\mathrm{pci} / \mathrm{g}^{\prime}$ (wet) & $\begin{array}{c}\mathrm{pCi} / \mathrm{ml}\left(\mathrm{H}_{2} \mathrm{O}\right) \\
\text { (water from } \\
\text { oxidation) } \\
\end{array}$ & $\mathrm{pCi} / \mathrm{g}$ (wet) & \\
\hline $000^{\circ}, 152.40(500)$ & 0.38 & 7 & 4.3 & $<31$ & $<1.7$ & $\approx 4.3$ \\
\hline $000^{\circ}, 304.80(1,000)$ & 0.42 & 7.2 & 2.8 & $<8.3$ & $<1.4$ & $\approx 2.8$ \\
\hline $090^{\circ}, 152.40(500)$ & 0.23 & 4.5 & 3.5 & $<32$ & $<1.5$ & $\approx 3.5$ \\
\hline $090^{\circ}, 304.80(1,000)$ & 0.30 & 8.1 & 5.7 & $<33$ & $<1.1$ & $\approx 5.7$ \\
\hline $180^{\circ}, 152.40(500)$ & 0.22 & 75 & 58 & $<16$ & $<0.9$ & $\approx 58$ \\
\hline $180^{\circ}, 304.80(1,000)$ & 0.25 & 7.1 & 5.3 & $<11$ & $<0.8$ & $\approx 5.3$ \\
\hline $270^{\circ}, 152.40(500)$ & 0.19 & 5.5 & 4.5 & $<28$ & $<0.8$ & $\approx 4.5$ \\
\hline $270^{\circ}, 304.80(1,000)$ & 0.25 & 7.5 & 5.6 & $<14$ & $<1.0$ & $\approx 5.6$ \\
\hline $030^{\circ}, 1.52(5)$ & 0.13 & 170 & 150 & 190 & 5.3 & 160 \\
\hline $120^{\circ}, 12.19(40)$ & 0.27 & 64 & 47 & 97 & 3.6 & 51 \\
\hline${ }^{*} \mathrm{~N}-14, \mathrm{~W}-2$ & 0.22 & 150 & 120 & 41 & 2.3 & 120 \\
\hline
\end{tabular}

Source: Eberline, 1977

${ }^{\star}$ West of tank 3, referenced to entrance gate post.

${ }^{a}$ Coordinates are in degrees and meters (feet) referenced to flare stack.

bunbound; tritium in water that was removable by drying the sample in an electric oven for 16 hours.

'Bound; tritium converted to water form by oxidizing the dried sample.

${ }^{d}$ Picocurie per milliliter

ewater

Picocurie per gram 
A Hydrologic Program Advisory Group reviewed the hydrologic monitoring program proposed for the Rulison Site at a meeting in December 1971. They found the program adequate and recommended its immediate initiation. The U.S. Environmental Protection Agency (EPA), Las Vegas, Nevada, has been conducting the monitoring program since that time (ERDA, 1977, p. 33). Sampling locations are presented on Figure 2-11. Analytical results, to date, are given in Appendix A. Results of the Comprehensive Environmental Response, Compensation, and Liability Act (CERCLA) Preliminary Assessment prepared by Desert Research Institute (DRI) for the Rulison Site in 1988 recommended that the hydrologic monitoring program be continued and periodically updated as new monitoring wells and hydrologic data become available (p. 3.6.21).

Unless otherwise specified, all samples collected for the hydrologic monitoring program are analyzed for tritium. All samples are also analyzed for gross alpha and gross beta radioactivity and are given a gamma spectral scan. Gross chemistry analyses, comparable to the USGS chemical water quality analyses, will be performed on all samples collected on the initial sample run. Based on the results of those analyses, suspect samples will be analyzed for appropriate, naturally occurring, and man-made isotopes. Splits of each collected sample will be retained by EPA for this purpose until it is demonstrated that the need to retain them does not exist. Each water source is sampled once a year, preferably in the early spring, weather permitting.

\subsection{Third Sampling Program}

The third sampling program occurred during September 1 to October 12, 1976, and was associated with the plugging of the emplacement and production wells and abandonment of the Rulison Site. It was designed to consider the history of the site and then to complete all requirements for radiation contamination clearance. It primarily consisted of sampling soil at the following locations:

- At two locations that exceeded the current guideline for tritium in the 1972 cleanup

- At the location of a known spill which occurred during the final cleanup

- In the vicinity of decontamination work

- Around the R-E wellhead location

In addition, the creek was sampled above and below the site, and the same two springs (one on the site, one about $274 \mathrm{~m}$ [300 yards] down the road) were sampled. 


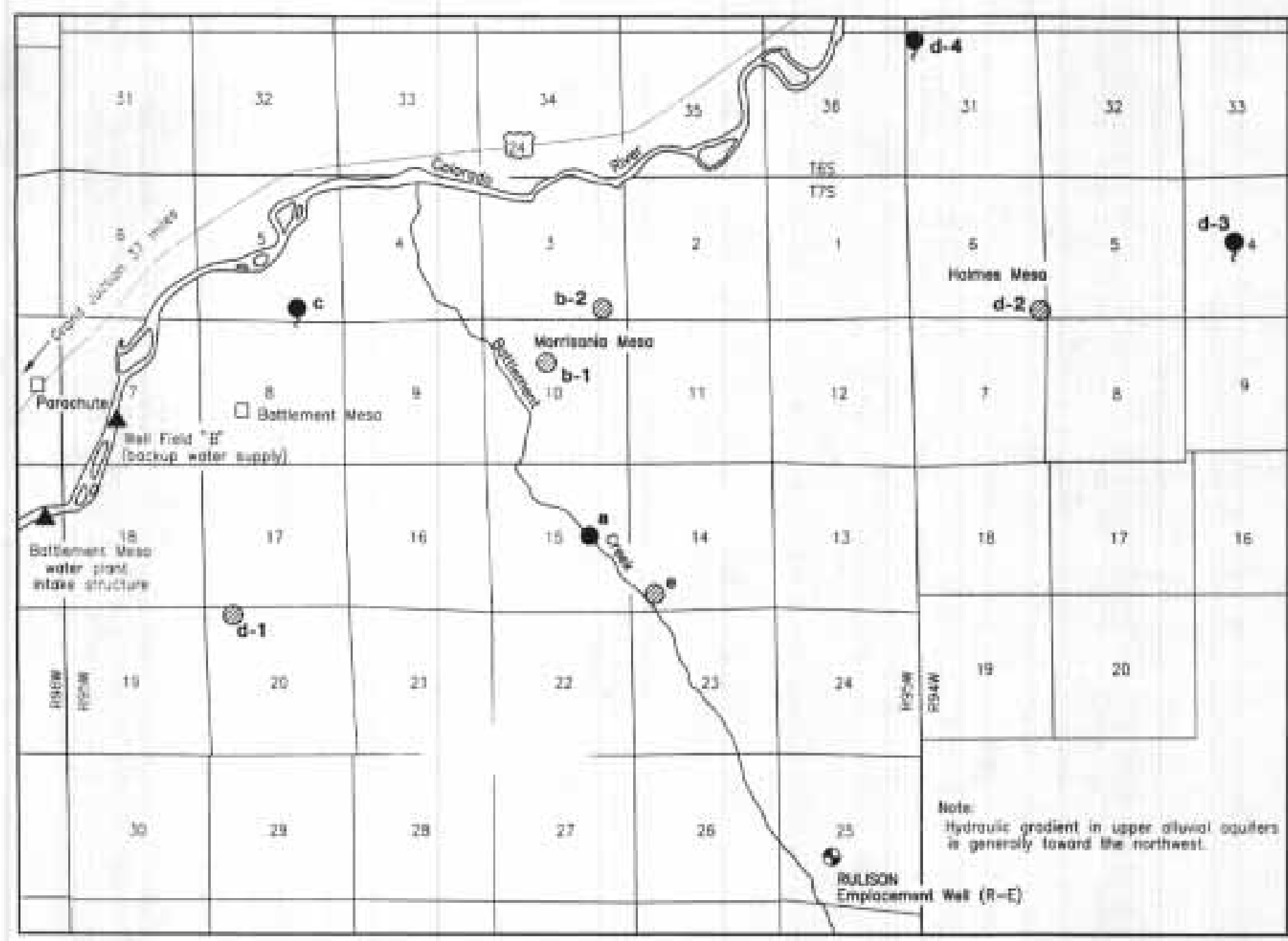

LEFEM

SCASE

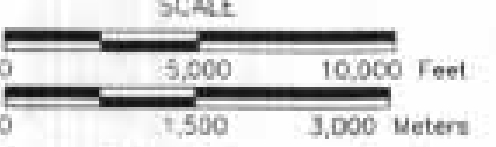

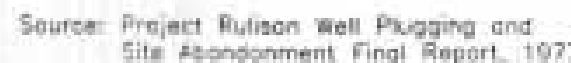

Wat becotion nameiert

9. Spring location sempled

- Creek scotition sompled

Town

Q Wel at surtoce ground zeor

A Manicipal watur tupety hitek

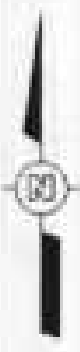

SMPLUG POATS

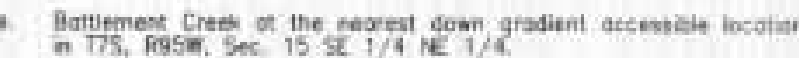

b. Two private wetion in aliumum on Nomsana Hese Leabisns

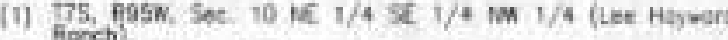

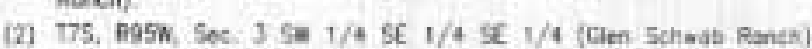

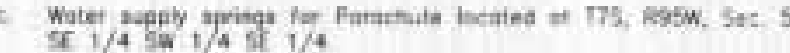

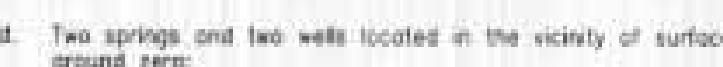

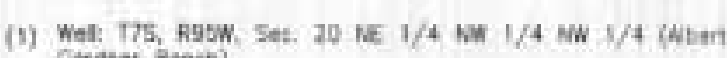

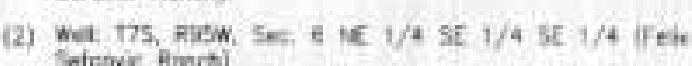

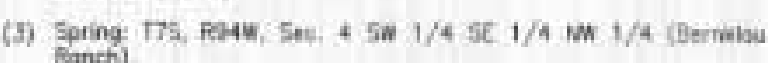

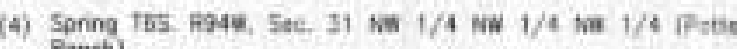

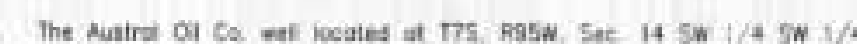

FIGURE $2-11$

PRONECT RULSON, LONG-TERM HYOROLOGIC MONITORING PROGRAM SAMPUNG DATA POINTS, DECEMEER 1971, GARFIELD COUNTY, COLORADO 


\section{Sampling Point N-14.2, E-.7 (Refer to Figure 2-10)}

In July 1972, the samples taken at 61- and 91.4-cm (24- and 36-in.) depths contained concentrations of tritium at 35,000 and 34,000 pCi/ $/ \mathrm{mL}$, respectively, in soil moisture. The guideline was $30,000 \mathrm{pCi} / \mathrm{mL}$ (ERDA, 1976). This contamination was the result of a known spill from a valve that froze and broke during the 1971 to 1972 winter. This sampling point and the area adjacent to the spill were sampled thoroughly. Results of analyses showed that intervening time and weather had reduced contamination to negligible levels. The sample locations and results of analyses are shown on Figure 2-12 and in Table 2-4.

\section{Sampling Point S-24.6, E-13.7 (Refer to Figure 2-9)}

The surface sample taken at this point in July 1972 contained $47,000 \mathrm{pCi} / \mathrm{mL}$ tritium in soil moisture. This was the result of a spill that occurred during production test operations. This point and the adjacent area, including the separator location, were sampled. Results of analyses showed that soil contamination at this location is now negligible. The sample locations and results of analyses are shown on Figure 2-13 and in Table 2-5.

\section{Accidental Spill Area}

On September 1, 1976, the separator was being moved onto the decontamination pan. It was dropped about half onto the pan, and liquid spilled from the separator onto the pan and onto the soil southwest of the pan. An estimated 60 gallons spilled on the soil. The tritium concentration in the separator liquid was about $230,000 \mathrm{pCi} / \mathrm{mL}$. Soil visibly moistened by the liquid was picked up, mixed with diatomaceous earth for additional drying, and was contained in plastic-lined, 55-gallon steel drums. Preliminary samples were taken, and more soil was picked up as indicated. Figure 2-14 shows a sketch of the spill area after 15 drums of soil were removed.

On September 16, 1976, the area was divided into a 1.5-m (5-ft) grid locating 42 sampling points, and a surface sample was taken at each point. Figure 2-15 shows that the contaminated area was delineated and that the decontamination effort had been very effective. All points sampled were less than the guideline; the highest concentration detected was $13,078 \mathrm{pCi} / \mathrm{mL}$ tritium in soil moisture.

On September 21, 1976, five more drums of soil were removed from the area of highest concentration as indicated by the contour boundary line on Figure 2-16. Samples were taken the length and direction of the removed soil as shown also on Figure 2-16. 


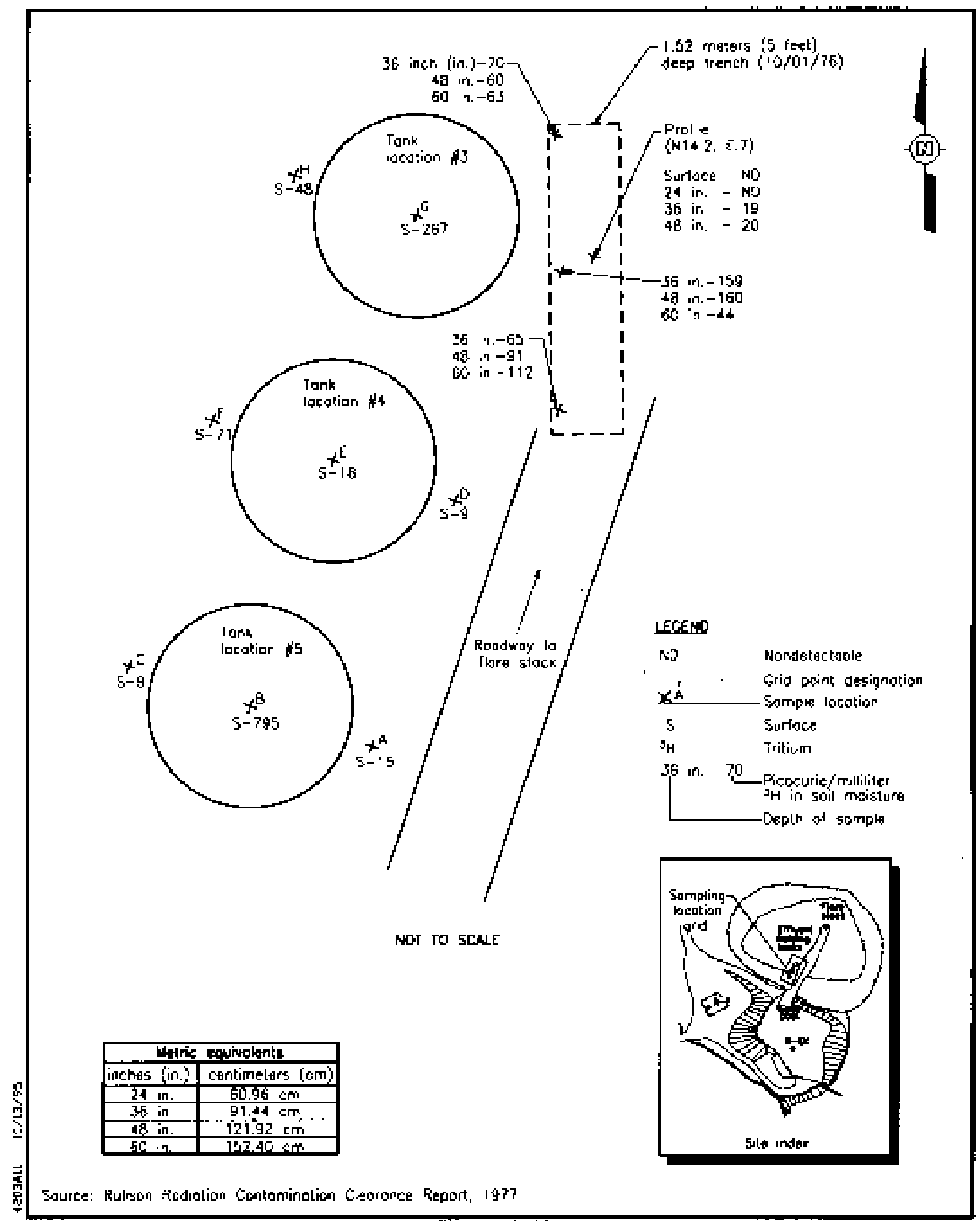

Figure 2-12

Soil Sampling, Fulison Tank Area, October 1976, Garfield County, Colorado 


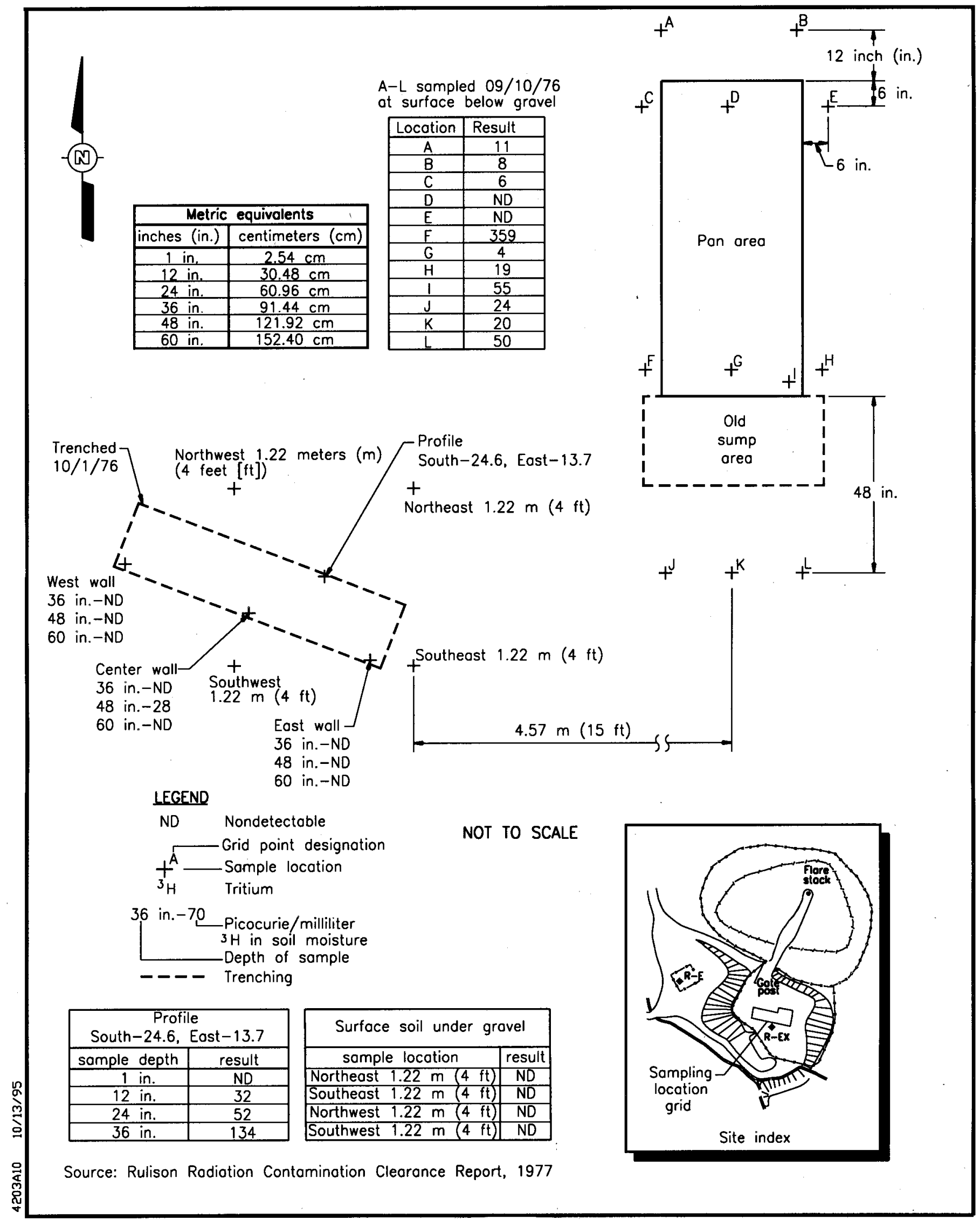

Figure 2-13

Soil Sampling, Rulison Separator Pan Area (previous pipe spill), September 30, 1976, Garfield County, Colorado 


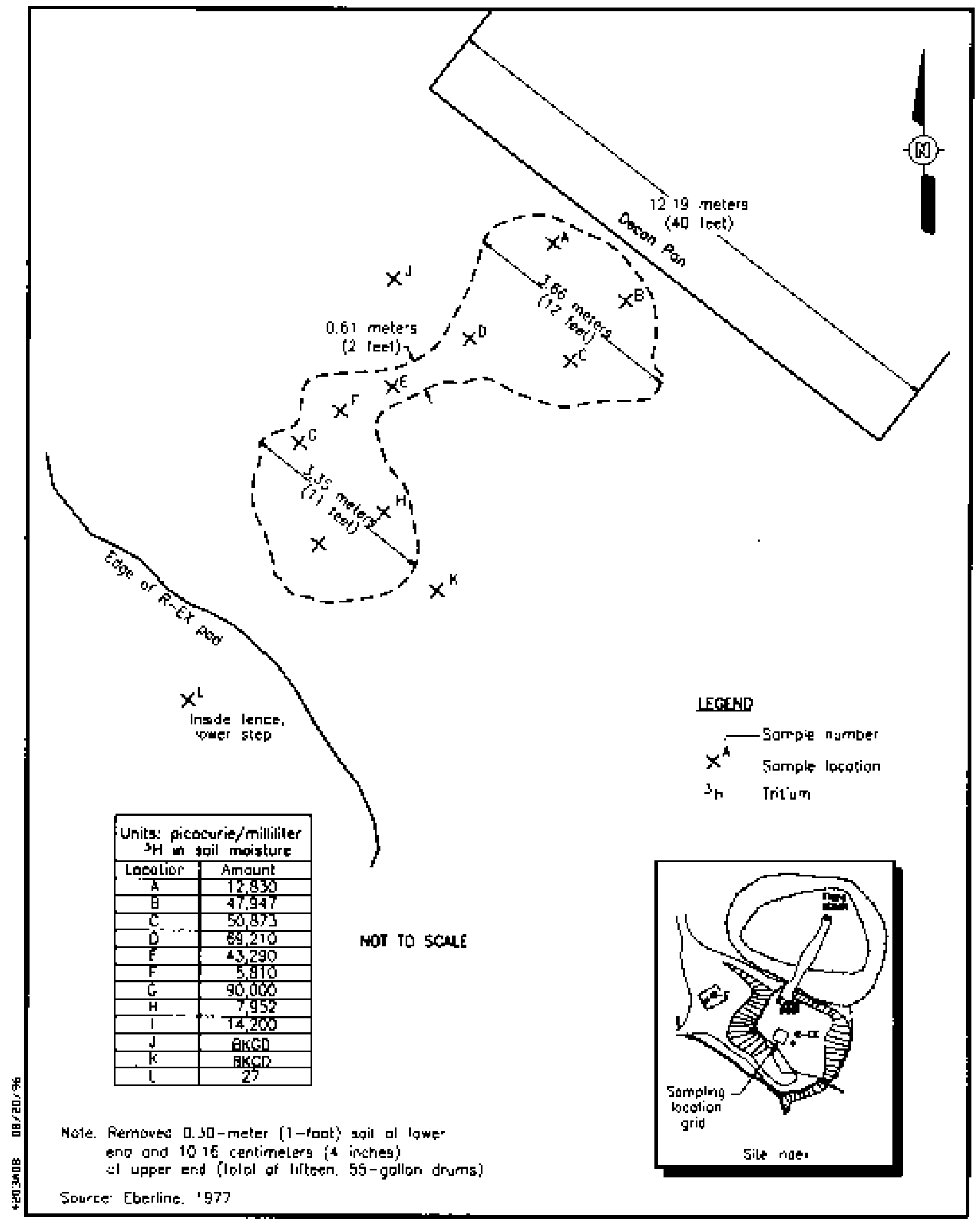

Flgure 2-14

Surface Soil Sampling, Rulison Separator Spill, September 1, 1976, Gerfleld County, Colorado 


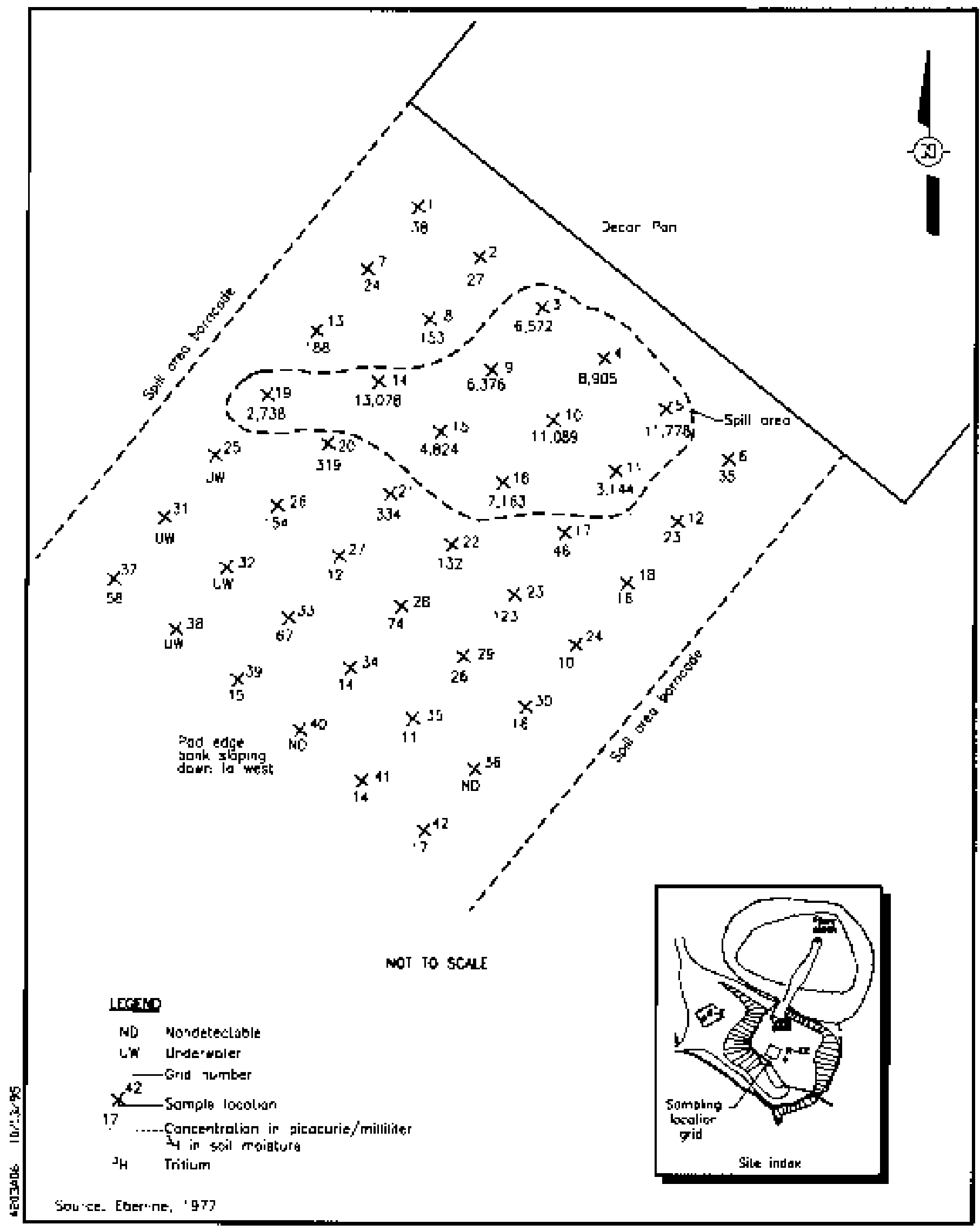

Flgure 2-15

Surface Soil Sampling, Rullson Separator Spill Survey after 15 Drum Soll Removal, September 16, 1976, Garfleld County, Colorado 


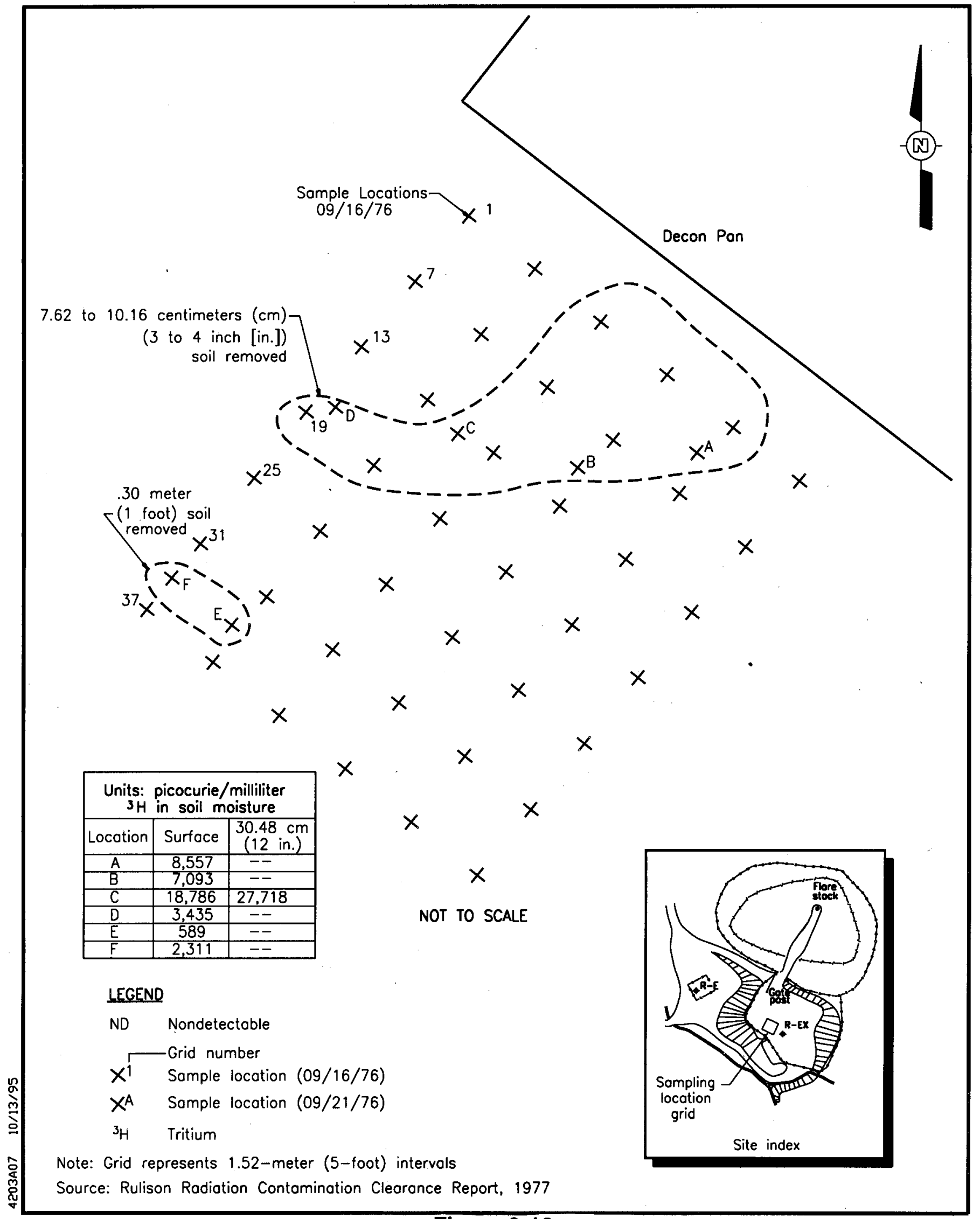

Figure 2-16

Soil Sampling, Rulison Separator Spill after Removal of Five Additional Drums of Soil, September 21, 1976, Garfield County, Colorado 
Table 2-4

Tritium in Soil at Sampling Point N-14.2, E-.7 - October 1976

\begin{tabular}{||l|c|r||}
\hline \multicolumn{1}{|c|}{$\begin{array}{c}\text { Sample } \\
\text { Identification }\end{array}$} & $\begin{array}{c}\text { Sampling Depth } \\
\text { centimeters (inches) }\end{array}$ & $\begin{array}{c}\text { PCi/mI in } \\
\text { Soil Moisture }\end{array}$ \\
\hline \hline Location A & Surface & 75 \\
\hline Location B & Surface & 9 \\
\hline Location C & Surface & 9 \\
\hline Location D & Surface & 18 \\
\hline Location E & Surface & 71 \\
\hline Location F & Surface & 267 \\
\hline Location G & Surface & 48 \\
\hline Location H & Surface & $\mathrm{ND}$ \\
\hline N-14.2, E-.7 & Surface & $\mathrm{ND}$ \\
\hline N-14.2, E-.7 & $60.96(24)$ & 19 \\
\hline N-14.2, E-.7 & $91.44(36)$ & 20 \\
\hline N-14.2, E-.7 & $121.92(48)$ & 65 \\
\hline Trench, South End & $91.44(36)$ & 91 \\
\hline Trench, South End & $121.92(48)$ & 112 \\
\hline Trench, South End & $152.40(60)$ & 159 \\
\hline Trench, Mid-Point & $91.44(36)$ & 160 \\
\hline Trench, Mid-Point & $121.92(48)$ & 44 \\
\hline Trench, Mid-Point & $152.40(60)$ & 70 \\
\hline Trench, North End & $91.44(36)$ & 60 \\
\hline Trench, North End & $121.92(48)$ & 63 \\
\hline Trench, North End & $152.40(60)$ & \\
\hline & & \\
\hline
\end{tabular}

Source: Eberline, 1977

$a_{\text {Picocurie per milliliter }}$ 
Table 2-5

Tritium in Soil at Sampling Point S-24.6, E-13.7 - October 1976

\begin{tabular}{|c|c|c|}
\hline $\begin{array}{c}\text { Sample } \\
\text { Identification } \\
\end{array}$ & $\begin{array}{c}\text { Sampling Depth } \\
\text { centimeters (inches) }\end{array}$ & $\begin{array}{c}\mathrm{pCi} / \mathrm{ml}^{\mathrm{a}} \text { in } \\
\text { Soil Moisture }\end{array}$ \\
\hline Location A & Surface & 11 \\
\hline Location B & Surface & 8 \\
\hline Location C & Surface & 6 \\
\hline Location D & Surface & ND \\
\hline Location $\mathrm{E}$ & Surface & ND \\
\hline Location F & Surface & 359 \\
\hline Location $\mathrm{G}$ & Surface & 4 \\
\hline Location $\mathrm{H}$ & Surface & 19 \\
\hline Location I & Surface & 55 \\
\hline Location J & Surface & 24 \\
\hline Location $\mathrm{K}$ & Surface & 20 \\
\hline Location L & Surface & 50 \\
\hline S-24.6, E-13.7 & $2.54(1)$ & ND \\
\hline S-24.6, E-13.7 & $30.48(12)$ & 32 \\
\hline S-24.6, E-13.7 & $60.96(24)$ & 52 \\
\hline S-24.6, E-13.7 & $91.44(36)$ & 134 \\
\hline $\mathrm{S}-24.6, \mathrm{E}-13.7$ (NE $1.22 \mathrm{~m}[4 \mathrm{ft}])$ & Surface & ND \\
\hline S-24.6, E-13.7 (SE $1.22 \mathrm{~m} \mathrm{[4 \textrm {ft } ] )}$ & Surface & ND \\
\hline S-24.6, E-13.7 (SW $1.22 \mathrm{~m}[4 \mathrm{ft}])$ & Surface & ND \\
\hline S-24.6, E-13.7 (NW $1.22 \mathrm{~m} \mathrm{[4} \mathrm{ft])}$ & Surface & ND \\
\hline Trench Wall, East End & $91.44(36)$ & ND \\
\hline Trench Wall, East End & $121.92(48)$ & ND \\
\hline Trench Wall, East End & $152.40(60)$ & ND \\
\hline Trench Wall, Center & $91.44(36)$ & ND \\
\hline Trench Wall, Center & $121.92(48)$ & 28 \\
\hline Trench Wall, Center & $152.40(60)$ & ND \\
\hline Trench Wall, West End & $91.44(36)$ & ND \\
\hline Trench Wall, West End & $121.92(48)$ & ND \\
\hline Trench Wall, West End & $152.40(60)$ & ND \\
\hline
\end{tabular}

Source: Eberline, 1977

${ }^{a}$ Picocurie per milliliter 
On September 23, 1976, a transect of sampling holes was dug as shown on Figure 2-17 to determine a vertical profile of concentrations across the spill area. Results of these samples are indicated on the figure.

On October 1, 1976, a final comprehensive sampling of the spill area was made. Three ditches were dug with a backhoe across the area of interest to a depth of $152 \mathrm{~cm}$ (60 in.). The side walls of each ditch were sampled at four locations at depths of 30, 61, 91, 122, and $152 \mathrm{~cm}(12,24,36$, 48 and 60 in.). Figure 2-18 shows the locations and results of these samples. Table 2-6 tabulates the same results. The figure and table indicate that the spill area had been successfully decontaminated.

On October 4, 1976, $0.166 \mathrm{Ci}$ of tritium in waste water and drilling mud were pumped into the Mesaverde formation at a depth of approximately 1,615 to $1,768 \mathrm{~m}(5,300$ to $5,800 \mathrm{ft})$ for disposal. It should be noted that the potable aquifers above this depth were previously cemented off during emplacement drilling.

\section{Decontamination Work Area}

The decontamination work area included the area around and under the decontamination pan as well as the adjacent area used to convert low-level, tritiated water into steam for disposal. After work in the area was completed, the soil was sampled at 25 points on the surface and at a $30-\mathrm{cm}$ (12-in.) depth, giving a total of 50 samples. Results of sample analyses and the locations are shown on Figure 2-19. The results are also tabulated on Table 2-7. Note that all concentrations of tritium in soil moisture were negligible except for two locations where the highest of four samples was $10,953 \mathrm{pCi} / \mathrm{mL}$, still well below the guideline. This anomaly is explained by the fact that a small hole was punched through the pan at that location. A small amount of the decontaminated liquid leaked to the soil before the hole could be repaired.

\section{R-E Wellhead Area}

No contamination had ever been detected in the recirculating fluid during the destemming operation, nor were the wellhead or workover rig contaminated, therefore, there was little or no potential for soil contamination around the wellhead. However, since this area had not been previously sampled, soil samples were taken from the surface and from a 30-cm (12-in.) depth at the four corners, $0.3048 \mathrm{~m}(1 \mathrm{ft})$ from the cement cellar, giving a total of eight samples. Locations and analytical results are shown on Figure 2-20, and the results are tabulated in Table 2-8. Concentrations of tritium in soil moisture were negligible, as expected. 


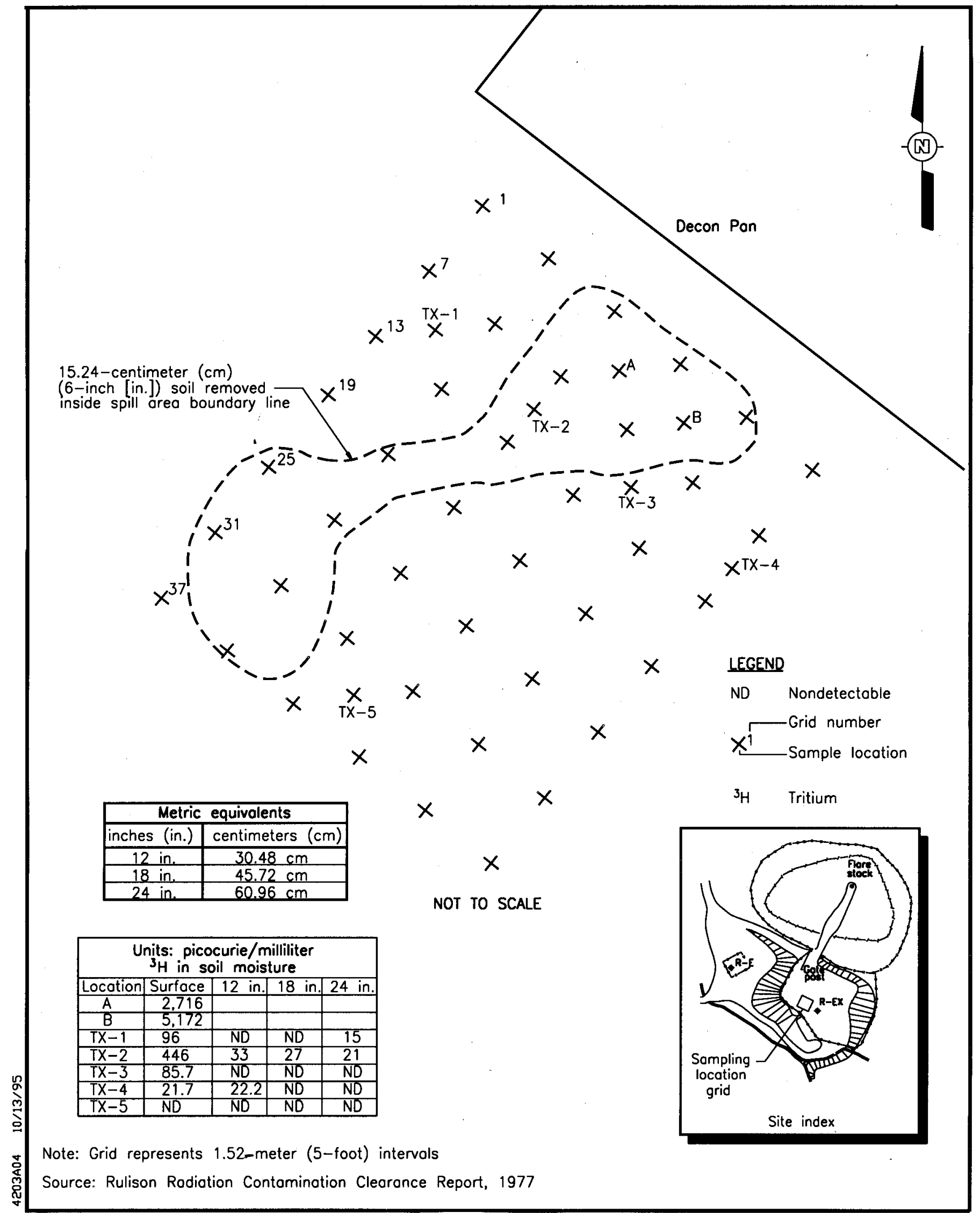

15.24-centimeter $(\mathrm{cm})$

6-inch [in]) soil removed

inside spil 


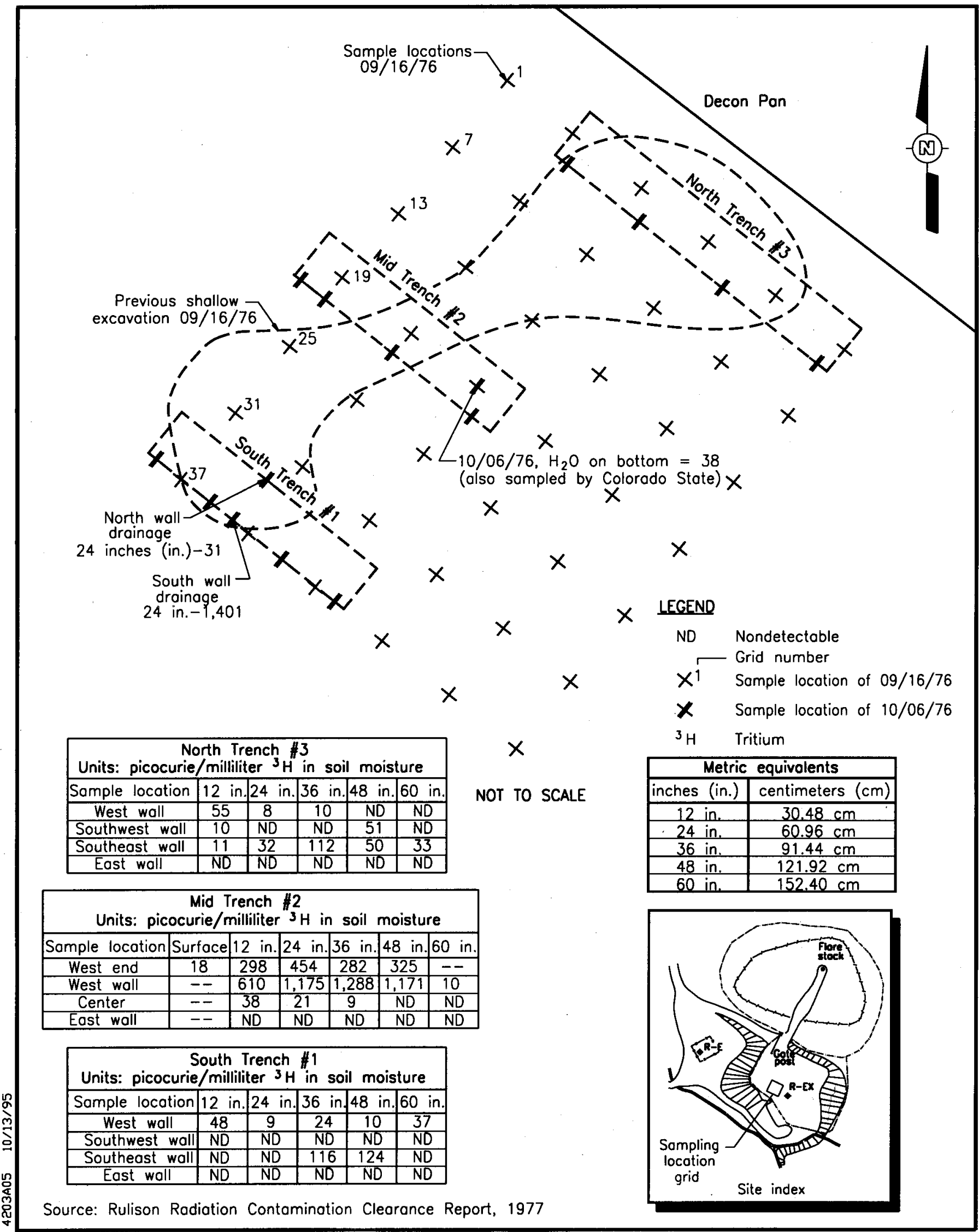

Source: Rulison Radiation Contamination Cleorance Report, 1977

Figure 2-18

Rulison Separator Spill (Trenches), October 1, 1976, Garfield County, Colorado 


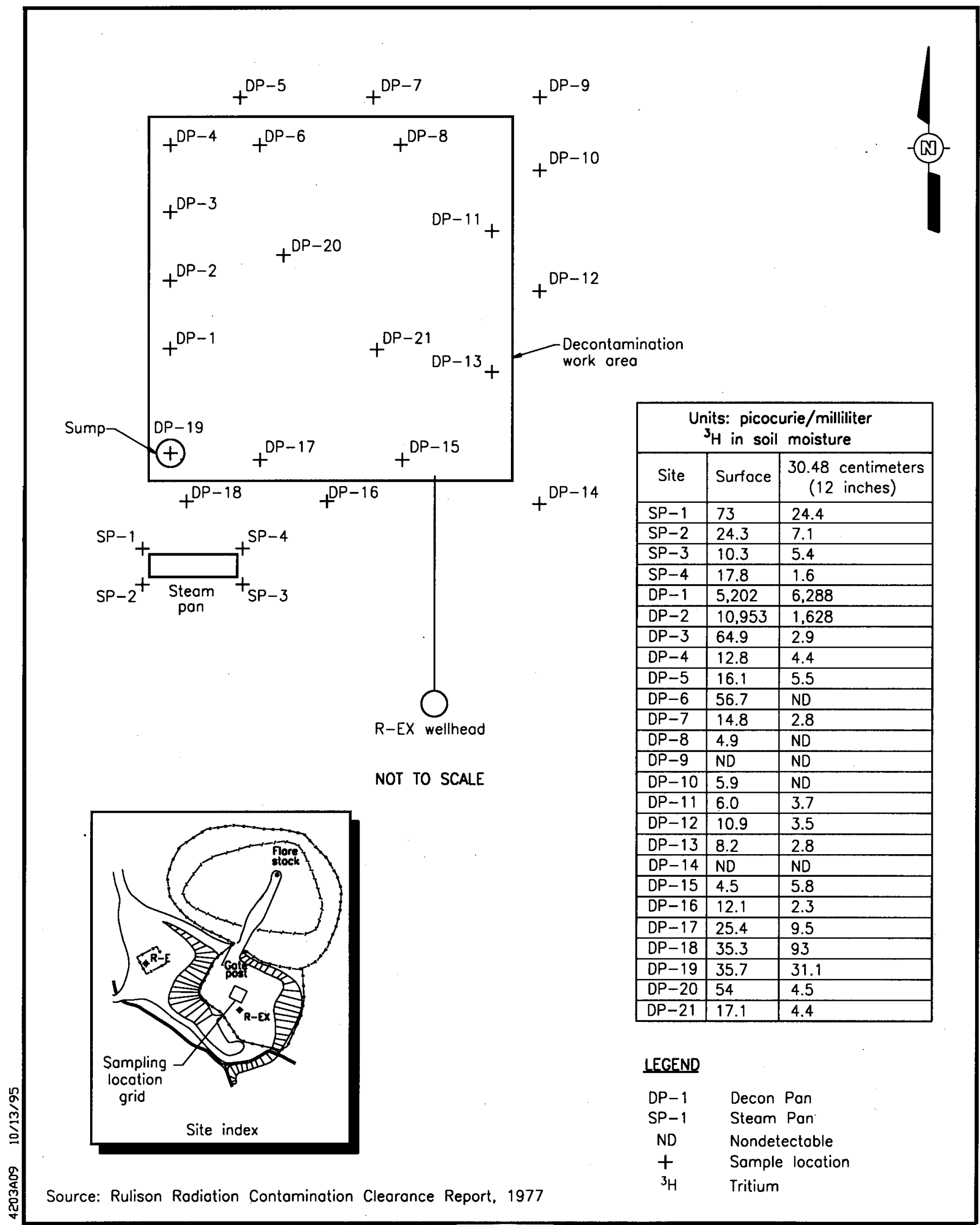

Figure 2-19

Soil Sampling, Rulison R-EX Decon Pan Area,

October 7, 1976, Garfield County, Colorado 


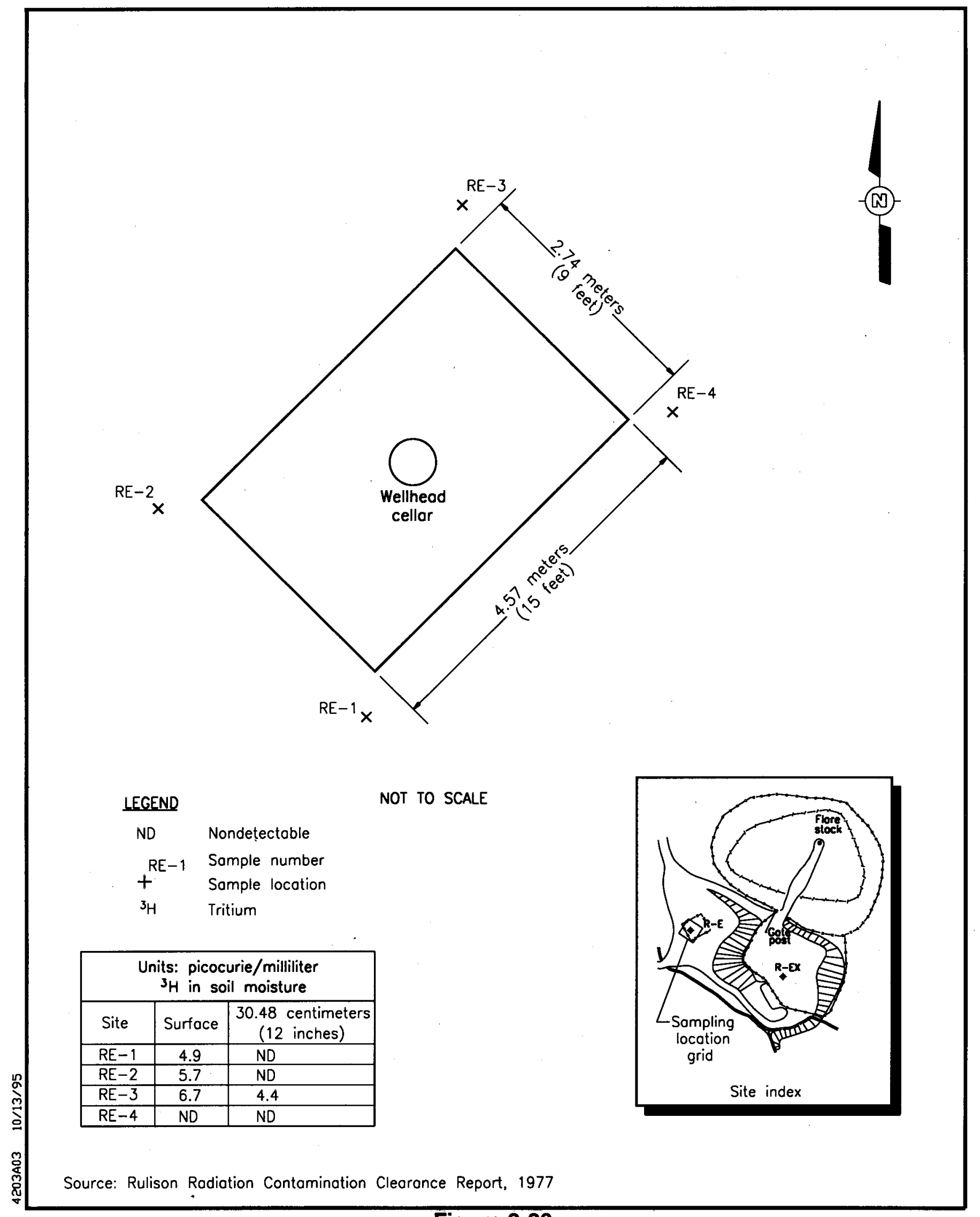

Figure 2-20

Soil Sampling, Rulison R-E Wellhead Cellar Area, October 7, 1976, Garfield County, Colorado 
Table 2-6

Tritium in Soil at Spill Area after Decontamination - October 1976

(Page 1 of 2)

\begin{tabular}{|c|c|c|}
\hline $\begin{array}{c}\text { Sample } \\
\text { Identification }\end{array}$ & $\begin{array}{c}\text { Sampling Depth } \\
\text { centimeters (inches) }\end{array}$ & $\begin{array}{c}\mathrm{pCi} / \mathrm{ml}^{\mathrm{a}} \text { in } \\
\text { Soil Moisture }\end{array}$ \\
\hline South Trench \#1, East Wall & $30.48(12)$ & $\mathrm{ND}$ \\
\hline South Trench \#1, East Wall & $60.96(24)$ & ND \\
\hline South Trench \#1, East Wall & $91.44(36)$ & ND \\
\hline South Trench \#1, East Wall & $121.92(48)$ & ND \\
\hline South Trench \#1, East Wall & $152.40(60)$ & ND \\
\hline South Trench \#1, S.E. Wall & $30.48(12)$ & ND \\
\hline South Trench \#1, S.E. Wall & $60.96(24)$ & ND \\
\hline South Trench \#1, S.E. Wall & $91.44(36)$ & 116 \\
\hline South Trench \#1, S.E. Wall & $121.92(48)$ & 124 \\
\hline South Trench \#1, S.E. Wall & $152.40(60)$ & $\mathrm{ND}$ \\
\hline South Trench \#1, S. Drain Area & $60.96(24)$ & 1,401 \\
\hline South Trench \#1, S.W. Wall & $30.48(12)$ & $\mathrm{ND}$ \\
\hline South Trench \#1, S.W. Wall & $60.96(24)$ & $\mathrm{ND}$ \\
\hline South Trench \#1, S.W. Wall & $91.44(36)$ & $\mathrm{ND}$ \\
\hline South Trench \#1, S.W. Wall & $121.92(48)$ & $\mathrm{ND}$ \\
\hline South Trench \#1, S.W. Wall & $152.40(60)$ & $\mathrm{ND}$ \\
\hline South Trench \#1, West Wall & $30.48(12)$ & 48 \\
\hline South Trench \#1, West Wall & $60.96(24)$ & 9 \\
\hline South Trench \#1, West Wall & $91.44(36)$ & 24 \\
\hline South Trench \#1, West Wall & $121.92(48)$ & 10 \\
\hline South Trench \#1, West Wall & $152.40(60)$ & 37 \\
\hline Mid Trench \#2, East Wall & $30.48(12)$ & ND \\
\hline Mid Trench \#2, East Wall & $60.96(24)$ & ND \\
\hline Mid Trench \#2, East Wall & $91.44(36)$ & ND \\
\hline Mid Trench \#2, East Wall & $121.92(48)$ & ND \\
\hline Mid Trench \#2, East Wall & $152.40(60)$ & ND \\
\hline Mid Trench \#2, Center & $30.48(12)$ & 38 \\
\hline Mid Trench \#2, Center & $60.96(24)$ & 21 \\
\hline Mid Trench \#2, Center & $91.44(36)$ & 9 \\
\hline Mid Trench \#2, Center & $121.92(48)$ & $N D$ \\
\hline Mid Trench \#2, Center & $152.40(60)$ & ND \\
\hline Mid Trench \#2, West Wall & $30.48(12)$ & 610 \\
\hline Mid Trench \#2, West Wall & $60.96(24)$ & 1,175 \\
\hline Mid Trench \#2, West Wall & $91.44(36)$ & 1,288 \\
\hline Mid Trench \#2, West Wall & $121.92(48)$ & 1,171 \\
\hline Mid Trench \#2, West Wall & $152.40(60)$ & 10 \\
\hline Mid Trench \#2, West End & Surface & 18 \\
\hline Mid Trench \#2, West End & $30.48(12)$ & 298 \\
\hline Mid Trench \#2, West End & $60.96(24)$ & 454 \\
\hline Mid Trench \#2, West End & $91.44(36)$ & 282 \\
\hline Mid Trench \#2, West End & $121.92(48)$ & 352 \\
\hline
\end{tabular}

Refer to footnotes at end of table 
Table 2-6

Tritium in Soil at Spill Area after Decontamination - October 1976

(Page 2 of 2)

\begin{tabular}{|c|c|c|}
\hline $\begin{array}{c}\text { Sample } \\
\text { Identification }\end{array}$ & $\begin{array}{l}\text { Sampling Depth } \\
\text { centimeters (inches) }\end{array}$ & $\begin{array}{c}\mathrm{pCi} / \mathrm{ml}^{\mathbf{2}} \text { in } \\
\text { Soil Moisture }\end{array}$ \\
\hline North Trench \#3, East Wall & $30.48(12)$ & $\begin{array}{l}\mathrm{ND} \\
\end{array}$ \\
\hline North Trench \#3, East Wall & $60.96(24)$ & ND \\
\hline North Trench \#3, East Wall & $91.44(36)$ & ND \\
\hline North Trench \#3, East Wall & $121.92(48)$ & ND \\
\hline North Trench \#3, East Wall & $152.40(60)$ & ND \\
\hline North Trench \#3, S.E. Wall & $30.48(12)$ & 11 \\
\hline North Trench \#3, S.E. Wall & $60.96(24)$ & 23 \\
\hline North Trench \#3, S.E. Wall & $91.44(36)$ & 112 \\
\hline North Trench \#3, S.E. Wall & $121.92(48)$ & 50 \\
\hline North Trench \#3, S.E. Wall & $152.40(60)$ & 33 \\
\hline North Trench \#3, S.W. Wall & $30.48(12)$ & 10 \\
\hline North Trench \#3, S.W. Wall & $60.96(24)$ & ND \\
\hline North Trench \#3, S.W. Wall & $91.44(36)$ & ND \\
\hline North Trench \#3, S.W. Wall & $121.92(48)$ & 51 \\
\hline North Trench \#3, S.W. Wall & $152.40(60)$ & ND \\
\hline North Trench \#3, West Wall & $30.48(12)$ & 55 \\
\hline North Trench \#3, West Wall & $60.96(24)$ & 8 \\
\hline North Trench \#3, West Wall & $91.44(36)$ & 10 \\
\hline North Trench \#3, West Wall & $121.92(48)$ & ND \\
\hline North Trench \#3, West Wall & $152.40(60)$ & ND \\
\hline
\end{tabular}

Source: Eberline, 1977

a Picocurie per millititer 
Table 2-7

Tritium in Decontamination Work Area Soil

(Page 1 of 2)

\begin{tabular}{|c|c|c|}
\hline $\begin{array}{c}\text { Sample } \\
\text { Identification }\end{array}$ & $\begin{array}{c}\text { Sampling Depth } \\
\text { centimeters (inches) }\end{array}$ & $\begin{array}{c}\mathrm{pCi} / \mathrm{ml}^{\mathrm{a}} \text { in } \\
\text { Soil Moisture }\end{array}$ \\
\hline DP-1 & Surface & 5,202 \\
\hline DP-1 & $30.48(12)$ & 6,288 \\
\hline DP-2 & Surface & 10,953 \\
\hline DP-2 & $30.48(12)$ & 1,628 \\
\hline DP-3 & Surface & 64.9 \\
\hline DP-3 & $30.48(12)$ & 2.9 \\
\hline DP-4 & Surface & 12.8 \\
\hline DP-4 & $30.48(12)$ & 4.4 \\
\hline DP-5 & Surface & 16.1 \\
\hline DP-5 & $30.48(12)$ & 5.5 \\
\hline DP-6 & Surface & 56.7 \\
\hline DP-6 & $30.48(12)$ & $\mathrm{ND}$ \\
\hline DP-7 & Surface & 14.8 \\
\hline DP-7 & $30.48(12)$ & 2.8 \\
\hline DP-8 & Surface & 4.9 \\
\hline DP-8 & $30.48(12)$ & $\mathrm{ND}$ \\
\hline DP-9 & Surface & $\mathrm{ND}$ \\
\hline DP-9 & $30.48(12)$ & $N D$ \\
\hline DP-10 & Surface & 5.9 \\
\hline DP-10 & $30.48(12)$ & $\mathrm{ND}$ \\
\hline DP-11 & Surface & 6 \\
\hline DP-11 & $30.48(12)$ & 3.7 \\
\hline DP-12 & Surface & 10.9 \\
\hline DP-12 & $30.48(12)$ & 3.5 \\
\hline DP-13 & Surface & 8.2 \\
\hline DP-13 & $30.48(12)$ & 2.8 \\
\hline DP-14 & Surface & $\mathrm{ND}$ \\
\hline DP-14 & $30.48(12)$ & ND \\
\hline DP-15 & Surface & 4.5 \\
\hline DP-15 & $30.48(12)$ & 5.8 \\
\hline DP-16 & Surface & 12.1 \\
\hline DP-16 & $30.48(12)$ & 2.3 \\
\hline DP-17 & Surface & 25.4 \\
\hline DP-17 & $30.48(12)$ & 9.5 \\
\hline DP-18 & Surface & 35.3 \\
\hline DP-18 & $30.48(12)$ & 93 \\
\hline DP-19 & Surface & 35.7 \\
\hline DP-19 & $30.48(12)$ & 31.1 \\
\hline DP-20 & Surface & 54 \\
\hline DP-20 & $30.48(12)$ & 4.5 \\
\hline DP-21 & Surface & 17.1 \\
\hline
\end{tabular}

Refer to footnotes at end of table 
Table 2-7

Tritium in Decontamination Work Area Soil

(Page 2 of 2)

\begin{tabular}{||c|c|r||}
\hline $\begin{array}{c}\text { Sample } \\
\text { Identification }\end{array}$ & $\begin{array}{c}\text { Sampling Depth } \\
\text { centimeters (inches) }\end{array}$ & $\begin{array}{c}\text { pCi/mi in } \\
\text { Soil Moisture }\end{array}$ \\
\hline \hline DP-21 & $30.48(12)$ & 4.4 \\
\hline SP-1 & Surface & 73 \\
\hline SP-1 & $30.48(12)$ & 24.4 \\
\hline SP-2 & Surface & 24.3 \\
\hline SP-2 & $30.48(12)$ & 7.1 \\
\hline SP-3 & Surface & 10.3 \\
\hline SP-3 & $30.48(12)$ & 5.4 \\
\hline SP-4 & Surface & 17.8 \\
\hline SP-4 & $30.48(12)$ & 1.6 \\
\hline
\end{tabular}

Source: Eberline, 1977

${ }^{\text {a }}$ Picocurie per milliliter 


\section{Table 2-8}

Tritium in Soil at R-E Wellhead - October 1976

\begin{tabular}{|c|c|c||}
\hline $\begin{array}{c}\text { Sample } \\
\text { Identification }\end{array}$ & $\begin{array}{c}\text { Sampling Depth } \\
\text { centimeters (inches) }\end{array}$ & $\begin{array}{c}\mathbf{p C i} / \mathbf{m l}^{\mathbf{a}} \text { in } \\
\text { Soil Moisture }\end{array}$ \\
\hline \hline RE-1 & Surface & 4.9 \\
\hline RE-1 & $30.48(12)$ & ND \\
\hline RE-2 & Surface & 5.7 \\
\hline RE-2 & $30.48(12)$ & ND \\
\hline RE-3 & Surface & 6.7 \\
\hline RE-3 & $30.48(12)$ & 4.4 \\
\hline RE-4 & Surface & ND \\
\hline RE-4 & $30.48(12)$ & ND \\
\hline
\end{tabular}

Source: Eberline, 1977

a Picocurie per milliliter 


\section{Surface Water}

Surface water was sampled at the four locations mentioned: the creek above and below the site, the spring on the site, and the spring down the road from the site. Tritium was not detected at a detection sensitivity of $2 \mathrm{pCi} / \mathrm{mL}$.

\subsection{Aerial Radiological Survey}

An aerial radiological survey was conducted over the Project Rulison Site, 64 km (40 mi) northeast of Grand Junction, Colorado, from July 6 through July 12, 1993. Parallel lines were flown at intervals of $76 \mathrm{~m}(250 \mathrm{ft})$ over a $17-\mathrm{km}^{2}\left(6.5-\mathrm{mi}^{2}\right)$ area at a $61-\mathrm{m}(200-\mathrm{ft})$ altitude surrounding Battlement Creek Valley. The gamma energy spectra obtained were reduced to an exposure rate contour map overlaid on a high altitude aerial photograph of the area. The terrestrial exposure rate varied from 3.5 to 12.5 microroentgens per hour $(\mu \mathrm{R} / \mathrm{hr}$ ) (excluding

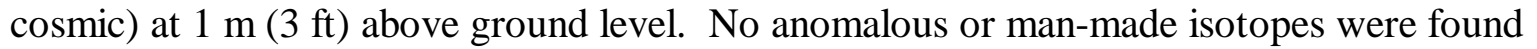
(EG\&G, 1995, p. ii).

\subsubsection{Sampling Summary}

A review of the history of operations at the Rulison Site, the analytical results of sampling programs, and the results of the detailed radiological survey identified the extent of radioactive contamination on the property. The only nuclide of concern was tritium in surface soil moisture. A reasonable and conscientious effort was made to reduce contamination to an amount as low as practicable. Tritium concentrations, where detected, were in most cases negligible and well below the guideline (ERDA, 1976). There is no reason the Rulison Site should change from unrestricted use, subject to applicable subsurface drilling restrictions as stated in Project Rulison Well Plugging and Site Abandonment Plan, NVO-174 (Rev. 1) and Project Rulison Well Plugging and Site Abandonment Final Report, NVO-187 (ERDA, 1976, p. 16; ERDA, 1977, p. 20). 


\subsection{Review of Regulatory Status}

\subsection{Federal Regulatory Overview}

In May 1976, an environmental impact assessment of the Rulison Site was prepared in accordance with the requirements of Title 10 Code of Federal Regulations (CFR), Part 11, dated February 16, 1974, which detailed the procedures to be followed for ERDA implementation of the National Environmental Policy Act of 1969 (ERDA, 1976). The purpose of this assessment was to present a brief description of proposed activities for the Rulison well plugging and site abandonment cleanup and an evaluation of whether an environmental impact statement needed to be prepared.

It was determined from the assessment that the requested action did not constitute a major federal action which significantly affecting the environment, in the sense of the National Environmental Policy Act (NEPA), Section 102(2)(c). At that time, it was determined that no adverse effects to the environment had occurred (ERDA, 1976, p. 18).

In May 1986, Reynolds Electrical \& Engineering Company, Inc., conducted a Hazardous Waste Installation Assessment in which three "operational areas" were sampled, and a report was produced. The descriptive name and actual location of these areas is shown on Figure 3-1. No hazardous materials were detected in any of the samples collected at the Rulison Site (Fauver, 1986, p. 30). The objective of the Hazardous Waste Installation Assessment Project was to identify and evaluate inactive sites at DOE/NV installations where hazardous substances may have been released into the environment. These "Installation Assessments" were the first phase of the DOE/NV effort to satisfy DOE Order 5480/14, which required that federal facilities comply with the CERCLA.

A CERCLA Preliminary Assessment was prepared by the DRI for the Rulison Site in 1988. The CERCLA/Superfund Amendments and Reauthorization Act provides that all EPA regulations and criteria pertaining to inactive hazardous waste sites are applicable to U.S. Government facilities. Included among the provisions of these acts are requirements for a preliminary assessment of each facility and an evaluation based on the same Hazard Ranking System (HRS) that is applied to nonfederal facilities. 


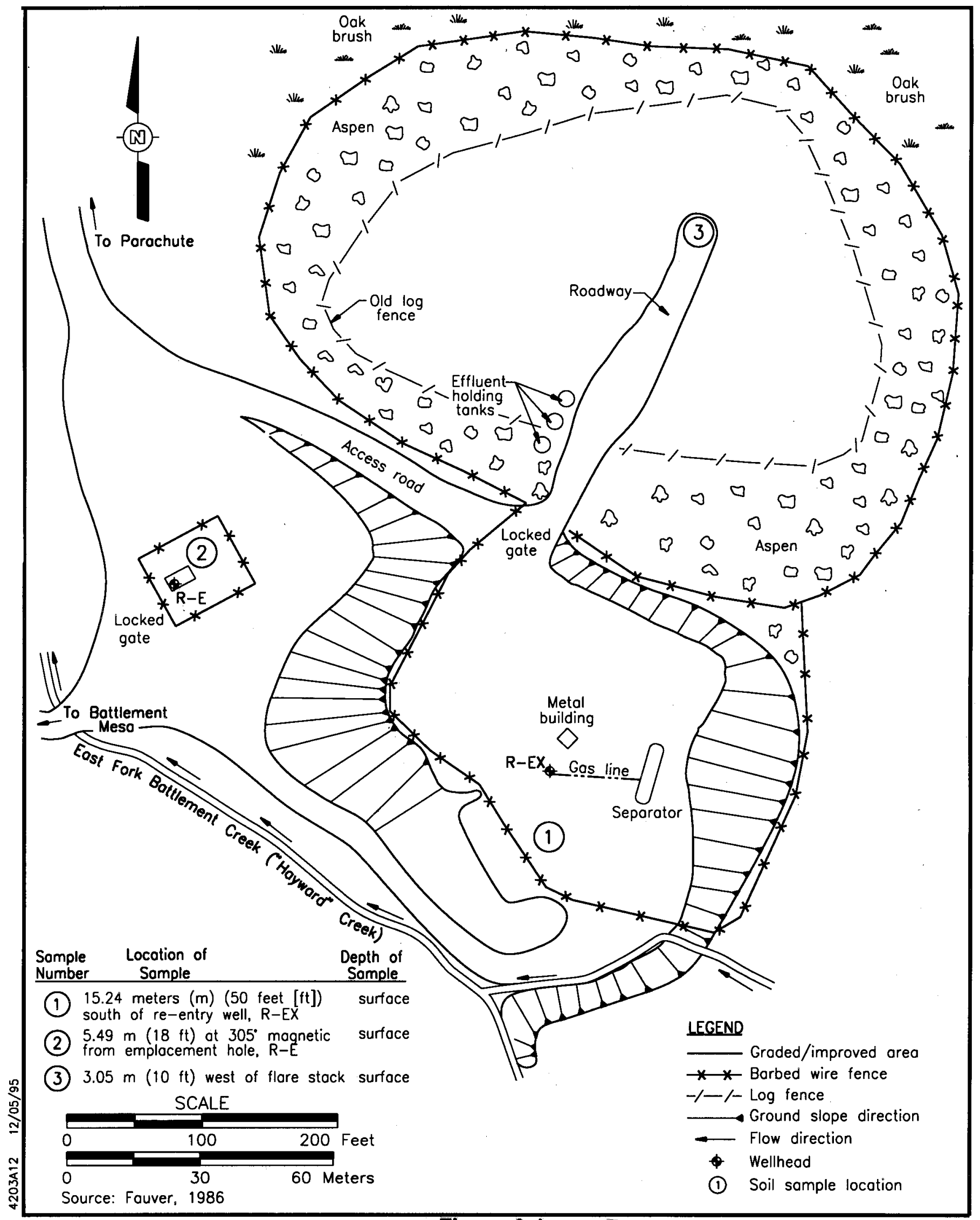

Figure 3-1

Soil Sampling, Potential Hazardous Waste Release Sites at the Rulison Test Site, September 1976, Garfield County, Colorado 
The 1988 preliminary assessment concluded that radiation was released to the environment during Project Rulison production testing. The R-E and R-EX wells were plugged to prevent the escape of radiation, and the explosive device was detonated 2,568 $\mathrm{m}(8,426 \mathrm{ft})$ below ground surface in the Mesa Verde formation. Given the extremely low permeability of this formation, radionuclide migration should be very limited; however, surface and subsurface water quality monitoring is still being conducted near the Rulison Site. A preliminary HRS score for Project Rulison was calculated to be 15.12 , well below the score of 28.5 which is required for a site to be placed on the National Priorities List. The only contributing score was from the air route due to the release of radioactivity during gas production testing. An extensive on- and off-site radiation surveillance effort failed to detect any radioactivity other than tritium and krypton in the environment. Typically, the concentrations of these isotopes in the air were around one 10-millionth of their concentration in the gas (DOE, 1984). Because the emplacement and re-entry holes have been plugged, it is unlikely that further air releases will occur.

DOE Order 5440.1E, implementing NEPA, requires that the presence of environmentally sensitive resources such as cultural resources, sensitive species, wetlands, and floodplains be determined so that the appropriate level of NEPA documentation can be established and adequate mitigation measures implemented. IT Corporation (IT) prepared several reports documenting the surveys conducted for these environmentally sensitive resources (IT, 1993a; IT, 1993b; IT, 1993c).

\subsection{State Regulatory Overview}

\subsubsection{Property of Historic, Archaeological, or Architectural Significance}

The Colorado Office of Archaeology and Historic Preservation instituted a file search on December 22, 1992, and IT conducted a Class II Cultural Resources Field Survey on July 1, 1993 (IT, 1993a). The purpose of the investigation was to comply with federal mandates pertaining to the historic preservation of cultural resources, including Section 106 of the National Historic Preservation Act, as implemented by the Advisory Council on Historic Preservation, (Title 36 CFR Part 800; the Archaeological and Historic Preservation Act), Executive Order 11593, and the American Indian Religious Freedom Act. The DOE regulations contained in Title 10 CFR Part 1021 also require compliance with historic preservation mandates. The Project Rulison survey was conducted on private lands under the auspices of State of Colorado Archaeological Permit No. 93-48, the survey was conducted to evaluate the potential impacts that could occur as a result of performing site characterization or possible remedial activities at the Rulison Site. 
The survey resulted in one historic, isolated find consisting of a cast iron stove and one historic monument, the Rulison Site SGZ. The Rulison Site SGZ monument (5GF1656) should be considered eligible for nomination to the National Register of Historic Places along with three other similar sites in Colorado and New Mexico. The monument inscription at SGZ reads:

No excavation, drilling, and/or removal of subsurface materials to a depth of 12,450 ft is permitted within Lot 11, NE 1/4 SW 1/4 of Section 25, Township 7 South, Range 95 West, 6th Principal Meridian, Garfield County, Colorado, without U.S. Government permission. U.S. Atomic Energy Commission and the Department of the Interior (AEC, 1973a).

Based on the field survey results, it was determined that project field activities could proceed. However, if any cultural material were to be uncovered during any field activities, it is recommended that a qualified archaeologist be called in to assess the find. The U.S. Bureau of Land Management (BLM), the Glenwood Springs Resource Area archaeologist, and the Office of Archaeology and Historic Preservation should also be notified under those circumstances.

\subsubsection{Special Sources of Water}

No water sources within this area are vital to the region. The East Fork of Battlement Creek is used, in part, to irrigate land downstream from the Rulison Site (USGS, 1970, p. 7).

Groundwater resources around the Rulison Site occur in surficial deposits such as fan gravel and terraces. These deposits are reportedly "the only sources of usable groundwater near the Rulison Site" (USGS, 1970. p. 9). Available records do not indicate the existence of a sole-source aquifer or a well-head protection area at this site. Refer to Figure 2-1 which shows the wells in the vicinity of the Rulison Site. 


\subsection{Surrounding Land Use}

The Rulison Site is located a few miles outside of the White River National Forest and approximately nine miles north of the Grand Mesa National Forest. No areas within the Rulison Site are federal or state property (BLM, 1980 and 1986; USGS, 1987). Surface ground zero is located on the approximately 16-hectare (40-acre) lot owned by Mr. Cary Weldon; however, the U.S. Government retains control of the subsurface rights. The former drilling effluent pond is on land jointly owned by Ms. Cristy Koeneke and Mr. Craig Hayward. The surrounding land is also privately owned. A map showing current ownership is included as Figure 4-1.

The surrounding land is currently used for recreational purposes (e.g., hunting and fishing) and cattle grazing. During the summer months, a residence located approximately $427 \mathrm{~m}(1,400 \mathrm{ft})$ from the former drilling effluent pond is occupied. Future use of this land is likely to also include recreational and grazing applications.

The closest population center to the Rulison Site is the town of Parachute, which is located approximately $12 \mathrm{~km}(8 \mathrm{mi})$ north of the site and has a recorded population of 660 (Rand McNally, 1993). 


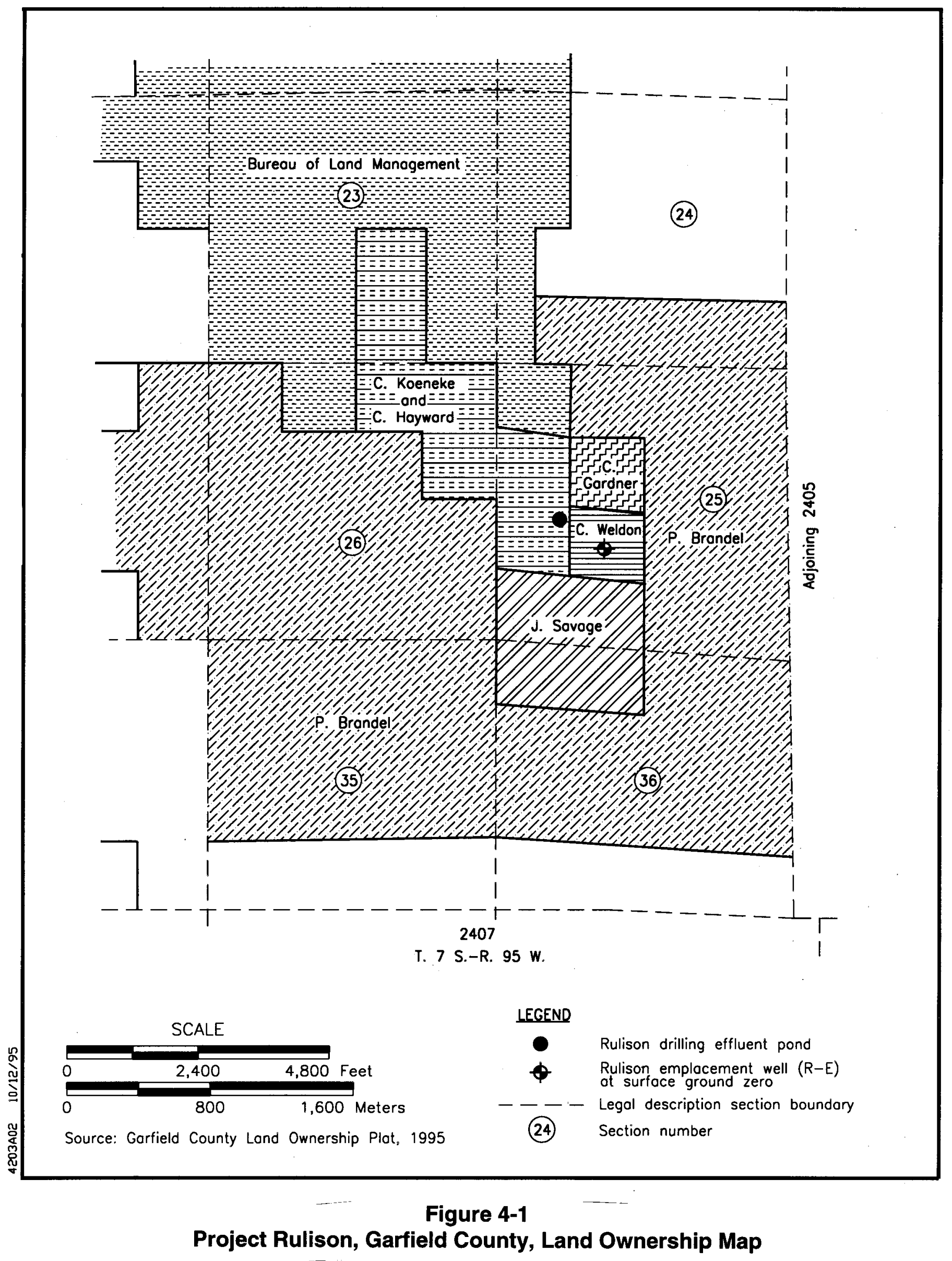

4-2 


\subsection{Physical Environment}

\subsection{Meteorology}

West-central Colorado is generally classified as semiarid, with low precipitation and relative humidity, warm summer temperatures, and abundant sunshine (Marlatt, 1973). Winds are generally from the west, but fail to carry much moisture from the Pacific Ocean past mountain barriers. The average annual precipitation for the Rulison Site is $50 \mathrm{~cm}(20 \mathrm{in}$.) and the temperature ranges from -10 degrees Fahrenheit $\left({ }^{\circ} \mathrm{F}\right)$ to $+98^{\circ} \mathrm{F}\left(-23\right.$ degrees Celsius $\left[{ }^{\circ} \mathrm{C}\right]$ to $\left.+37^{\circ} \mathrm{C}\right)$. Annual precipitation ranges from $25 \mathrm{~cm}(10 \mathrm{in}$.) at elevations of 1,524 $\mathrm{m}(5,000 \mathrm{ft})$ above mean sea level (amsl) to $64 \mathrm{~cm}$ (25 in.) at 2,439 $\mathrm{m}(8,000 \mathrm{ft})$ amsl. Winter snowfall may exceed $256 \mathrm{~cm}$ (100 in.) on plateau tops (Marlatt, 1973). The length of the growing season at Parachute is 150 days (Brooks et al., 1933). Movement of air away from the Rulison Site is controlled by the valley drainage winds and daily up-slope winds in both the Battlement Creek Valley and the Colorado River Valley. The regional gradient wind generally blows east-northeast, above the topographical features (DOE, 1984, p. 3).

The evaporative demand on the north slope of Battlement Mesa is fairly low compared to that of the area north of the Colorado River (Marlatt, 1973). Moisture has a chance to soak into the volcanic soils; thus, the vegetative community is well developed. This enables the community to support a variety of faunal species.

\subsection{Biota}

\subsubsection{Sensitive Species Survey}

The Rulison Site has the potential for supporting a large number of wildlife species. Uplands, wetlands, and surface water bodies offer numerous resources for the organisms that use the site. Food resources for deer, rodents, birds, and canids are abundant. Acorns from the Gambel oak and seeds from the conifers provide mast for herbivores which, in turn, are prey for the carnivores. The beavers on the site feed primarily on aspen. Cover required for all wildlife species is abundant and varied.

A Level I reconnaissance survey for sensitive species was conducted at the Rulison Site in June 1993 (IT, 1993b). For this survey, sensitive species included both federal- and state-listed 
threatened and endangered species and candidate species. Tables 5-1 through 5-3 list the various species found on the Rulison Site during this survey.

In addition, suitable habitat and food resources for several endangered and candidate bird species were identified; however, none of these species were observed during the site reconnaissance. The tiger salamander (Ambystoma tigrinium), which is a State listed species, was observed in the drilling effluent pond. However, communication with the Colorado Division of Wildlife indicated that the tiger salamander is not a species of special concern in that area (Nessler, 1995).

\subsubsection{Vegetation}

The habitats present at the Rulison Site are a combination of Rocky Mountain Montane and Subalpine forest (Whitney, 1992). At lower elevations (2,290 to 2,440 m [7,500 to 8,000 ft]), the dominant montane vegetation consists of quaking aspen (Populus tremuloides), Colorado blue spruce (Pecea pungens), willow (Salix spp.), lodgepole pine (Pinus contorta), Gambel oak (Quercus gambelii), Douglas fir (Pseudotsuga menziesii), mountain mahogany (Cercocarpus montenus), service berry (Amelanchier alnifolia), and mixed mountain shrubs and grasses. The plant species are suitable for grazing of cattle and horses. At elevations greater than 2,440 m $(8,000 \mathrm{ft})$, subalpine species such as sub-alpine fir (Abies lasiocarpa) and Engelmann spruce (Picea engelmannii) become more prevalent in the vegetation.

\subsection{Topography}

The site is located on the north slope of Battlement Mesa, on the upper reaches of Battlement Creek, at an elevation of approximately 2,500 m (8,200 ft) (Figure 5-1). The valley is open to the north-northwest and is bounded on the remaining three sides by steep mountain slopes, which rise to elevations above $2,927 \mathrm{~m}(9,600 \mathrm{ft})$.

\section{Table 5-1}

List of Reptile and Amphibian Species Observed during the Sensitive Species Survey of the Rulison Site, Colorado, June 1993

\begin{tabular}{|c|c|}
\hline Scientific Name & Common Name \\
\hline \multicolumn{2}{|c|}{ Amphibians } \\
\hline $\begin{array}{l}\text { Family: Ambystomatidae } \\
\text { Ambystoma tigrinum }\end{array}$ & Tiger Salamander \\
\hline \multicolumn{2}{|c|}{ Reptiles } \\
\hline $\begin{array}{l}\text { Family: Colubridae } \\
\text { Opheodrys vernacis }\end{array}$ & Smooth Green Snake \\
\hline
\end{tabular}


Table 5-2

\section{List of Bird Species Observed during the Sensitive Species Survey of the Rulison Site, Colorado, June 1993}

\begin{tabular}{|c|c|}
\hline Scientific & Common Name \\
\hline $\begin{array}{l}\text { Family: Accipitridae } \\
\text { Aquila chrysaetos }\end{array}$ & Golden Eagle \\
\hline $\begin{array}{l}\text { Family: Scolopacidae } \\
\text { Calidris minutilla }\end{array}$ & Least Sandpiper \\
\hline $\begin{array}{l}\text { Family: Columbidae } \\
\text { Zenaida macroura }\end{array}$ & Morning Dove \\
\hline $\begin{array}{l}\text { Family: Trochilidae } \\
\text { Selasphorus platycercus }\end{array}$ & Broad-Tailed Hummingbird \\
\hline $\begin{array}{l}\text { Family: Picidae } \\
\text { Sphyrapicus varius } \\
\text { Colaptes auratus }\end{array}$ & $\begin{array}{l}\text { Yellow-Bellied Sapsucker } \\
\text { Northern Flicker }\end{array}$ \\
\hline $\begin{array}{l}\text { Family: Hirundinidae } \\
\text { Tachycineta bicolor }\end{array}$ & Violet-Green Swallow \\
\hline $\begin{array}{l}\text { Family: Corvidae } \\
\text { Corvus corax }\end{array}$ & Common Raven \\
\hline $\begin{array}{l}\text { Family: Paridae } \\
\text { Parus atricapillus }\end{array}$ & Black-Capped Chickadee \\
\hline $\begin{array}{l}\text { Family: Troglodytidae } \\
\text { Troglodytes aedon }\end{array}$ & House Wren \\
\hline $\begin{array}{l}\text { Family: Muscicapidae } \\
\text { Regulus calendula } \\
\text { Catharus guttatus } \\
\text { Turdus migratorius }\end{array}$ & $\begin{array}{c}\text { Ruby-Crowned Kinglet } \\
\text { Hermit Thrush } \\
\text { American Robin }\end{array}$ \\
\hline $\begin{array}{c}\text { Family: Emberizidae } \\
\text { Subfamily: Parulinae } \\
\text { Vermivora virginae } \\
\text { Dendorica petechia } \\
\text { Dendrocia coronata } \\
\text { Oporornis tolmiei }\end{array}$ & $\begin{array}{c}\text { Virginia's Warbler } \\
\text { Yellow Warbler } \\
\text { Yellow-Rumped Warbler [Audubon's form] } \\
\text { MacGillivray's Warbler }\end{array}$ \\
\hline $\begin{array}{l}\text { Subfamily: Emberizinae } \\
\text { Amophila ruficeps } \\
\text { Pooecetes gramineus }\end{array}$ & $\begin{array}{c}\text { Rufous-Crowned Sparrow } \\
\text { Vesper Sparrow }\end{array}$ \\
\hline $\begin{array}{l}\text { Family: Passeridae } \\
\text { Passer domesticus }\end{array}$ & House Sparrow \\
\hline
\end{tabular}




\section{Table 5-3}

List of Mammal Species Observed during the Sensitive

Species Survey of the Rulison Site, Colorado, June 1993

\begin{tabular}{||c|c||}
\hline Scientific Name & Common Name \\
\hline $\begin{array}{c}\text { Family: Leporidae } \\
\text { Sylvilagus nuttalli }\end{array}$ & Mountain Cottontail \\
\hline $\begin{array}{c}\text { Family: Sciuridae } \\
\text { Eutamias minimus } \\
\text { Marmota flaviventris } \\
\text { Citellus lateralis }\end{array}$ & $\begin{array}{c}\text { Least Chipmunk } \\
\text { Yellow-Bellied Marmot } \\
\text { Golden-Mantled Ground Squirrel }\end{array}$ \\
\hline $\begin{array}{c}\text { Family: Castoridae } \\
\text { Castor canadensis }\end{array}$ & Beaver \\
\hline $\begin{array}{c}\text { Family: Procyonidae } \\
\text { Procyon lotor }\end{array}$ & Raccoon \\
\hline $\begin{array}{c}\text { Family: Canidae } \\
\text { Canis familiaris } \\
\text { Canis latrans }\end{array}$ & $\begin{array}{c}\text { Domestic Dog } \\
\text { Coyote }\end{array}$ \\
\hline $\begin{array}{c}\text { Family: Cervidae } \\
\text { Odocoileus hemionus }\end{array}$ & Mule Deer \\
\hline
\end{tabular}

\subsection{Soils}

The Rifle Area, Colorado, Soil Survey (USDA, 1980) indicates two soil types within the 161,880square meters $\left(\mathrm{m}^{2}\right)(40$-acre) site. These include Bucklon-Inchau association loams and Cochetopa loam (Figure 5-2). The character of these soils was confirmed by field analysis of numerous soil borings during the wetlands and floodplain investigation performed in June 1993 (IT, 1993c, p. 4-4). Neither of these soil types constitutes prime agricultural land (Carlson, 1993, personal communication).

Numerous soil borings were taken and field-analyzed during the wetlands delineation. Hydric soils were identified in areas identified as wetlands. These results correspond with the U.S. Department of Agriculture, Soil Conservation Service soils mapping of the Rifle Area.

Bucklon soils make up approximately 55 percent of the map unit and are found on the more steep, convex parts of the landscape. It is a shallow and well-drained soil. Permeability of the Bucklon soil is slow above bedrock. The available water capacity is very low. Effective rooting depth is about 0.25 to $0.51 \mathrm{~m}$ (10 to $20 \mathrm{in}$.). Surface runoff is medium, and the erosion hazard is severe. 


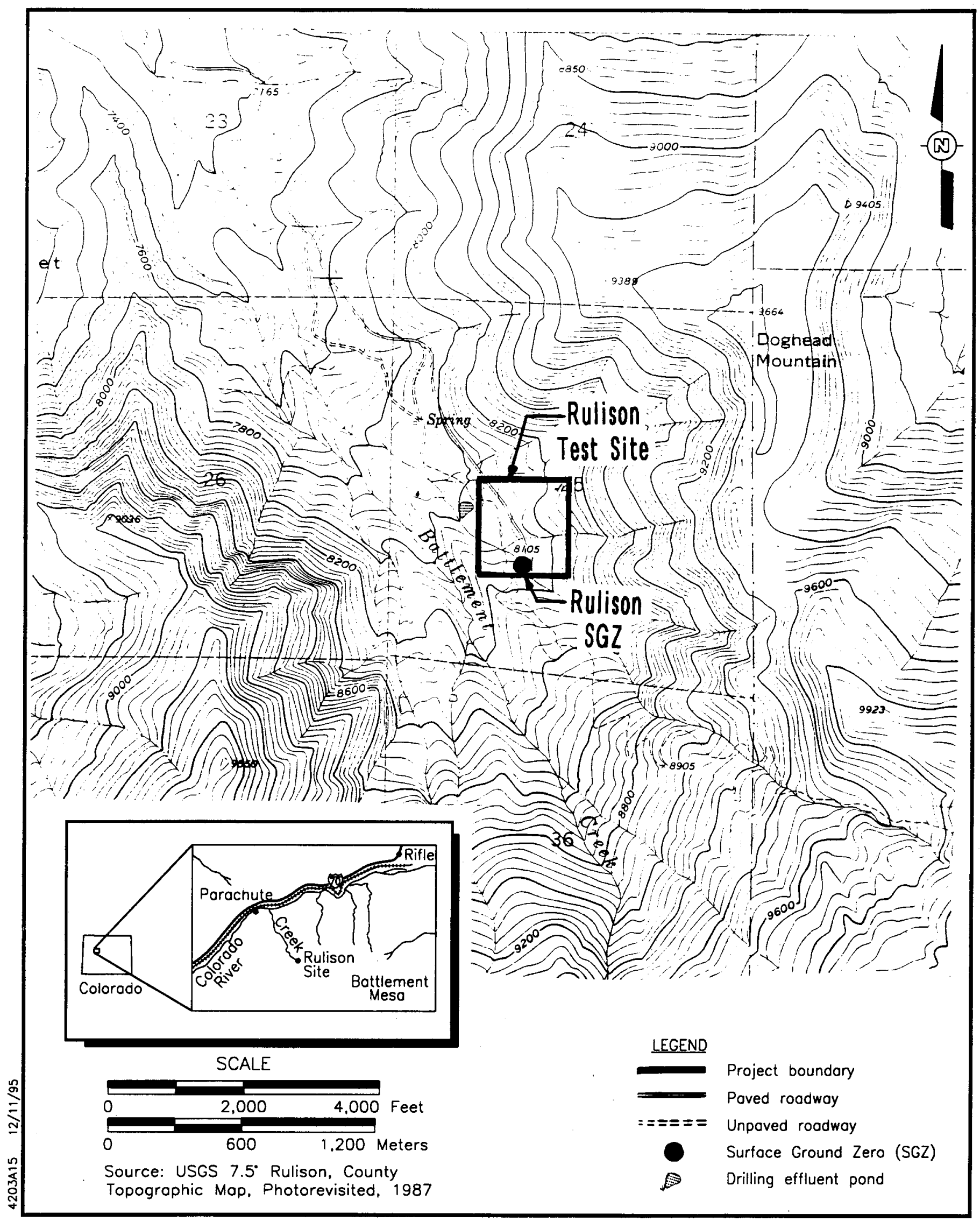

Figure 5-1

Topographic Map of Garfield County, Colorado, Project Rulison 


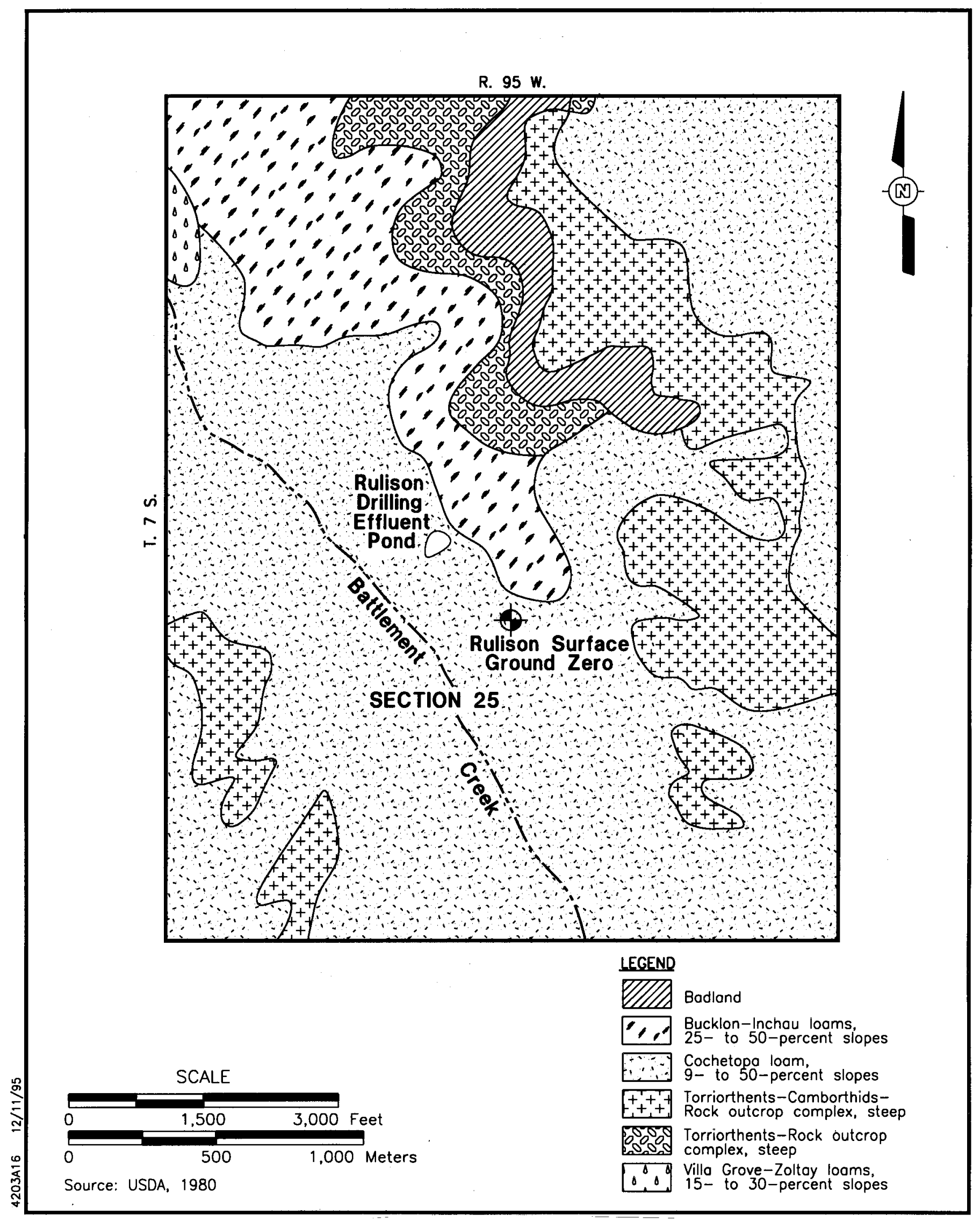

Figure 5-2

U.S. Department of Agriculture Soil Conservation Service Soils Map, Rifle Area, Colorado, Rulison Project 
Inchau soils make up approximately 35 percent of the map unit and occur on the slightly concave parts of the landscape. It is a moderately-deep and well-drained soil. Permeability of Inchau soil is moderate above bedrock, and available water capacity is moderate.

Cochetopa loam is a deep, well-drained soil, and is found on rolling to steep mountainsides and alluvial fans. Elevation ranges from 2,134 to 2,896 $\mathrm{m}$ (7,000 to 9,500 ft). This soil is formed in basaltic alluvium. Permeability is slow, and available water capacity is high. Effective rooting depth is $1.5 \mathrm{~m}$ (60 in.) or more. Surface runoff is slow, and the erosion hazard is severe. High clay content in the soil causes low soil strength and high potential for soil slumping. The subsoil, below a depth of approximately $0.2 \mathrm{~m}$ ( $24 \mathrm{in}$.), consists of stony clay with a low permeability. The Rulison SGZ was constructed in the Cochetopa loam.

\subsection{Geology}

\subsubsection{General Description}

The Rulison Site is located within the Piceance Creek Basin. This northwest-southeast trending, structurally downwarped basin, is delineated primarily by the distribution of the Mesaverde Formation. The basin was structurally deformed by northeast-directed, Laramide-aged, shortening and reactivated, high angle basement structures (CER, 1989; Dickenson and Snyder, 1978). The present basin axis (a synformal fold axis) is oriented approximately northwestsoutheast (Figures 5-3 and 5-4). This present axis is approximately the same as the paleodepositional axis of the Mesaverde Formation. The Rulison Site is located on the southwest limb of the downwarp where the dip of the Mesaverde is about 2 to 3 degrees to the northeast.

\subsubsection{Surficial Geology}

The surficial geology at the Rulison Site consists of Quaternary deposits comprised of talus accumulations, mud flows, fan and pediment gravel, and the alluvium of Battlement Creek and the Colorado River. These deposits range from 6 to $12 \mathrm{~m} \mathrm{(20} \mathrm{to} 40 \mathrm{ft}$ ) in thickness, but locally may be more than 30-m (100-ft) thick. Groundwater occurs in many of these deposits (Voegeli et al., 1970).

Two soil-mapping units have been identified within the $161,880 \mathrm{~m}^{2}$ (40 acres) surrounding the effluent-pond location. These are the Bucklon-Inchau loams and Cochetopa loam described in Section 5.4 (refer to Figure 5-2). The drilling-effluent pond was constructed in the Cochetopa loam. 


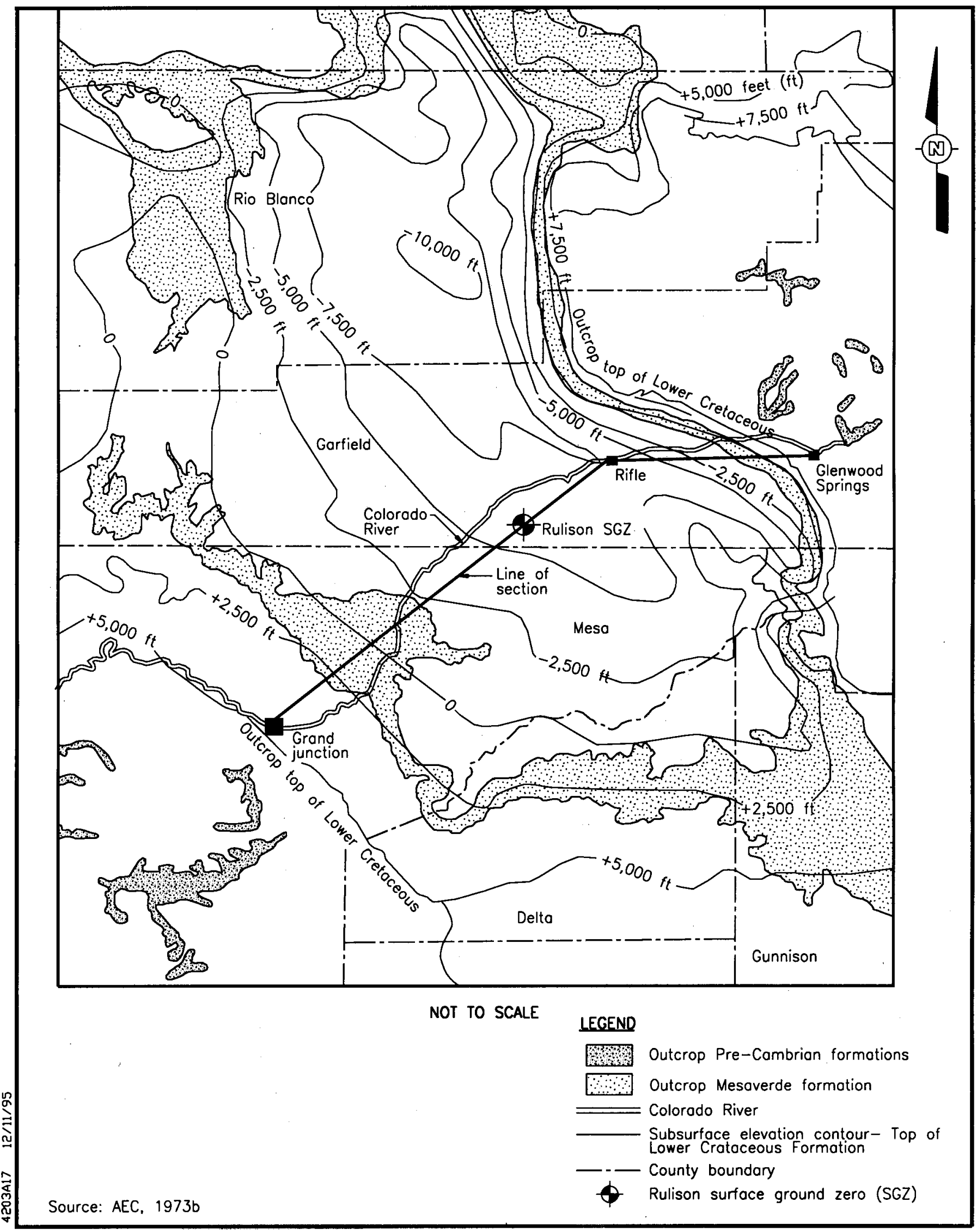

Figure 5-3

Rulison Site, Piceance Creek Basin - Regional Map and Structural Interpretation, Garfield and Mesa Counties, Colorado 


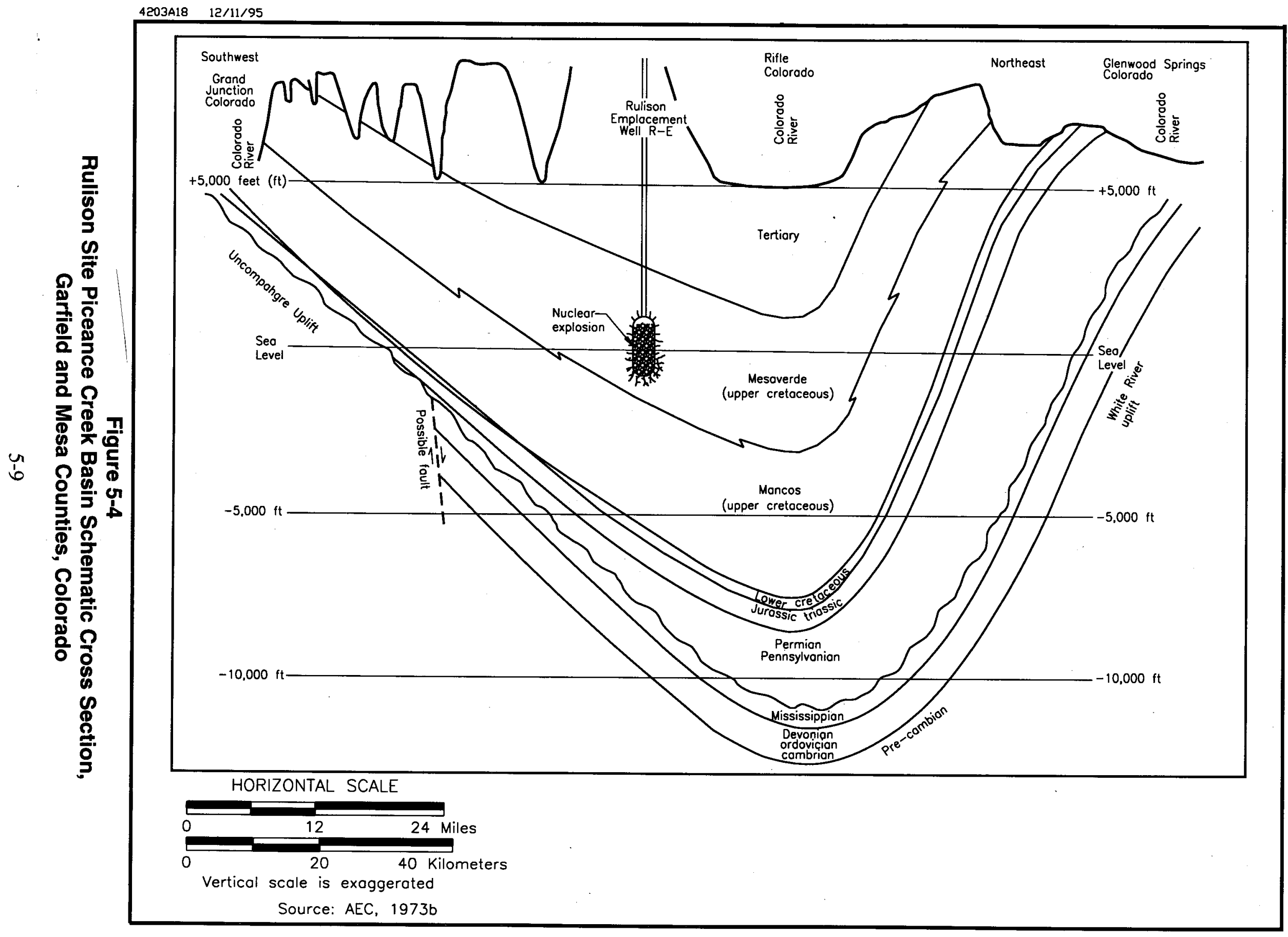




\subsubsection{Subsurface Stratigraphy}

The Piceance Basin contains Precambrian through Holocene stratigraphy. However, because the R-E well only encountered rocks as old as the lower Cretaceous (Mancos Shale), this section will only describe the stratigraphy from the Mancos Shale and above (Figures 5-5 and 5-6).

\subsubsection{Mancos Shale}

The lower Cretaceous Mancos Shale is a marine shale with sparse lenses of sand. Towards the upper half of the Mancos Shale, a transition to a regressive sequence begins that intertongues the shales with the upper Cretaceous Iles Formation within the Mesaverde Group. Overlying the Iles Formation (290 m [900 ft]) the regressive transition continues up into the Williams Fork Formation $(1,067 \mathrm{~m}[3,500 \mathrm{ft}])$ which includes the Tertiary Ohio Creek member $(15 \mathrm{~m}[50 \mathrm{ft}])$ (Lorenz and Rutledge, 1985). The Tertiary units continue with the Fort Union (152 m [500 ft]), Wasatch (1,188 m [3,900 ft]) and Green River Formations (518 m [2,100 ft]). Quaternary basalt flows, locally found in the Rulison area, and alluvial deposits (Pleistocene and recent) unconformably rest on all units.

\subsubsection{Mesaverde Group}

At Rulison, the Mesaverde Group is divided into two Formations: the Iles and Williams Fork (Figure 5-7). The Mesaverde represents a regressive phase from near-shore, deltaic marine (Isles Formation) to non-marine coastal plain, to paludal and meandering river plain, to fluvial environments (Williams Fork) (Lorenz, 1983; Lorenz, 1985; Johnson et al., 1987).

The Isles Formation is characterized by three sand members: the Cocoran and Cozzette intertongued with the Mancos Shale, and the Rollins, a blanket sand that underlies the CameoFairfield Coal of the Williams Fork Formation. The Isles Formation represents a deltaic, shallowmarine sequence (Lorenz, 1983).

Within the Piceance Basin, the thickest sections of the Williams Fork Formation are coincident with the basin axis. The fluvial sand bodies throughout the Williams Fork are laterally extensive and heterogeneous. This suggests that the basin was subsiding during deposition (CER, 1989). In the vicinity of the Rulison Site, the basin axis is oriented east-southeast from which the fluvial paleocurrent directions in the upper Mesaverde can be inferred. Sand-body shapes in the fluvial sequences appear lenticular in cross-section; they are likely longer than the cross-section in the 


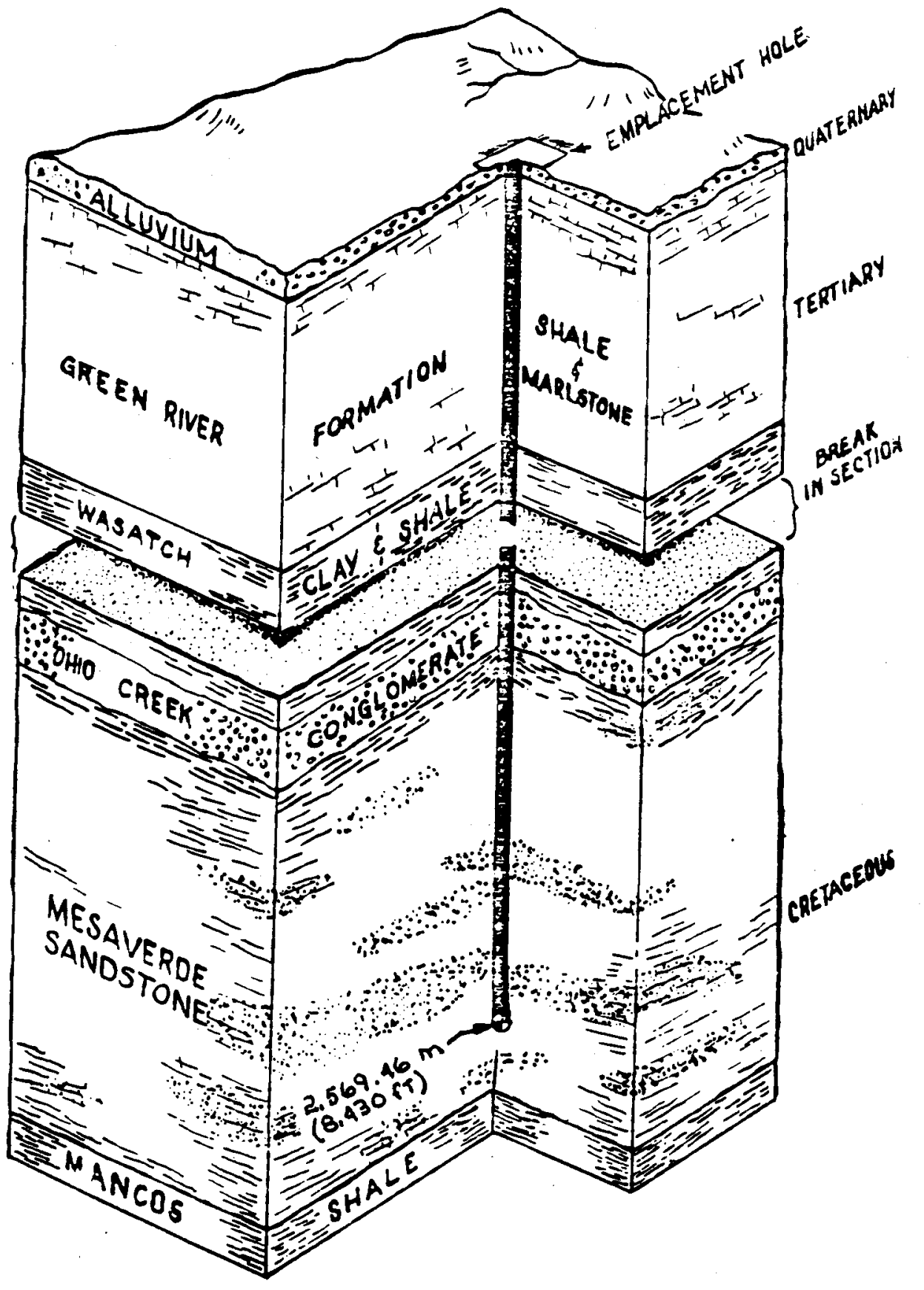

NOT TO SCALE

Source: DRI, 1988

Figure 5-5

Project Rulison Generalized Geologic Cross Section, Garfield County, Colorado 


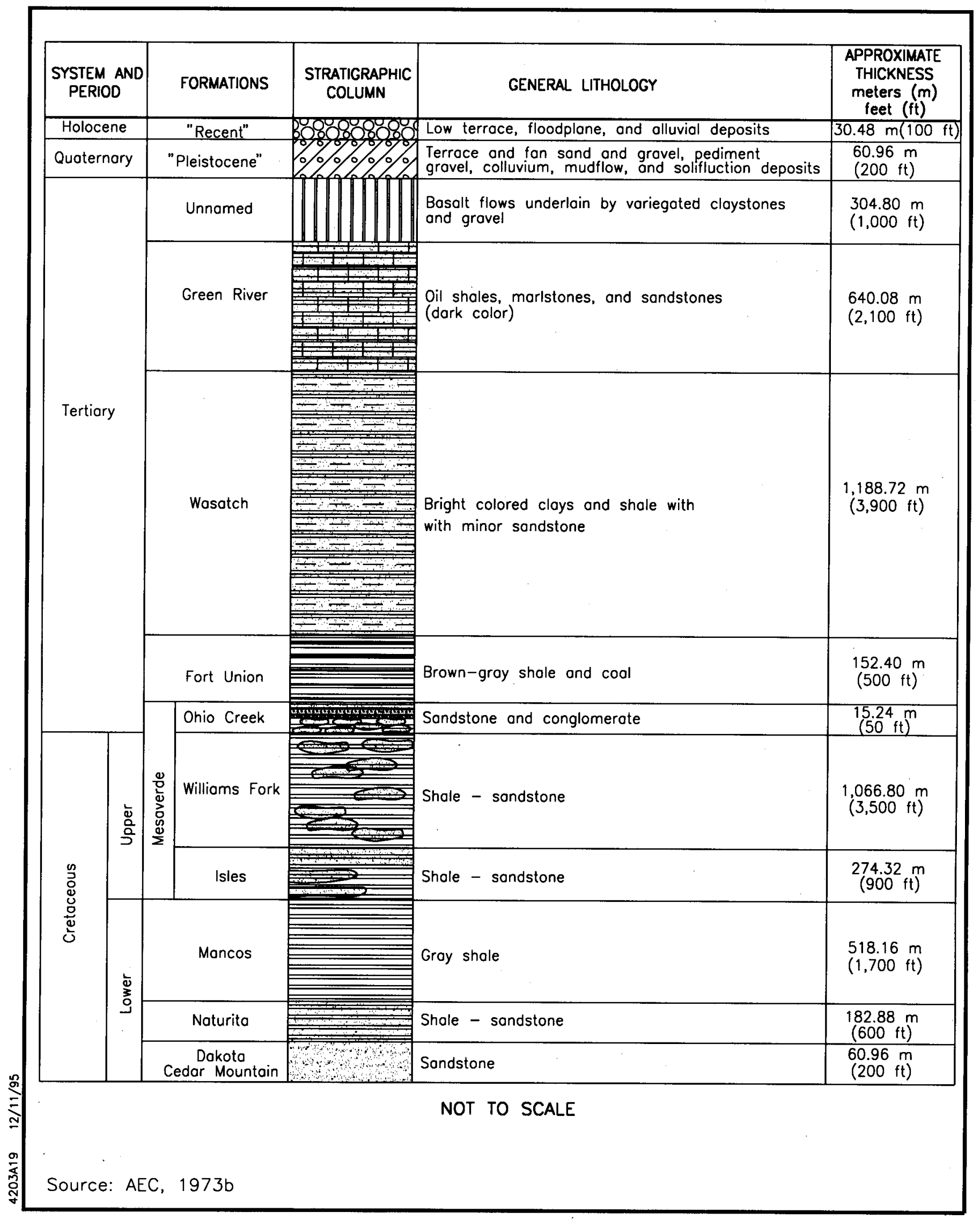

Figure 5-6

Rulison Site Stratigraphic Column, Garfield County, Colorado 


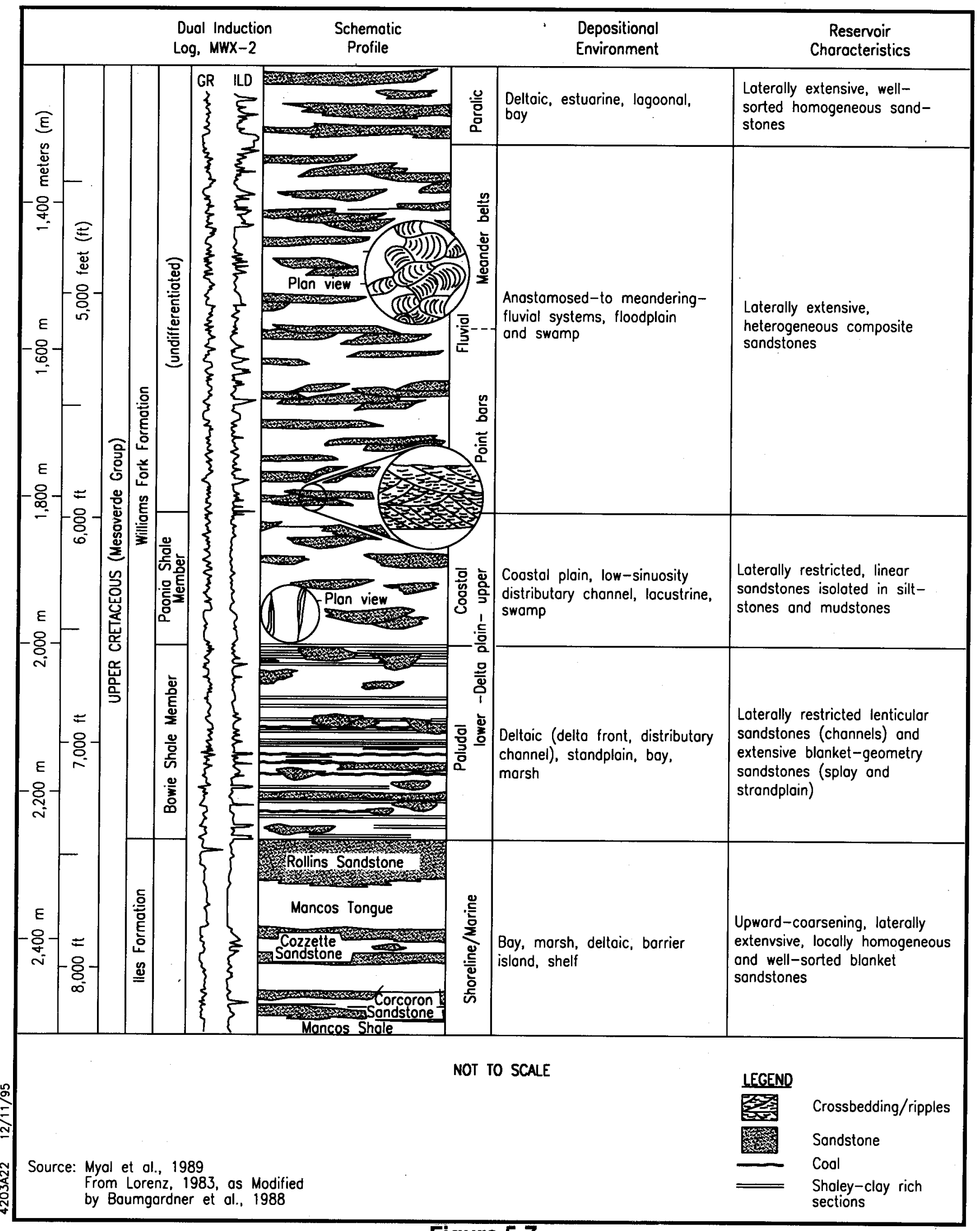

Figure 5-7

Correlation of Paleoenvironmental Depositional Units at the Multi-Well

Experiment Site with Regional Stratigraphic Nomenclature, Garfield County, Colorado 
paleocurrent direction parallel to the basin axis. As the basin continued to subside throughout the Tertiary, the axis of the basin became the deepest zone of the burial (Figure 5-8). This resulted in high compaction and reduced porosity and permeability.

\subsubsection{Tertiary Stratigraphy}

The Tertiary Wasatch and Green River Formations (refer to Figure 5-6) are mostly interbedded shale, marlstone, limestone, and sandstone. Combined, the two formations are over 1,700-m $(5,600-\mathrm{ft})$ thick.

The Wasatch Formation consists of brightly colored clay and shale, but sandstone lenses are common. Locally, minor amounts of conglomerate, pebbly sandstone, limestone, coal, and black cabonaceous shale occur in the formation. The formation is approximately 1,188-m (3,900-ft) thick at the Rulison Site. The Wasatch is not a source of groundwater in the Rulison area.

In and near the Rulison Site, the Green River Formation contains fours members. In ascending order they are: Douglas Creek, Garden Gulch, Parachute Creek, and Evacuation Creek. At the Rulison Site, the Green River Formation is about 518-m (1,700-ft) thick. The most notable unit of the upper Green River, the Parachute Creek member, is an oil shale. This formation is composed of mostly shale and marlstone with minor amounts of sandstone, siltstone, and limestone. Sandy zones in the lower part of the formation may be capable of yielding minor quantities of groundwater at some location in the area (Coffin et al., 1968; Voegeli et al., 1970).

\subsubsection{Natural Gas Production in the Rulison Area}

In the Southern Piceance Basin, natural gas is found in sandstones of both the Wasatch and Mesaverde Formations and in coals of the Mesaverde. The Rulison Site is on the outskirts of the Rulison and Grand Valley gas fields, centered along the Colorado River, which produces gas from both formations.

The closest commercial production wells to the Rulison Site are the Federal 28-95 located $4.3 \mathrm{~km}$ (2.7 mi) west and the Federal 14-95 located $4.34 \mathrm{~km}(2.7 \mathrm{mi})$ to the northwest. The wells are currently operated by Riata Energy, Inc. and Bonneville Fuels Corporation and were drilled in 1961 and 1962, respectively. Both wells produce gas from the Mesaverde Formation. Federal 14-95 had produced a total of 2.12 million $\mathrm{m}^{3}$ (75 million cubic feet [MCF]) by 1988, and the Federal 28-95 39.83 million $\mathrm{m}^{3}$ (375 MCF) by 1993. Both wells produced up to 1993 and are now presently shut in because of the declining gas market. 


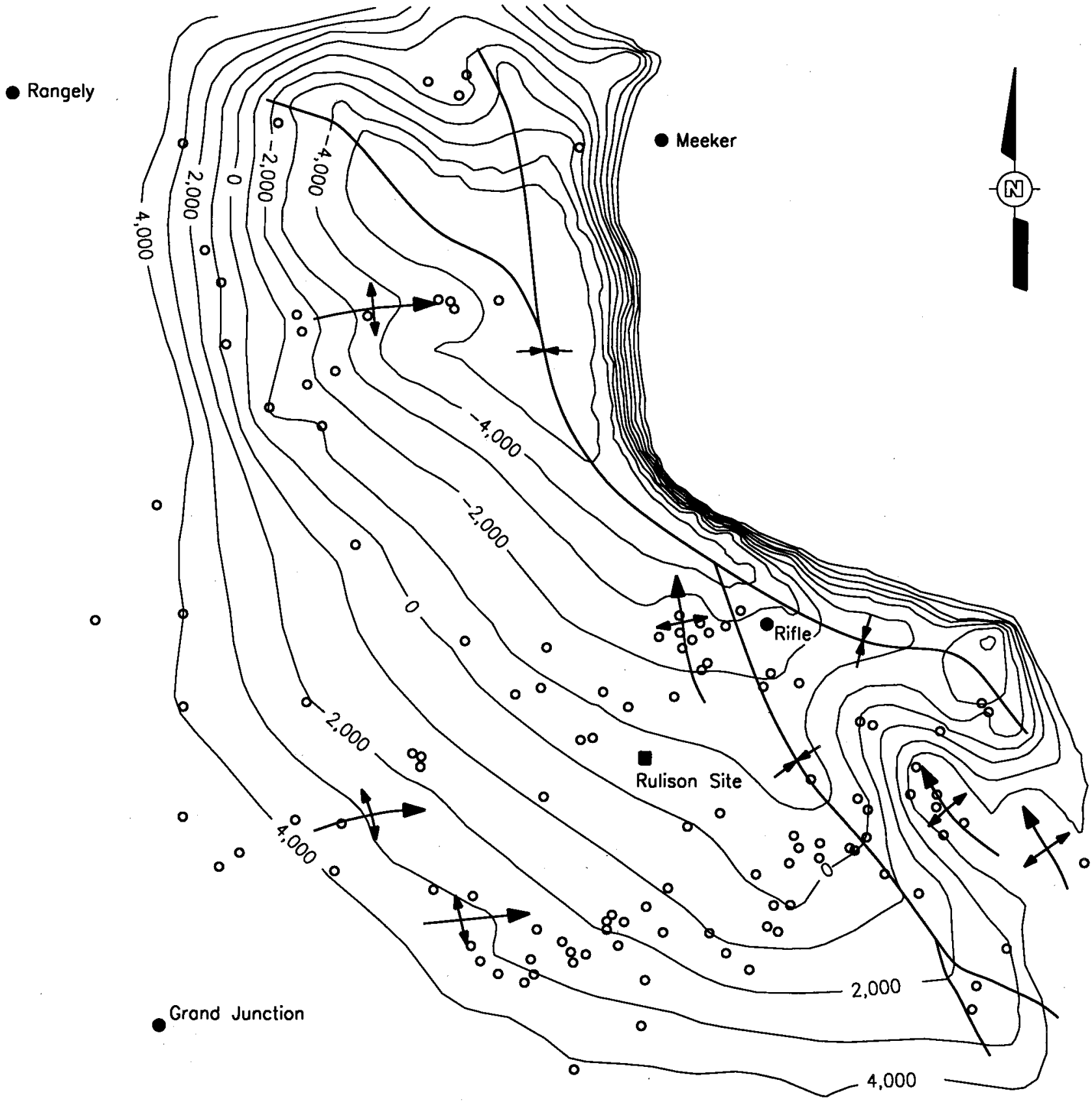

NOT TO SCALE

SCALE

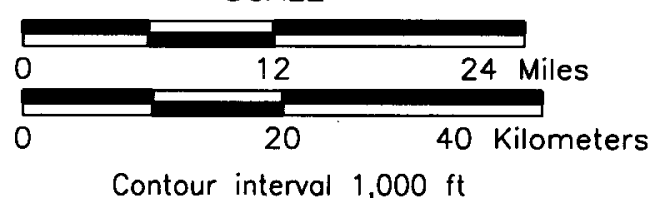

Source: Myal et al., 1987

\section{LEGEND}

- Well locations of structural dota

- City

- Rulison Site

Figure 5-8

Structure Contour Map of the Top of Marine Intervals (Rollins-Trout Creek) of the Piceance Basin, Colorado 
The Mesaverde Formation contains a tremendous gas resource throughout the Piceance Basin. However, because of its very low permeability, commercial development of the resource is often marginally economical. For this reason, the Rulison area has been the center of government- and institution-sponsored research to better understand Mesaverde production characteristics and enhancement potential. The Rulison test in 1969 in the Hayward 25-95 well was the first experiment to attempt to stimulate production of gas by fracturing the formation with a nuclear device.

\subsubsection{Gas Reservoir Characteristics}

The Mesaverde can be a prolific gas producer; however, it is often found to be "tight", having low porosity $(<10 \%)$ and low permeability ( $<0.05$ millidarcies) (CER, 1992). The highest production from the Mesaverde is limited to zones where natural, open fractures are encountered. When fractures are not encountered, fractures are artificially induced using hydraulic pressure (Hydrofracs). The enhanced or new fractures are then propped open using sands or other compounds. Artificially stimulated wells do not perform as well as wells that encounter natural open fractures (CER, 1989).

Based upon intensive analysis of the core, high resolution geophysical logging methods, and well interference tests, one dominant fracture set is present within the Mesaverde Group. These open fractures strike northwest-southeast parallel to the local basin axis (Figure 5-9). Wells that intersect these fractures show the highest rates of gas production (CER, 1989). Fracture development by artificial means tends to develop parallel to the dominant fracture set.

Gas produced from the Mesaverde is usually dry. However, water content within the reservoirs is variable, and water can be produced from the formation along with the gas.

\subsection{Surface Water}

\subsubsection{Streams, Springs, and Seeps}

There are three major surface water features at the Rulison Site. First, Battlement Creek is a rushing mountain stream that flows through the southwest corner of the site. Battlement Creek is principally fed by snow melt, shallow groundwater, and springs, and its flow is regulated upstream (south) of the site by Battlement Reservoir. Second, a smaller, spring-fed tributary of Battlement Creek flows across the site east of Battlement Creek. Third, an artificially created 


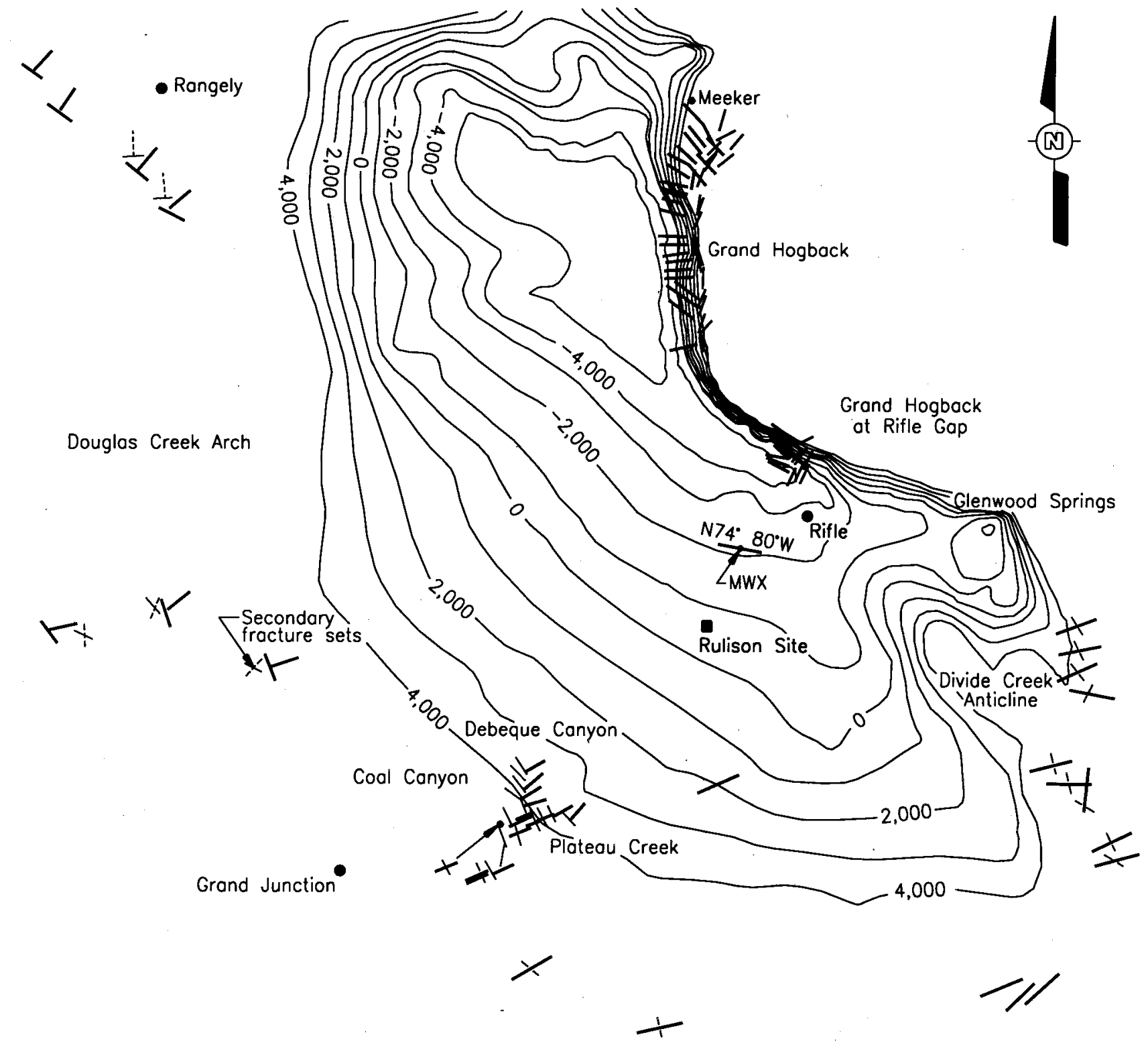

NOT TO SCALE

SCALE

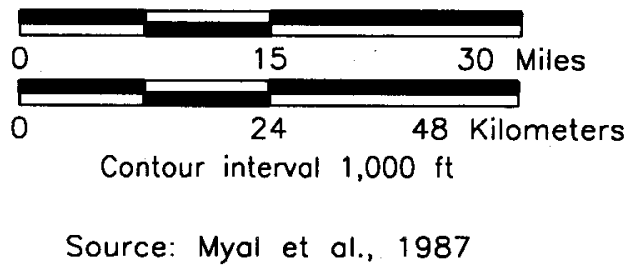

\section{LEGEND}

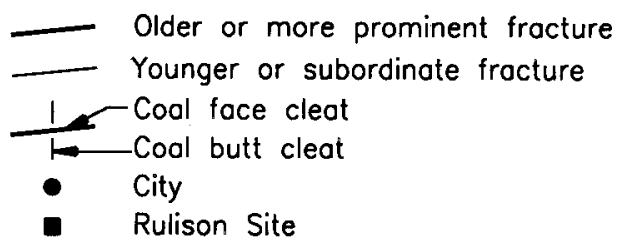

Figure 5-9

Compilation Map of the Fracture Orientation, Piceance Basin, Colorado 
drilling effluent pond is located at the center of the site. This pond was built to store drilling mud as part of emplacement hole drilling for the nuclear device.

Battlement Creek and its tributaries provide the main control over surface waters at the Rulison Site. The creek and the tributaries flow in a generally northwesterly direction toward the Colorado River.

An unnamed tributary (locally known as "Hayward" Creek) transects the Rulison Site and is adjacent to the effluent pond. Approximately $30 \mathrm{~m}$ (100 ft) below the effluent pond, this tributary flows into a series of beaver ponds (Figure 5-10). This stream is impounded by the beaver dams, creating a marshy, wetland complex through the middle of the site. Because of the topographic slope of the area, Battlement Creek and its tributaries are generally confined to relatively narrow stream channels except for the beaver pond area where the tributary channel widens because of the slower flow resulting from a more shallow stream gradient.

Additionally, several springs exist near the Rulison Site and the drilling effluent pond. The current source of water for the pond is from snow melt, groundwater, and a spring located approximately $300 \mathrm{~m}(915 \mathrm{ft})$ southeast of the pond, which replenishes the pond by surface flow via an inlet in the eastern berm. The pond also has an overflow in the western berm although the water level is seldom high enough for overflow to occur.

The Rulison drilling effluent pond is triangular in shape and covers approximately 1 acre. It is approximately 6-m (20-ft) deep (from top of the berm to pond bottom) and is located approximately $400 \mathrm{~m}(1,300 \mathrm{ft})$ north-northwest of SGZ. The pond originally was used for containment of surplus drilling fluids during the emplacement hole drilling operations. The pond is equipped with a spillway on the downslope side, $1.8 \mathrm{~m}(6 \mathrm{ft})$ below the crest. The present owner of the property, Lee Hayward, son of Claude V. Hayward, has retained the pond for his own use (AEC, 1973a, p. 5) and has converted the pond to a fresh-water trout pond. The pond is fenced to prevent access by wildlife and livestock. Because the effluent pond is an artificial impoundment that does not have the vegetative characteristics of a natural wetland, it has not been designated as a "wetland" (IT, 1993c, p. 4-1).

\subsubsection{Wetlands}

A wetlands, vegetation, and floodplains survey was conducted during June 1993 (IT, 1993c). An initial wetlands and floodplains determination for the Rulison Site was made using information 


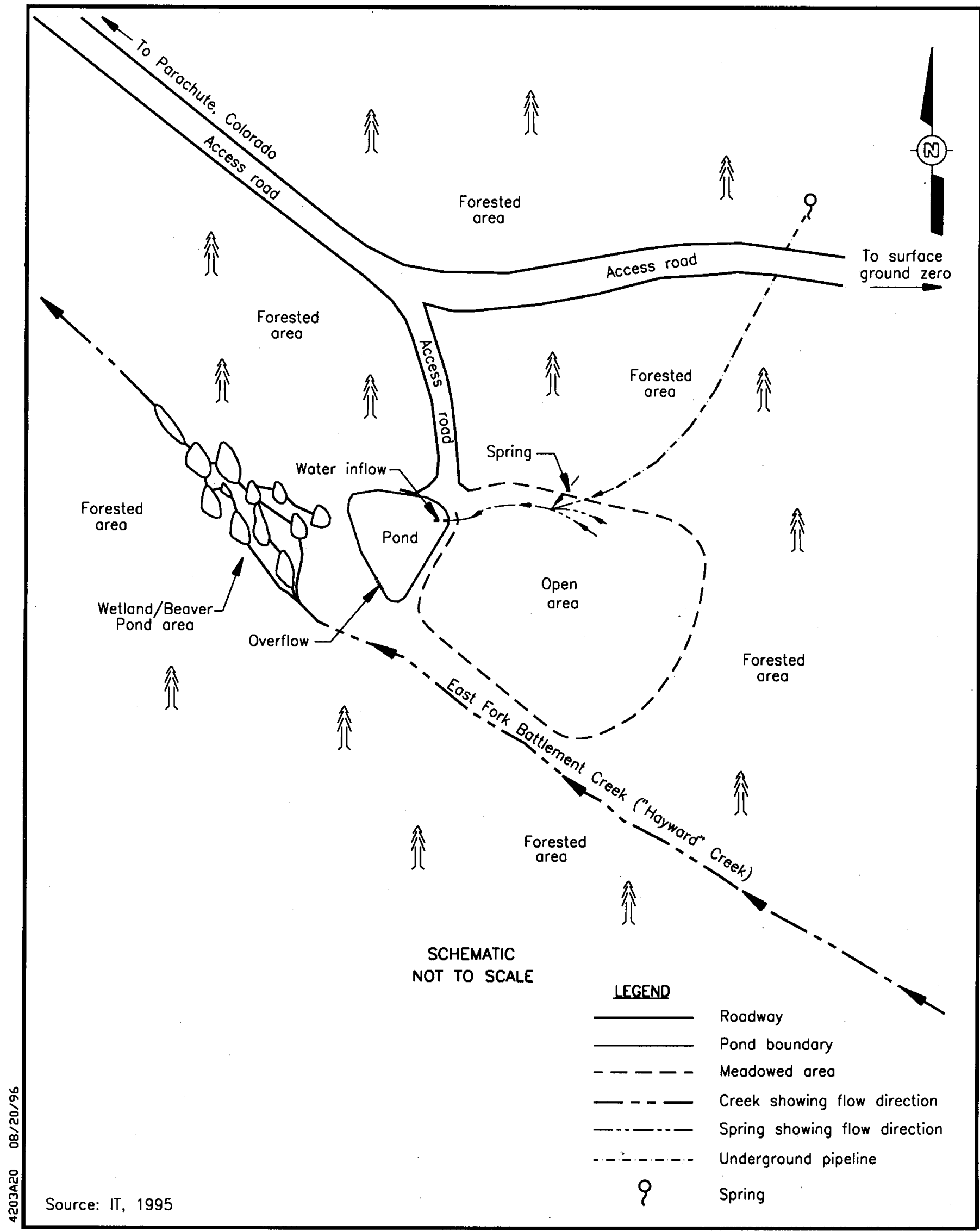

Figure 5-10

General Site Layout Diagram, Rulison Drilling Effluent Pond, January 1995 
from aerial photographs; a USGS topographic map (7.5 minute Rulison quadrangle); a Rifle Area, Colorado, Soil Survey (1980) map; and Flood Insurance Rate Maps (FIRM) for Garfield County, Colorado, in conjunction with field surveys.

Floodplains and wetlands were delineated using the methods outlined in the Corps of Engineers Wetlands Delineation Manual (U.S. Army Corps of Engineers, 1987), and the procedures outlined in Title 10 CFR Part 1022, "Compliance with Floodplains in Wetlands Environmental Review Requirements."

A list of dominant plant species found in upland and wetland communities at the Rulison Site is presented in Table 5-4.

The wetlands on the site are either associated with Battlement Creek or its tributary which transects the site. Battlement Creek flows within a narrow, well defined path. The high flow rate of Battlement Creek has scoured the channel, leaving a very rocky substrate supporting limited, if any, vegetation within the channel. However, the wooded slopes adjacent to the Creek contain a dense canopy of blue and Englermann spruce intermixed with quaking aspen. The understory contains mountain maple, water birch, and mountain alder.

The tributary to Battlement Creek, which transects the site, has a similar wetland community. These wetlands are due to adjacent springs feeding the tributary and beaver disturbance in the center of the site. The two most common species in this area are the quaking aspen and mountain maple in the canopy, with serviceberry and grasses in the understory and ground cover. Often, the aspen form pure stands. In the center of the site, beaver have removed the canopy layer and formed numerous ponds on several terraces. Associated with the terraces are saplings of quaking aspen with adult spruces intermixed. Sandbar willow is also common, recolonizing the wetter areas with common choke cherry sprouting in the drier areas. Numerous emergent species, such as grasses and sedges, were also observed colonizing the disturbed areas and on the beaver dams.

The center of the site also contains the man-made drilling effluent pond. This drilling effluent pond was created during the original testing activity on the site and is contained within an earthen berm that has little hydrophytic and no aquatic vegetation. 
Table 5-4

\section{List of Dominant Plant Species - Rulison Site Wetland Survey June 25 - 30, 1993}

\begin{tabular}{|c|c|c|c|}
\hline \multirow{3}{*}{$\begin{array}{c}\text { Scientific Name }^{\text {b }} \\
\text { Osmundaceae } \\
\text { Osmunda cinnamomea }\end{array}$} & \multirow{3}{*}{$\begin{array}{l}\text { Common Name } \\
\text { Cinnamon Fern }\end{array}$} & \multicolumn{2}{|c|}{ Indicator Status $^{\mathrm{a}}$} \\
\hline & & Regional & National \\
\hline & & NL & FACW \\
\hline $\begin{array}{l}\text { Gramineae } \\
\text { Gramineae spp. }\end{array}$ & Grasses & NIS & \\
\hline $\begin{array}{l}\text { Salicaceae } \\
\text { Salix exigua } \\
\text { Populus tremuloides }\end{array}$ & $\begin{array}{l}\text { Sandbar Willow } \\
\text { Quaking Aspen }\end{array}$ & $\begin{array}{l}\text { OBL } \\
\text { FAC }\end{array}$ & $\begin{array}{l}\text { FACW, OBL } \\
\text { FACU, FAC }\end{array}$ \\
\hline $\begin{array}{l}\text { Betulaceae } \\
\text { Betula occidentalis } \\
\text { Alnus tenuifolia }\end{array}$ & $\begin{array}{l}\text { Water Birch } \\
\text { Mountain Alder }\end{array}$ & $\begin{array}{l}\text { FACW } \\
\text { FACW }\end{array}$ & $\begin{array}{l}\text { FAC, FACW } \\
\text { FAC, FACW }\end{array}$ \\
\hline $\begin{array}{l}\text { Cyperaceae } \\
\text { Carex spp. }\end{array}$ & Sedge & NIS & FACW, OBL \\
\hline $\begin{array}{l}\text { Juncaceae } \\
\text { Juncus effusus }\end{array}$ & Soft Rush & $\mathrm{OBL}$ & FACW, OBL \\
\hline $\begin{array}{l}\text { Fagaceae } \\
\text { Quercus gambelii }\end{array}$ & Gamble Oak & $\mathrm{NL}$ & UPL \\
\hline $\begin{array}{l}\text { Rosaceae } \\
\text { Prunus virginiana } \\
\text { Amelanchier alnifolia } \\
\text { Cowania mexicana } \\
\text { Purshia tridentata }\end{array}$ & $\begin{array}{c}\text { Common Chokecherry } \\
\text { Western Serviceberry } \\
\text { Cliffrose } \\
\text { Antelope Brush }\end{array}$ & $\begin{array}{l}\text { FACU } \\
\text { FACU } \\
\text { UPL } \\
\text { UPL }\end{array}$ & $\begin{array}{l}\text { FACU, FAC } \\
\text { UPL, FAC } \\
\text { UPL } \\
\text { UPL }\end{array}$ \\
\hline $\begin{array}{l}\text { Aceraceae } \\
\text { Acer glabrum }\end{array}$ & Rocky Mountain Maple & FAC & FACU, FAC \\
\hline $\begin{array}{l}\text { Cornaceae } \\
\text { Cornus stolonifera }\end{array}$ & Red-Osier Dogwood & FACW & FAC, FACW \\
\hline $\begin{array}{l}\text { Pinaceae } \\
\text { Picea engelmannii } \\
\text { Picea pungens } \\
\text { Pinus edulis }\end{array}$ & $\begin{array}{l}\text { Engelmann Spruce } \\
\text { Blue Spruce } \\
\text { Colorado Pinyon }\end{array}$ & $\begin{array}{l}\text { FACU } \\
\text { FAC } \\
\text { UPL }\end{array}$ & $\begin{array}{l}\text { FAC, FACU } \\
\text { FAC } \\
\text { UPL }\end{array}$ \\
\hline $\begin{array}{l}\text { Typhaceae } \\
\text { Typha latifolia }\end{array}$ & Broad-Leaf Cattail & $\mathrm{OBL}$ & OBL \\
\hline $\begin{array}{l}\text { Balsaminaceae } \\
\text { Impatiens capensis }\end{array}$ & Jewelweed & FACW & FACW \\
\hline $\begin{array}{l}\text { Urticaceae } \\
\text { Urtica dioica }\end{array}$ & Stinging Nettle & FAC & FACU, FACW \\
\hline
\end{tabular}

Source: IT, 1993c

andicator status derived from the U.S. Fish and Wildlife Service's National List of Plant Species that occur in Wetlands:

1988 National Summary (Reed, 1988).

${ }^{b}$ Nomenclature conforms to that of Grays Manual of Botany (Fernald, 1950).

$\mathrm{OBL}=$ Obligate wetland plants that occur almost always in wetlands $(>99 \%)$

FACW = Facultative wetland plants that usually occur in wetlands (67-99\%)

FAC = Facultative plants that are equally likely to occur in wetlands or nonwetlands (34-66\%)

FACU $=$ Facultative upland plants that usually occur in nonwetlands $(1-33 \%)$

UPL = Obligate upland plants that occur almost always in nonwetlands (>99\%)

$\mathrm{NL} \quad=$ Species not listed

NIS $=$ Not identified to species 
Interviews with personnel who were present when the drilling effluent pond was constructed indicated that the pond may have been built on a spring or the pond may have been built below the local water table. Verbal reports by personnel who were present when the site was decommissioned indicate that groundwater entered the pond faster than it could be removed. In addition, the local surface expression of groundwater (springs) proximal to the Rulison Site indicates that the depth to groundwater may be less than expected based on regional information.

Finally, the pond-water level has remained stable after 26 years with only seasonal elevation changes observed, indicating that recharge to the pond and discharge from the pond have reached equilibrium with the local groundwater environment. The water level in the pond ranges from approximately 1 to $3 \mathrm{~m} \mathrm{(3} \mathrm{to} 10 \mathrm{ft}$ ) below the pond berm.

Based on this evidence and an inspection of the site hydrology (conducted on April 19 and 20, 1995), groundwater at the effluent pond is expected to be at a relatively shallow depth, following the natural topographic slope. At the south end of the pond, the water surface is anticipated to be equivalent to the groundwater surface. At the north end, the hydraulically down gradient end of the pond, the water surface is anticipated to be above the groundwater surface because of the damming action of the pond berm.

\subsubsection{Floodplains}

No flood plains or flood-prone areas have been identified at the Rulison Site based on review of the FIRM Index Map (FEMA, 1986) for Garfield County, Colorado, although a more detailed map has not been published.

\subsection{Hydrogeology}

\subsubsection{Occurrence of Groundwater}

The groundwater resources in the Rulison area are confined primarily to alluvium and surficial deposits (e.g., floodplain deposits and terrace and fan gravel). Essentially all the wells and most of the springs in the area derive their water from these shallow sources. Water in the alluvium occurs under both water-table and artesian conditions (Coffin et al., 1968, p. 8). Most of the springs are located along the contact of different strata within the surficial deposits. The underlying shale bedrock formations generally have low permeability and yield little or no water (Voegeli et al., 1970, p. 9).

Marine and nonmarine sedimentary rocks, approximately 5,486.40 m (18,000 ft) thick, underlie 
the Rulison Site. The emplacement (R-E) and exploratory (R-EX) holes, see Figure 5-11 (ERDA, 1977, p. 3), penetrated the following formations, in descending order:

- Quaternary alluvium is as much as $42.67 \mathrm{~m}(140 \mathrm{ft})$ thick;

- Green River Formation composed chiefly of shale and marlstone is about $518.16 \mathrm{~m}(1,700$ ft) thick;

- Wasatch Formation consisting principally of clay and shale with sandstone lenses is about $1,188.72 \mathrm{~m}(3,900 \mathrm{ft})$ thick;

- An unnamed unit of Paleocene age consisting of sandstone, shale, and a few thin beds of coal is about $152.40 \mathrm{~m}(500 \mathrm{ft})$ thick;

- $\quad$ Ohio Creek Conglomerate is about $11.28 \mathrm{~m}(37 \mathrm{ft})$ thick;

- Mesaverde Formation consisting mainly of sandstone and interbedded shale is about $762 \mathrm{~m}$ (2,500 ft) thick (Nork and Fenske, 1970, p. 5; Voegeli et al., 1970, pp. 5-7).

The Mesaverde Formation is of particular interest because the nuclear device was detonated within this group at a depth of 2,568.24 m (8,426 ft) in hole R-E (Voegeli, 1969, p. 4; Voegeli et al., 1970, p. 5; ERDA, 1977).

A small amount of water was found in an upper Mesaverde sandstone lens during the drilling of hole R-EX. Later tests of this zone and other zones thought to contain water in the Mesaverde yielded no significant groundwater. Several deep drill holes in the Ohio Creek Conglomerate above the Mesaverde Group in the Rulison gas field have produced water; hole R-EX produced no water from the Ohio Creek Conglomerate. The Wasatch Formation contains some sandy zones in the middle and the upper parts of the formation; however, these zones produced no water in hole R-EX. The lower Green River Formation, about 1,524 m (5,000 ft) above the detonation, has some sandy zones that produced water in sufficient quantities (none exceeding $0.73 \mathrm{~m}^{3} /$ day [4 gallons per minute]) to make air drilling difficult (Voegeli, 1969, p. 7; Voegeli et al., 1970, p. 15; DOE, 1984, p. 10). 


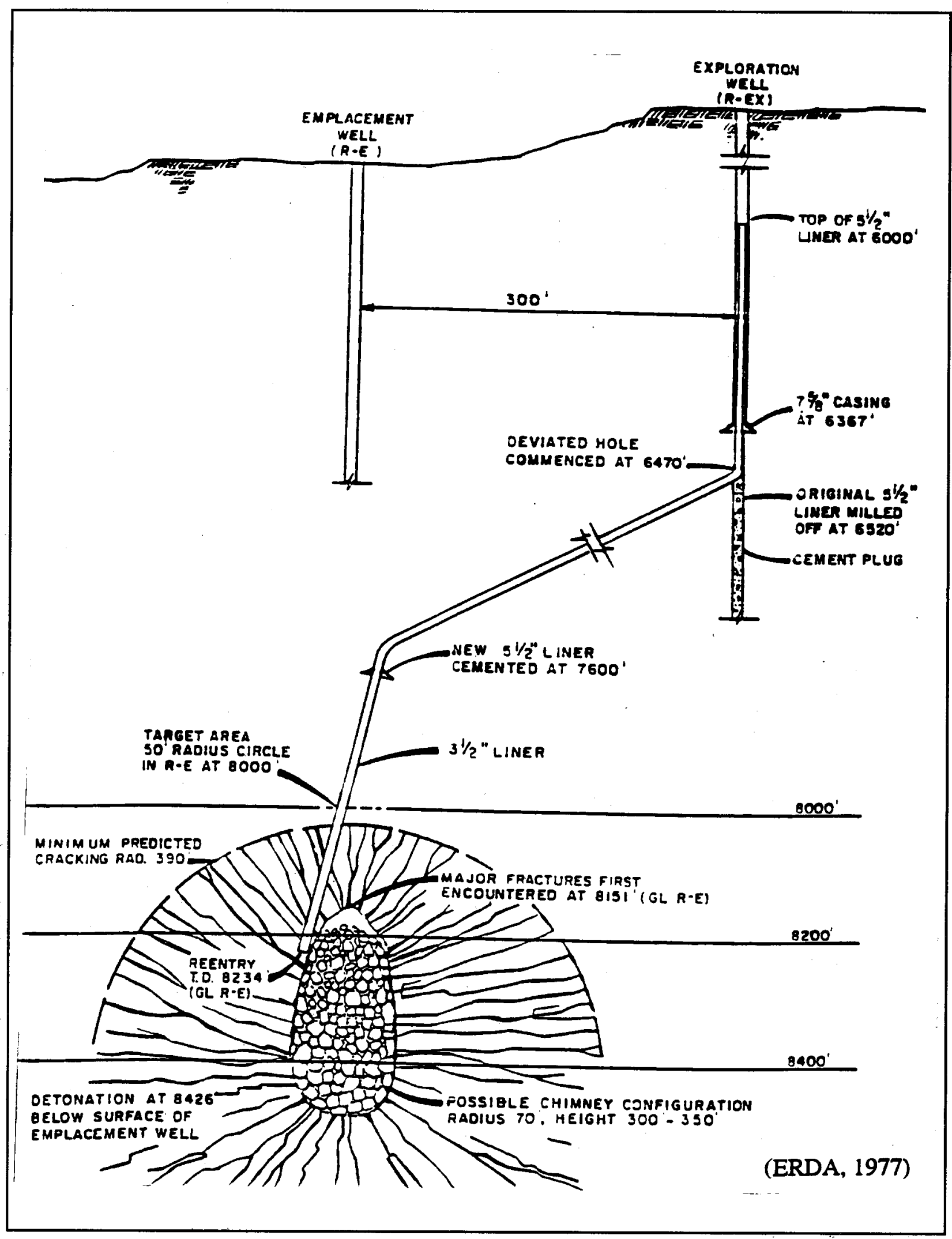

Figure 5-11

Project Rulison Emplacement and Reentry Well Configuration 
The Quaternary alluvial deposits are of particular importance since they provide most of the area's groundwater resources. The deposits include mudflows, talus accumulations, fan and pediment gravel, slump blocks, and the alluvium of Battlement Creek and the Colorado River. The regional water table ranges from 1.83 to $48.77 \mathrm{~m}$ (6 to $160 \mathrm{ft}$ ) below the land surface (Voegeli et al., 1970, pp. 25-28). The direction of groundwater flow in the alluvial deposits is expected to be northward, consistent with topographic slope. Rocks below the alluvium dip two degrees or less to the north and groundwater flow is expected to be northward also (Nork, 1969, p. 4; Voegeli et al., 1970, pp. 25-28; Nork and Fenske, 1970, p. 7).

\subsubsection{Hydraulic Characteristics}

Results of hydraulic tests in hole R-EX, shown in Table 5-5 (Voegeli et al., 1970, p. 19), indicate that samples consist primarily of drilling fluid rather than formation water. This suggests that the permeability of the formation is so low that little or no water movement occurs in the zones tested (Voegeli et al., 1970, p. 23). Although no fluid was recovered on any of the swab tests performed during the drill-stem tests, the complete absence of formation water cannot be ruled out as attested to by regular variations in other ions such as carbonate, sulfate, chloride, and sodium. The tritium content of the fluid indicates that it was derived from or contaminated by a surface source rather than from formation water (Voegeli, 1969, p. 14; Voegeli et al., 1970, p. 20).

Hydrologic tests were performed only in the Ohio Creek and Mesaverde rocks encountered in drill hole R-EX. Preshot permeability for the Mesaverde Formation was first estimated at 0.5 microdarcys $(\mu \mathrm{d})$ and then at $0.01 \mu \mathrm{d}$, while postshot production data and reservoir simulation studies indicated that actual matrix permeability was approximately 0.001 to $0.04 \mu \mathrm{d}$ (Stosur, 1977, p. 709). Additional porosities and permeabilities for deep rocks in the Rulison gas field are presented in DOE's Multi-Well Experiment reports (Sattler, 1984; Hart et al., 1984; CER, 1984; and Hart et al., 1987). Extensive pressure drawdown and build-up data for R-EX are reported by Austral and CER (1969, pp. I-1-IX-3).

Pressures recorded by the USGS during the testing of all water-bearing zones below the unnamed Paleocene unit indicate steep pressure build-up curves as a function of time, but yielded low fluid recoveries. This could indicate fracture dominated permeability. The presence of linear features on the land surface supports this theory. If there is fracture flow, lateral flow rates could be much higher than those previously predicted. The most permeable interval tested was from 2,193.34 to $2,193.95 \mathrm{~m}(7,196$ to $7,198 \mathrm{ft})$. The shut in pressure for this interval was 2,875 
Table 5-5

Summary of Hydraulic Tests, Hole R-EX

(Voegeli et al., 1970)

\begin{tabular}{|c|c|c|c|c|c|c|c|c|}
\hline $\begin{array}{l}\text { Geologic } \\
\text { Formation }\end{array}$ & $\begin{array}{l}\text { Depth of Zone } \\
\text { Tested Below } \\
\text { Land Surface } \\
\text { (feet) }\end{array}$ & $\begin{array}{l}\text { Date } \\
\text { Tested }\end{array}$ & $\begin{array}{c}\text { Casing } \\
\text { Size } \\
\text { (inches) }\end{array}$ & Perforations & $\begin{array}{l}\text { Type of } \\
\text { Test Tool }\end{array}$ & $\begin{array}{l}\text { Fluid Entry } \\
\text { During Time } \\
\text { Tool was Open }\end{array}$ & $\begin{array}{c}\text { Bottomhole } \\
\text { Temperature } \\
\left({ }^{\circ} \mathrm{F}\right)\end{array}$ & Remarks \\
\hline $\begin{array}{l}\text { Ohio Creek } \\
\text { Formation }\end{array}$ & 6,129 to 6,149 & $1-15-68$ & $75 / 8$ & $\begin{array}{l}3 / 8 \text { in. to } 1 / 2 \text { in. } \\
4 \text { per } \mathrm{ft}\end{array}$ & M.F.E. & $\begin{array}{l}\text { Pressure charts } \\
\text { indicated no fluid } \\
\text { entry. }\end{array}$ & 151 & $\begin{array}{l}\text { Recovered about } 15 \text { gallons of drilling } \\
\text { mud from top of test tool. }\end{array}$ \\
\hline Mesaverde Group & 7,066 to 7,080 & $4-8-68$ & $51 / 2$ & $\begin{array}{l}3 / 8 \text { in. to } 1 / 2 \text { in. } \\
2 \text { per } \mathrm{ft}\end{array}$ & F.A.S.T. ${ }^{2}$ & $\begin{array}{l}\text { Pressure charts } \\
\text { indicated no fluid } \\
\text { entry. }\end{array}$ & 196 & $\begin{array}{l}\text { Swabbed to } 7,004 \mathrm{ft} \text { below land } \\
\text { surface. No fluid recovered. } \\
\text { Recovered about } 10 \text { gallons of fluid } \\
\text { from top of test tool. } .^{3}\end{array}$ \\
\hline Mesaverde Group & 7,196 to 7,198 & $4-5 \& 6-68$ & $51 / 2$ & $\begin{array}{l}3 / 8 \text { in. to } 1 / 2 \text { in. } \\
2 \text { per } \mathrm{ft}\end{array}$ & F.A.S.T. ${ }^{2}$ & $\begin{array}{l}\text { Pressure charts } \\
\text { indicated no fluid } \\
\text { entry. }\end{array}$ & 195 & $\begin{array}{l}\text { Swabbed to } 7,134 \mathrm{ft} \text { below land } \\
\text { surface. No fluid recovered. } \\
\text { Recovered about } 240 \text { gallons of fluid } \\
\text { from top of test tool. }{ }^{3}\end{array}$ \\
\hline Mesaverde Group & 7,312 to 7,320 & $4-4 \& 5-68$ & $51 / 2$ & $\begin{array}{l}3 / 8 \text { in. to } 1 / 2 \text { in. } \\
2 \text { per ft }\end{array}$ & F.A.S.T. ${ }^{2}$ & $\begin{array}{l}\text { Pressure charts } \\
\text { indicated no fluid } \\
\text { entry. }\end{array}$ & 196 & $\begin{array}{l}\text { Swabbed to } 7,250 \mathrm{ft} \text { below land } \\
\text { surface. No fluid recovered. } \\
\text { Recovered about } 15 \text { gallons of fluid } \\
\text { from top of test tool. } .^{3}\end{array}$ \\
\hline Mesaverde Group & 7,598 to 7,604 & $4-3 \& 4-68$ & $51 / 2$ & $\begin{array}{l}3 / 8 \text { in. to } 1 / 2 \text { in. } \\
2 \text { per } \mathrm{ft}\end{array}$ & F.A.S.T. ${ }^{2}$ & $\begin{array}{l}\text { Pressure charts } \\
\text { indicated no fluid } \\
\text { entry. }\end{array}$ & 197 & $\begin{array}{l}\text { Swabbed to } 7,544 \mathrm{ft} \text { below land } \\
\text { surface. No fluid recovered. } \\
\text { Recovered about } 20 \text { gallons of fluid } \\
\text { from top of test tool. } .^{3}\end{array}$ \\
\hline Mesaverde Group & 8,014 to 8,018 & $3-28-68$ & $51 / 2$ & $\begin{array}{l}3 / 8 \text { in. to } 1 / 2 \text { in. } \\
2 \text { per ft }\end{array}$ & F.A.S.T. ${ }^{2}$ & $\begin{array}{l}\text { Pressure charts } \\
\text { indicated no fluid } \\
\text { entry. }\end{array}$ & 199 & $\begin{array}{l}\text { Swabbed to } 7,929 \mathrm{ft} \text { below land } \\
\text { surface. No fluid recovered. } \\
\text { Recovered about } 30 \text { gallons of fluid } \\
\text { from top of test tool. }{ }^{3}\end{array}$ \\
\hline
\end{tabular}

${ }_{2}^{1}$ Johnston Testers Multi-Flow Evaluator.

2 Johnston Testers Fracturing Acidizing Squeezing Tool.

${ }^{3}$ Fluid likely to have entered the tubing after the packer was pulled loose. 
pounds per square inch, which is adequate to support a column of water 2,020.82 $\mathrm{m}(6,630 \mathrm{ft})$ high or $172.52 \mathrm{~m}$ (566 ft) below land surface (Voegeli et al., 1970; Nork and Fenske, 1970, p. 6).

Little information was obtained about the hydraulic properties of the rocks above $1,828.80 \mathrm{~m}$ (6,000 ft) (Nork, 1969, p. 4; Nork and Fenske, 1970, p. 5). However, water-bearing characteristics for the same geologic formations in the shallow groundwater aquifer system slightly north $(<48.27 \mathrm{~km}[<30 \mathrm{mi}])$ of the Rulison Site, presented in Table 5-6 (Coffin et al., 1968 , p. 3), are assumed to be representative of the water-bearing characteristics for the alluvium and Green River Formation in the Rulison area.

The transmissibility of the alluvial fill differs from place to place. In places where the alluvium is mainly sand and gravel, transmissibility may be as much as 1,242.08 $\mathrm{m}^{2} /$ day (100,000 gallons per day [gpd]/ft). In places where the alluvium contains clay beds, the transmissibility may be as low as $248.42 \mathrm{~m}^{2} /$ day $(20,000 \mathrm{gpd} / \mathrm{ft})$. The average coefficient of storage probably averages about 0.20 (Coffin et al., 1968, p. 17). Thus, well yields depend largely on the lithology of the alluvium at the well, and the location of the well with respect to local hydrologic boundaries.

Specific conductance of the water decreased from about 12,000 to 10,000 microhoms, which may indicate a layering of the water and subsequent mixing when pumped (Coffin et al., 1968, p. 17).

Results of pumping and recovery tests in the Green River Formation indicate a range of transmissibility from 12.42 to $24.84 \mathrm{~m}^{2} /$ day (1,000 to 2,000 gpd/ft) (Coffin et al., 1968, pp. 17-18) and a storage coefficient of $1 \times 10^{-5}$ (Coffin et al., 1968, p. 21).

\subsubsection{Regional Hydrochemistry}

A pre-shot inventory of wells and springs in the Rulison area was conducted by the USGS between March 20 and May 25, 1969, to document the condition of wells and springs and to collect water samples for chemical and radiochemical analysis. All known wells within a 9.65-km (6-mi) radius of the Rulison emplacement hole, as well as selected wells and springs within a 16.09- to 32.18-km (10- to 20-mi) radius, are given in Appendix B (Hurr et al., 1969, pp. 3-9; Voegeli et al., 1970, pp. 25-31). Figure 5-12 shows the location of the water-sampling points in the network (Claassen, 1971, p. 3). Detailed location descriptions of sampling sites are presented in Voegeli et al. (1970, pp. 35-37). 


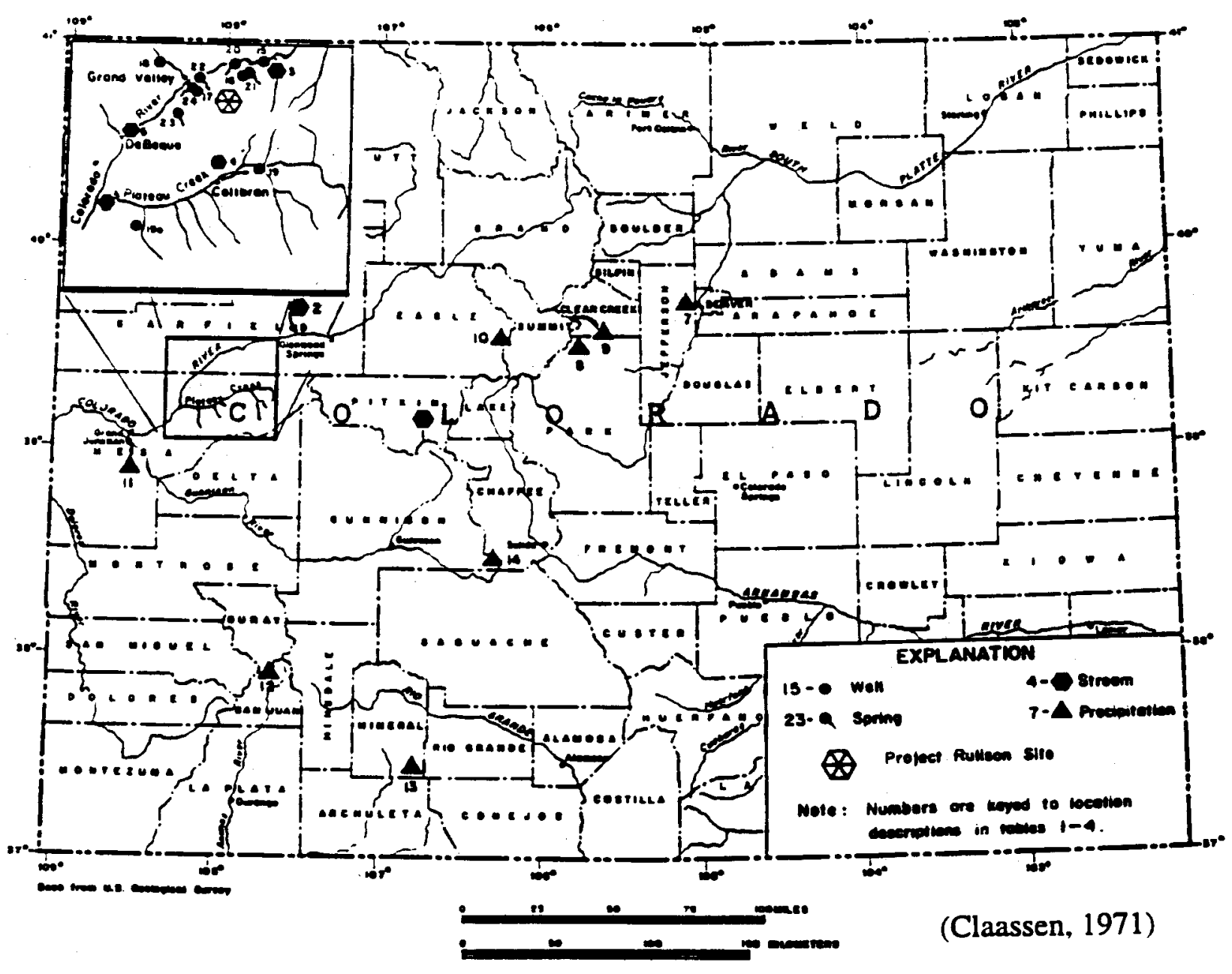

Figure 5-12

Location of the U.S. Geological Survey Water-Sampling Points, Project Rulison 
Table 5-6

Summary of the Water-Bearing Characteristics of the Geologic Formation

(Coffin et al., 1968)

\begin{tabular}{|c|c|c|c|c|c|c|c|c|}
\hline system & series & & Geulugie unit & $\begin{array}{l}\text { Thicinneas: } \\
\text { (fcot) }\end{array}$ & Phystcal character & water quality & Hydrologic charecter & mator supply \\
\hline 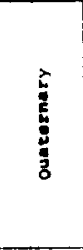 & 惫 & & Alluviun & $0-140$ & 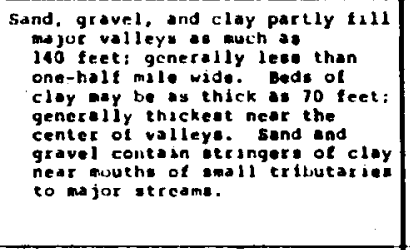 & 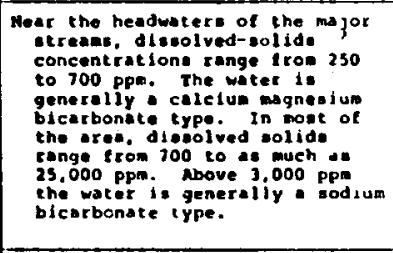 & 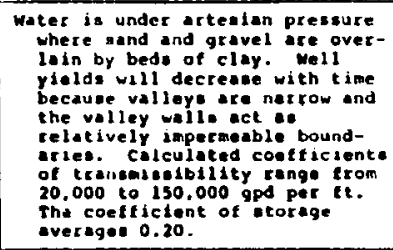 & 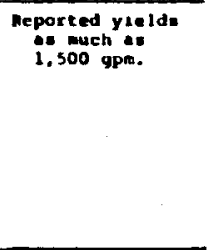 \\
\hline \multirow{6}{*}{ 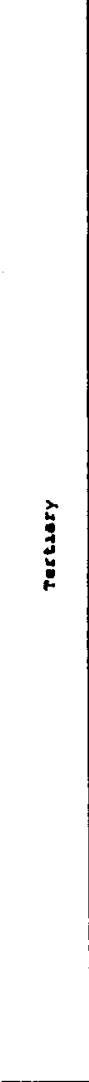 } & \multirow{6}{*}{ : } & \multirow{5}{*}{ 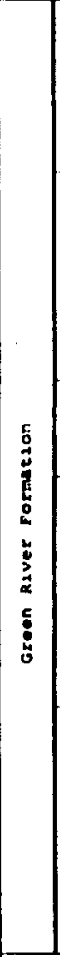 } & $\begin{array}{l}\text { Evacuation creck } \\
\text { Mendie: }\end{array}$ & $0-1,250$ & 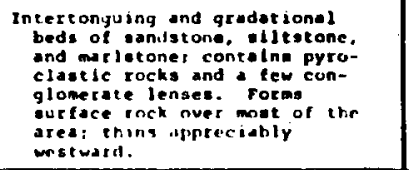 & 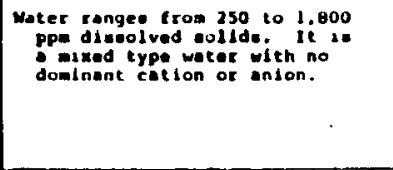 & 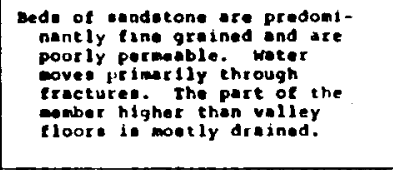 & 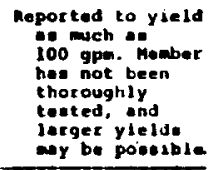 \\
\hline & & & $\begin{array}{l}\text { Parschulu Crarkh } \\
\text { Member }\end{array}$ & $500-1$, , 000 & 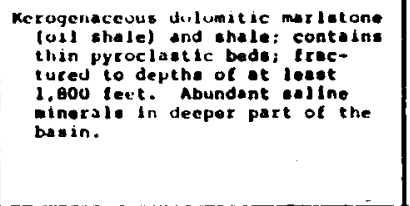 & 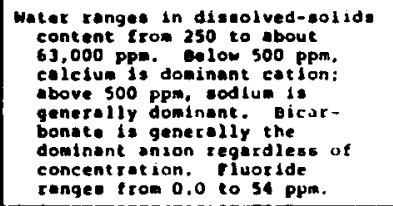 & 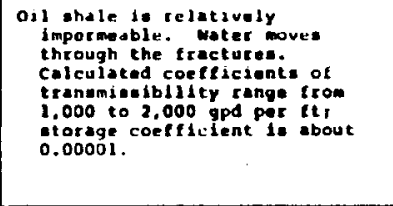 & 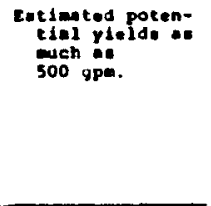 \\
\hline & & & $\begin{array}{l}\text { Garden Gulch } \\
\text { memiser }\end{array}$ & $0-900$ & 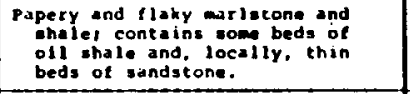 & 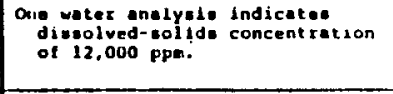 & $\begin{array}{l}\text { Keletively impermasble and } \\
\text { probaly conteine feu } \\
\text { traturas. }\end{array}$ & $\begin{array}{l}\text { Mot known to } \\
\text { yleld water to } \\
\text { wolle. }\end{array}$ \\
\hline & & & $\begin{array}{l}\text { Desuglax creek } \\
\text { Member }\end{array}$ & 0.800 & 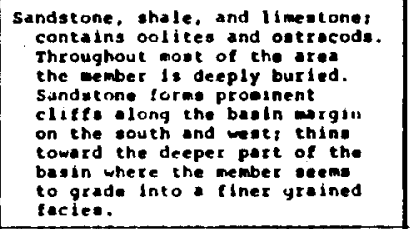 & 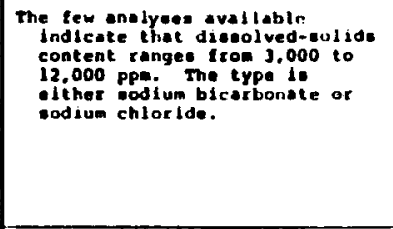 & $\begin{array}{l}\text { Reletively low permability } \\
\text { and probably little } \\
\text { fractured. }\end{array}$ & 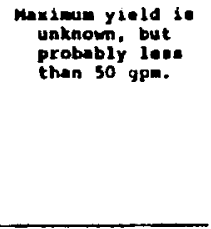 \\
\hline & & & $\begin{array}{l}\text { Anvili point: } \\
\text { Mamber }\end{array}$ & $0-1.870$ & 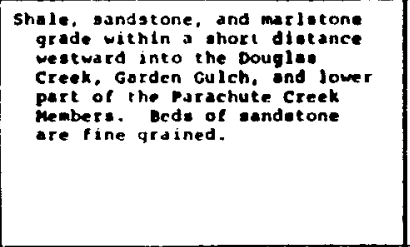 & 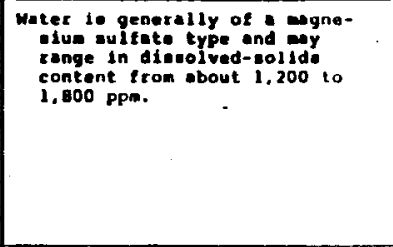 & $\begin{array}{l}\text { Dede of andatone are poorly } \\
\text { permasbla. }\end{array}$ & 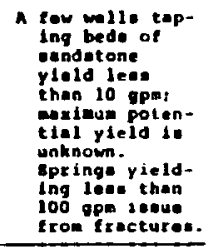 \\
\hline & & \multicolumn{2}{|r|}{ Wastlech For nat zun } & $300-5,000$ & 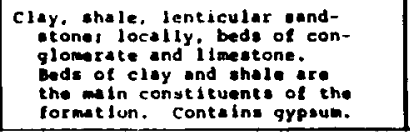 & 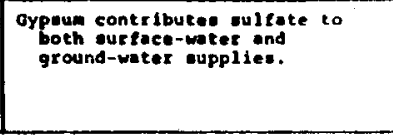 & 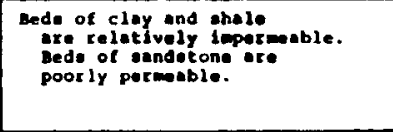 & $\begin{array}{l}\text { Mot known to } \\
\text { yiold water to } \\
\text { weile. }\end{array}$ \\
\hline
\end{tabular}


The results of chemical analyses of groundwater (Hurr et al., 1969, pp. 12-13; Larson and Beetem, 1970, pp. 14-15; Voegeli et al., 1970, pp. 32-33) and surface water samples (Larson and Beetem, 1970, p. 8; Voegeli et al., 1970, p. 37) are given in Appendix C. The results of radiochemical analyses of spring and well samples collected during re-entry drilling at the Rulison Site (Voegeli and Claassen, 1971a, pp. 13-14; Voegeli and Claassen, 1971b, pp. 7-8), as well as radiochemical data obtained from stream samples in the Rulison area (Voegeli and Claassen, 1971a, p. 12; Voegeli and Claassen, 1971b, p. 6; Claassen and Voegeli, 1971, p. 4; Claassen, 1971, p. 4), are presented in Appendix D.

Tritium results are given in Appendix A (DOE, 1984, p. 24). Background levels of tritium in surface waters averaged $910+/-570$ picoCuries per liter $(\mathrm{pCi} / \mathrm{L})$, well water samples averaged 640 +/- $450 \mathrm{pCi} / \mathrm{L}$, and spring water samples averaged 770 +/- $770 \mathrm{pCi} / \mathrm{L}$. Water samples collected during flaring ranged from less than 400 to 1,600 pCi/L (Boysen, 1976, p. 31). Numerous analytical results of water samples, as well as environmental and biological samples, collected from the Rulison area are given in Boysen, (1976). Atmospheric levels of radiation, as well as radiation exposures to off-site populations, are reported by the EPA (1974).

Prior to the completion of site cleanup, water samples were collected from two springs at the site, one located just off the southeast corner of the R-EX well pad and the other on the upper side of the road about $274.32 \mathrm{~m}$ (300 yards) downhill from the pad. No radioisotopes other than those naturally occurring were detected (AEC, 1973, p. 12; Eberline, 1977, p. 5). Decontamination of drilling equipment and radioactive fallout from gas flaring operations are also possible sources of shallow aquifer contamination. Extensive soil sampling at the site was done to access surface contamination resulting from radioactive fallout during gas flaring. Contaminated soil was removed from the site and transported to a suitable disposal site (Eberline, 1977).

Source term concentrations were estimated by assuming that the radionuclides are completely and uniformly mixed with a quantity of water equivalent to the volume of the cavity void space anticipated to be formed by the detonation. Predictions of cavity dimensions are given in Table 57 (AEC, 1969, p. 1; Nork and Fenske, 1970, p. 8). The cavity volume is calculated to be about 56,640 to $141,600 \mathrm{~m}^{3}\left(2 \times 10^{6}\right.$ to $5 \times 10^{6}$ cubic feet $)$. In this water volume, tritium concentration would be about $6 \times 10^{-3}$ microCuries per milliliter $(\mu \mathrm{Ci} / \mathrm{mL})$ to $2 \times 10^{-1} \mu \mathrm{Ci} / \mathrm{mL}$ (Nork, 1969, p. 5); strontium-90 concentration would be about $4 \times 10^{-2} \mu \mathrm{Ci} / \mathrm{mL}$ to $1 \times 10^{-1} \mu \mathrm{Ci} / \mathrm{mL}$ (Nork and Fenske, 1970, p. 11). From the post-shot drilling data, it was estimated that the rubble-filled chimney was approximately $106.68 \mathrm{~m}(350 \mathrm{ft})$ in height. This is greater than the 
Table 5-7

Physical Explosion Effects

\begin{tabular}{||l|c|c|c|c||}
\hline & Maximum & Mean & Minimum & Units \\
\hline \hline Cavity Radius & 108 & 90 & 72 & feet \\
\hline Cracking Radius & 580 & 485 & 390 & feet \\
\hline Chimney Height & 451 & 376 & 301 & feet \\
\hline $\begin{array}{l}\text { Cavity Volume (or } \\
\text { Chimney Void Space) }\end{array}$ & $5.28 \times 10^{6}$ & $3.05 \times 10^{6}$ & $1.56 \times 10^{6}$ & cubic feet \\
\hline Chimney Volume & $16.5 \times 10^{6}$ & $9.57 \times 10^{6}$ & $4.90 \times 10^{6}$ & cubic feet \\
\hline
\end{tabular}

Source: Nork and Fenske, 1970

minimum $91.74 \mathrm{~m}(301 \mathrm{ft})$ that was predicted, but comparable with the associated cavity radii dimensions determined from well test data (Reynolds, 1971, p. 1).

\subsubsection{Regional Flow System}

The Rulison Site is on the southwest limb of the Piceance Creek basin, a large northwest- trending structural downwarp in northwestern Colorado (Figure 5-13). The northern part of the Piceance Creek basin drains to the White River; the southern part of the basin drains to the Colorado River. The Rulison Site drains northward to the Colorado River (Voegeli, 1969, p. 4; Voegeli et al., 1970, p. 4).

The principal surface hydrologic feature of the Rulison Site is Battlement Creek, a stream that discharges to the Colorado River at Parachute, Colorado. Battlement Creek carries most of the runoff to the river, while some runoff is diverted for irrigation use and some infiltrates the stream alluvium and terrace deposits. The underflow in the alluvium appears as springs in several places downstream from the Rulison Site (Voegeli, 1969, p. 7; Voegeli et al., 1970, p. 7). Ranchers on Morrisania Mesa obtain water for their domestic and livestock usage from shallow wells in alluvium and terrace deposits or from cisterns and ponds which obtain their water from Battlement Creek and other small streams and springs (Voegeli, 1969, p. 10; Voegeli et al., 1970, p. 5). Municipal groundwater resources in the Rulison area are confined primarily to alluvium and surficial deposits (e.g., flood-plain deposits and terrace and fan gravel) (Voegeli, 1969, p.7). 


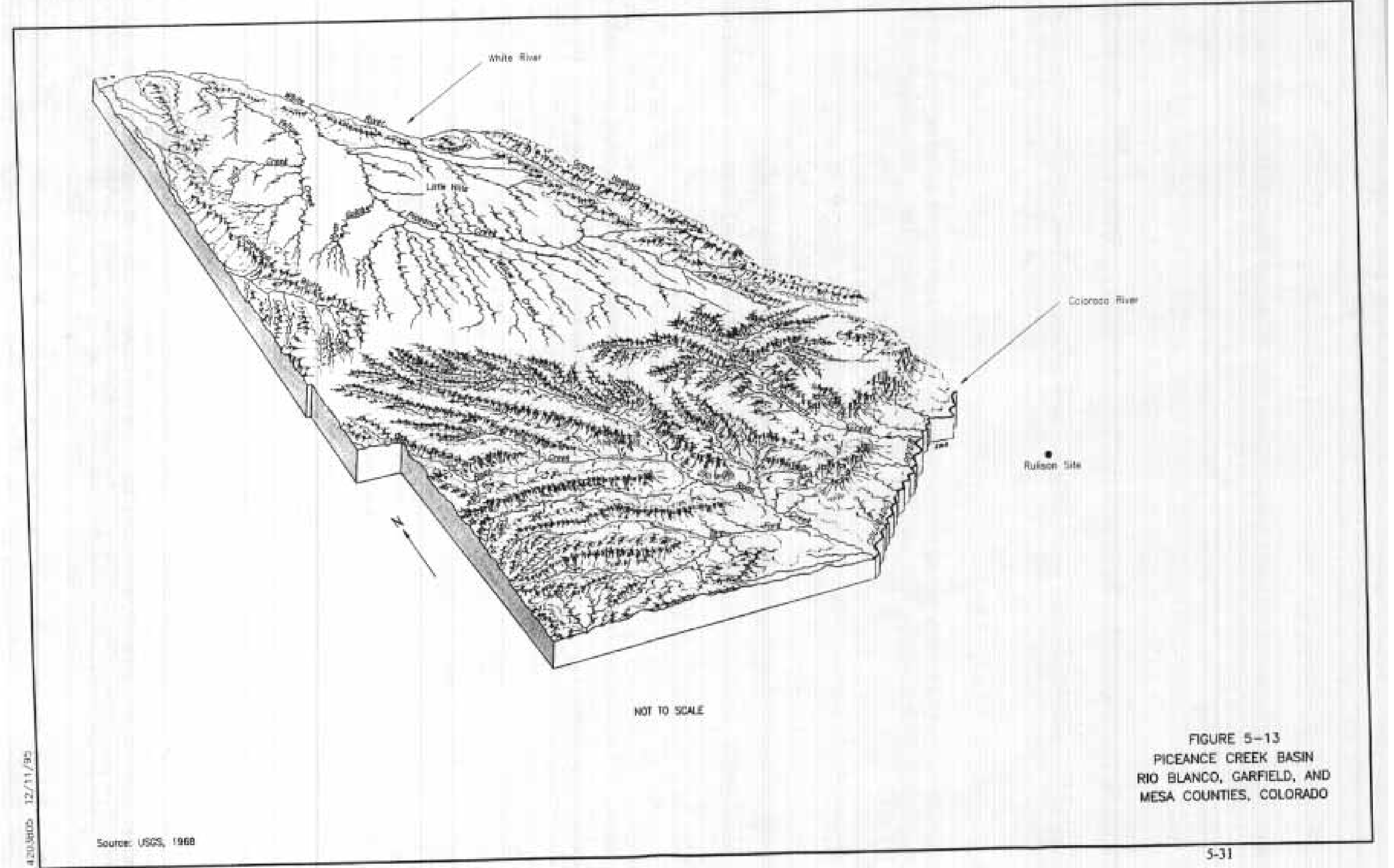




\subsubsection{Impact of Test on Hydrology}

Studies of pre-shot and postshot hydrologic conditions indicate that the detonation had no effect on the physical, chemical, or radiochemical characteristics of wells, springs, streams, shallow aquifers, or reservoirs in or near the Rulison Site (Voegeli et al., 1970, p. 48; AEC, 1973, p. 18). The USGS also sampled springs, rivers, and wells before and after reentry drilling and after each of the three gas production tests with the same negative results (DOE, 1984, pp. 15-16).

The Rulison device was emplaced near the base of the Mesaverde Formation at a depth of $2,568 \mathrm{~m}(8,426 \mathrm{ft})$. Essentially all of the explosion-produced radionuclides were contained within the Mesaverde Formation. Any mobile water in the Mesaverde Formation which becomes contaminated with explosion nuclides, and is located below about 2,133.60 m (7,000 ft), is expected to move downward or laterally, but not upward. Above 2,133.60 m (7,000 ft), any contaminated mobile waters are expected to move laterally. Groundwater movement in this formation is estimated to be a maximum of $0.3048 \mathrm{~m}(1 \mathrm{ft})$ per day. The most probable rate is essentially negligible (Nork and Fenske, 1970, p. 2).

Six drill stem tests were run in the vicinity of the shot point. The USGS interpreted the chemical character of fluids collected from tubing after each drill stem test in exploration hole R-EX as indicating that "little mobile water occurs in the zones tested" (Voegeli, 1969, P.14). Three of these tests, $2,153.72$ to $2,157.98 ; 2,193.34$ to $2,193.95$; and 2,228.70 to $2,231.14 \mathrm{~m}$ (7,066 to 7,$080 ; 7,196$ to 7,198; and 7,312 to 7,320 ft) below land surface resulted in pressure build-up curves that could be extrapolated to infinite time by the Van Everdingen method to estimate the virgin aquifer pressures. Table 5-8 shows the extrapolated shut-in pressures along with the post shot reservoir pressure compared to estimated hydrostatic pressures for the same depths.

The actual distribution of pressures above 7,066 feet are not well known. However, there can be no general upward or downward movement of water in this interval, and lateral flow must predominate. Below 7,066 feet pressures drop off rapidly and downward movement of water is expected to a point within or below the 7,312 to 7,320 foot interval. Since the pressure increases below this interval, a drain exists between 7,312 and about 8,442 feet where lateral flow is likely.

The three drill stem tests analyzed indicate relatively steep pressure build-up curves as a function of time but low fluid recoveries. A possible explanation of this phenomenon is that the predominant permeability belongs to a fracture system. The presence of many linears on the geologic map at the Rulison Site tends to substantiate this hypothesis. If this is the case, lateral 


\section{Table 5-8}

\section{R-EX Drill Stem Test Formation Pressures}

\begin{tabular}{||c|c|c||}
\hline $\begin{array}{c}\text { Depth } \\
\text { (feet) }\end{array}$ & $\begin{array}{c}\text { Estimated Shut-In Pressure } \\
\text { (pounds per inch) }\end{array}$ & $\begin{array}{c}\text { Estimated Hydrostatic Pressure } \\
\text { (pounds per inch) }\end{array}$ \\
\hline \hline $7,066-7,080$ & 3,050 & 3,050 \\
\hline $7,196-7,198$ & 2,900 & 3,096 \\
\hline $7,312-7,320$ & 2,250 & 3,150 \\
\hline$\approx 8,442$ & 2,950 & 3,640 \\
\hline
\end{tabular}

flow of water could occur at significant velocities in terms of usual groundwater flow rates. However, since the interfracture blocks in the sandstone beds must also have some permeability, all water would also have to flow through these low permeability blocks. The average water velocity is therefore expected to be extremely low (Nork and Fenske, 1970, pp. 5-6).

If groundwater in the Mesaverde Formation is immobile, all radioactivity will reside essentially in place until artificially removed, and will eventually decay below detection levels. If the groundwater in the Mesaverde Formation is mobile, very likely the velocity of movement will be slow enough and chemical-exchange retardation high enough to prevent the transport of radionuclides in greater-than-contaminant guideline (CG) concentrations for any significant distances. Although distribution coefficient distribution coefficient $\left(\mathrm{K}_{\mathrm{d}}\right)$ values were not determined for the Rulison Site, approximation for retardation of radionuclides may be determined using values from other locations, given in Table 5-9 (Nork, 1969, p. 7; Nork and Fenske, 1970, p. 13). Assuming a $0.31 \mathrm{~m} /$ day ( $1 \mathrm{ft} /$ day) rate of flow, it is predicted that tritium would move less than $1.61 \mathrm{~km}(1 \mathrm{mi})$ before decaying to a concentration less than $1 \times 10^{6} \mathrm{pCi} / \mathrm{L}$ (AEC, 1973, p. 18; DOE, 1984, p. 14). Under the same conditions of movement but with consideration of retardation effects (assuming $\mathrm{K}_{\mathrm{d}}=10$ ), strontium-90 would probably move less than $1.62 \mathrm{~km}$ (1 mi) before decay to below one CG (Nork, 1969, p. 8; Nork and Fenske, 1970, p. 14).

It is not clear what contaminant release scenario or scenarios were considered in the selection of Long-Term Hydrologic Monitoring Program (LTHMP) sampling sites (refer to Figure 2-11). It appears that rather than drilling a network of monitoring wells based on hydrologic data, the 
Table 5-9

Distribution Coefficients of Strontium-85 and

Cesium-137 for Various Materials

(Material suspended in 4 parts saturating solution for 72 hours.

Minimum particle diameter is 4,000 $\mu$ [Nork, 1969].)

\begin{tabular}{|c|c|c|c|}
\hline \multirow[t]{2}{*}{ Material } & \multirow[t]{2}{*}{$\begin{array}{c}\text { Saturating } \\
\text { Medium } \\
\end{array}$} & \multicolumn{2}{|c|}{$\mathrm{Kd}(\mathrm{Ml} / \mathrm{g})$} \\
\hline & & $\underline{\mathbf{S r}}$ & $\mathrm{Cg}$ \\
\hline $\begin{array}{l}\text { Basalt } \\
\text { (Amchitka) }\end{array}$ & Sea Water & 1.07 & 6.50 \\
\hline $\begin{array}{l}\text { Carbonate } \\
\text { (Yucca Flat, } \\
\text { Nevada Test Site) }\end{array}$ & $\begin{array}{l}\text { Prepared Water* } \\
\quad(\text { Well) }\end{array}$ & 0.19 & 13.5 \\
\hline $\begin{array}{l}\text { Salt } \\
\text { (Tatum Salt Dome) }\end{array}$ & Salt Saturated Water & 0.19 & 0.02 \\
\hline $\begin{array}{l}\text { Shaley Siltstone } \\
\text { (Gasbuggy Site, } \\
\text { Northern New Mexico) }\end{array}$ & GB-2 Well Water & $\begin{array}{r}8.32 \\
+\quad\end{array}$ & 309 \\
\hline $\begin{array}{l}\text { Sandstone } \\
\text { (Gasbuggy Site, } \\
\text { Northern New Mexico) }\end{array}$ & GB-2 Well Water & 1.37 & 102 . \\
\hline $\begin{array}{l}\text { Granite } \\
\text { (Shoal Site, Nevada) }\end{array}$ & Deep Formation Water & 1.7 & 34.3 \\
\hline $\begin{array}{l}\text { Tuff } \\
\text { (Rainier Mesa, } \\
\text { Nevada Test Site) }\end{array}$ & $\begin{array}{l}\text { Prepared Water* } \\
\text { (Rainier Spring) }\end{array}$ & 260 . & 1020 . \\
\hline $\begin{array}{l}\text { Desert Alluvium } \\
\text { (Hot Creek Valley, Nevada) }\end{array}$ & "Deep Formation Water & $50-2450$ & $70-2640$ \\
\hline
\end{tabular}

*Water prepared to have major chemical composition similar to that of referenced water source. 
LTHMP groundwater sampling program has clearly focused on local domestic supply wells and springs already in place as discussed in Section 2.2.2.4.2 Second Sampling Program, Water Sampling (Chapman and Hokett, 1991, p. 36).

The alluvial deposits are separated from the emplacement horizon by great thicknesses of low permeability formations, making transport of contaminants through the geologic media unlikely. The most probable mechanism for contaminant transport to the shallow monitoring wells from the shot point at a depth of over 2,438.40 $\mathrm{m}(8,000 \mathrm{ft})$ involves contaminant transport up the test holes. However, the presence of a low-pressure horizon at a depth of about 2,194.56 m (7,200 $\mathrm{ft}$ ) is presumed to behave as a sump between the shot depth and near-surface aquifers. This zone will prevent vertical flow into the higher pressure zones above, diverting contaminants to lateral flow along this hydrologic drain (Voegeli et al., 1970). In addition, the boreholes were plugged. The possibility of surface contamination by fallout during gas flaring operations was addressed by monitoring during flaring and presumably no longer poses a threat (Chapman and Hokett, 1991, p. 36).

Clearly, if the borehole release scenario is verifiably impossible, there is no reason to monitor the quality of the shallow aquifer. However, given that it is the only scenario proposed that could result in contamination of local supply aquifers, the LTHMP at Rulison is evaluated on the basis that contaminant transport is only possible through the boreholes drilled for the test.

During September 1995, DOE installed two shallow wells in the alluvial aquifer directly downgradient of the emplacement shaft. These two wells will be included in the EPA's annual LTHMP. The purpose of these wells is to function as early warning detection devices (for the alluvial aquifer) in the unlikely event that upward migration has occurred via the emplacement shaft. 


\subsection{Recommendations}

Based on the information provided in this report, the following tasks should be completed to fill the information gaps that remain on this project:

- Complete the human health baseline risk assessment

- Collect gas/water samples from the gas wells closest to the shot cavity

- Characterize the mudpit located by the RE-X well

- Continue the Long-Term Hydrologic Monitoring Program

- Develop an action plan in the event contamination is found

\subsection{Complete Human Health Risk Assessment}

The human health baseline risk assessment for the hydrocarbon/heavy metal contaminants is in the process of being prepared by DOE. Once it is completed it can be used to: (1) identify areas in which additional information is needed, (2) to determine the relative importance of the proposed tasks, and (3) to determine if a task is necessary.

\subsection{Collect Gas/Water Samples}

One of the potential pathways for contamination from the shot cavity to reach a receptor is by tritium migrating to one of the gas producing horizons. To check this, two wells have been identified from which gas/water samples should be collected and analyzed for tritium. Permission will have to be obtained from the owners of the wells and arrangements made to collect the samples when the owners can be present.

\subsection{Characterize the Mudpit}

During drilling of the soil borings in September 1995, drilling mud was discovered near the RE-X well. The mud was contaminated primarily with total petroleum hydrocarbons (probably diesel fuel). The Toxicity Characteristic Leaching Procedure metals analyses all came back nondetect. The vertical extent of the mud was defined but not the lateral extent. Depending on the opinion the Colorado State Department of Health takes regarding this information, additional characterization and possible cleanup may be required at this site.

\subsection{Continue the Long-Term Hydrologic Monitoring Program}

The Long-Term Hydrologic Monitoring Program should be continued and expanded to include the two monitoring wells installed onsite near SGZ during 1995. Sampling of another five wells emplaced to evaluate the impacts of the contaminated pond sediments will take place quarterly for two years. Analyses will include total petroleum hydrocarbons and metals. At the end of the 
required two-year monitoring period, if no impacts from the hydrocarbon or metals have been detected in the groundwater, the State may waive the monitoring requirement or request that these five wells be monitored on an annual basis.

\subsection{Develop an Action Plan}

Finally, a plan should be developed to specify what actions need to be taken in the event that contamination is found in any of the monitoring locations. At the present time, no plans exist that identify what happens in the event that radiological contamination is found in any of the sampling locations.

Implementing these recommendations will reduce the amount of money to be spent on the site by identifying exactly where it needs to be spent to fill data gaps and alleviate risks. It will also reduce DOE liability by allowing the investigations to focus on those areas that pose the greatest liability (if any). 


\subsection{References}

AEC (for reference, see U.S. Atomic Energy Commission).

American Indian Religious Freedom Act of 1978. Public Law 95-341.

Andrews, R. and R. Righter. 1992. Colorado Birds: A Reference to Their Distribution and Habitat. Denver, CO: Denver Museum of Natural History.

Austral, see Austral Oil Company, Inc.

Austral Oil Company, Inc. 1972. Project Rulison - Post-Shot Well Test Data: PNE-R-52. Las Vegas, NV.

Austral Oil Company, Inc. and CER Geonuclear Corporation. 1969. Project Rulison - Site Evaluation Pre-Shot R-EX Well Test Data: PNE-R-7. Las Vegas, NV.

BLM, see U.S. Bureau of Land Management.

Baumgardner, R.W., Jr. et al. 1988. "Site Selection for GRI Cooperative Tight Gas Field Research, Volume II: Geologic Characteristics of Selected Low-Permeability Gas Sandstones.” Bureau of Economic Geology Topical Report prepared for Gas Research Institute under Contract No. 5082-211-0708. Austin, TX: University of Texas.

Boysen, G.A. U.S. Environmental Protection Agency. 1976. Off-Site Radiological Safety Program For Project Rulison Flaring, Phase III: Monitoring Operations Division, EMSL-LV-539-8. Washington, DC: U.S. Government Printing Office.

Brooks, L.R., J.F. Duds, and D. Falck. U.S. Geological Survey. 1933. Land Classifications of Western Colorado. Washington, DC: U.S. Government Printing Office.

Carlson, J. U.S. Department of Agriculture. Soil Conservation Service. 1993. Personal Communication, 4 January.

CDNR, see Colorado Department of Natural Resources.

CER Corporation. 1984. Multi-Well Experiment MWX-3 As-Built Report: SAND84-7132. Las Vegas, NV.

CER Corporation. 1989. Geologic and Production Characteristics of the Tight Mesaverde Group: Piceance Basin, Colorado. Las Vegas, NV.

CER Corporation. 1992. Results of Field Verification Tests in the Tight Mesaverde Group: Piceance Basin, Colorado. Las Vegas, NV. 
CFR, see Office fo the Federal Register.

Chapman, J.B. and S.L. Hokett. U.S. Department of Energy, Nevada Operations Office. 1991. Evaluation of Groundwater Monitoring at Offsite Nuclear Test Areas. \#45085, Report DOE/NV/10845-07, UC-703. Las Vegas, NV: Desert Research Institute Water Resources Center Publication \#45085.

Claassen, H.C. 1971. Radiochemical Analyses of Water Samples From Selected Streams and Precipitation Collected October 23-November 9, 1970, in Conjunction with the First Production-Test Flaring of Gas from Test Well, Project Rulison: USGS-474-108. Denver, CO: U.S. Geological Survey.

Claassen, H.C. and P.T. Voegeli, Sr. 1971. Radiochemical Analyses of Water Samples Collected in Connection with Calibration-Test Flaring of Gas from Test Well, August 15-October 13, 1970, Project Rulison: USGS-474-107. Denver, CO: U.S. Geological Survey.

Coffin, D.L., F.A. Welder, and R.K. Glanzman. 1971. Geohydrology of the Piceance Creek Structural Basin Between the White and Colorado Rivers, Northwestern Colorado: USGS Atlas HA-370. Denver, CO: U.S. Geological Survey.

Coffin, D.L., F.A. Welder, R.K. Glanzman, and X.W. Dutton. 1968. Geohydrologic Data from the Piceance Creek Basin, between the White and Colorado Rivers, Northwestern Colorado. Colorado Groundwater Circular No. 12. Denver, CO.

Colorado. Department of Natural Resources (CDNR). Division of Wildlife. 1993. WILDATA and Wildlife Habitat Relationships. Data base accessed February 5. Grand Junction, CO.

Desert Research Institute. 1988. CERCLA Preliminary Assessment of DOE's Nevada Operations Office Nuclear Weapons Testing Areas. Las Vegas, NV.

Dickinson, W.R. and W.S. Snyder. 1978. "Plate tectonics of the Laramide orogeny." In Laramide folding associated with basement block faulting in the western United States, edited by Vincent Matthews, III. Geological Society of America Memoir 151.

DOE, see U.S. Department of Energy.

Donnell, J.R. 1968. Rulison Area Geological Maps. USGS open file.

DRI, see Desert Research Institute.

Eberline Instrument Corporation. 1977. Rulison Radiation Contamination Clearance Report: PNE-R-68. Sante Fe, NM. 
EG\&G Energy Measurements. 1995. An Aerial Radiological Survey of Project Rulison and Surrounding Area, EGG 11265-1094. Las Vegas, NV.

EL, see U.S. Army Corps of Engineers, Environmental Laboratory.

EPA, see U.S. Environmental Protection Agency.

ERDA, see U.S. Energy Research and Development Administration.

Fauver, D.N. 1986. Hazardous Waste Installation Assessment Report. Las Vegas, NV: Reynolds Electrical \& Engineering Co., Inc.

FEMA, see U.S. Federal Emergency Management Agency.

Hammerson, G.A. 1986. Amphibians and Reptiles in Colorado, Denver, CO: Colorado Division of Wildlife.

Hart, C.M., D. Engi, R.P. Fleming, and H.E. Morris. 1984. "Fracture Diagnostics Results for the Multiwell Experiment's Paludal Zone Stimulation.” Paper SPE/DOE GRI 12852 presented at the SPE/DOE Unconventional Gas Recovery Symposium, Pittsburgh, PA, 13-15 May.

Historic Properties Preservation Act of 1966. Public Law 89-665, 80 Stat. 915, 16 U.S.C.A. $\S 470$ et seq. by Public Laws 91-243, 93-54, 94-422, 94-458, 96-244, and 96-515.

Historical and Archaeological Data Preservation Act of 1974. Public Law 93-291; 88 Stat. 174; 16 U.S.C.A. § 469.

Hurr, R.T., W.W. Wilson, F.A. Welder, and R.L. Emerson. 1969. Records of Selected Wells and Springs in the Rulison Project Area, Garfield and Mesa Counties, Colorado: USGS-474-40. Denver, CO: U.S. Geological Survey.

IT, see IT Corporation.

IT Corporation. 1993a. A Class III Cultural Resources Survey of the U.S. Department of Energy Rulison Gas Stimulation Test Site, Garfield County, Colorado, DOE/NV/10972-62. Report prepared for U.S. Department of Energy, Las Vegas, NV.

IT Corporation. 1993b. Sensitive Species Survey Results for the Rulison and Rio Blanco Sites, Colorado, DOE/NV/10972-55. Report prepared for U.S. Department of Energy, Las Vegas, NV.

IT Corporation. 1993c. Floodplains and Wetlands Survey Results for the Rulison and Rio Blanco Sites, Colorado, DOE/NV/10972-59. Report prepared for U.S. Department of Energy, Las Vegas, NV. 
IT Corporation. 1995. Corrective Action Plan, Rulison Drilling Effluent Pond. Report prepared for U.S. Department of Energy, Las Vegas, NV.

Johnson, R.C., R.A. Crovelli, C.W. Spencer, and R.F. Mast. 1987. An Assessment of gas resources in Low-Permeability Sandstones of the Upper Cretaceous Mesaverde Group, Piceance Basin, Colorado. U.S. Geological Survey Open-File Report 87-357.

Knutson, C.F. 1977. Outcrop Study of Fracture Patterns and Sandstone Geometry, Eastern Uinta Basin, Utah: Study Results and Implication to Stimulation of Tight Gas 'Sands' in the Area. Report prepared for U.S. Energy Research and Development Administration, Nevada Operations Office, NVO-655-1, Las Vegas, NV.

Kollmorgen Corporation. 1975. Munsell Soil Color Charts. Baltimore, MD: Macbeth Division, Kollmorgen Corporation.

Kukal, G.C., E.H. Price, R.E. Hill, and E.R. Monson. August 1992. Results of Field Verification Tests in the Tight Mesaverde Group: Piceance Basin, Colorado, DOE Report DOE/MC/24120. Las Vegas, NV.

Larson, J.D. and W.A. Beetem. 1970. Chemical and Radiochemical Analyses of Water from Streams, Reservoirs, Wells, and Springs in the Project Rulison Area, Garfield and Mesa Counties, Colorado: USGS-474-67. Denver, CO: U.S. Geological Survey.

Lorenz, J.C. 1983. Reservoir Sedimentology of the Mesaverde Rocks at the Multi-Well Experiment Site. Sandia National Laboratories Report, SAND83-1078. Albuquerque, NM and Livermore, CA.

Lorenz, J.C. 1985. Tectonic and stress Histories of the Piceance Creek Basin and the MWX Site, from 75 Million Years Ago to the Present. Sandia National Laboratories Report, SAND84-2603. Albuquerque, NM and Livermore, CA.

Marlatt, W.E. 1973. "Climate of the Piceance Creek Basin." In An Environmental Reconnaissance of the Piceance Creek Basin, Part I, Phase I. Boulder, CO: Thorne Ecological Institute.

Mason, B.J. Battelle Memorial Institute. 1970. Letter to Ernest Campbell, 13 July.

Myal, F.R., E.H. Price, R.E. Hill, G.C. Kukal, P.J. Abadie, and C.C. Riecken. July 1989. Geologic and Production Characteristics of the Tight Mesaverde Group: Piceance Basin, Colorado. DOE Report DOE/MC/24120-2769. Las Vegas, NV.

National Environmental Policy Act of 1969, Title 42, U.S.C.S. § 4332, Section 102(2)(c). Public Law 91-190. 
Nessler, Thomas. State of Colorado, Colorado Department of Wildlife. 1995. Personal Communication, 20 April.

Nork, W.E. 1969. Radioactivity in the Hydrologic Environment Project Rulison Final Pre-Shot Report (U): U.S. Atomic Energy Commission, NVO-1229-108. Las Vegas, NV.

Nork, W.E. and P.R. Fenske. 1970. Radioactivity in Water - Project Rulison: U.S. Atomic Energy Commission, NVO-1229-131. Las Vegas, NV.

Office of the Federal Register. National Archives and Records Administration. Code of Federal Regulations, Executive Order 11593, "Protection and Enhancement of the Cultural Environment," 36 F.R. 8921. Washington, DC: GPO, May 15, 1971.

Office of the Federal Register. National Archives and Records Administration. Code of Federal Regulations, Title 10, Part 11. Washington, DC: GPO, 1974.

Office of the Federal Register. National Archives and Records Administration. Code of Federal Regulations, Title 10, "Energy," Part 1021, "National Environmental Policy Act Implementing Procedures." Washington, DC: GPO, 1992.

Office of the Federal Register. National Archives and Records Administration. Code of Federal Regulations, Title 36, "Parks, Forests, and Public Property," Part 800, "Advisory Council on Historic Preservation." Washington, DC: GPO, 1995.

Office of the Federal Register. National Archives and Records Administration. 1992. "Compliance with Floodplain Wetlands Environmental Review Requirements," Code of Federal Regulations, Title 10, Part 1022. Washington, DC: U.S. Government Printing Office.

Rand McNally. Commercial Atlas and Marketing Guide. 1993. Chicago, IL.

Reed, P.B., Jr. U.S. Fish and Wildlife Service. 1988. "National List of Plant Species that Occur in Wetlands: National Summary," Biological Report 88 (24). Washington, DC: U.S. Government Printing Office.

Reed, A.D. State Historical Society of Colorado. 1984. West Central Colorado Prehistoric Context. Denver, CO: Office of Archaeology and Historic Preservation.

Reynolds, Jr., M. 1971. Project Rulison Post-Shot Investigations Summary of Re-Entry Operations: PNE-R-45. Houston, TX: Austral Oil Company, Inc.

Reynolds, Jr., M. Austral Oil Company. 1977. The Rulison Field Massive Hydraulic Fracturing Experiment: NVO/0679-1. Report prepared for the U.S. Department of Energy, Las Vegas, NV. 
Sattler, A.R. 1984. "The Multiwell Experiment Core Program, II.": Paper SPE/DOE GRI 12854 presented at the SPE/DOE Unconventional Gas Recovery Symposium, Pittsburgh, PA: 13-15 May.

SCS, see U.S. Department of Agriculture, Soil Conservation Service.

Stosur, J.J. 1977. "Current Status of Projects Gasbuggy, Rulison, and Rio Blanco and an Appraisal of Nuclear-Explosive Fracturing Potential for the Near Future." In The Future Supply of Nature-made Petroleum and Gas; Technical Reports, edited by R.F. Meyer. New York: Pergamon Press.

Thompson, J. 1992. Fisheries Report. Grand Junction, CO: United States Bureau of Land Management.

Thompson, J. Bureau of Land Management. 1993. Personal Communication, 1 July.

Tuttle, M.L., D.J. Ackerman, W.E. Dean, P.H. Briggs, and A.J. Bartel. 1983. Chemical and Mineral Composition Data on Oil Shale and Retorted Oil-Shale Wastes From Rulison, Colorado: USGS Open File Report 83-378.

U.S. Army Corps of Engineers, Environmental Laboratory. 1987. Corps of Engineers Wetlands Delineation Manual, Technical Report Y-87-1. Vicksburg, MS.

U.S. Atomic Energy Commission. Nevada Operations Office. December 1969a. Project Rulison Post-Shot Plan and Evaluations, NVO-61. Las Vegas, NV.

U.S. Atomic Energy Commission. Nevada Operations Office. 1969b. Project Rulison Planning Directive. Las Vegas, NV.

U.S. Atomic Energy Commission. Nevada Operations Office. September 1973a. Rulison Site Cleanup Report, NVO-136. Las Vegas, NV.

U.S. Atomic Energy Commission. Nevada Operations Office. April 1973b. Project Rulison Manager's Report, NVO-71. Las Vegas, NV.

U.S. Bureau of Land Management. June 1986. State of Colorado Wilderness Map. Denver, $\mathrm{CO}$.

U.S. Bureau of Land Management. 1980. Recreation Management Areas--Bureau of Land Management-Colorado. Revised map. Denver, CO.

U.S. Congress. Office of Technology Assessment. October 1989. The Containment of Underground Nuclear Explosions. OTA-ISC-414. Washington, DC: U.S. Government Printing Office. 
U.S. Department of Agriculture. Soil Conservation Service. 1980. Soil Survey of Rifle Area, Colorado. Washington, DC: U.S. Government Printing Office.

U.S. Department of Agriculture. Soil Conservation Service. 1983. National Soils Handbook. Washington, DC: U.S. Government Printing Office.

U.S. Department of Agriculture. Soil Conservation Service. 1987. Hydric Soils of the United States. In cooperation with the National Technical Committee for Hydric Soils. Washington, DC: U.S. Government Printing Office.

U.S. Department of Energy. Nevada Operations Office. 1984. Long-Term Hydrologic Monitoring Program Rulison Event Site, Grand Valley, Colorado, NVO-273. Las Vegas, NV.

U.S. Energy Research and Development Administration. 1976. Project Rulison Well Plugging and Site Abandonment Plan, NVO-174. Las Vegas, NV.

U.S. Energy Research and Development Administration. 1977. Project Rulison Well Plugging and Site Abandonment Final Report, NVO-187. Las Vegas, NV.

U.S. Environmental Protection Agency. 1974. Off-Site Surveillance Activities of the National Environmental Research Center from July through December 1970, NERC-LV-539-17. Las Vegas, NV.

U.S. Geological Survey. 1970. Geohydrology--Project Rulison, Garfield County, Colorado, With a Section on Aquifer Response, USGS-474-68. Denver, CO.

U.S. Geological Survey. 1980. Rulison, Colorado, and 1952 Rock School, Colorado, 7.5 Minute Quadrangle Maps. Denver, CO.

U.S. Geological Survey. 1987. National Wilderness Preservation System, map. Denver, CO.

U.S. Federal Emergency Management Agency. January 3, 1986. Flood Insurance Rate Map, Garfield County, Colorado (Unincorporated areas), Map Index, Community-Panel Numbers 080205 0001-1900. Washington, DC: U.S. Government Printing Office.

USDA, see U.S. Department of Agriculture.

USGS, see U.S. Geological Survey.

Voegeli, P.T., Sr. 1969. Geology and Hydrology of the Project Rulison Exploratory Hole, Garfield County, Colorado, USGS-474-16. Denver, CO: U.S. Geological Survey.

Voegeli, P.T., Sr., S.W. West, and E.H. Cordes. 1970. Geohydrology - Project Rulison, Garfield County, Colorado, USGS-474-68. Denver, CO: U.S. Geological Survey. 
Voegeli, P.T., Sr., and H.C. Claassen. 1971a. Radiochemical Analyses of Water from Selected Streams, Wells, Springs, and Precipitation Collected Prior to Reentry Drilling, Project Rulison, USGS-474-83. Denver, CO: U.S. Geological Survey.

Voegeli, P.T., Sr., and H.C. Claassen. 1971b. Radiochemical Analyses of Water from Selected Streams, Wells, Springs, and Precipitation Collected During Reentry Drilling, Project Rulison, USGS-474-101. Denver, CO: U.S. Geological Survey.

Whitney, S. 1992. Western Forests, Audobon Society Nature Guides. New York: Alfred A. Knoff, Inc.

Yeend, W.E. 1968. Quaternary Geology of the Grand-Battlement Mesa Area, Colorado. USGS Open File Report. 


\section{Appendix A \\ Project Rulison, Long-Term \\ Hydrologic Monitoring Program \\ Analytical Results, 1972-1994, Garfield County, Colorado}


Rulison Tritiun Results in pCl/L for 1972-1982

\begin{tabular}{|c|c|c|c|c|c|c|c|c|c|c|c|c|c|c|c|c|c|c|c|c|c|c|}
\hline & \multicolumn{2}{|c|}{1972} & \multicolumn{2}{|c|}{1973} & \multicolumn{2}{|c|}{1974} & \multicolumn{2}{|c|}{1975} & \multicolumn{2}{|c|}{1976} & \multicolumn{2}{|c|}{1977} & \multicolumn{2}{|c|}{1978} & \multicolumn{2}{|c|}{1979} & \multicolumn{2}{|c|}{1980} & \multicolumn{2}{|c|}{1981} & \multicolumn{2}{|c|}{1982} \\
\hline & $3 \mathrm{H}$ & $3 H^{+}$ & $3 \mathrm{H}$ & $3 H^{+}$ & $3 H$ & $3 H^{+}$ & $3 \mathrm{H}$ & $3 H^{+}$ & $3 \mathrm{H}$ & $3 \mathrm{H}^{+}$ & $3 \mathrm{H}$ & $3 \mathrm{H}^{+}$ & $3 \mathrm{H}$ & $3 \mathrm{H}^{+}$ & $3 \mathrm{H}$ & $3 H^{*}$ & $3 \mathrm{H}$ & $3 \mathrm{H}^{+}$ & $3 \mathrm{H}$ & $3 \mathrm{H}^{+}$ & $3 \mathrm{H}$ & $3 \mathrm{H}^{+}$ \\
\hline HAYHARD RANCH WELL & $\begin{array}{l}380 \pm \\
230^{-}\end{array}$ & & $=300$ & & $\stackrel{480 \pm}{13^{-}}$ & & $\begin{array}{c}350 \pm \\
12\end{array}$ & & $\begin{array}{l}480 \pm \\
210^{-}\end{array}$ & & $\begin{array}{l}440 \pm \\
230^{-}\end{array}$ & & $\begin{array}{l}710 \pm \\
350\end{array}$ & & & $390 \pm$ & & $\begin{array}{c}330 \pm \\
11\end{array}$ & & $\begin{array}{c}360 \pm \\
10^{-}\end{array}$ & & $\begin{array}{l}370 \pm \\
8.4^{-}\end{array}$ \\
\hline $\begin{array}{l}\text { SEARCY RANCH WELL } \\
\text { (SCHWAB) }\end{array}$ & $\begin{array}{l}740 \pm \\
240^{-}\end{array}$ & & $\begin{array}{l}670 \pm \\
250^{-}\end{array}$ & & $\underset{16}{800 \pm}$ & & $\frac{380 \pm}{13}$ & & $\begin{array}{l}740+ \\
270^{-}\end{array}$ & & & ${ }_{14}^{430 \pm}$ & $\begin{array}{l}690 \pm \\
350\end{array}$ & & & $\begin{array}{c}440 \pm \\
11\end{array}$ & & ${ }_{11}^{360 \pm}$ & & $\begin{array}{l}250 \pm \\
8.9^{-}\end{array}$ & & $\begin{array}{l}320 \pm \\
8.3^{-}\end{array}$ \\
\hline GARDNER RANCH WELL & $\begin{array}{l}770 \pm \\
240\end{array}$ & & $\begin{array}{l}420 \pm \\
240^{-}\end{array}$ & & $\begin{array}{c}510 \pm \\
12 .\end{array}$ & & $\underset{15}{510 t}$ & & $\begin{array}{l}610^{-} \\
270^{-}\end{array}$ & $\begin{array}{l}310^{ \pm} \\
9.8^{-}\end{array}$ & $\begin{array}{l}390 \pm \\
230\end{array}$ & & $\begin{array}{l}650 \pm \\
350^{-}\end{array}$ & & & $\begin{array}{l}310^{-} \\
9.8^{-}\end{array}$ & & $\frac{300 \pm}{10^{ \pm}}$ & & $\begin{array}{l}240 \pm \\
8.8^{-}\end{array}$ & & $\begin{array}{l}250 \pm \\
7.7\end{array}$ \\
\hline SEFCOVIC RANCH WELL & & & & & & & $\frac{580 \pm}{15}$ & & ${ }_{11}^{420 \pm}$ & & $\begin{array}{l}520 \pm \\
240^{-}\end{array}$ & & $\begin{array}{l}880 \pm \\
350\end{array}$ & & & $\begin{array}{c}300 \pm \\
10\end{array}$ & & $110 \pm$ & & $\begin{array}{l}290+ \\
9.5\end{array}$ & & $\begin{array}{l}320 \pm \\
8.4^{-}\end{array}$ \\
\hline CER IEST WELL & $\begin{array}{l}770 \pm \\
240\end{array}$ & & $\begin{array}{l}800 \pm \\
250^{-}\end{array}$ & & $\underset{15}{610 \pm}$ & & $\begin{array}{c}540 \pm \\
16\end{array}$ & & ${ }_{11}^{350 \pm}$ & & $\begin{array}{l}560 \pm \\
240^{+}\end{array}$ & & $\begin{array}{l}580 \pm \\
350^{-}\end{array}$ & & & $\begin{array}{l}230 \pm \\
8.9^{-}\end{array}$ & & $\begin{array}{l}240 \pm \\
9.6\end{array}$ & & $\begin{array}{l}190 \pm \\
7.7\end{array}$ & & $\begin{array}{l}280 \pm \\
8.0\end{array}$ \\
\hline BERNKLAU RANCH HELL & $\begin{array}{l}250 \pm \\
230\end{array}$ & & $\begin{array}{l}320 \pm \\
240^{-}\end{array}$ & & $350 \pm$ & & $\underset{19}{510 \pm}$ & & $\begin{array}{l}350+ \\
9.6\end{array}$ & & & & & & & & & & & & & \\
\hline $\begin{array}{l}\text { GRAND YALLEY CITY } \\
\text { SUPPLY (SPRING) }\end{array}$ & $\begin{array}{l}270 \pm \\
230\end{array}$ & & $<300$ & & ${ }_{11}^{170 \pm}$ & & $\begin{array}{l}130 \pm \\
9.6\end{array}$ & & -6 & & & $\begin{array}{l}564 \\
8.3\end{array}$ & & $<20$ & & $\begin{array}{r}40 \pm \\
6.8^{ \pm}\end{array}$ & & $\underset{6.6}{31 \pm}$ & & $6.1^{16 \pm}$ & & $\begin{array}{c}74 \pm \\
5.8\end{array}$ \\
\hline POTIER RANCH SPRING & & & & & & & & & & & $\begin{array}{l}460 \pm \\
240^{-}\end{array}$ & & $\begin{array}{l}680 \pm \\
350^{-}\end{array}$ & & & $\begin{array}{l}280 \pm \\
9.5\end{array}$ & & $\begin{array}{l}230 \pm \\
9.4^{-}\end{array}$ & & $\begin{array}{l}210 \pm \\
8.2\end{array}$ & & $\begin{array}{l}270 \pm \\
7.6\end{array}$ \\
\hline $\begin{array}{l}\text { SPRING ( } 300 \text { YDS. } \\
\text { M.W. OF GZ) }\end{array}$ & $\begin{array}{l}510 \pm \\
230^{-}\end{array}$ & & $\begin{array}{l}740 \pm \\
250^{-}\end{array}$ & & $\begin{array}{c}450 \pm \\
13\end{array}$ & & $\underset{16}{480 \pm}$ & & $\begin{array}{l}270 \pm \\
9.3\end{array}$ & & & ${ }_{11}^{170+}$ & $\begin{array}{l}730 \pm \\
350\end{array}$ & & & $\begin{array}{l}180 \pm \\
8.5\end{array}$ & & $\begin{array}{l}210 \pm \\
9,2\end{array}$ & & $130 \pm$ & & $\begin{array}{l}190 \pm \\
7.1^{-}\end{array}$ \\
\hline $\begin{array}{l}\text { BAITLEMENT CREEK } \\
\text { (SURFACE) }\end{array}$ & $\begin{array}{l}860^{ \pm} \\
240^{\circ}\end{array}$ & & $\begin{array}{l}510 \pm \\
240^{-}\end{array}$ & & $\underset{15}{580 \pm}$ & & ${ }_{12}^{300 \pm}$ & & $250 \pm$ & & & $\begin{array}{l}330 \pm \\
13\end{array}$ & $\begin{array}{l}850+ \\
350^{-}\end{array}$ & & & $\begin{array}{l}240 \pm \\
9.1^{-}\end{array}$ & & $\begin{array}{l}1,0 \pm \\
8,2\end{array}$ & & $\begin{array}{l}200 \pm \\
8.5\end{array}$ & & $190 \pm$ \\
\hline
\end{tabular}

(U.S. DOE, 1984) 


\section{Project Rulison, Long-Term Hydrological Monitoring Program Analytical Results, 1983-1985}

\section{Grand Valley, Colorado}

City Spring

Albent Gardner Ranch Well

Battlement Creek (surface)

Spring 300 yards NW of GZ

\section{Rulison, Colorado}

Lee Hayward Ranch Well

Potter Ranch Well

Robert Searcy (G. Schwab) Ranch Well

Felix Sefcovic Ranch Well

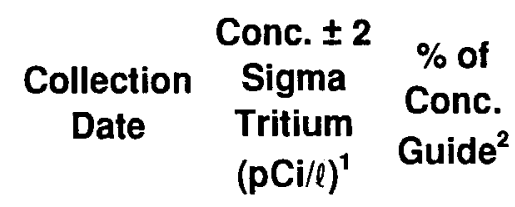

\section{$5 / 29 / 83$}

$5 / 29 / 83$

$5 / 30 / 83$

$5 / 30 / 83$

$5 / 30 / 83$

$\begin{array}{cc}110 \pm 6 & 0.6 \\ 260 \pm 7 & 1 \\ 200 \pm 7 & 1 \\ \cdots & --- \\ \cdots-- & -\cdots\end{array}$

\section{$5 / 29 / 83$}

$5 / 30 / 83$

$5 / 29 / 83$

$5 / 29 / 83$

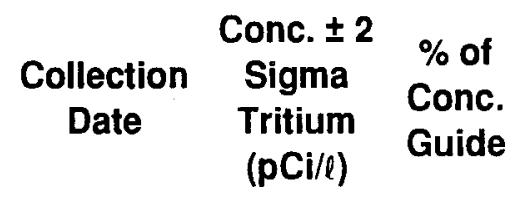

$6 / 20 / 84$

$6 / 21 / 84$

$6 / 20 / 84$

$6 / 20 / 84$

$6 / 20 / 84$

$\begin{array}{cc}3.3 \pm 5.0^{\star} & <0.02 \\ 200 \pm 6 & 1 \\ 120 \pm 5 & 0.6 \\ 130 \pm 6 & 0.6 \\ 110 \pm 6 & 0.6\end{array}$

$6 / 21 / 84$

$6 / 21 / 84$

$6 / 21 / 84$

$6 / 21 / 84$

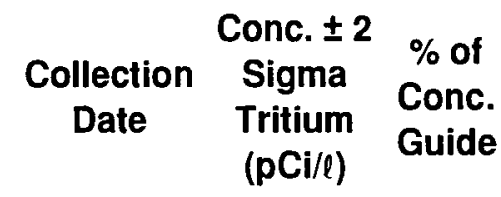

$6 / 20 / 85$

$6 / 19 / 85$

$6 / 19 / 85$

$6 / 19 / 85$

$6 / 19 / 85$

$\begin{array}{cc}-6.2 \pm 7.7^{\star} & <0.01 \\ 200 \pm 8 & 1 \\ 130 \pm 8 & 0.6 \\ 130 \pm 8 & 0.6 \\ 210 \pm 9 & 1\end{array}$

$6 / 20 / 85$

$6 / 20 / 85$

$6 / 20 / 85$

$6 / 20 / 85$
$280 \pm 9 \quad 1$

$150 \pm 8 \quad 0.8$

$170 \pm 9 \quad 0.9$

$210 \pm 8 \quad 1$

\section{'Picocurie per liter (pCi//)}

${ }^{2}$ Established by DOE Order as $90,000 \mathrm{pCi} / \ell$ tritium.

${ }^{*}=$ Concentration is less than the minimum detectable concentration (MDC).

$\mathrm{NA}=$ Not applicable. Percent of concentration guide is not applicable either because the tritium result is less than the MDC or because the water is known to be nonpotable. 


\section{Project Rulison, Long-Term Hydrological Monitoring Program Analytical Results, 1986-1988}

\begin{tabular}{|c|c|c|c|c|c|c|c|c|c|}
\hline & $\begin{array}{l}\text { Collection } \\
\text { Date }\end{array}$ & $\begin{array}{c}\text { Conc. } \pm 2 \\
\text { Sigma } \\
\text { Tritium } \\
(\mathrm{pCi} / \mathrm{l})^{1}\end{array}$ & $\begin{array}{c}\% \text { of } \\
\text { Conc. } \\
\text { Guide }^{2}\end{array}$ & $\begin{array}{l}\text { Collection } \\
\text { Date }\end{array}$ & $\begin{array}{c}\text { Conc. } \pm 2 \\
\text { Sigma } \\
\text { Tritium } \\
(\mathrm{pCi} / \mathrm{l})\end{array}$ & $\begin{array}{l}\% \text { of } \\
\text { Conc. } \\
\text { Guide }\end{array}$ & $\begin{array}{l}\text { Collection } \\
\text { Date }\end{array}$ & $\begin{array}{c}\text { Conc. } \pm 2 \\
\text { Sigma } \\
\text { Tritium } \\
(p \mathrm{ci} / \ell)\end{array}$ & $\begin{array}{l}\% \text { of } \\
\text { Conc. } \\
\text { Guide }\end{array}$ \\
\hline \multicolumn{10}{|l|}{ Grand Valley, Colorado } \\
\hline City Spring & $6 / 15 / 86$ & $28 \pm 8$ & 0.1 & $7 / 27 / 87$ & $-0.13 \pm 8.2^{*}$ & $<0.01$ & $6 / 25 / 88$ & $-2 \pm 16^{\star}$ & $<0.01$ \\
\hline Albert Gardner Ranch Well & $6 / 15 / 86$ & $190 \pm 9$ & 1 & $7 / 27 / 87$ & $170 \pm 8$ & 0.8 & $6 / 25 / 88$ & $170 \pm 12$ & 0.86 \\
\hline Battlement Creek (surface) & $6 / 15 / 86$ & $100 \pm 8$ & 0.5 & $7 / 27 / 87$ & $100 \pm 8$ & 0.5 & $6 / 25 / 88$ & $140 \pm 11$ & 0.70 \\
\hline Spring 300 yards NW of GZ & -...- & ..... & $\ldots$ & $7 / 27 / 87$ & $87 \pm 8$ & 0.4 & $6 / 25 / 88$ & $84 \pm 11$ & 0.42 \\
\hline CER Test Well & $6 / 15 / 86$ & N/A & $\cdots$ & $7 / 27 / 87$ & $160 \pm 9$ & 0.8 & $6 / 25 / 88$ & $160 \pm 12$ & 0.79 \\
\hline \multicolumn{10}{|l|}{ Rulison, Colorado } \\
\hline Lee Hayward Ranch Well & $6 / 15 / 86$ & $260 \pm 12$ & 1 & $7 / 27 / 87$ & $220 \pm 8$ & 1 & $6 / 25 / 88$ & $250 \pm 12$ & 1.24 \\
\hline Potter Ranch Well & $6 / 16 / 86$ & $140+8$ & 0.7 & $7 / 27 / 87$ & $120 \pm 8$ & 06 & $6 / 27 / 88$ & $140 \pm 11$ & 0.71 \\
\hline Robert Searcy (G. Schwab) Ranch Well & $6 / 15 / 86$ & $90 \pm 9$ & 0.5 & $7 / 27 / 87$ & $160 \pm 9$ & 0.8 & $6 / 25 / 88$ & $150 \pm 11$ & 0.76 \\
\hline Felix Sefcovic Ranch Well & $\begin{array}{l}6 / 15 / 86 \\
6 / 15 / 86\end{array}$ & $\begin{array}{c}98 \pm 8 \\
190 \pm 8\end{array}$ & 0.5 & $7 / 27 / 87$ & $170 \pm 8$ & 0.8 & $6 / 25 / 88$ & $160 \pm 11$ & 0.82 \\
\hline
\end{tabular}

${ }^{1}$ Picocurie per liter ( $\left.\mathrm{pCi} / \ell\right)$

${ }^{2}$ Established by DOE Order as $90,000 \mathrm{pCi} / \ell$ tritium.

* $=$ Concentration is less than the minimum detectable concentration (MDC).

NA $=$ Not applicable. Percent of concentration guide is not applicable either because the tritium result is less than the MDC or because the water is known to be nonpotable. 


\section{Project Rulison, Long-Term Hydrological Monitoring Program Analytical Results, 1989-1991}

\begin{tabular}{|c|c|c|c|c|c|c|c|c|c|}
\hline & $\begin{array}{l}\text { Collection } \\
\text { Date }\end{array}$ & $\begin{array}{c}\text { Conc. } \pm 2 \\
\text { Sigma } \\
\text { Tritium } \\
(p C i / l)^{1}\end{array}$ & $\begin{array}{l}\% \text { of } \\
\text { Conc. } \\
\text { Guide }^{2}\end{array}$ & $\begin{array}{l}\text { Collection } \\
\text { Date }\end{array}$ & $\begin{array}{c}\text { Conc. } \pm 1 \\
\text { Sigma } \\
\text { Tritium } \\
(\mathrm{pCi} / \mathrm{l})\end{array}$ & $\begin{array}{l}\% \text { of } \\
\text { Conc. } \\
\text { Guide }\end{array}$ & $\begin{array}{l}\text { Collection } \\
\text { Date }\end{array}$ & $\begin{array}{c}\text { Conc. } \pm 1 \\
\text { Sigma } \\
\text { Tritium } \\
\text { (pCi/l) }\end{array}$ & $\begin{array}{l}\% \text { of } \\
\text { Conc. } \\
\text { Guide }\end{array}$ \\
\hline \multicolumn{10}{|l|}{ Grand Valley, Colorado } \\
\hline City Spring & $6 / 13 / 89$ & $1.1 \pm 6.8^{*}$ & 0.01 & $6 / 19 / 90$ & $9.9 \pm 4.1^{\star}$ & 0.05 & $6 / 11 / 91$ & $0.78 \pm 3.12^{*}$ & NA \\
\hline Albert Gardner Ranch Well & $6 / 13 / 89$ & $140 \pm 8$ & 0.70 & $6 / 19 / 90$ & $87 \pm 5.0$ & 0.43 & $6 / 11 / 91$ & $113 \pm 4$ & 0.6 \\
\hline Battlement Creek (surface) & $6 / 13 / 89$ & $86 \pm 8$ & 0.43 & $6 / 19 / 90$ & $22 \pm 2.2$ & 0.11 & $6 / 11 / 91$ & $56 \pm 3$ & 0.3 \\
\hline Spring 300 yards NW of GZ & $6 / 13 / 89$ & $73 \pm 7$ & 0.36 & $6 / 19 / 90$ & $18 \pm 2.0$ & 0.09 & $6 / 11 / 91$ & $57 \pm 3$ & 0.3 \\
\hline CER Test Well & $6 / 13 / 89$ & $140 \pm 8$ & 0.70 & $6 / 19 / 90$ & $41 \pm 2.2$ & 0.21 & $6 / 11 / 91$ & $57 \pm 2.1$ & 0.3 \\
\hline \multicolumn{10}{|l|}{ Rulison, Colorado } \\
\hline Lee Hayward Ranch Well & $6 / 13 / 89$ & $170 \pm 8$ & 0.85 & $6 / 19 / 90$ & $88 \pm 2.7$ & 0.44 & $6 / 11 / 91$ & $187 \pm 4$ & 0.9 \\
\hline Potter Ranch Well & $6 / 13 / 89$ & $120 \pm 8$ & 0.6 & $6 / 19 / 90$ & $43 \pm 2.1$ & 0.22 & $6 / 11 / 91$ & $119 \pm 4$ & 0.6 \\
\hline Robert Searcy (G. Schwab) Ranch Well & $6 / 13 / 89$ & $89 \pm 8$ & 0.45 & $6 / 19 / 90$ & $41 \pm 2.8$ & 0.21 & $6 / 11 / 91$ & $63 \pm 4$ & 0.3 \\
\hline Felix Sefcovic Ranch Well & $6 / 13 / 89$ & $77 \pm 8$ & 0.38 & $6 / 19 / 90$ & $27 \pm 2.6$ & 0.13 & $6 / 11 / 91$ & $133 \pm 4$ & 0.7 \\
\hline
\end{tabular}

${ }^{1}$ Picocurie per liter (pCi/l)

${ }^{2}$ Established by DOE Order as $90,000 \mathrm{pCi} / /$ tritium.

* $=$ Concentration is less than the minimum detectable concentration (MDC).

$\mathrm{NA}=$ Not applicable. Percent of concentration guide is not applicable either because the tritium result is less than the MDC or because the water is known to be nonpotable. 


\section{Project Rulison, Long-Term Hydrological Monitoring Program Analytical Results, 1992-1994}

\begin{tabular}{|c|c|c|c|c|c|c|c|c|c|}
\hline . & $\begin{array}{l}\text { Collection } \\
\text { Date }\end{array}$ & $\begin{array}{c}\text { Conc. } \pm 2 \\
\text { Sigma } \\
\text { Tritium } \\
(\mathrm{pCi} / \mathrm{l})^{1}\end{array}$ & $\begin{array}{l}\% \text { of } \\
\text { Conc. } \\
\text { Guide }^{2}\end{array}$ & $\begin{array}{l}\text { Collection } \\
\text { Date }\end{array}$ & $\begin{array}{c}\text { Conc. } \pm 2 \\
\text { Sigma Tritium } \\
(p \mathrm{Ci} / \ell)\end{array}$ & $\begin{array}{l}\% \text { of } \\
\text { Conc. } \\
\text { Guide }\end{array}$ & $\begin{array}{c}\text { Collection } \\
\text { Date }\end{array}$ & $\begin{array}{c}\text { Conc. } \pm 1 \\
\text { Sigma Tritium } \\
(\mathrm{pCi} / /)\end{array}$ & $\begin{array}{l}\% \text { of } \\
\text { Conc. } \\
\text { Guide }\end{array}$ \\
\hline \multicolumn{10}{|l|}{ Grand Valley, Colorado } \\
\hline City Spring & $6 / 9 / 92$ & $0.43 \pm 1.49^{*}$ & NA & $6 / 16 / 93$ & $-1.6 \pm 3.1^{*}$ & NA & $5 / 29 / 94$ & $-1.2 \pm 3.5^{\star}$ & NA \\
\hline Albert Gardner Ranch Well & $6 / 9 / 92$ & $98 \pm 3$ & 0.11 & $6 / 16 / 93$ & $80 \pm 4.4$ & 0.09 & $5 / 29 / 94$ & $82 \pm 5.1$ & 0.09 \\
\hline Battlement Creek (surface) & $6 / 9 / 92$ & $63 \pm 2$ & 0.07 & $6 / 16 / 93$ & $49 \pm 3.8$ & 0.05 & $5 / 29 / 94$ & $48 \pm 4.2$ & 0.05 \\
\hline Spring 300 yards NW of GZ & 6/9/92 & $63 \pm 2$ & 0.07 & $6 / 16 / 93$ & $57 \pm 4.2$ & 0.06 & $5 / 29 / 94$ & $47 \pm 4.0$ & 0.05 \\
\hline CER Test Well & $6 / 9 / 92$ & $48 \pm 2$ & 50.05 & $6 / 16 / 93$ & $51 \pm 4.2$ & 0.06 & $5 / 29 / 94$ & $84 \pm 4.6$ & 0.09 \\
\hline \multicolumn{10}{|l|}{ Rulison, Colorado } \\
\hline Lee Hayward Ranch Well & 6/9/92 & $160 \pm 3$ & 0.18 & $6 / 16 / 93$ & $116 \pm 5.2^{\star}$ & 0.02 & $5 / 29 / 94$ & $100 \pm 4.6^{\star}$ & 0.11 \\
\hline Potter Ranch Well & $6 / 9 / 92$ & $67 \pm 2$ & 0.07 & $6 / 16 / 93$ & $1.4 \pm 2.9$ & 0.002 & $5 / 29 / 94$ & $82 \pm 4.6$ & 0.09 \\
\hline Rothgery (Searcy) (Schwab) Ranch Well & $6 / 9 / 92$ & $78 \pm 2$ & 0.09 & $6 / 16 / 93$ & $57 \pm 4.1$ & 0.06 & $5 / 29 / 94$ & $71 \pm 4.7$ & 0.08 \\
\hline Felix Sefcovic Ranch Well & 6/9/92 & $57 \pm 2$ & 0.06 & $6 / 16 / 93$ & $100 \pm 4.9^{*}$ & 0.11 & $5 / 29 / 94$ & $87 \pm 4.4$ & 0.10 \\
\hline
\end{tabular}

${ }^{1}$ Picocurie per liter (pCi/l)

${ }^{2}$ Established by DOE Order as $90,000 \mathrm{pCi} / \ell$ tritium.

${ }^{*}=$ Concentration is less than the minimum detectable concentration (MDC).

$N A=$ Not applicable. Percent of concentration guide is not applicable either because

the tritium result is less than the MDC or because the water is known to be nonpotable. 


\section{Appendix B \\ Records of Selected Wells and Springs, Rulison Project Area}


Records of selected wells, Rultson project area, Garfield and Mesa Count ies, culurado

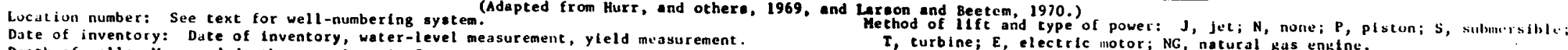

(accuracy $0.5 \mathrm{ft}$ ); reported depths ore

Altitude of land surface: Altitude, estimated from $7 \frac{1}{2}$-minute yuadiangle topographic maps, is given in leet above mean sea level.

Deth tenth below land surface; reported depths are given in feet below land surface. A "P"

Yield: All quantities are given in gallons per minute. R, repurted;

Use of water:

5 , stock.

number.

Remarks: DC, depeh well cased; $P E$, perfurated casing with interval shown

\begin{tabular}{|c|c|c|c|c|c|c|c|c|c|c|c|c|c|c|c|c|}
\hline \multirow{2}{*}{\multicolumn{2}{|c|}{$\begin{array}{l}\text { Lucation } \\
\text { number }\end{array}$}} & \multirow{2}{*}{$\begin{array}{c}\text { Ormer } \\
\text { or } \\
\text { tenant }\end{array}$} & \multirow{2}{*}{$\begin{array}{c}\text { Dace } \\
\text { of } \\
\text { Inventory }\end{array}$} & \multirow{2}{*}{$\begin{array}{c}\text { Year } \\
\text { com- } \\
\text { pleted }\end{array}$} & \multirow{2}{*}{$\begin{array}{c}\text { Depth } \\
\text { of } \\
\text { well } \\
\text { (feet) }\end{array}$} & \multicolumn{2}{|c|}{ Casing } & \multirow{2}{*}{$\begin{array}{c}\text { Altitude } \\
\text { of } \\
\text { lond } \\
\text { surface } \\
\text { (feet) }\end{array}$} & \multirow{2}{*}{$\begin{array}{l}\text { Depth } \\
\text { to } \\
\text { woter } \\
\text { (feet) }\end{array}$} & \multirow{2}{*}{$\begin{array}{c}\text { Method } \\
\text { of } \\
11 \mathrm{ft} \\
\text { and } \\
\text { power } \\
\end{array}$} & \multirow{2}{*}{$\begin{array}{c}\text { yield } \\
\text { (gallons } \\
\text { per } \\
\text { minute) }\end{array}$} & \multirow[b]{2}{*}{$\begin{array}{c}\text { Use } \\
\text { of } \\
\text { water }\end{array}$} & \multirow{2}{*}{$\begin{array}{c}\text { ronper - } \\
\text { ature } \\
\text { of water } \\
\left({ }^{\circ} \mathrm{c}\right)\end{array}$} & \multirow{2}{*}{$\begin{array}{c}\text { Turbid- } \\
\text { ity } \\
\text { (mllll- } \\
\text { grams } \\
\text { per liter) }\end{array}$} & \multirow[b]{2}{*}{$\begin{array}{c}\text { Well } \\
\text { permit } \\
\text { number }\end{array}$} & \multirow[b]{2}{*}{ K.m.nk:i } \\
\hline & & & & & & \begin{tabular}{l|} 
Dlameter \\
(Inchea)
\end{tabular} & Type & & & & & & & & & \\
\hline \multirow[t]{3}{*}{$\begin{array}{l}\text { SC } \\
\text { SC }\end{array}$} & $\begin{array}{r}5-92-33 \text { aac } \\
6-93-15 c b d \\
-16 b c b\end{array}$ & $\begin{array}{l}\text { W. Jewell } \\
\text { K. Johnson } \\
\text { Kozy Kot tage } \\
\text { Kourt }\end{array}$ & $\begin{array}{r}10-22-69 \\
3-26-69 \\
3-27-69\end{array}$ & $\begin{array}{l}1962 \\
1941 \\
1954\end{array}$ & $\begin{array}{l}35 \\
41 \\
50\end{array}$ & $\begin{array}{r}6 \\
-6\end{array}$ & $\begin{array}{c}\text { Steel } \\
-- \\
--\end{array}$ & $\begin{array}{l}5,690 \\
5,330 \\
5,300\end{array}$ & $\begin{array}{l}6.5 \\
25 \\
20\end{array}$ & $\begin{array}{r}\mathbf{J}, \mathbf{E} \\
\mathbf{J , E} \\
--\end{array}$ & $\begin{array}{l}-- \\
- \\
60 R\end{array}$ & $\begin{array}{l}D, S \\
D \\
D\end{array}$ & $\ddot{10}$ & $\begin{array}{l}- \\
1 \\
--\end{array}$ & $\begin{array}{c}\text { P12707 } \\
\quad-- \\
\text { R11 }\end{array}$ & \multirow{3}{*}{ 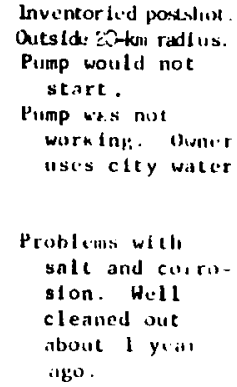 } \\
\hline & $-16 c d b$ & J. Layne & $3-27-69$ & 1963 & 40 & 7 & Steel & 5,510 & 19 & J,E & $20 R$ & $D, S$ & $\cdots$ & -. & +18318 & \\
\hline & $\begin{array}{l}-16 c d d \\
-16 d c c \\
-17 b b d\end{array}$ & $\begin{array}{l}\text { W. Wood } \\
\text { R. Swallow } \\
\text { w. Shafto }\end{array}$ & $\begin{array}{l}1-27-69 \\
3-27-69 \\
3-26-69\end{array}$ & $\begin{array}{l}1964 \\
1964 \\
1956\end{array}$ & $\begin{array}{l}24 \\
44 \\
38\end{array}$ & $\begin{array}{l}7 \\
7 \\
5\end{array}$ & $\begin{array}{l}\text { Steel } \\
\text { Steel } \\
\text { Steel }\end{array}$ & $\begin{array}{l}5,305 \\
5,315 \\
5,290\end{array}$ & $\begin{array}{r}8 \\
18 \\
18\end{array}$ & $\begin{array}{l}J, E \\
, E\end{array}$ & $\begin{array}{c}20 R \\
20 R \\
5 E\end{array}$ & $\begin{array}{l}N \\
D \\
D\end{array}$ & $\bar{u}$ & $\begin{array}{l}-- \\
-i\end{array}$ & 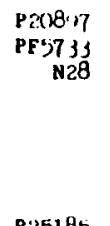 & \\
\hline
\end{tabular}

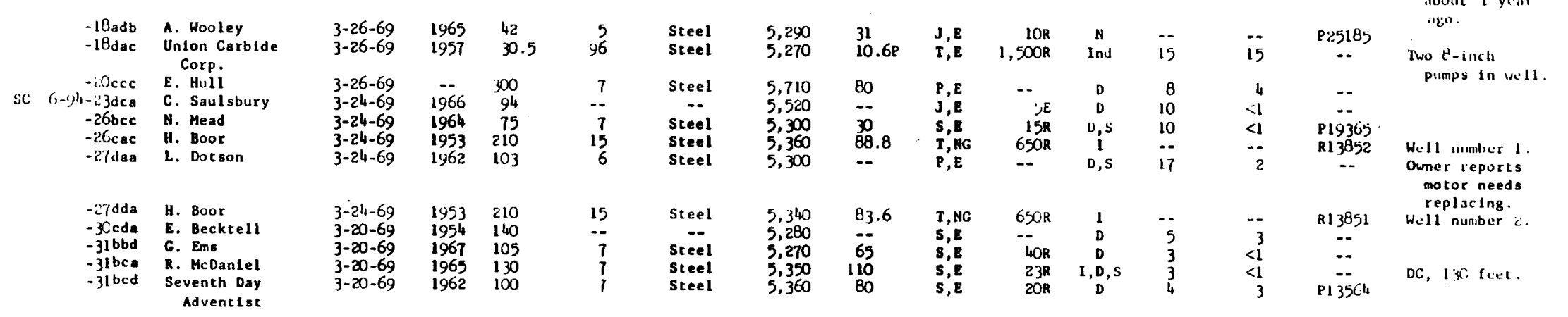


- - Records of selected vells, Rullson project area, Garfleld and Hese Countles, Colorado--Cont Inued

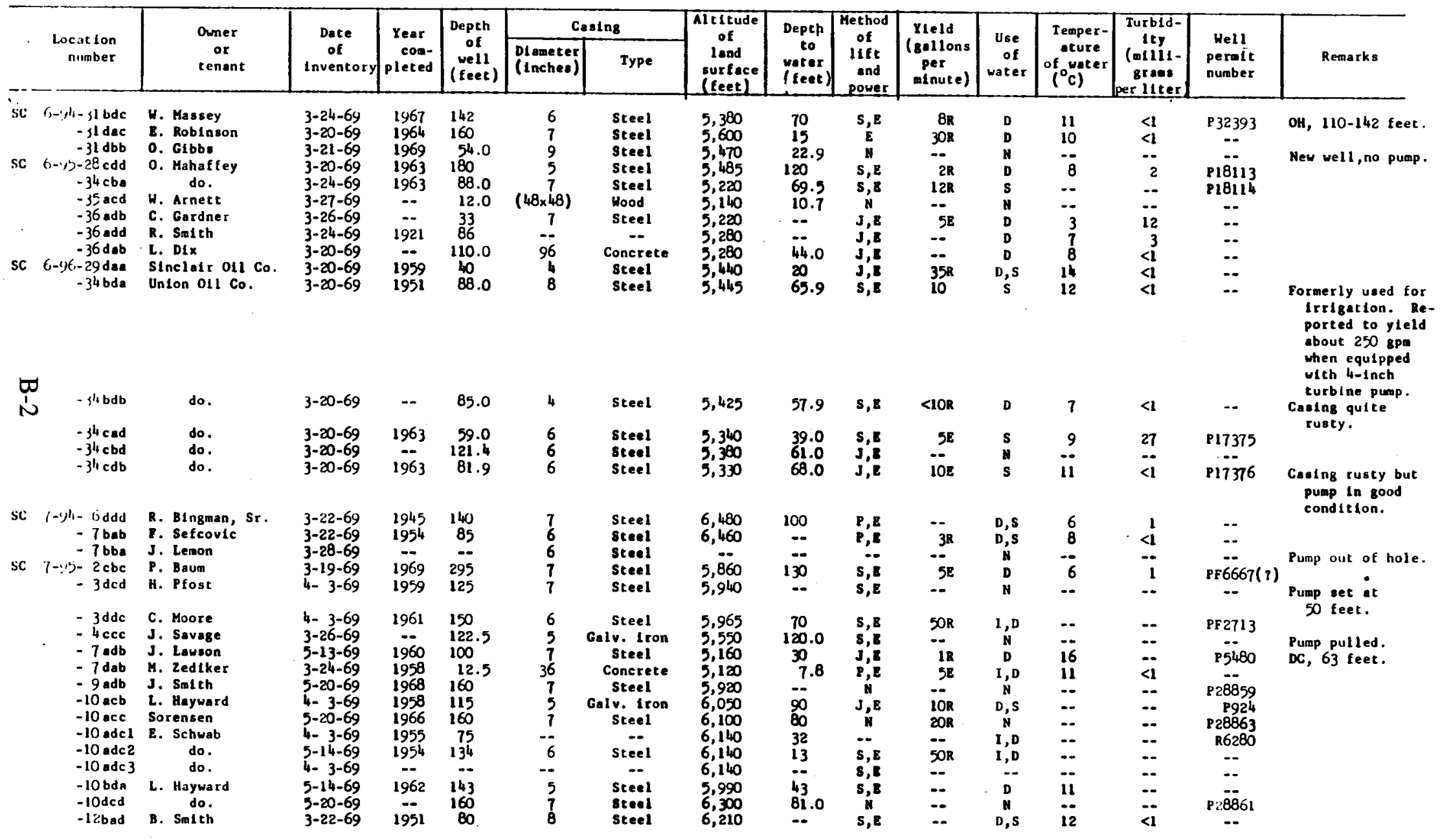


- -Records of selected uells, Rullson project eree, Garfleld and Mesa Count les, Colorado--Cont inued

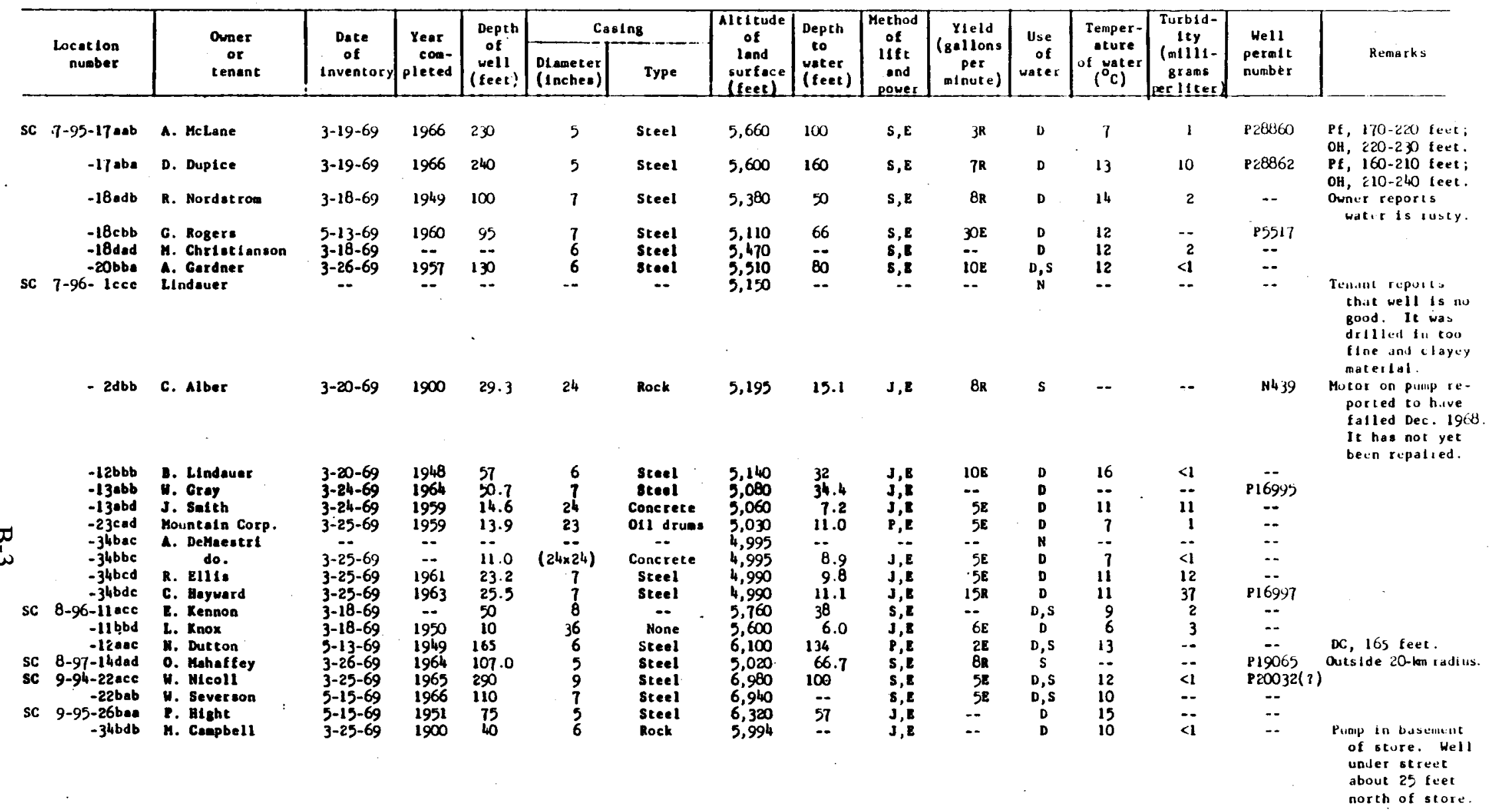


- -Records of Belected uells, Rullson project area, Garfield and Mesa Countles, Colorado--Continued

\begin{tabular}{|c|c|c|c|c|c|c|c|c|c|c|c|c|c|c|c|c|}
\hline \multirow{2}{*}{\multicolumn{2}{|c|}{$\begin{array}{l}\text { Location } \\
\text { neunber }\end{array}$}} & \multirow{2}{*}{$\begin{array}{l}\text { Owner } \\
\text { or } \\
\text { tenant }\end{array}$} & \multirow{2}{*}{$\begin{array}{c}\text { Date } \\
\text { of } \\
\text { Inventory }\end{array}$} & \multirow{2}{*}{$\begin{array}{r}\text { Year } \\
\text { con- } \\
\text { pleted }\end{array}$} & \multirow{2}{*}{$\begin{array}{c}\text { Depth } \\
\text { of } \\
\text { vell } \\
\text { (feet) }\end{array}$} & \multicolumn{2}{|c|}{ Casing } & \multirow{2}{*}{$\begin{array}{c}\text { Altitude } \\
\text { of } \\
\text { land } \\
\text { surface } \\
\text { (feet) }\end{array}$} & \multirow{2}{*}{$\begin{array}{c}\text { Depth } \\
\text { to } \\
\text { water } \\
\text { (feet) }\end{array}$} & \multirow{2}{*}{$\begin{array}{c}\text { Method } \\
\text { of } \\
\text { lift } \\
\text { and } \\
\text { power }\end{array}$} & \multirow{2}{*}{$\begin{array}{c}\text { yield } \\
\text { (gallons } \\
\text { per } \\
\text { minute) }\end{array}$} & \multirow{2}{*}{$\begin{array}{c}\text { Use } \\
\text { of } \\
\text { water }\end{array}$} & \multirow{2}{*}{$\begin{array}{l}\text { Temper- } \\
\text { ature } \\
\text { of water } \\
\left({ }^{\circ} \mathrm{C}\right)\end{array}$} & \multirow{2}{*}{$\begin{array}{c}\text { Turbid- } \\
\text { 1ty } \\
\text { (milli- } \\
\text { grame } \\
\text { eeritter) }\end{array}$} & \multirow{2}{*}{$\begin{array}{l}\text { Well } \\
\text { pernt } \\
\text { number }\end{array}$} & \multirow[b]{2}{*}{ Remarks } \\
\hline & & & & & & $\begin{array}{l}\text { D1 ameter } \\
\text { (1nches) }\end{array}$ & Type & & & & & & & & & \\
\hline sc & $9-0.75-35$ abc & T. Young & $5-20-69$ & 1964 & 765 & 7 & Steel & 6,100 & 55 & S,E & $50 E$ & D & -- & -- & PF6238 & $\begin{array}{l}\text { DC, } 765 \text { feet. } \\
\text { Pf, 175- } \\
200 \text { feet, } \\
405-510 \text { feet, } \\
\text { and } 565- \\
765 \text { feet. }\end{array}$ \\
\hline \multirow[t]{2}{*}{ sc } & $10-95-2 a a b$ & Unknown & 4- $3-69$ & -- & 35 & $\cdots$ & $-\cdot$ & 6,240 & -- & -- & -- & N & - & - & -- & $\begin{array}{l}\text { Pump bad. Tenan } \\
\text { haul Ing water. }\end{array}$ \\
\hline & $-2 b a a$ & H. Castle & 4- 3-69 & 1964 & 185 & 5 & Steel & 6,245 & 138 & $J, \Sigma$ & $12 R$ & D & -- & -- & P21409 & \\
\hline
\end{tabular}


(Adapted from Hurr, and others, 1969, and Iarson and Beetem, 1970.)

Location number: See text for spring-numbering system. Date of inventory: Date of inventory and yield measurement.

Altitude of land surface: Altitude of point of discharge, estimated from $7 \frac{1}{2}-m i n u t e$ quadrangle topographic maps, is given in feet above mean sea level.
Yield: $R$, reported; $E$, estimated.

Use of water: C, commercial; $D$, domest1C; I, Irrigation; $M$, munlcipal; S, stock.

Improvements: $B$, box; $N$, none; $P$, plpe; $U$, undetermined.

Temperature: Recorded to nearest $1^{\circ} \mathrm{C}$.

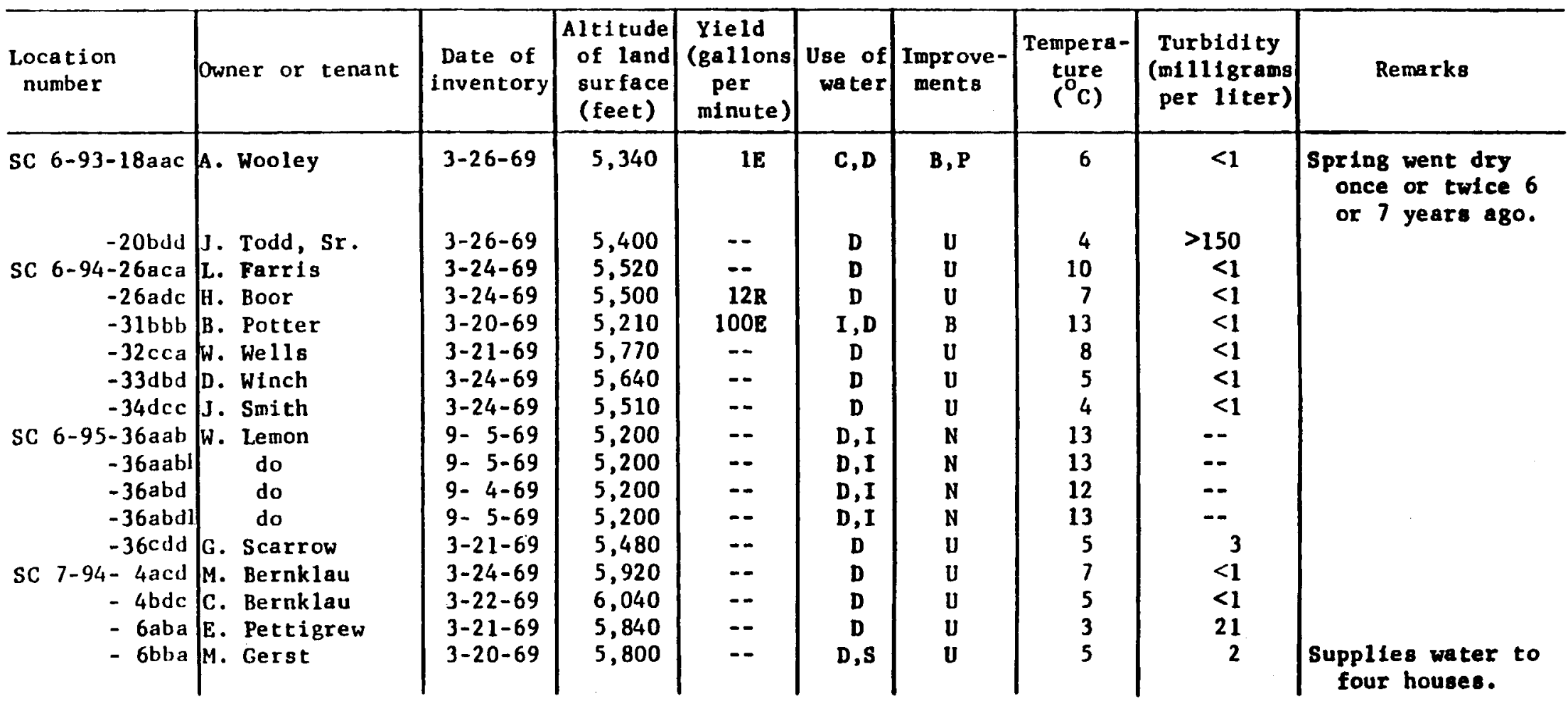


Records of selected springs, Rulison project area, Garfield and Mesa Countles, Colorado--Continued

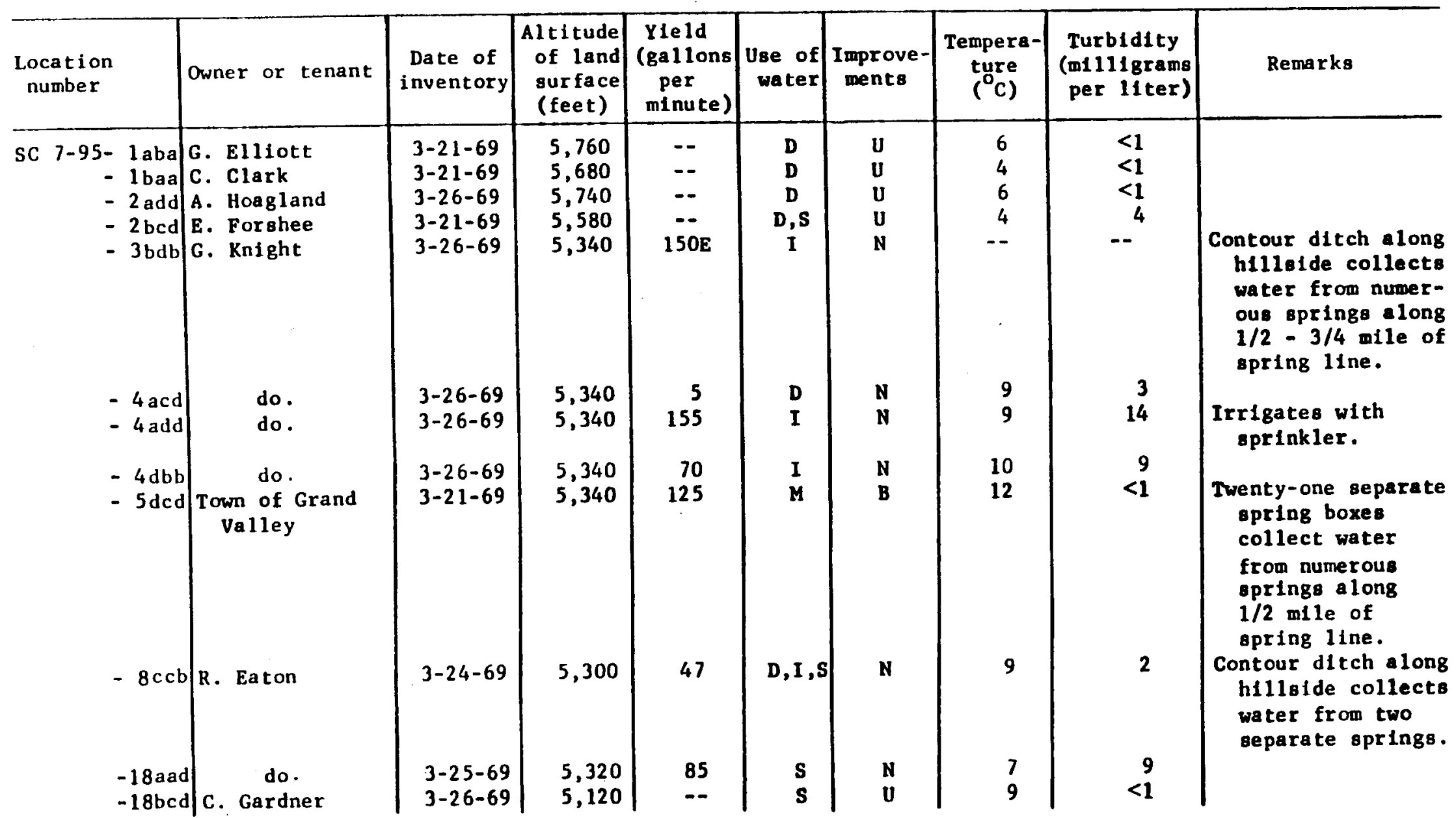


Records of selected springs, Ruli son profect area, Garfield and Mesa Counties, Colorado--Continued

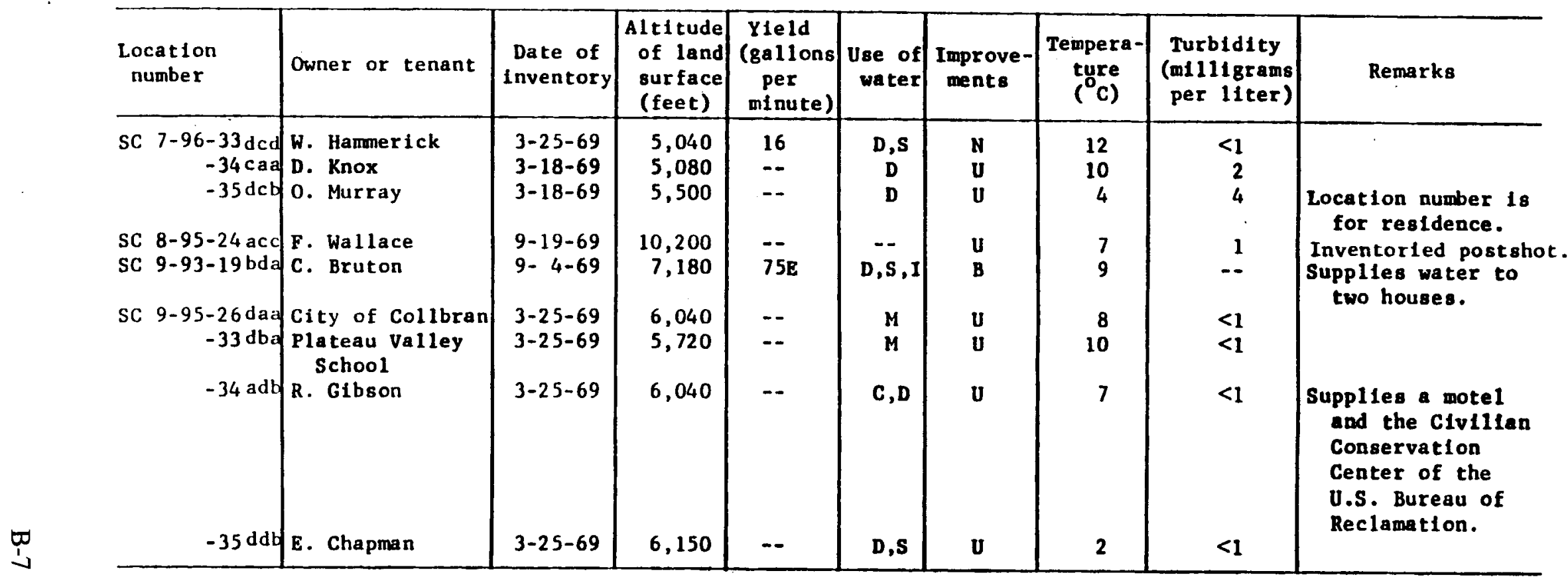




\section{Appendix C \\ Chemical Analyses of Groundwater and Surface Water, Project Rulison Area}




\begin{tabular}{|c|c|c|c|c|c|c|c|c|c|c|c|c|c|c|c|c|c|c|c|c|c|c|c|c|c|c|c|c|c|c|c|}
\hline taste & 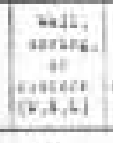 & 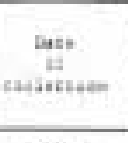 & $\begin{array}{l}\text { susace } \\
\text { inte, }\end{array}$ & \begin{tabular}{l} 
Ais \\
\hdashline$i$ ini
\end{tabular} & int & $\mid \begin{array}{l}m \\
\cdots \\
m\end{array}$ & $\sin _{i=0}$ & $\begin{array}{l}* 4: \\
\cdots \\
(4)\end{array}$ & 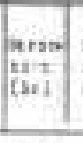 & $\begin{array}{l}\text { non: } \\
\text { inis. }\end{array}$ & 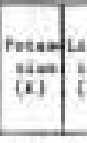 & (ش): & 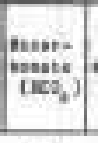 & 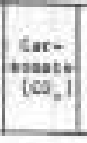 & 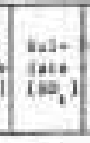 & $\begin{array}{l}\text { aus: } \\
\text { ratio } \\
\text { iai }\end{array}$ & 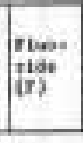 & wis & mo; & (16) & Fin & at: & itii & 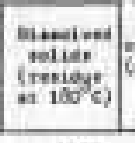 & 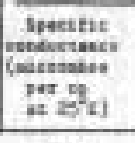 & 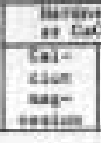 & 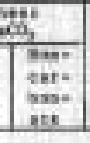 & $n$ & 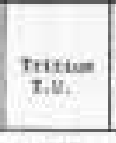 & 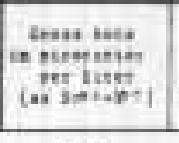 & 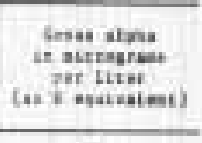 \\
\hline 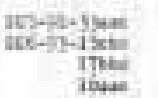 & $\frac{\pi}{i}$ & 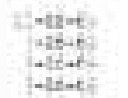 & $\frac{1}{\square}$ & $\begin{array}{l}c=1 \\
\ddot{*}\end{array}$ & $3^{\circ}$ & $\frac{c .1}{\ddot{z}}$ & $\begin{array}{l}\pi \\
\# \\
z\end{array}$ & $\underset{z}{*}$ & 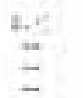 & \pm & $\begin{array}{l} \pm: 34 \\
z\end{array}$ & $\stackrel{0.1}{=}$ & $\frac{4}{3}$ & & $\stackrel{76 t}{\rightleftarrows}$ & $\begin{array}{l} \pm \\
z \\
z\end{array}$ & $\begin{array}{l}\text { s.y } \\
\ddot{Z}\end{array}$ & $=$ & $\frac{0.01}{z}$ & c.z & $\begin{array}{l}0.4 \\
z \\
z\end{array}$ & $\begin{array}{l}0.24 \\
\square \\
=\end{array}$ & $0 . z$ & $\frac{76]}{\pi}$ & 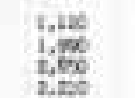 & $=$ & "y. & 4 & $\equiv$ & $\begin{array}{l}a: 1 \\
\because\end{array}$ & I \\
\hline 100 & $\frac{\pi}{6}$ & $\begin{array}{l}\text { thet } \\
\text { thes }\end{array}$ & $z$ & $=$ & E: & ": & 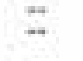 & $=$ & $=$ & 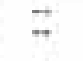 & $=$ & $=$ & 翟 & $\frac{7}{2}$ & 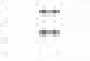 & $\overline{-}$ & $\ddot{z}$ & $=$ & 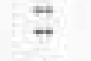 & $=$ & $\ddot{z}$ & $\because$ & 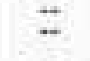 & $\ddot{*}$ & $\frac{2,56}{3,60}$ & $z$ & $\ddot{*}$ & 48 & $=$ & $z$ & 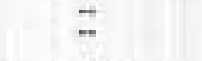 \\
\hline$\frac{\operatorname{man}}{\operatorname{man}}$ & $\frac{1}{2}$ & thet: & \pm & 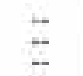 & 5 & $z$ & 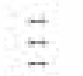 & $\bar{z}$ & $\equiv$ & $z$ & $=$ & $\Xi$ & $\frac{a y}{y b}$ & & 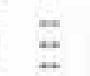 & : & $\ddot{z}$ & $\equiv$ & Z & 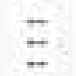 & $\equiv$ & $\Xi$ & $\bar{z}$ & $\approx$ & 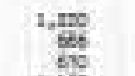 & $z$ & $\ddot{z}$ & 74 & $\equiv$ & 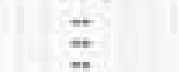 & Z \\
\hline ister & $\frac{i}{i}$ & thet & 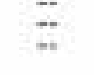 & $\ddot{z}$ & 典 & $\ddot{z}$ & $\bar{z}$ & $\ddot{z}$ & $\ddot{z}$ & $\ddot{z}$ & $=$ & 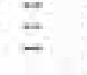 & tit & $\frac{1}{2}$ & $\ddot{z}$ & $\ddot{z}$ & $\ddot{z}$ & 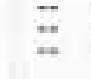 & 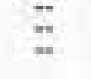 & $\ddot{z}$ & $\ddot{z}$ & 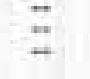 & $\ddot{z}$ & $\ddot{z}$ & t, twe & $\exists$ & $z$ & 78 & $\Xi$ & $\ddot{z}$ & $\bar{z}$ \\
\hline 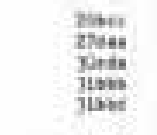 & 8 & tat & $\ddot{z}$ & 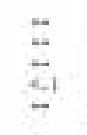 & ti & $\ddot{z}$ & $\bar{z}$ & $\ddot{z}$ & $\equiv$ & $\ddot{z}$ & $\ddot{z}$ & $\sum_{i=1}$ & 然 & & ii & $\ddot{z}$ & $z$ & $\ddot{7}$ & $\ddot{\ddot{z}}$ & $\underline{z}$ & $\underline{z}$ & $\underline{z}$ & $\frac{z}{2}$ & $\ddot{z}$ & 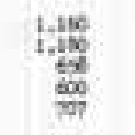 & $\ddot{z}$ & $\ddot{z}$ & $\begin{array}{l}y .2 \\
7.4\end{array}$ & $\underset{-0}{-\infty}$ & $\frac{6.1}{\vec{B}, 2}$ & $\begin{array}{l}\text { G.t } \\
\text { in } \\
\text { II }\end{array}$ \\
\hline 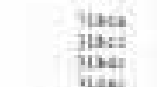 & 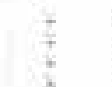 & +10 & 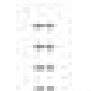 & $\equiv$ & 霄 & $\ddot{z}$ & $\ddot{z}$ & 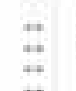 & 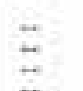 & $\equiv$ & $z$ & $\bar{z}$ & 背 & & $\ddot{z}$ & $=$ & $=$ & $\ddot{z}$ & $\ddot{z}$ & $z$ & $z$ & $=$ & $\approx$ & $\ddot{z}$ & 7 & $\ddot{z}$ & $\Xi$ & If & 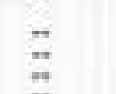 & 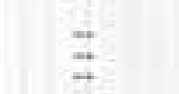 & \\
\hline Hat: & i & seltit & : & $\approx$ & 到 & $=$ & $=$ & $ت$ & $\because$ & 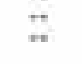 & 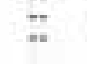 & :- & 㕍 & & $\approx$ & 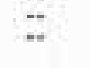 & $=$ & : & 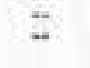 & $\ddot{z}$ & $=$ & $\ddot{7}$ & $=$ & $\approx$ & 1,710 & 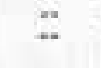 & $=$ & 4 & $\ddot{a x}$ & 3. & a.c \\
\hline 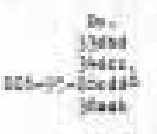 & $\begin{array}{l}3 \\
3 \\
3 \\
\vdots \\
\vdots\end{array}$ & 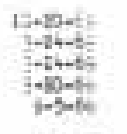 & $\frac{n}{\ddot{z}}$ & $\begin{array}{l}5 \\
4 \\
4\end{array}$ & 4 & $\begin{array}{l}\ddot{4} \\
\ddot{z}\end{array}$ & $\frac{4}{a}$ & $\begin{array}{l} \pm \\
\because \\
z \\
z\end{array}$ & $\ddot{z}$ & $\frac{1 !}{\frac{12}{x}}$ & $\because:$ & $=1$ & 細 & t. & $\stackrel{\ddot{*}}{\ddot{1}}$ & $\begin{array}{l}\text { :" } \\
\ddot{z}\end{array}$ & $\frac{a}{a}$ & ${ }^{1}=$ & $\begin{array}{l}\text { s.m } \\
\dddot{\pi} \\
\text { con }\end{array}$ & $\frac{\pi}{0}$ & $\ddot{z}$ & $\ddot{z}$ & $\begin{array}{l}\text { en } \\
\ddot{4}\end{array}$ & 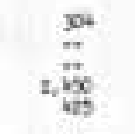 & 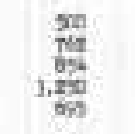 & 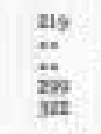 & 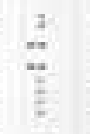 & it. & $\begin{array}{l}a x \\
a x \\
a x\end{array}$ & 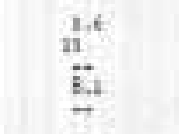 & $\frac{4}{3}$ \\
\hline 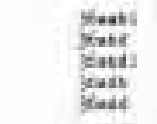 & $\begin{array}{l}1 \\
1 \\
\vdots\end{array}$ & $\sum_{1 \rightarrow 0}^{+50}$ & $\ddot{z}$ & $\underline{z}$ & in & $\begin{array}{l}z \\
z\end{array}$ & 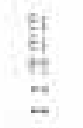 & 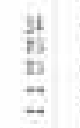 & $\ddot{z}$ & :: & $\begin{array}{l}z \\
z \\
z\end{array}$ & $z$ & 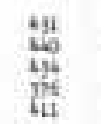 & & 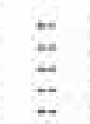 & $=$ & $\equiv$ & $\ddot{z}$ & $z$ & $\begin{array}{l}z \\
z\end{array}$ & $\begin{array}{l}z \\
z\end{array}$ & $=$ & $=$ & 薄 & $\begin{array}{l}\text { os } \\
\text { if } \\
\text { w }\end{array}$ & $\begin{array}{l}39 \\
30 \\
30 \\
=\end{array}$ & $\underline{2}$ & if & 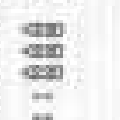 & $z$ & 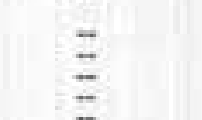 \\
\hline sitis & $\vdots$ & 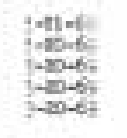 & $\begin{array}{l}\frac{11}{\ddot{z}} \\
\stackrel{2}{*}\end{array}$ & $\frac{z_{1}}{2}$ & 怠 & $\frac{3}{5}$ & 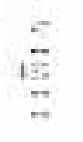 & $\frac{\pi}{a}$ & $\stackrel{4}{\ddot{a}}$ & $\frac{\theta}{\ddot{*}}$ & $\frac{4}{2}$ & $\begin{array}{l}a n \\
=\end{array}$ & 亲 & $\vdots$ & $\frac{1}{2}$ & ב.9. & $\frac{1}{1}$ & 3 & $\sum_{0}^{\cos }$ & $\frac{n}{8}$ & $\begin{array}{l}\frac{01}{15} \\
=\end{array}$ & $\begin{array}{l}\text { cat } \\
\vdots \\
=\end{array}$ & $\frac{.07}{16}$ & $\frac{m}{3}$ & 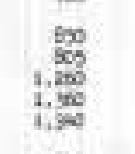 & $\frac{2}{2 \pi}$ & $\frac{z}{2}$ & 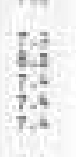 & 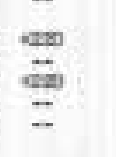 & $\because 4$ & 产 \\
\hline $\operatorname{mot}_{0}$ & $\vdots$ & tiont & $\frac{z}{1}$ & $\frac{\pi}{4}$ & 4 & $\ddot{z}$ & $\frac{\pi}{3}$ & $\begin{array}{l}\ddot{z} \\
z\end{array}$ & $\frac{\pi}{4}$ & $\ddot{z}$ & \pm & $\ddot{z}$ & 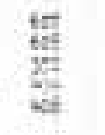 & & $\underline{z}$ & $\ddot{\ddot{I}}$ & $\ddot{z}$ & $\because$ & 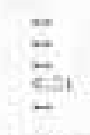 & 范 & 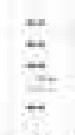 & $\frac{\pi}{a}$ & $\frac{1}{9}$ & $\ddot{z}$ & 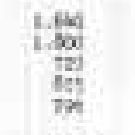 & $\frac{\pi}{4}$ & $\ddot{z}$ & th & $\ddot{z}$ & 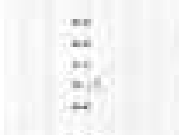 & $\ddot{z}$ \\
\hline 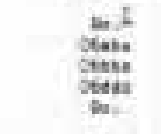 & $\begin{array}{l}3 \\
\vdots \\
\vdots \\
\vdots\end{array}$ & 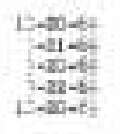 & 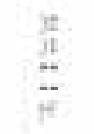 & $\frac{1}{3}$ & 4 & $\frac{9}{2}$ & $\frac{3}{Z}$ & $\frac{4}{2}$ & $\vec{z}$ & $\frac{4}{\frac{5}{5}}$ & $\frac{3}{3}$ & $\frac{a}{E}$ & 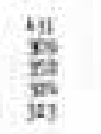 & & $\frac{k}{n}$ & $\begin{array}{l}\text { te.s } \\
\frac{7}{7.2}\end{array}$ & $\frac{3}{7}$ & $\frac{30}{2.0}$ & 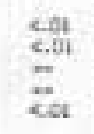 & $\begin{array}{l}\text { aี } \\
\ddot{0} \\
\text { : }\end{array}$ & a & $\frac{a}{a}$ & $\frac{10}{10}$ & 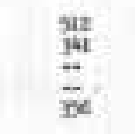 & 紫 & 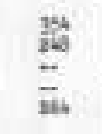 & $\stackrel{1}{3}$ & 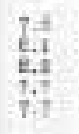 & $\begin{array}{l}a x \\
\frac{a x}{a x} \\
a x\end{array}$ & 迹: & $\frac{4^{6.6}}{8.9}$ \\
\hline 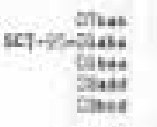 & $\begin{array}{l}\vdots \\
\vdots \\
\vdots\end{array}$ & 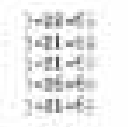 & $\ddot{z}$ & $\equiv$ & 嵲 & $\equiv$ & $\ddot{z}$ & $\ddot{z}$ & $\ddot{z}$ & $\exists$ & $\begin{array}{l}\ddot{Z} \\
\ddot{2} .2\end{array}$ & $z_{01}$ & 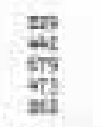 & & 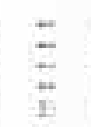 & $\begin{array}{l}\ddot{z} \\
\ddot{z}\end{array}$ & $\begin{array}{l}z \\
\ddot{z}\end{array}$ & $\bar{z}$ & $\ddot{z}$ & $\begin{array}{l}\ddot{z} \\
\ddot{q}\end{array}$ & $\ddot{z}$ & $\ddot{z}$ & $\ddot{z}$ & $\ddot{z}$ & 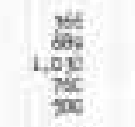 & $\begin{array}{l}z \\
\text { as }\end{array}$ & $\ddot{z}$ & 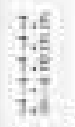 & $\ddot{z}$ & $\underline{z}$ & $\bar{z}$ \\
\hline$\frac{1}{x+4}$ & $\vdots$ & $\frac{x-4}{-4 t}$ & $\frac{\pi}{n}$ & $\begin{array}{l}4 \\
4 \\
4\end{array}$ & $\frac{4}{4}$ & int & $\begin{array}{l}\text { a } \\
z \\
z\end{array}$ & 莫 & $\frac{4}{*}$ & $\frac{1 x}{\frac{1 x}{2}}$ & 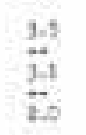 & $=$ & 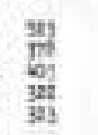 & & $\frac{2}{-10}$ & $\begin{array}{l}1 . \\
a .9 \\
\ddot{3},\end{array}$ & $\frac{2}{3}$ & $\begin{array}{l}e .3 \\
.7 \\
a .8\end{array}$ & $\begin{array}{l}4.31 \\
\frac{8 . a t}{201}\end{array}$ & $\frac{0 s}{\ddot{c o t}}$ & $\frac{a}{a}$ & cait & $\frac{x}{30}$ & 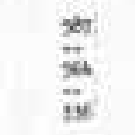 & 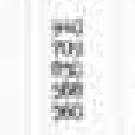 & $\sum_{i=2}^{\infty}$ & $\ddot{z}$ & it & $\frac{a n}{a x}$ & $\begin{array}{l}26 \\
50\end{array}$ & $\frac{17}{9,4}$ \\
\hline 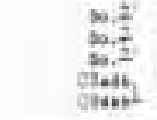 & $\begin{array}{l}\frac{1}{8} \\
\frac{1}{8} \\
8\end{array}$ & 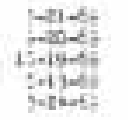 & $z$ & $\begin{array}{l}z \\
z \\
z\end{array}$ & $\begin{array}{l}\ddot{4} \\
\ddot{4}\end{array}$ & $\ddot{\ddot{a}}$ & $\ddot{z}$ & ii & $\ddot{z}$ & $\ddot{z}$ & $\frac{\pi}{3}$ & $\bar{z}$ & $\frac{3}{4 \pi}$ & $\begin{array}{l}0 \\
\vdots \\
\vdots \\
\vdots\end{array}$ & $\bar{z}$ & $\ddot{z}$ & $\equiv$ & $\frac{\pi}{4}$ & $\ddot{z}$ & $z$ & $z$ & $\ddot{z}$ & 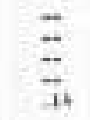 & $\frac{0}{40}$ & $\frac{76}{1,40}$ & in & $\frac{\hbar}{t}$ & $\frac{4}{2}$ & 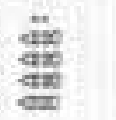 & 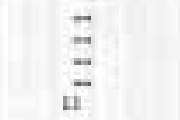 & z \\
\hline
\end{tabular}




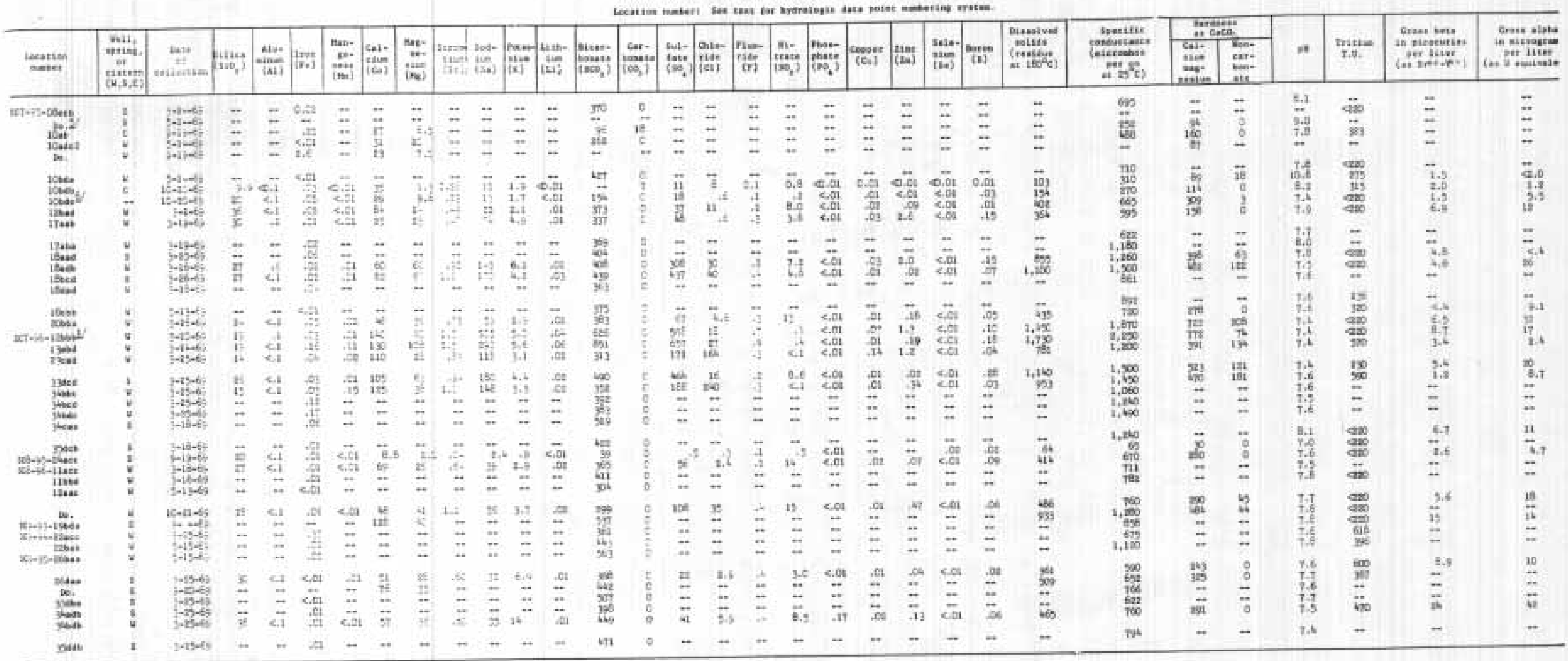

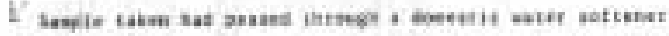

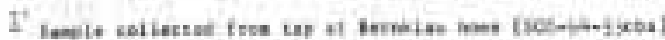

I setate calarinatim.

Aiter thisuastan

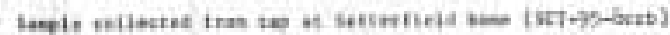

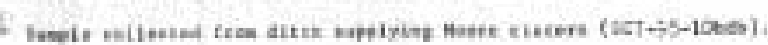


(Chemical analyses in milligrams per liter.)

\begin{tabular}{|c|c|c|c|c|c|c|c|c|c|c|c|c|c|c|c|}
\hline \multirow[b]{2}{*}{ Station name } & \multirow[b]{2}{*}{$\begin{array}{c}\text { Altitude } \\
\text { (feet } \\
\text { above msi }\end{array} \mid$} & \multirow[b]{2}{*}{ Date } & \multirow[b]{2}{*}{$\mid \begin{array}{l}\text { Time } \\
\text { (moun- } \\
\text { tain } \\
\text { day- } \\
1 \text { ight) }\end{array}$} & \multirow[b]{2}{*}{$\begin{array}{c}\text { Tem- } \\
\text { per- } \\
\text { atture } \\
\text { ( } \mathrm{C} \text { C) }\end{array}$} & \multirow[b]{2}{*}{$\begin{array}{l}\text { Cal- } \\
\text { clum } \\
(\mathrm{Ca})\end{array}$} & \multirow{2}{*}{$\mid \begin{array}{c}\text { Mag- } \\
\text { ne- } \\
\text { sumu } \\
\text { (Mg) }\end{array}$} & \multirow{2}{*}{$\begin{array}{c}\mathrm{B1}- \\
\text { car- } \\
\text { bon- } \\
\text { ate } \\
\left(\mathrm{HCO}_{3}\right)\end{array}$} & \multirow[b]{2}{*}{$\begin{array}{l}\text { Car- } \\
\text { bon- } \\
\text { ate } \\
\left(\mathrm{CO}_{3}\right)\end{array}$} & \multicolumn{2}{|c|}{$\begin{array}{l}\text { Hardnegs } \\
\text { as Caco } \\
\end{array}$} & \multirow{2}{*}{\begin{tabular}{|c|} 
Dis- \\
solved \\
sollds \\
(rest- \\
due \\
at \\
$180^{\circ} \mathrm{C}$ ) \\
\end{tabular}} & \multirow{2}{*}{\begin{tabular}{|c|} 
Specificic \\
conduct- \\
ance \\
(mitro- \\
mhos per \\
cm at 25\%
\end{tabular}} & \multirow[b]{2}{*}{$\mathrm{pH}$} & \multirow[b]{2}{*}{$\left|\begin{array}{l}\text { Tur- } \\
\text { bld- } \\
\text { ity }\end{array}\right|$} & \multirow[b]{2}{*}{$\begin{array}{l}\text { Trit- } \\
\text { Lum } \\
\text { (T.u. }) 1 /\end{array}$} \\
\hline & & & & & & & & & $\begin{array}{l}\text { cal- } \\
\text { cium } \\
\text { mag- } \\
\text { ne- } \\
\text { sium } \\
\end{array}$ & \begin{tabular}{|l|} 
Non- \\
car- \\
bon- \\
ate
\end{tabular} & & & & & \\
\hline $\begin{array}{l}\text { Colorado River at New Castle } \\
\text { Colorado River at New Castle } \\
\text { East Mamm Creek near Rifle } \\
\text { Middle Mamm Creek near Rifle } \\
\text { West Mamm Creek near Rifle }\end{array}$ & $\begin{array}{l}5,515 \\
5,515 \\
6,220 \\
6,830 \\
7,080\end{array}$ & $\begin{array}{r}8-26-69 \\
10-19-69 \\
9-2-69 \\
8-27-69 \\
8-27-69\end{array}$ & $\begin{array}{l}1225 \\
1600 \\
1500 \\
1235 \\
1145\end{array}$ & $\begin{array}{r}19.0 \\
8.0 \\
27.0 \\
19.5 \\
13.5\end{array}$ & $\begin{array}{l}71 \\
-- \\
51 \\
44 \\
62\end{array}$ & $\begin{array}{l}14 \\
- \\
60 \\
21 \\
31\end{array}$ & $\begin{array}{l}161 \\
--1 \\
671 \\
261 \\
360\end{array}$ & $\begin{array}{l}0 \\
0- \\
0 \\
0 \\
0\end{array}$ & $\begin{array}{l}235 \\
-- \\
374 \\
197 \\
282\end{array}$ & $\begin{array}{l}103 \\
-- \\
0 \\
0 \\
0\end{array}$ & $\begin{array}{r}534 \\
-- \\
1,050 \\
237 \\
369\end{array}$ & $\begin{array}{r}898 \\
1, \overline{460} \\
450 \\
648\end{array}$ & $\begin{array}{l}7.3 \\
-8.1 \\
7.9 \\
7.6\end{array}$ & $\begin{array}{l}6 \\
-- \\
-- \\
4\end{array}$ & $\begin{array}{l}368 \\
<220 \\
<220 \\
<220 \\
<220\end{array}$ \\
\hline $\begin{array}{l}\text { Mamm Creek near Rifle } \\
\text { Beaver Creek near Rifle } \\
\text { Beaver Creek near Rifle } \\
\text { Cache Creek near Rulison } \\
\text { Battlement Reservolr near Grand Valley }\end{array}$ & $\begin{array}{r}5,610 \\
6,685 \\
6,685 \\
5,950 \\
10,200\end{array}$ & $\begin{array}{l}8-27-69 \\
3-24-69 \\
9-20-69 \\
8-27-69 \\
9-3-69\end{array}$ & $\begin{array}{l}1300 \\
1209 \\
1025 \\
1000\end{array}$ & $\begin{array}{r}27.5 \\
1.0 \\
10.0 \\
13.5 \\
7.0\end{array}$ & $\begin{array}{l}36 \\
21 \\
7.1\end{array}$ & $\begin{array}{r}8.0 \\
4.5 \\
.8\end{array}$ & $\begin{array}{r}173 \\
101 \\
35\end{array}$ & $\begin{array}{l}0 \\
0 \\
0\end{array}$ & $\begin{array}{r}124 \\
71 \\
21\end{array}$ & $\begin{array}{l}0 \\
0 \\
0\end{array}$ & $\begin{array}{r}149 \\
81 \\
53\end{array}$ & $\begin{array}{r}282 \\
171 \\
60\end{array}$ & $\begin{array}{l}8.1 \\
7.0 \\
7.0\end{array}$ & $\begin{array}{r}15 \\
5 \\
--\end{array}$ & $\begin{array}{r}<220 \\
263 \\
336\end{array}$ \\
\hline $\begin{array}{l}\text { Battlement Creek near Morrisania } \\
\text { Battlement Creek near Morrisania } \\
\text { Tributary of Battlement Creek near }\end{array}$ & $\begin{array}{l}7,760 \\
7,760\end{array}$ & $\begin{array}{l}8-28-69 \\
9-20-69\end{array}$ & $\begin{array}{l}0815 \\
1355\end{array}$ & $\begin{array}{l}9.5 \\
9.5\end{array}$ & $\begin{array}{l}10 \\
12\end{array}$ & $\begin{array}{l}2.5 \\
2.6\end{array}$ & $\begin{array}{l}55 \\
59\end{array}$ & $\begin{array}{l}0 \\
0\end{array}$ & $\begin{array}{l}36 \\
41\end{array}$ & $\begin{array}{l}0 \\
0\end{array}$ & $\begin{array}{l}74 \\
41\end{array}$ & $\begin{array}{r}96 \\
100\end{array}$ & $\begin{array}{l}7.1 \\
7.4\end{array}$ & $\begin{array}{r}10 \\
2\end{array}$ & $\begin{array}{r}229 \\
<220\end{array}$ \\
\hline 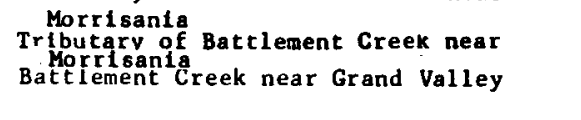 & $\begin{array}{l}7,880 \\
7,880 \\
6,630\end{array}$ & $\begin{array}{l}8-27-69 . \\
9-20-69 \\
8-27-69\end{array}$ & $\begin{array}{l}1500 \\
1400 \\
1425\end{array}$ & $\begin{array}{r}17.0 \\
16.5 \\
8.0\end{array}$ & $\begin{array}{l}36 \\
36 \\
17\end{array}$ & $\begin{array}{l}11 \\
11 \\
5.0\end{array}$ & $\begin{array}{r}200 \\
208 \\
89\end{array}$ & $\begin{array}{l}0 \\
0 \\
0\end{array}$ & $\begin{array}{r}135 \\
135 \\
63\end{array}$ & $\begin{array}{l}0 \\
0 \\
0\end{array}$ & $\begin{array}{l}147 \\
178 \\
110\end{array}$ & $\begin{array}{l}322 \\
338 \\
150\end{array}$ & $\begin{array}{l}7.3 \\
8.0 \\
6.8\end{array}$ & $\begin{array}{r}<1 \\
7 \\
2\end{array}$ & $\begin{array}{r}<220 \\
<220 \\
258\end{array}$ \\
\hline $\begin{array}{l}\text { Battlement Creek near Grand Valley } \\
\text { Battlement Greek near Grand Valley } \\
\text { Spring Creek near Grand Valley } \\
\text { colorado River near DeBeque } \\
\text { Colorado River near DeBeque }\end{array}$ & $\begin{array}{l}6,630 \\
6,630 \\
5,080 \\
4,940 \\
4,940\end{array}$ & $\begin{array}{r}9-20-69 \\
10-19-69 \\
8-27-69 \\
8-26-69 \\
9-20-69\end{array}$ & $\begin{array}{l}1320 \\
1420 \\
1610 \\
1615 \\
1515\end{array}$ & $\begin{array}{r}11.0 \\
4.0 \\
22.0 \\
23.5 \\
17.0\end{array}$ & $\begin{array}{l}.23 \\
29 \\
67 \\
105\end{array}$ & $\begin{array}{l}7.3 \\
38 \\
15 \\
16\end{array}$ & $\begin{array}{l}126 \\
-- \\
334 \\
157 \\
166\end{array}$ & $\begin{array}{l}0 \\
-- \\
0 \\
0 \\
0\end{array}$ & $\begin{array}{l}88 \\
-- \\
229 \\
229 \\
328\end{array}$ & $\begin{array}{r}0 \\
-- \\
100 \\
192\end{array}$ & $\begin{array}{l}104 \\
-8 \\
471 \\
537 \\
601\end{array}$ & $\begin{array}{r}212 \\
7- \\
790 \\
882 \\
1,030\end{array}$ & $\begin{array}{l}8.0 \\
-- \\
7.9 \\
6.5 \\
8.1\end{array}$ & $\begin{array}{c}2 \\
- \\
1 \\
10 \\
10\end{array}$ & $\begin{array}{l}<220 \\
<220 \\
<220 \\
335 \\
<220\end{array}$ \\
\hline $\begin{array}{l}\text { Colorado RIver near DeBeque } \\
\text { Vega Reservol near Collbran } \\
\text { plateau Creek near Collbran } \\
\text { Road Gulch near Collbran } \\
\text { Buzzard Creek near Collbran }\end{array}$ & $\begin{array}{l}4,940 \\
7,906 \\
7,130 \\
7,400 \\
6,955\end{array}$ & $\begin{array}{r}10-19-69 \\
8-26-69 \\
8-27-69 \\
8-28-69 \\
8-26-69\end{array}$ & $\begin{array}{l}1200 \\
1945 \\
0755 \\
110 \\
1830\end{array}$ & $\begin{array}{l}18.0 \\
14.0 \\
12.0 \\
18.5 \\
22.0\end{array}$ & $\begin{array}{l}-\overline{18} \\
17 \\
51 \\
51\end{array}$ & $\begin{array}{l}-- \\
3.2 \\
4.2 \\
10 \\
25\end{array}$ & $\begin{array}{r}-\overline{81} \\
83 \\
295 \\
338\end{array}$ & $\begin{array}{l}-- \\
0 \\
0 \\
0 \\
0\end{array}$ & $\begin{array}{r}-- \\
58 \\
60 \\
168 \\
230\end{array}$ & $\begin{array}{r}-- \\
0 \\
0 \\
0 \\
0\end{array}$ & $\begin{array}{r}-- \\
56 \\
57 \\
276 \\
383\end{array}$ & $\begin{array}{l}-2 \\
124 \\
121 \\
475 \\
565\end{array}$ & $\begin{array}{l}-- \\
6.7 \\
6.8 \\
7.9 \\
7.8\end{array}$ & $\begin{array}{r}7 \\
2 \\
2 \\
15 \\
10\end{array}$ & $\begin{array}{r}288 \\
230 \\
240 \\
<220 \\
430\end{array}$ \\
\hline $\begin{array}{l}\text { Buzzard Creek near Collbran } \\
\text { Brush Creek near Collbran } \\
\text { Hawhurst Creek near Collbran } \\
\text { Hawxhurst Creek near Collbran } \\
\text { Kimball Creek near Collbran }\end{array}$ & $\begin{array}{l}6,955 \\
8,183 \\
6,560 \\
6,560 \\
6,880\end{array}$ & $\begin{array}{l}9-20-69 \\
8-26-69 \\
8-26-69 \\
9-20-69 \\
8-26-69\end{array}$ & $\begin{array}{l}1800 \\
1905 \\
1800 \\
1655 \\
1735\end{array}$ & $\begin{array}{l}14.5 \\
16.0 \\
19.0 \\
14.5 \\
17.0\end{array}$ & $\begin{array}{l}76 \\
51 \\
51 \\
76 \\
67\end{array}$ & $\begin{array}{l}18 \\
13 \\
29 \\
30 \\
20\end{array}$ & $\begin{array}{l}322 \\
248 \\
430 \\
443 \\
433\end{array}$ & $\begin{array}{l}0 \\
0 \\
0 \\
0 \\
0\end{array}$ & $\begin{array}{l}264 \\
181 \\
247 \\
313 \\
250\end{array}$ & $\begin{array}{l}0 \\
0 \\
0 \\
0 \\
0\end{array}$ & $\begin{array}{l}335 \\
201 \\
370 \\
404 \\
384\end{array}$ & $\begin{array}{l}580 \\
350 \\
605 \\
668 \\
610\end{array}$ & $\begin{array}{l}8.2 \\
7.9 \\
7.5 \\
7.9 \\
7.5\end{array}$ & $\begin{array}{r}2 \\
10 \\
2 \\
4 \\
41\end{array}$ & $\begin{array}{r}<220 \\
263 \\
354 \\
250 \\
<220\end{array}$ \\
\hline $\begin{array}{l}\text { Kimball Creek near Collbran } \\
\text { Plateau Creek near Cameo } \\
\text { Plateau Creek near Cameo } \\
\text { Plateau Creek near Cameo }\end{array}$ & $\begin{array}{l}6,880 \\
4,836 \\
4,836 \\
4,836\end{array}$ & $\begin{array}{r}9-20-69 \\
8-28-69 \\
9-20-69 \\
10-19-69\end{array}$ & $\begin{array}{l}1630 \\
0955 \\
1545 \\
1100\end{array}$ & $\begin{array}{l}12.5 \\
18.5 \\
17.0 \\
16.0\end{array}$ & $\begin{array}{r}105 \\
41 \\
37 \\
--\end{array}$ & $\begin{array}{l}20 \\
38 \\
35 \\
-\end{array}$ & $\begin{array}{l}481 \\
411 \\
385\end{array}$ & $\begin{array}{l}0 \\
0 \\
0 \\
--\end{array}$ & $\begin{array}{l}345 \\
259 \\
237 \\
--\end{array}$ & $\begin{array}{l}0 \\
0 \\
0 \\
---\end{array}$ & $\begin{array}{l}425 \\
485 \\
418 \\
--\end{array}$ & $\begin{array}{l}708 \\
780 \\
712 \\
--\end{array}$ & $\begin{array}{l}7.9 \\
8.1 \\
8.1 \\
--\end{array}$ & $\begin{array}{r}2 \\
2 \\
8 \\
--\end{array}$ & $\begin{array}{l}<220 \\
<220 \\
<220 \\
291\end{array}$ \\
\hline
\end{tabular}

1/ The tritium analyses were by liquid scintillation counting and the lowest detectable concentration by this method was 220 T.U. 


\section{Appendix D \\ Radiochemical Analyses of Spring, Well, and Stream Waters, Rulison Project Area}




\begin{tabular}{|c|c|c|c|c|c|c|c|c|c|c|c|c|c|c|c|c|c|c|c|}
\hline \multirow[b]{2}{*}{ Owner or tenant } & \multirow[b]{2}{*}{$\begin{array}{c}\text { Sample } \\
\text { point } \\
\text { number }\end{array}$} & \multicolumn{3}{|c|}{ Locetion } & \multicolumn{3}{|c|}{ Letitude N. } & \multicolumn{3}{|c|}{ Long I tude $N$. } & \multirow[b]{2}{*}{$\begin{array}{l}\text { Diotence froo } \\
\text { aurfece ground } \\
\text { zero, in allen } \\
\text { (kitometere) }\end{array}$} & \multirow[b]{2}{*}{$\begin{array}{c}\text { Date } \\
\text { of } \\
\text { collection }\end{array}$} & \multicolumn{2}{|c|}{ Irtitue } & \multicolumn{2}{|c|}{ Grose spha } & \multicolumn{2}{|c|}{ Groses bete } & \multirow[b]{2}{*}{ Remerks } \\
\hline & & 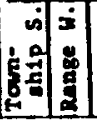 & 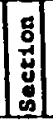 & \begin{tabular}{|l|} 
\\
\\
0 \\
0 \\
\end{tabular} & \begin{tabular}{|l|} 
\\
8 \\
8 \\
8
\end{tabular} & 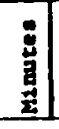 & 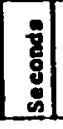 & $\begin{array}{l}8 \\
8 \\
8\end{array}$ & 总 & 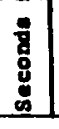 & & & $\mathrm{pci} / 1$ & Tu & $\begin{array}{c}(\mu g / 1 \\
0 \\
u \text { matural })\end{array}$ & {$\left[\begin{array}{c}(\mathrm{pC1} / 1 \\
0 \\
0 \mathrm{netura1}\end{array}\right]$} & $\begin{array}{c}(p C 1 / 1 \\
a 0 \\
s x-90) \\
y-90)\end{array}$ & $\begin{array}{c}(p c 1 / 1 \\
a \\
c=-137)\end{array}$ & \\
\hline Mrs. Betty Potter & 20 & 694 & 31 & NW & 39 & 29 & 20 & 107 & 56 & 12 & $5.7(9.2)$ & $\begin{array}{l}3-20-69 \\
4-10-70<\end{array}$ & $\begin{array}{r}<700 \\
<1,300\end{array}$ & $\begin{array}{l}<220 \\
<400\end{array}$ & $\begin{array}{l}12 \\
18\end{array}$ & $\begin{array}{l}3.9 \\
5.9\end{array}$ & $\begin{array}{l}8.4 \\
8.8\end{array}$ & ${ }_{9.1}^{11}$ & -- \\
\hline Carl Bernklau & 21 & 794 & 4 & NH & 39 & 28 & 09 & 107 & 53 & 45 & $5.1(8.2)$ & $\begin{array}{r}10-20-69 \\
4-10-70\end{array}$ & $\begin{array}{l}<960 \\
<960\end{array}$ & $\begin{array}{l}<300 \\
<300\end{array}$ & $\begin{array}{l}10 \\
10\end{array}$ & $\begin{array}{l}3.4 \\
3.5\end{array}$ & $\begin{array}{l}4.6 \\
4.3\end{array}$ & $\begin{array}{l}5.8 \\
4.8\end{array}$ & -- \\
\hline Town of Grand Valley & 22 & 795 & 5 & SE & 39 & 27 & 49 & 108 & 00 & 58 & $5.3(8.5)$ & $\begin{array}{c}3-21-69 \\
9-20-69 \\
10-19-69 \\
4-11-70<\end{array}$ & $\begin{array}{r}<700 \\
<960 \\
<960 \\
<1,300\end{array}$ & $\begin{array}{l}<220 \\
<300 \\
<300 \\
<400\end{array}$ & $\begin{array}{l}21 \\
-- \\
20\end{array}$ & $\begin{array}{l}6.8 \\
--. \\
6.7\end{array}$ & $\begin{array}{l}3.0 \\
-- \\
3.0\end{array}$ & $\begin{array}{l}3.7 \\
-. \\
3.4\end{array}$ & $\begin{array}{l}\text { Toun of Grand valley } \\
\text { weter oupply. }\end{array}$ \\
\hline ot 1s Murray & 23 & 796 & 35 & SE & 39 & 23 & 23 & 108 & 04 & 28 & $6.8(11)$ & $\begin{array}{l}3-18-69 \\
4-11-70<\end{array}$ & $\begin{array}{r}<700 \\
<1,300\end{array}$ & $\begin{array}{l}<220 \\
<400\end{array}$ & $\begin{array}{l}11 \\
45\end{array}$ & $15^{3.7}$ & $19^{6.7}$ & $24^{8.4}$ & -- \\
\hline Cecil Gardner & 24 & 795 & 18 & NH & 39 & 26 & 16 & 108 & 02 & 40 & $5.6(9.0)$ & $\begin{array}{l}3-26-69 \\
4-11-70<\end{array}$ & $\begin{array}{r}<700 \\
<1,300\end{array}$ & $\begin{array}{l}<220 \\
<400\end{array}$ & $\begin{array}{l}26 \\
31\end{array}$ & ${ }_{10}^{8.7}$ & $\begin{array}{l}4.6 \\
5.2\end{array}$ & $\begin{array}{l}5.8 \\
6.0\end{array}$ & $\cdots$ \\
\hline
\end{tabular}

1' As shown on figure 1 . 


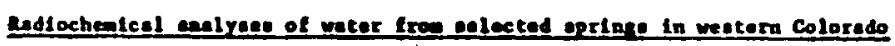

\begin{tabular}{|c|c|c|c|c|c|c|c|c|c|c|c|c|c|c|c|c|c|c|c|c|c|}
\hline \multirow[b]{2}{*}{ Owaer or tenant } & \multirow[b]{2}{*}{$\begin{array}{c}\text { mopple } \\
\text { point } \\
\text { number }\end{array}$} & \multicolumn{4}{|c|}{ location } & \multirow{2}{*}{\multicolumn{3}{|c|}{\begin{tabular}{|l|l|l|} 
Laticud \\
& 8 & \\
8 & & 8 \\
\end{tabular}}} & \multirow{2}{*}{$\begin{array}{r}\text { Lons } \\
8 \\
8\end{array}$} & \multirow{2}{*}{\multicolumn{2}{|c|}{$\begin{array}{l}\text { tude } \\
815 \\
8 \\
\end{array}$}} & \multicolumn{2}{|c|}{ 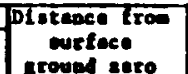 } & \multirow[b]{2}{*}{$\begin{array}{c}\text { Date } \\
\text { of } \\
\text { collection }\end{array}$} & \multicolumn{2}{|c|}{ mitiu } & \multicolumn{2}{|c|}{ Grove aph } & \multicolumn{2}{|c|}{ Groun bete } & \multirow[b]{2}{*}{ Lemerks: } \\
\hline & & 8 & 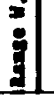 & 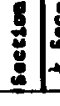 & 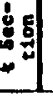 & & & & & & & $\begin{array}{l}\text { curf } \\
\text { scound } \\
\text { atles }\end{array}$ & 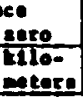 & & $\mathrm{pc1} / 2$ & Tu & 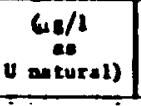 & $\begin{array}{c}(\mathrm{ccl} / \mathrm{l} \\
\mathrm{es} \\
\mathrm{U} \text { antural) }\end{array}$ & $\begin{array}{l}\operatorname{cc} 1 / 1 \\
00 \\
8 \mathrm{x}-90 \\
\mathrm{I}-90) \\
\end{array}$ & $\begin{array}{c}\text { (oct } / 1 \\
00 \\
c 0-137)\end{array}$ & \\
\hline Mro. Betty Potcer & 20 & 6 & 94 & 31 & $\mathbf{w}$ & 39 & 29 & 20 & 107 & 56 & 12 & 5.7 & 9.2 & $5-30-70$ & $<60$ & $\infty \infty$ & 26 & 1.8 & 9.2 & 9.8 & - \\
\hline Car1 Lernklau & 21 & 1 & 94 & 4 & $m$ & 39 & 28 & 09 & 107 & 53 & 43 & 5.1 & 6.2 & $5-30-70$ & $<60$ & $\infty 00$ & $<6.3$ & 8.1 & 3.2 & 3.8 & -- \\
\hline Town of Grend Valley & 22 & 1 & 95 & 5 & SE & 39 & 21 & 49 & 100 & $\infty$ & si & 3.3 & 8.5 & $5-30-30$ & $<60$ & $\infty 00$ & 1.9 & 2.6 & 3.1 & 3.4 & $\begin{array}{l}\text { row of Graod Velley } \\
\text { weter auply. }\end{array}$ \\
\hline OLL. Murrey & 23 & 7 & 96 & 3s & se & 39 & 23 & 23 & 108 & $\alpha$ & 20. & 6.0 & 11 & $5-30-70$ & 860 & $\infty 00$ & 11 & 3.6 & 5.5 & 6.1 & -- \\
\hline Cactl Gardner & 24 & 7 & 95 & 18 & $\mathbf{m}$ & 39 & 26 & 16 & 100 & 02 & 40 & 5.6 & 9.0 & $5-30-70$ & $\$ 60$ & $\infty 00$ & 42 & 14 & 3.0 & 5.6 & -- \\
\hline Fred Wallace & 25 & - & 95 & 24 & $\mathbf{N E}$ & 39 & $\mathbf{2 1}$ & of & 101 & 56 & 26 & 3.0 & 6.1 & $3-29-10$ & Q60 & $\infty$ & $<6$ & $<.2$ & 2.1 & 2.7 & $\cdots$ \\
\hline
\end{tabular}

(Voegeli and Claassen, 1971b) 
endlochemlcal emelyoes of water from ealected welle in weitern Colorado

\begin{tabular}{|c|c|c|c|c|c|c|c|c|c|c|c|c|c|c|c|c|c|c|c|}
\hline \multirow[b]{2}{*}{ inmer or tenant } & \multirow[b]{2}{*}{$\begin{array}{c}\text { Sample } \\
\text { point } \\
\text { numberl/ }\end{array}$} & \multicolumn{2}{|c|}{ Location } & \multicolumn{3}{|c|}{ Letitude N. } & N. 2 & \multicolumn{3}{|c|}{ Longl tude $H$. } & \multirow[b]{2}{*}{$\begin{array}{l}\text { Dietance froon } \\
\text { ourface ground } \\
\text { zero, In al lea } \\
\text { (kiliometers) }\end{array}$} & \multirow[b]{2}{*}{\begin{tabular}{c|} 
Date \\
of \\
collection
\end{tabular}} & \multicolumn{2}{|c|}{ Trittum } & \multicolumn{2}{|c|}{ Gross alpha } & \multicolumn{2}{|c|}{ Grose beta } & \multirow[b]{2}{*}{ Rewarke } \\
\hline & & ن & 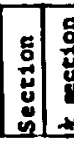 & 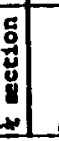 & $\begin{array}{ll}8 \\
8 \\
8\end{array}$ & 递 & $\begin{array}{l}: \\
: \\
8 \\
8 \\
\end{array}$ & $\begin{array}{l}: \\
8 \\
8 \\
\end{array}$ & 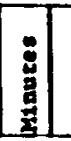 & $\begin{array}{l} \\
8 \\
8 \\
8\end{array}$ & & & $\mathrm{pci} / 1$ & $\mathrm{Tu}$ & $\begin{array}{c}(\mu 8 / 1 \\
a z \\
u \text { natura } 1)\end{array}$ & $\begin{array}{c}\text { (pci/1 } \\
\text { at } \\
0 \text { naturel) }\end{array}$ & $\begin{array}{c}(p c 1 / 1 \\
0 \vdots \\
5 x-90) \\
y-90) \\
\end{array}$ & {$\left[\begin{array}{c}(p c 1 / 1 \\
e \\
c a-137)\end{array}\right.$} & \\
\hline ..ur man Head & 15 & 694 & $26 \mathrm{~m}$ & N & 39 & 29 & 50 & 107 & 51 & 44 & $7.7(12)$ & $\begin{array}{l}3-24-69 \\
4-10-70\end{array}$ & $\begin{array}{l}1,300 \\
1,000\end{array}$ & $\begin{array}{l}420 \\
310\end{array}$ & $17^{6.8}$ & $\begin{array}{l}2.3 \\
5.6\end{array}$ & $\begin{array}{l}6.3 \\
7.0\end{array}$ & $\begin{array}{l}7.8 \\
7.3\end{array}$ & -- \\
\hline assell Bingham, $5 r$. & 16 & 794 & 65 & E & 39 & 274 & 41 & 1075 & 55 & 12 & $4.1(6.6)$ & $\begin{array}{r}3-22-69 \\
10-20-69 \\
4-10-70\end{array}$ & $\begin{array}{r}<700 \\
<960 \\
<1,300\end{array}$ & $\begin{array}{l}<220 \\
<300 \\
<400\end{array}$ & $\begin{array}{r}5.0 \\
<4.6 \\
4.8\end{array}$ & $\begin{array}{r}1.7 \\
<1.5 \\
1.6\end{array}$ & $\begin{array}{r}<3.5 \\
2.1 \\
3.9\end{array}$ & $\begin{array}{l}<4.3 \\
2.7 \\
4.3\end{array}$ & $\cdots$ \\
\hline Wert Gerdner & 17 & 795 & $20 \mathrm{M}$ & $w$ & 39 & 25 & 49 & 108 & 01 & 37 & $4.6(7.4)$ & $\begin{array}{l}3-26-69 \\
4-11-70\end{array}$ & $\begin{array}{r}960 \\
<1,300\end{array}$ & $\begin{array}{r}300 \\
<400\end{array}$ & $17^{9.1}$ & $\begin{array}{l}3.0 \\
3.6\end{array}$ & $\begin{array}{l}<.4 \\
4.1\end{array}$ & $\begin{array}{r}.5 \\
4.4\end{array}$ & -- \\
\hline inclatr ofl co. & 18 & 696 & $29 \mathrm{~s}$ & E & 39 & 29 & 31 & 108 & 07 & 23 & $11.1(17.9)$ & $\begin{array}{l}3-20-69 \\
4-12-70\end{array}$ & $\begin{array}{r}<700 \\
<1,300\end{array}$ & $\begin{array}{l}<220 \\
<400\end{array}$ & $\begin{array}{l}31 \\
26\end{array}$ & ${ }^{10} 8.6$ & $\begin{array}{l}15 \\
15\end{array}$ & $\begin{array}{l}19 \\
18\end{array}$ & -- \\
\hline illard Nicoll & 19 & 994 & $22 \mathrm{~N}$ & NE 3 & 391 & 15 & 49 & 107 & 52 & 02 & $10.6(17.1)$ & $\begin{array}{l}3-25-69 \\
4-11-70\end{array}$ & $\begin{array}{r}<700 \\
<1,300\end{array}$ & $\begin{array}{l}<220 \\
<400\end{array}$ & $\begin{array}{l}14 \\
34\end{array}$ & 11.8 & $\begin{array}{l}15 \\
6.0\end{array}$ & ${ }^{19} 7.3$ & -- \\
\hline
\end{tabular}




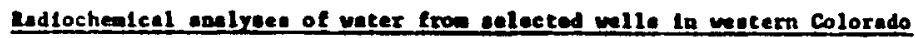

\begin{tabular}{|c|c|c|c|c|c|c|c|c|c|c|c|c|c|c|c|c|c|c|c|c|c|}
\hline \multirow[b]{2}{*}{ Omar or tenant } & \multirow[b]{2}{*}{$\begin{array}{c}\text { Sample } \\
\text { polste } \\
\text { numberd }\end{array}$} & \multicolumn{4}{|c|}{ Locetion } & \multicolumn{3}{|c|}{ Letioud } & \multicolumn{3}{|c|}{ Lonifrude th } & \multirow{2}{*}{\multicolumn{2}{|c|}{$\begin{array}{l}\text { pl stmence fron } \\
\text { murfece } \\
\text { mound gero }\end{array}$}} & \multirow[b]{2}{*}{$\begin{array}{c}\text { Date } \\
\text { of } \\
\text { colleetion }\end{array}$} & \multicolumn{2}{|c|}{ mitie } & \multicolumn{2}{|c|}{ Groes alphe } & \multicolumn{2}{|c|}{ Gropl bet } & \multirow[b]{2}{*}{ Remerke } \\
\hline & & 8 & \begin{tabular}{|l|}
$x$ \\
0 \\
0 \\
\end{tabular} & 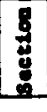 & $\begin{array}{r}8 \\
0 \\
-5 \\
\end{array}$ & $\begin{array}{l}8 \\
8 \\
8 \\
\end{array}$ & $\begin{array}{l}3 \\
8 \\
2\end{array}$ & 8 & 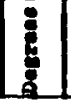 & 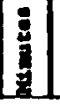 & 8 & & 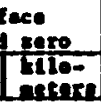 & & $p c 1 / 1$ & Tv & $\begin{array}{c}\text { (us/1 } \\
0 \\
0 \text { matural) }\end{array}$ & $\begin{array}{c}\text { (fci/1 } \\
\text { eat } \\
\text { neturel })\end{array}$ & 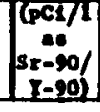 & $\begin{array}{c}(6 c t / 1 \\
0 \\
c a-13 n)\end{array}$ & \\
\hline Norman Mend & 15 & 6 & 94 & 26 & $\mathbf{m}$ & 39 & 29 & so & 107 & 51 & 44 & 7.7 & 12 & $5-30-70$ & 860 & 800 & $<.1$ & 0.0 & 9.3 & $\mathbf{n}$ & - \\
\hline Zuesell Blaghes, Sx. & 16 & 7 & 94 & 6 & st & 39 & 21 & 41 & 101 & ss & 12 & 4.1 & 6.6 & $5-30-70$ & $\infty 6$ & $\infty \infty$ & 9.0 & 3.3 & 4.1 & 4.4 & -- \\
\hline Albert Gardner & 17 & 7 & 95 & 20 & 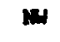 & 39 & 25 & 49 & 108 & 01 & 31 & 4.6 & 1.4 & $5-30-70$ & 060 & 800 & 13 & 4.3 & 3.7 & 6.4 & - \\
\hline S1aclatr ofl co. & 18 & 6 & 96 & 29 & sE & 39 & 29 & 31 & 108 & 01 & 23 & $\mathbf{2 1 . 2}$ & 17.9 & $5-30-70$ & $Q 60$ & $\infty 00$ & 27 & 9.0 & 23 & 26 & -- \\
\hline
\end{tabular}

(Voegeli and Claassen, 1971b) 


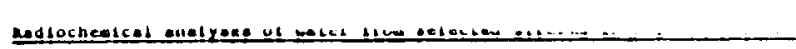

\begin{tabular}{|c|c|c|c|c|c|c|c|c|c|c|c|c|c|c|c|c|c|c|c|c|c|c|c|c|}
\hline \multirow[b]{3}{*}{ strean } & \multirow{3}{*}{$\begin{array}{c}\text { sample } \\
\text { point } \\
\text { numbert }\end{array}$} & \multicolumn{2}{|c|}{ Eocotion } & \multicolumn{6}{|c|}{ 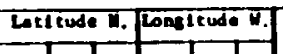 } & & & \multirow{3}{*}{$\begin{array}{c}\text { Dote } \\
0 ! \\
\text { collect }\end{array}$} & \multicolumn{2}{|c|}{ mitium } & \multicolumn{4}{|c|}{ Dienolved } & \multicolumn{5}{|c|}{ Busposided } & \multirow[b]{3}{*}{ Restrke } \\
\hline & & \multirow{2}{*}{ : } & \multirow{2}{*}{\begin{tabular}{|l|l} 
\\
\end{tabular}} & \multirow[b]{2}{*}{ 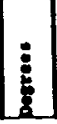 } & \multirow[b]{2}{*}{ : } & & \multirow[b]{2}{*}{8} & \multirow[b]{2}{*}{ 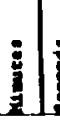 } & & & & & \multirow{2}{*}{$\mathrm{pcs} / 1$} & \multirow[b]{2}{*}{$\pi$} & \multicolumn{2}{|c|}{ Grose- -1pee } & \multirow{2}{*}{ 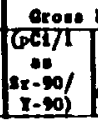 } & \multirow{2}{*}{$\mid \begin{array}{c}\text { sect } / 1 \\
00 \\
c e-137\end{array}$} & \multirow{2}{*}{ sollde } & \multicolumn{2}{|c|}{ Orope plpa } & \multirow{2}{*}{ 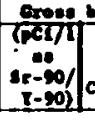 } & \multirow{2}{*}{ 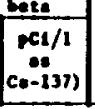 } & \\
\hline & & & & & & & & & हु & & & & & & me/la as & 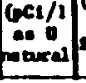 & & & & o cus/t as as & 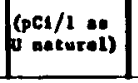 & & & \\
\hline $\begin{array}{l}\text { Roartias Fork } \\
\text { River aest } \\
\text { Aupeos }\end{array}$ & 1 & & $(2 / 3$ & 39 & 10 & 40 & 1064 & 48 & os 6 & 64 & 103 & $\begin{array}{l}6-1-70 \\
6-23-70\end{array}$ & 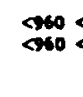 & 800 & $\begin{array}{l}2.7 \\
2.2\end{array}$ & 0.9 & 3.5 & 3.5 & ?0 & 4.1 & 1.4 & $\begin{array}{l}3.3 \\
1.0\end{array}$ & $\begin{array}{l}3.8 \\
1.1\end{array}$ & $\begin{array}{l}\text { Uses gagine otation } \\
9-0 i 3 .\end{array}$ \\
\hline $\begin{array}{l}\text { colorado aiver } \\
\text { tr keu Castle }\end{array}$ & 2 & 59 & $9031 \mathrm{su}$ & 39 & 34 & 06 & 101 & 32 & 262 & 25 & 40 & $\begin{array}{l}6-1-70 \\
6-25-70\end{array}$ & $\begin{array}{l}<960 \\
1,100\end{array}$ & $\begin{array}{l}000 \\
360\end{array}$ & 3.3 & $\begin{array}{l}1.2 \\
1.5\end{array}$ & 6.3 & 3.4 & 100 & 3.7 & 1.9 & 3.8 & 5.6 & $\begin{array}{l}\text { uscs gegtand ocacton } \\
\text { T-0676. }\end{array}$ \\
\hline $\begin{array}{l}\text { Buver Creek } \\
\text { near nille }\end{array}$ & 3 & 19 & $941 \mathrm{NE}$ & 39 & 20 & 20 & 107 & 49 & ss & 1.6 & 12 & $\begin{array}{l}3-30-10 \\
6-25-10\end{array}$ & $\begin{array}{l}9000 \\
1,000\end{array}$ & $\begin{array}{l}800 \\
320\end{array}$ & 1.1 & .4 & $\begin{array}{l}3.9 \\
2.1\end{array}$ & 3.5 & $\begin{array}{r}210 \\
32\end{array}$ & 12. & .0 & i.: & 2.1 & $\begin{array}{l}\text { uses gagtine otation } \\
\text { g-0925. }\end{array}$ \\
\hline $\begin{array}{l}\text { Kinball Creetk } \\
\text { nest Collbran }\end{array}$ & 4 & 99 & $9314 \mathrm{kR}$ & 39 & 11 & $\infty$ & 107 & 51 & 13 & 8.4 & 14 & $\begin{array}{l}3-29-10 \\
6-29-70\end{array}$ & $\begin{array}{l}<960 \\
1.200\end{array}$ & 000 & 2.1 & .7 & $\begin{array}{l}2.1 \\
2.1\end{array}$ & 3.6 & $\begin{array}{c}160 \\
34\end{array}$ & $\because: 3$ & 3.3 & 3.4 & 6.5 & $\cdots$ \\
\hline $\begin{array}{l}\text { Placesu Craek } \\
\text { near Canco }\end{array}$ & s & 109 & $9718 \mathrm{sW}$ & 39 . & 11 & $\infty$ & 108 & 16 & 102 & 23 & 37 & $\begin{array}{l}5-29-70 \\
6-25-70\end{array}$ & $\begin{array}{l}<960 \\
<>60\end{array}$ & 000 & 12.9 & 1.3 & $11^{3.3}$ & $16^{6.3}$ & $\begin{array}{r}330 \\
40\end{array}$ & 27.6 & .9 & 3.4 & is: & $\begin{array}{l}\text { usses gagises atation } \\
\text { g-1050. }\end{array}$ \\
\hline $\begin{array}{l}\text { Coloroda Miver } \\
\text { near Deleque }\end{array}$ & 6 & 89 & 97 $23 \mathrm{sw}$ & 39 & 20 & 22 & 108 & 11 & $3 s$ & 14 & 23 & $\begin{array}{l}5-29-70 \\
6-25-70\end{array}$ & $\begin{array}{l}<960 \\
<960\end{array}$ & $\begin{array}{l}000 \\
000\end{array}$ & 3.1 & 1.6 & $\because .8$ & 13.5 & 180 & 3.6 & i.? & 3.8 & 10.7 & 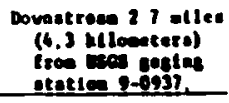 \\
\hline
\end{tabular}

(Voegeli and Claassen, 1971b) 


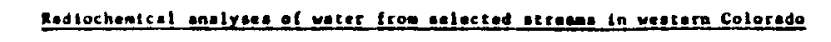

\begin{tabular}{|c|c|c|c|c|c|c|c|c|c|c|c|c|c|c|c|c|c|c|c|c|c|c|c|}
\hline \multirow{3}{*}{ Strean } & \multirow{3}{*}{$\left|\begin{array}{c}\text { seaple } \\
\text { poins } \\
\text { numbers }\end{array}\right|$} & \multicolumn{2}{|l|}{ Locsition } & \multicolumn{5}{|c|}{ Lit tude $\mathrm{M}$. Longl tude $\mathrm{H}$. } & \multirow{2}{*}{\multicolumn{2}{|c|}{ 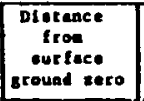 }} & \multirow{3}{*}{$\begin{array}{c}\text { Date } \\
\text { of } \\
\text { collection }\end{array}$} & \multicolumn{2}{|c|}{ Iriction } & \multicolumn{4}{|c|}{ Diocolved } & \multicolumn{5}{|c|}{ Sulpopoded } & \multirow{3}{*}{ Deenrke } \\
\hline & & $\therefore 1.1$ & 5 & & & & & & & & & \multirow[b]{2}{*}{$p C_{1} / 1$} & \multirow[b]{2}{*}{$\pi$} & \multicolumn{2}{|c|}{ Groses elphe } & \multicolumn{2}{|c|}{ Grome betes } & \multirow{2}{*}{$2011 d \theta$} & \multicolumn{2}{|c|}{ Grone alphe } & \multicolumn{2}{|c|}{ Crosi Dete } & \\
\hline & & 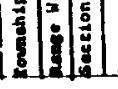 & 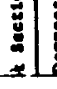 & & 8 & 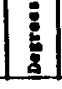 & $\begin{array}{r}\mathbf{z} \\
\vdots \\
z \\
z \\
\end{array}$ & & sround & nero- & & & & 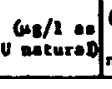 & {$\left[\begin{array}{c}(\mathrm{pci} / 1 \\
00 \\
n a t u r e\end{array}\right]$} & {$\left[\begin{array}{c}p c 1 / 1 \\
20 \\
i r-90 / \\
i-20\end{array}\right] c$} & {$\left[\begin{array}{c}6 \mathrm{pct} / 1 \\
\mathrm{es} \\
\mathrm{cos}-137\end{array}\right]$} & & $\begin{array}{c}\text { (us } / 1 \\
0 \text { anturat) }\end{array}$ & 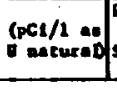 & 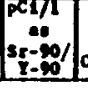 & $\begin{array}{c}c c 1 / 1 \\
00 \\
c 0-13 n)\end{array}$ & \\
\hline $\begin{array}{l}\text { Rouring York } \\
\text { River ness } \\
\text { Aspen }\end{array}$ & 1 & $12 n$ & & 39 IC & 1048 & 106 & 48 & 0) 64 & 64 & 103 & $\begin{array}{r}2-17-70 \\
9-30-70 \\
10-10-70\end{array}$ & $\begin{array}{l}.800 \\
1.000 \\
740\end{array}$ & $\begin{array}{l}240 \\
330 \\
230\end{array}$ & & & & & & & & & & 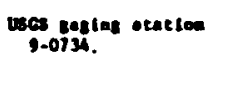 \\
\hline $\begin{array}{l}\text { Culorado River } \\
\text { "I Mou castle }\end{array}$ & 2 & os 9031 & sw & 393 & 3406 & 107 & 32 & 2025 & 25 & 40 & 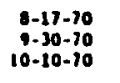 & $\begin{array}{l}i, 000 \\
i 1000\end{array}$ & $\begin{array}{r}320 \\
330 \\
2200\end{array}$ & & & & & & & & & & $\begin{array}{l}\text { UsGs geglins atetion } \\
\text { g-0ath. }\end{array}$ \\
\hline $\begin{array}{l}\text { Beaver Creck } \\
\text { gear Rifie }\end{array}$ & 3 & 089608 & NE & 392 & 2820 & 107 & 49 & ss 1 & 1.6 & 12 & 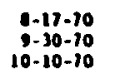 & $\begin{array}{l}800 \\
910 \\
<100\end{array}$ & $\begin{array}{l}\mathbf{2 2 0} \\
280 \\
220\end{array}$ & & & & & & & & & & 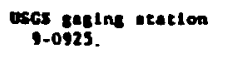 \\
\hline $\begin{array}{l}\text { Rimball Creek } \\
\text { nest colloreso }\end{array}$ & 4 & 099514 & $\mathrm{ME}$ & 391 & 1700 & 107 & s) & 13 & 0.4 & 14 & $\begin{array}{r}-11-10 \\
;-30-10 \\
10-11-10\end{array}$ & $\begin{array}{l}>100 \\
<>0 \\
<100\end{array}$ & $\begin{array}{l}220 \\
220 \\
920\end{array}$ & & & & & & & & & & $-\cdot$ \\
\hline $\begin{array}{l}\text { Placesu Creek } \\
\text { near cooseo }\end{array}$ & $s$ & 10918 & sw & 391 & 1100 & 108 & 16 & 1023 & 23 & " & $\begin{array}{c}0-11-20 \\
9-30-20 \\
10-11-70\end{array}$ & $\begin{array}{r}.990 \\
1.000 \\
110\end{array}$ & $\begin{array}{l}310 \\
320 \\
250\end{array}$ & $\begin{array}{l}13 \\
32\end{array}$ & 12.9 & 6.7 & $3^{11}$ & is & $e^{3.1}$ & $2 i^{1.0}$ & ${ }_{25}^{2.4}$ & $\underset{21}{3.0}$ & 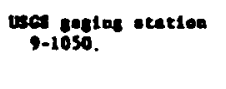 \\
\hline $\begin{array}{l}\text { Colurado River } \\
\text { near Diseque }\end{array}$ & 6 & oa 9123 & sw & 392 & 2022 & 108 & $n$ & 3514 & 14 & 23 & $\begin{array}{r}2-11-70 \\
9-30-70 \\
10-11-70\end{array}$ & $\begin{array}{l}1,000 \\
1,000 \\
000\end{array}$ & $\begin{array}{l}320 \\
320 \\
230\end{array}$ & $\begin{array}{l}3.1 \\
13\end{array}$ & 1:? & $\begin{array}{l}3.8 \\
8.2\end{array}$ & 3.3 & $\begin{array}{r}33 \\
100 \\
490\end{array}$ & 39.9 & $\underset{13}{<1}$ & $\begin{array}{c}2.0 \\
21.3 \\
20\end{array}$ & $\begin{array}{l}24.4 \\
26.4\end{array}$ & 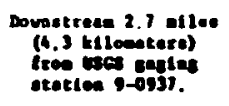 \\
\hline
\end{tabular}

$\checkmark$ as slown on tizure 1.

3/ Mot eurveyed.

(Claassen and Voegeli, 1971) 


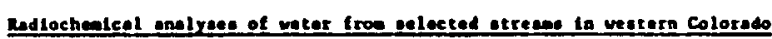

\begin{tabular}{|c|c|c|c|c|c|c|c|c|c|c|c|c|c|c|c|c|c|c|c|c|c|c|c|c|c|}
\hline \multirow[b]{3}{*}{ streas } & \multirow{3}{*}{ semple } & \multicolumn{3}{|c|}{ Locetion } & \multirow{2}{*}{\multicolumn{6}{|c|}{ Leticude Lenglude }} & \multirow{3}{*}{\multicolumn{2}{|c|}{ 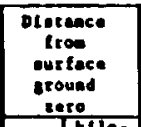 }} & \multirow{3}{*}{$\begin{array}{c}\text { Dose } \\
\text { ei } \\
\text { collection }\end{array}$} & \multirow{2}{*}{\multicolumn{2}{|c|}{ sritive }} & \multicolumn{4}{|c|}{ Deooling } & \multicolumn{5}{|c|}{ Sugereoded } & \multirow{3}{*}{ Iements } \\
\hline & & & & 8 & & & & & & & & & & & & \multicolumn{2}{|c|}{ Crose elphe } & \multicolumn{2}{|c|}{ Grose bates } & \multirow{2}{*}{ Sollde } & \multicolumn{2}{|c|}{ Groese slphese } & \multicolumn{2}{|c|}{ Grove bete } & \\
\hline & & : & 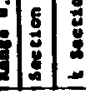 & 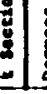 & 8 & : & 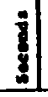 & 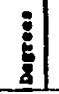 & 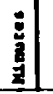 & 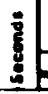 & & & & $\mathrm{pes} / \mathrm{l}$ & $\mathfrak{n}$ & \begin{tabular}{|c|}
$6 x / 2$ \\
018 \\
woturas \\
\end{tabular} & 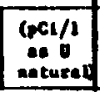 & $\mid \begin{array}{c}\text { C्CII } \\
00 \\
3 x+90 / \\
x-201\end{array}$ & {$\left[\begin{array}{c}(x+1 / 1 \\
00 \\
c=-13 n\end{array}\right]$} & & $\mid \begin{array}{c}4 a / 1 \\
0 \text { actrats }\end{array}$ & \begin{tabular}{|l|} 
(oct/1 as \\
0 asturat) \\
\end{tabular} & 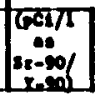 & $\begin{array}{l}6(1 / 1 \\
01 \\
c:-13 n)\end{array}$ & \\
\hline $\begin{array}{l}\text { Roortag Fork } \\
\text { Mivex acer } \\
\text { Aopeen }\end{array}$ & $\mathbf{1}$ & & $a h$ & & 39 & 20 & 48 & 106 & 48 & os & 64 & 103 & $\begin{array}{l}10-23-70 \\
11-4-70\end{array}$ & $\begin{array}{l}1,000 \\
990\end{array}$ & $\begin{array}{l}320 \\
310\end{array}$ & & & & & & & & & & 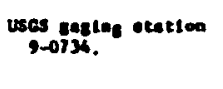 \\
\hline $\begin{array}{l}\text { Culoredo Rivar } \\
\text { ac Now Caatie }\end{array}$ & 2 & os & $9031 \mathrm{sm}$ & & 39 & 34 & ob & 101 & 32 & 26 & 23 & 40 & $\begin{array}{c}y_{11=8-30}^{10-23-70} \\
11=90\end{array}$ & $\begin{array}{r}1.000 \\
860\end{array}$ & $\begin{array}{l}330 \\
370\end{array}$ & -- & $\cdots$ & - & 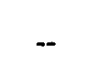 & -- & $*$ & $\cdots$ & $\cdots$ & -- & $\begin{array}{l}\text { uscs gegeling otation } \\
\text { q-obit. }\end{array}$ \\
\hline $\begin{array}{l}\text { Beaver Creek } \\
\text { near Rifle }\end{array}$ & 3 & 01 & 96 ol Mr & & 39 & 20 & 20 & 107 & 69 & ss & 1.6 & 12 & $\begin{array}{c}10-23-70 \\
11=3-70 \\
11-10\end{array}$ & $\ddot{1000}$ & $\frac{200}{020}$ & -• & $\cdots$ & $\cdots$ & $\cdots$ & -- & $\cdots$ & -- & -- & - & uses gegtase otest 100 \\
\hline $\begin{array}{l}\text { Kinball creak } \\
\text { near collbran }\end{array}$ & 4 & 09 & $9314 \mathrm{NI}$ & & 39 & 11 & $\infty$ & 10) & 37 & 13 & 8.4 & 14 & $\begin{array}{l}10-26-10 \\
211=9-10 \\
11=9-10\end{array}$ & 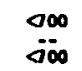 & $\begin{array}{l}920 \\
\ddot{820}\end{array}$ & -- & $-\cdot$ & -- & -- & -- & $\cdot-$ & -- & - & - & $\cdots$ \\
\hline $\begin{array}{l}\text { Pleceau Creek } \\
\text { neer Caaeeo }\end{array}$ & $s$ & 10 & $9718 \mathrm{st}$ & & 39 & $" 1$ & $\infty$ & 108 & 16 & 10 & 23 & 37 & $\begin{array}{l}10-24-10 \\
11-5-10\end{array}$ & 960 & $\begin{array}{l}290 \\
310\end{array}$ & 26 & 86 & ${ }_{23}^{10}$ & 32 & 31 & 1.2 & 0.4 & 4.2 & i: & 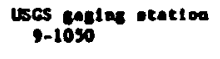 \\
\hline $\begin{array}{l}\text { Coloredo River } \\
\text { near De leque }\end{array}$ & 6 & 08 & $9723 \mathrm{st}$ & & 39 & 20 & 22 & 100 & 11 & $3 s$ & 14 & 23 & $\begin{array}{r}10-23-10 \\
=11-9-10 \\
11-9-10\end{array}$ & $\begin{array}{l}900 \\
900\end{array}$ & $\begin{array}{l}280 \\
\ddot{280}\end{array}$ & $\begin{array}{l}24 \\
\text { ii }\end{array}$ & $\begin{array}{l}.0 \\
3.5\end{array}$ & $\underset{62}{6.6}$ & $\because$ & $\because 6$ & $\begin{array}{l}2.6 \\
1.2\end{array}$ & .9 & $\begin{array}{l}4.3 \\
2.3\end{array}$ & $\begin{array}{l}.46 \\
2.7\end{array}$ & 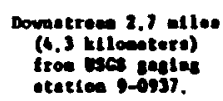 \\
\hline
\end{tabular}

$\checkmark$ S. shown on figure 1 .

d mot surveyed.

a samples for tritium, aroes alpha, end gross bete collectod ond etored.

(Claassen, 1971, p. 4) 


\section{Distribution List}

\section{$\underline{\text { Copies }}$}

U.S. Department of Energy

Nevada Operations Office

Technical Information Resource Center

P.O. Box 98518

Las Vegas, NV 89193-8518

U.S. Department of Energy

Office of Scientific and Technical Information

175 Oak Ridge Turnpike

Post Office Box 62

Oak Ridge, Tennessee 37831

U.S. Department of Energy

Public Reading Room

P.O. Box 98521, M/S NLV040

Las Vegas, NV 89193-8521

Donna Stoner

Colorado Department of Public Health \& Environment

Hazardous Materials \& Waste Management Division

722 South $6^{\text {th }}$ Street, Room 232

Grand Junction, Colorado 81501

Parachute Branch Library

1

Rulison Public Reading Room

244 Grand Valley Way

Parachute, Colorado 81635

Central Files

IT Corporation

2621 Losee Rd., Bldg. B-1, 3050-01

North Las Vegas, Nevada 89030 